\title{
Wave modelling in coastal and inner seas
}

3 authors

4 L.Cavaleri $^{1 *}$, S.Abdalla ${ }^{2}$, A.Benetazzo ${ }^{1}$, L.Bertotti ${ }^{1}$, J.-R.Bidlot ${ }^{2}$, Ø.Breivik ${ }^{3}$, S.Carniel $^{1}$,

5 R.E.Jensen ${ }^{4}$, J.Portilla ${ }^{5}$, W.E.Rogers ${ }^{6}$, A.Roland ${ }^{7}$, A.Sanchez-Arcilla ${ }^{8}$, J.M.Smith ${ }^{4}$, J.Staneva ${ }^{9}$,

6 G.P.van Vledder ${ }^{10}$, A.J. van der Westhuijsen ${ }^{11}$

9 Affilitions:

101 - Istituto di Scienze Marine - CNR, Venice, Italy

112 - European Centre for Medium-Range Weather Forecasts, Reading, U.K.

123 - Norwegian Meteorologisk Instituut, Bergen, Norway

134 - US Army Engineer Research and Development Center, Vicksburg, MS, USA

145 - Escuela Politecnica National, Quito, Ecuador

156 - Naval Research laboratory, Stennis Space Center, MS, USA

167 - BGS IT\&E GmbH, Darmstadt, Germany

178 - Universitat Politecnica de Catalunya, Barcelona, Spain

189 -Helmholtz-Zentrum Geesthacht, Germany

1910 - Delft University of Technology, Delft, The Netherlands

2011 - NWS/NCEP/Environmental Modeling Center, College Park, MD, USA

*) reference author: luigi.cavaleri@ ismar.cnr.it 
36 In the long term development of the research on wind waves and their modelling, the present 37 situation is framed with a short look at the past, a critical analysis of the present capabilities and a 38 foresight of where the field is likely to go. After a short introduction, Chapter 2 deals with the basic 39 processes at work and their modelling aspects. Chapter 3 highlights the interaction with wind and 40 currents. Chapter 4 stresses the need for a more complete, spectral, approach in data assimilation. 41 Chapter 5 summarizes the situation with a discussion on the present status in wave modelling and a 42 look at what we can expect in the future. 
1 - Wave challenges in coastal and inner seas

45 a review of the present know-how, results, problems and expectations in this not large environment, but with a lot of connections to it

It is amply acknowledged that wave modelling has now achieved a high degree of reliability. Global modelling of the best operational centres regularly provide analyses and forecasts with an accuracy

(see, among

others,

51 http://www.ecmwf.int/en/forecasts/charts/obstat/?facets=Category,Satellite\%20Data\%3BParameter,

52 Surface\%20wind\%20speed (wind speed) and

53 http://www.ecmwf.int/en/forecasts/charts/obstat/?facets=Parameter,Wave\%20Height (wave height)

54 for the European Centre for Medium-Range Weather Forecasts (ECMWF, Reading, U.K.) and 55 http://polar.ncep.noaa.gov/nwps/ for the National Center for Environmental Prediction (NCEP, 56 Maryland, USA). Also in the middle range, forecasts are generally (but not always) reliable till one week in advance. This is due to the substantial improvements progressively achieved in meteorological modelling and, particularly in the middle range, to recent refinements in the physics of generation and dissipation of wind waves. Problems still exist and require attention, particularly in view of the growing acknowledgement of the role of wind waves in modulating all the exchanges at the air-sea interface, and therefore having a basic role in determining the Earth climate. However, from the point of view of traditional wave applications, the general user can be quite satisfied.

This is not always the case in restricted (coastal and semi-enclosed) seas. The obvious affecting factors are the presence of land and associated orography, and, on purely marine terms, the presence of often extended areas of shallow waters. Land and orography substantially affect the wind fields, with immediate consequences on the evolution of the local wave fields. The presence of shallow waters, with different kinds of bottom, either rocky or sandy, and possibly covered with vegetation, mud or, in the Arctic Ocean, ice, complicates or changes which are the dominant processes at work, hence the relevance of the accuracy of the background information. On very shallow water (kh < 0.5) the details of the bottom effects may become the dominant factors, especially with small grids with a high spatial resolution. On the application side, in limited depth areas the wave conditions may become the relevant information for, e.g., the local biological conditions, sea productivity and the corresponding proper management. On the upper side of the limited basins the so-called Arctic ocean should be included as well, its present dimensions when free of ice being comparable to some of the enclosed areas we regularly deal with, e.g., the Great Lakes of North America. The extra factor to be considered is obviously the waves-ice interactions. 
79 An often emerging difference with respect to deep open waters is the relevance of currents. In the oceans, with the exception of well determined areas, most of the time and in most of the places the surface currents do not reach velocities such to substantially affect wave conditions (for the time being we purposely ignore the wave induced currents). Therefore also the frequent lack of accuracy in the details that characterizes most of the large scale circulation models is not likely to appreciably affect the local wave results, at least for waves of a certain dimensions, hence of general interest for most of the users. This is not the case close to the coast. Here quite often the currents (barotropic and baroclinic) are geographically enhanced reaching values that, if not considered, can lead to substantial errors in wave model results. This is more frequently the case in semi-enclosed seas, where the limited dimensions imply in general shorter wave periods than in the oceans, hence more sensitive to the influence of currents.

91 The interactions between waves and current act in both the directions, sometimes with a positive 92 feed-back effect, forcing on one hand to consider these interactions in their various facets, and on 93 the other hand to pay much more attention to current modelling to achieve, as far as possible, the accuracy required for the one desired for wave model results. It is rather intuitive that, given the smaller time and spatial scales in the inner seas, the relevance of "smaller" (high resolution) details is high, and it implies a shortening of the reliable range of forecast. There are two reasons for this.

97 On very general terms the smaller is an important detail, the more likely a forecast is to be affected 98 by errors, because of the inaccuracies of the initial conditions and the imperfections of the model. 99 More specifically for the coastal areas and semi-enclosed seas, the local conditions are much more 100 sensitive than in the open sea to, e.g., a slight shift of the forcing meteorological pattern, either in 101 space or time, with respect to the local geometry. When looking at the coastal meteorological surge, 102 the phasing relative to astronomical tide becomes crucial, a simple time shift, of, e.g., three hours of 103 the meteorological event possibly leading to completely different overall conditions on the coast.

104 This implies a shortening of the useful range of forecast because the error is growing with range 105 faster than in the open sea.

On the other hand, there is a steady growth of the already intense interest in the wave conditions in coastal areas, both at local and a more extended scale. Increasing maritime traffic, recreational activities, urban development, ecosystem restoration, renewable energy industry, offshore management, all push in this direction. The purpose of this paper is to frame the present situation in wave modelling in coastal waters and in the enclosed seas. We do not aim at a review of the 
112 existing literature (a daunting task), but rather to touch the main subjects of relevance in coastal and

113 semi-enclosed sea wave modelling, citing sufficient examples of the relevant literature. The 114 emphasis will be on the problems that still affect this topic. We stress the physics involved, and in 115 turn this will imply to touch, but not to dig in, the field of meteorological and circulation modeling 116 because of the tight coupling in a spatially limited environment. All this will be complemented with 117 an extensive range of applications, both to frame the possible accuracy and to call the attention, via 118 the contemporary use of different models for the same event, to the differences and difficulties we 119 still find in practical applications.

121 Based on this approach the paper is structured as follows.

123 In Chapter 2 we deal with the basic processes at work, analysing the various modelling aspects that 124 lead to, and condition, the final results.

125 In particular Section 2.1 analyses the reasons why the wind model input information are 126 likely to be less correct than in the open ocean. We also stress the higher variability and that very high wave conditions are possible also in enclosed seas.

Section 2.2 deals with the basic aspects of wave modelling in this relatively restrained environment. It explains the reasons of the greater difficulty to obtain good results with respect to 130 the open sea.

Section 2.3 focuses more on this aspect, detailing the physics involved.

In Section 2.4 we leave physics (partially) aside to discuss the crucial aspect of any numerical model, its numerics, i.e. how the various equations are integrated in space and time. Although the models are (partially) built with some self-control mechanism, we stress that every user should be aware of the approximations involved, and of the consequent likely accuracy of the final results.

Section 2.5 focuses on a crucial aspect of the validation of our model results, i.e. the accuracy of the measured data we use to compare with. While we touch most of the main instruments at use in the world, we devote quite a bit of attention to buoys. These have been for decades the almost official reference for the calibration of other instruments, especially from satellites. For this reason we devote quite a bit of space to this analysis, just to make the unaware wave modeller aware of the implied approximations.

144 different environments to highlight the various problems we (may) face in practical use and the 145 accuracy we can expect in the various conditions. 
147 In Chapter 3 we abandon the view of modelling waves as an isolated process, and we deal with the interaction with the two media waves involve when moving.

Section 3.1 deals with the interaction with sea currents, and how wave and currents interact with mutual and feed-back effects. We show this in a number of examples in quite different environments.

In Section 3.2 we extend this mutual interaction also to the atmosphere. This interaction can

153 be particularly intense in coastal waters due to the enhanced effects associated to orography and 154 limited coastal depths. The extensive citation of the existing literature is a clear proof of the complexity of these three-component interactions, exemplified in a number of cases.

Chapter 4 discusses data assimilation in enclosed seas. While the described principles are quite general, we highlight the related problems in this specific environment. In particular we stress that long term used approaches, as e.g. Optimal Interpolation, are generally not suitable for the constrained geometry of the enclosed seas. Therefore we focus our attention on a spectral approach that two examples show to be more suitable, especially for a complicated geometry.

In Chapter 5 we make an extensive summary of the situation. We discuss the quality of the present approaches, the reliability of the results, and what we must be aware of when modelling waves in enclosed seas. We also make an outlook into the future discussing the expected or likely developments, which problems are technical, hence with a foreseeable development, and which are physical, when knowledge and theory are not necessarily moving at a regular pace.

The bibliography is quite comprehensive, each Chapter and Section requiring its own share of know-how and historical and modern developments. The Appendix lists the most common and repetitively used acronyms with their meaning.

173 Being the product of multiple contributions, there is not a unique style of writing. While an effort of 174 homogenization has been done, unavoidably each master hand leaves a different trace. More importantly, the development of the paper follows a logical flow, in a way from relatively simple to more complicated matters. So a full reading makes sense, but it is not strictly necessary. Each 
179 As expected, and being the product of a community of wave modellers, there is an ample list of 180 authors. Different specializations and contributions are reflected into the authors of each Section. 181 Some more names may be listed here meaning some specific limited contributions. Questions, 182 discussion and requests can be addressed to the first author of each Section (e-mail address 183 provided).

184

185 


\section{2 - Wave modelling}

where we make a panorama of the present situation in coastal and enclosed seas, starting from meteorology, physics and numerics, ending with a keen discussion on the data we use for validation. Specific examples of application help to focus the problems we still face today

Nowadays we are used to rely heavily on weather forecast and the derived quantities. Wind, temperature, and possibly rain, are the main relevant information of interest on land. On the contrary, granted the relevance of the overall situation, waves are the crucial factor conditioning all our sea activities, often till a dangerous level. It is therefore not surprising that wave analysis and forecast have received so much attention since the second world war. Starting with the physical approach by Miles (1957) and Phillips (1957), and following the basic concept of energy balance equation (Gelci et al., 1957), the last sixty or so years have witnessed a continuous intense improvement. Cavaleri et al. (2007) provided a thorough picture of the situation, also hinting at the expected further developments in the near future. As pointed out in this cited paper, there have been moments of drastic advancement followed by periods of consolidation, the new findings being quickly implemented in the operational and research models. Unavoidably the rate of improvement has decreased in time while our models are becoming better and better, slowly approaching the sort of ideal we can have in our mind (but there are almost certainly still problems to be discovered as we become more and more demanding in our search for perfection). On the whole we can be quite pleased with the results. See the statistics of, among others, ECMWF and NOAA/NCEP cited in the previous section.

In the inner seas, judging from the quality of the results, we seem to be late in this rate for improvement. Errors are more frequent as wave height, period and direction, timing is more approximate. There are two basic reasons for this. On one hand the meteorological input, the frequent culprit invoked by wave modellers, is less accurate than in the open oceans. The geometry of the coasts and the frequently complicated orography take their toll. In a limited fetch, in coastal areas, in general in an enclosed sea, a small difference of the wind direction can lead to drastically different wave results. Still on the geometrical side, small islands or shallow zones become important, but they are frequently not correspondingly represented, with their characteristics, in the wave model. On the other hand, while, granted the storm belts, the open ocean is characterized mainly by swell, the inner seas are dominated by wind sea. In a general sense, swell is an established situation, and most of the times the related essential operation in wave modelling is advection. Ardhuin et al. (2010) pointed out the swell possible attenuation over long distances mainly due to the interaction with the adjacent atmospheric surface boundary layer. However, we 
can call this a second order effect, probably also less relevant in the inner seas where the distances are reduced with respect to the open space of the oceans. Inner basins are characterized mainly by

224 wind sea, that in a way can be considered as a transient situation, or at least something whose dynamical state is established by the dynamical balance of all the main "forces" (input by wind, breaking and dissipation, non-linear interactions) acting on the field. This is much more difficult for a correct quantification in space and time.

In this section we discuss the various aspects affecting the performance of our wave models in enclosed seas. After analysing (2.1) the role of meteorology and the related problems, especially in coastal areas, we focus first on deep water (2.2) quantifying the present accuracy in the inner seas areas compared to the oceans. Then we move to shallow water (2.3) pointing out the additional processes that in this environment affect the energy balance of the wave systems. In (2.4) we go into the details of wave modelling in these specific conditions, discussing the various approaches and the related problems and accuracy. Data availability and the associated accuracy and reliability are analysed in (2.5). Finally, in (2.6) we present practical applications as examples of the various mentioned aspects relevant for the accuracy of the final results.

\section{R.E.Jensen and L.Cavaleri}

\section{Robert.E.Jensen@erdc.dren.mil}

\section{1 - The meteorological factor}

where we describe the differences between oceans and enclosed and coastal sea conditions, and the problems to be faced for their modelling. Specific examples are given with, wherever available, a quantification of the possible errors.

The meteorology defining winds for coastal and semi-enclosed water bodies increases in complexity from its deep water, open ocean counterpart. Synoptic-, meso- and micro-scale meteorological features have often a larger, impact on the coastal wave climate than they do in a deep, unrestricted open ocean body. Approaching the coastal domain, factors such as the land/sea interface, effects resulting from orography (differential elevation changes, e.g. mountains, cliffs), and sheltering effects (e.g. natural vegetation coverage, structures) may transform a coherent field into a complicated structure. Diurnal oscillations resulting from land-sea breezes will cause daily oscillations and directional shifts (see, among others, Stockwell et al., 2004, and Gemmrich and Garrett, 2012). In the upper latitudes williwaws (katabatic process) originate in the snow and ice fields of the coastal mountains where the building up of high density cold air causes the air to flow 
downwards warming adiabatically as it descends at increasing speed. A similar effect, although at much warmer latitudes, has been reported by Langodan et al. (2014) in the Red Sea. Pierson (1983) suggested many more examples in the scaling of the motions in meteorology, while Fujita (1981) cited 14 different ways to categorize these various scales. Cavaleri et al. (2012) qualitatively summarize the processes that characterize the air-sea interface.

Unlike in the open ocean, the coastal and inner sea domains are often bounded in up to three of their four directions. These regions are bounded by land masses, by a frequently limited depth (at least close to the coast), and in some cases by large-scale current patterns, (e.g. the Gulf Stream and the Florida Current). All of these will have an impact on the air-sea interface, hence affecting the local winds or the wave conditions. Along the US east coast, Appel et al (2005) objectively quantified the climatology of the Carolina fronts. These systems are the result of dominant high pressure systems located in the northeast of the US, the Gulf Stream providing the source of heat and moisture, and the mountains assisting the southward advance of cold air. This leads to the creation of a natural baroclinic zone near the coast, called a Cold Air Dam (Doyle and Warner, 1993). In structure these systems resemble shallow quasi-stationary or warm fronts with temperature differences that can reach $10^{\circ} \mathrm{C}$ over short distances on the order of $100 \mathrm{~km}$ or less (Bosart, 1975). These systems provide a source of surface convergence and vorticity that often lead to the generation of cyclogenesis. The classical and most notable miss-forecast is an early degradation of the Cold Air Dam and the consequent migration of the front inland. This can result in temperature errors up to $10^{\circ}$ and wind direction differences of up to $90^{\circ}$.

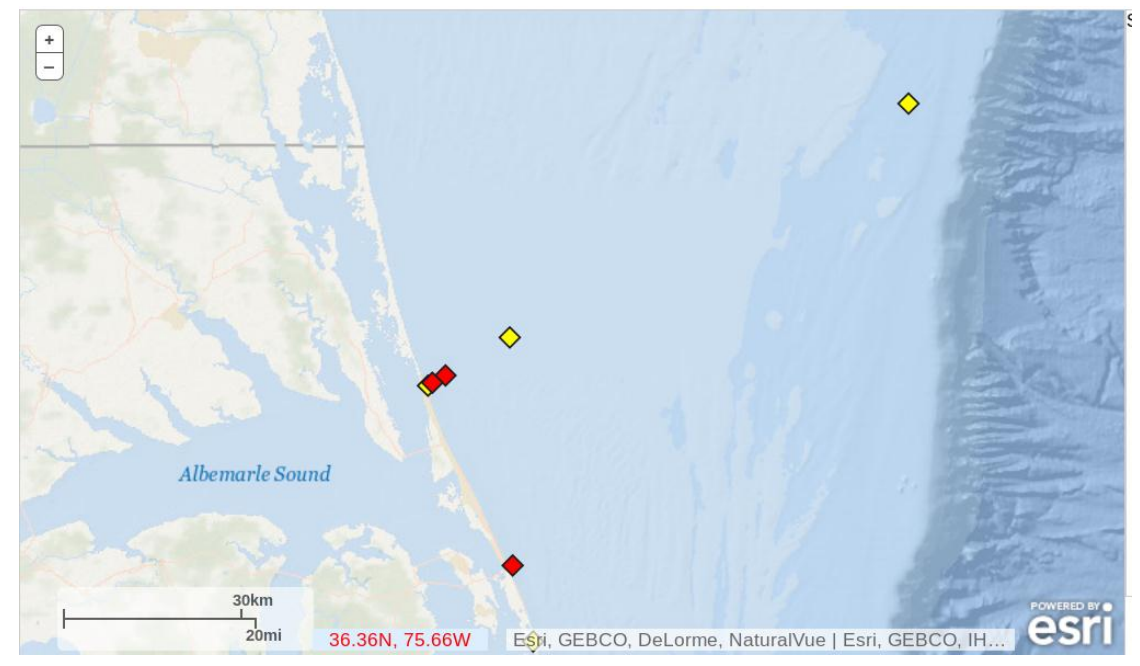

282 Figure 2.1 -Location of the USACE Field Research Facility on the outer banks of North Carolina, USA. The symbols show the positions of the pier (561 m long) and the measuring buoys. 
285 Apart from these "synoptic" examples, the coastal zone is frequently characterized by strong 286 gradients of the surface wind fields. With the recent push toward wind energy resources there have 287 been many field campaigns monitoring wind in the extended coastal zone, the related effects of 288 atmospheric stability, and the land-sea breeze systems (Barthelmie, 1999, Smedman et al, 2003, 289 Frederickson and Davidson, 2003). In this respect an almost permanent study has been and is the 290 one carried out at the USACE Field Research Facility located on the same US east coast (see Figure 291 2.1). There have also been multiple field experiments carried out in the area (SWADE, Wang et al., 292 1994; DUCK94, Birkemeier and Thornton, 1994; SHOWEX, Graber, 2005, Plant et al., 2005). Here 293 continuous data were obtained from two relatively close operational sites. The land-based NOS 294 Station DUKN7 is located at the end of the pier $(\sim 561 \mathrm{~m}$, at $15 \mathrm{~m}$ height, 295 http://tidesandcurrents.noaa.gov/stationhome.html?id=8651370 ). The offshore site is the 296 NOAA/NDBC 44014 buoy (http://www.ndbc.noaa.gov/station_page.php?station=44014) located 297 approximately $95 \mathrm{~km}$ from the coast in about $95 \mathrm{~m}$ depth. The anemometer is at $+5 \mathrm{~m}$ above the sea 298 surface.

300 It is instructive to conduct a qualitative examination to see how similar or different the winds are at these two sites. Both the winds have been transformed to equivalent neutral stable $10 \mathrm{~m}$ wind estimates using the $\mathrm{z}^{1 / 7}$ law ( $\mathrm{z}$ height above the sea). Together with neglecting the actual air-sea stability conditions, this is a crude approximation, but that we consider suitable for some general considerations. Even looking at the sustained wind cases only, when both the recorded winds are basically depending on the general synoptic situation, we find differences that cannot be justified by the distance between the two stations. On average the offshore winds are 5\% stronger. However, the situation is strongly direction dependent. In Figure 2.2 the comparison, as wind speed scatter diagrams, is shown for four incoming directions choosing two $120^{\circ}$ sectors centred on the perpendicular to the coast ("sea" and "land" respectively) and the two remaining $60^{\circ}$ sectors for winds loosely parallel to the coast. Then we see that the above $5 \%$ is the average of quite different situations. For "north", "south" and "sea" there is a limited positive difference pier-buoy that

312 however we consider within the above mentioned approximation. The large difference, 15\%, buoy313 pier is for offshore blowing winds. Note in particular that the differences increase for the larger 314 value range. The possible explanation is the different surface friction on land (soon to be discussed) 315 and the sea. This implies that, when wind enters the sea, there is the development of a new surface 316 boundary layer and a progressive increase of the surface wind speed, reaching the new higher 317 equilibrium value after a few tens of kilometres. Cavaleri and Bertotti (1997) provide evidence of 318 this process statistically analysing the winds out of the meteorological model of the European 
319 Centre for Medium-Range Weather Forecasts (ECMWF, Reading, U.K.). In the case of Duck the 320 effect is partly unexpected because Duck is on the North Carolina outer banks, with a relatively 321 narrow strip of land enclosing a large lagoon. So there is not really land onshore of the pier. 322 However, the much younger wind waves created by the sustained wind in the lagoon offer a much 323 greater stress to the blowing wind, not as much as on land, but certainly more than the well324 developed waves $95 \mathrm{~km}$ offshore. Differences exist also on direction and, remarkably, mainly for 325 the offshore blowing wind (for the other directions the values are more similar). In this case, the most numerous one, there is on average a $20^{\circ}$ counterclockwise turn by the time the wind reaches the buoy. Speculation into this disparity can only be made on the base of the local geography. However, the fact that we need to analyze the local reasons for the onshore-offshore differences is a strong indication that the wind characteristics in a given area are locally specific and need therefore to be carefully analysed place by place if accurate model estimates are required.
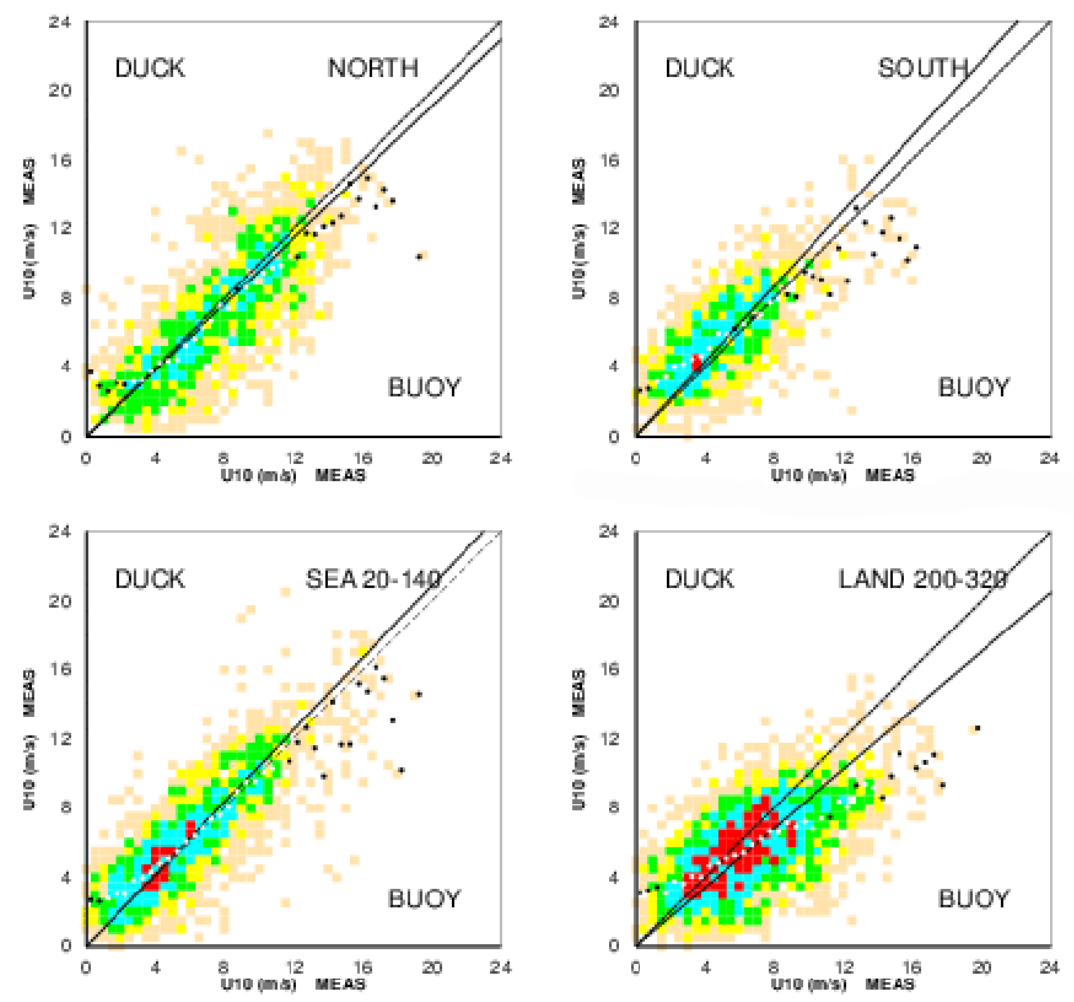

333 Figure 2.2 - Comparison between the wind speeds measured at the buoy $95 \mathrm{~km}$ off the North 334 Carolina, US, outer banks and the meteo station at the end of the Duck $561 \mathrm{~m}$ long pier. The comparison is shown for different incoming directional sectors. The coast is turned about $10^{\circ}$ counterclockwise with respect to the north-south direction. All the values moved to neutral conditions ten meter height. Incoming directions are considered. 
339 Another instructive comparison is among the winds recorded at three different stations in the

340 Northern Adriatic Sea (Figure 2.3). The stations are the offshore oceanographic tower of the 341 Institute of Marine Sciences (ISMAR, Venice, Italy), $15 \mathrm{~km}$ from the coast in $16 \mathrm{~m}$ water depth, the 342 anemometer at a local minor airport, $100 \mathrm{~m}$ from the beach on a narrow island bordering a large 343 inner lagoon, and the instrument at the Venice international airport, about $10 \mathrm{~km}$ from the sea 344 border, but at the inner edge of the cited lagoon. The three positions are marked in panel c. Long 345 term experience (see Cavaleri, 2000) shows that in any wind condition the tower wind speed is 346 substantially larger than the "land" data, with a progressive decrease towards "inland". The "." 347 stress that this is not simply due to a different surface friction on the sea and on land. Indeed, 348 similarly to the Duck case, with the exception of the narrow island where the minor airport is 349 located, the wind is blowing on the water of the lagoon till the international airport. The sirocco 350 wind, the one responsible for the floods of Venice, blows perpendicularly to the local coast. 351 Nevertheless differences up to $30 \%$ have been found with respect to the tower data. Again the point, 352 similar to the Duck case, is that the shallow water of the lagoon (average depth $<1 \mathrm{~m}$ ) and, to a 353 minor extent, the decreasing sea depth towards the beach imply a lot of breakers (waves in the 354 lagoon are very young because $c_{p} / U_{10}$ is small, with $c_{p}$ the peak phase velocity and $U_{10}$ the $10 \mathrm{~m}$ 355 wind speed). This leads to an increased surface friction, hence slowing down of the ten meter wind 356 speed.

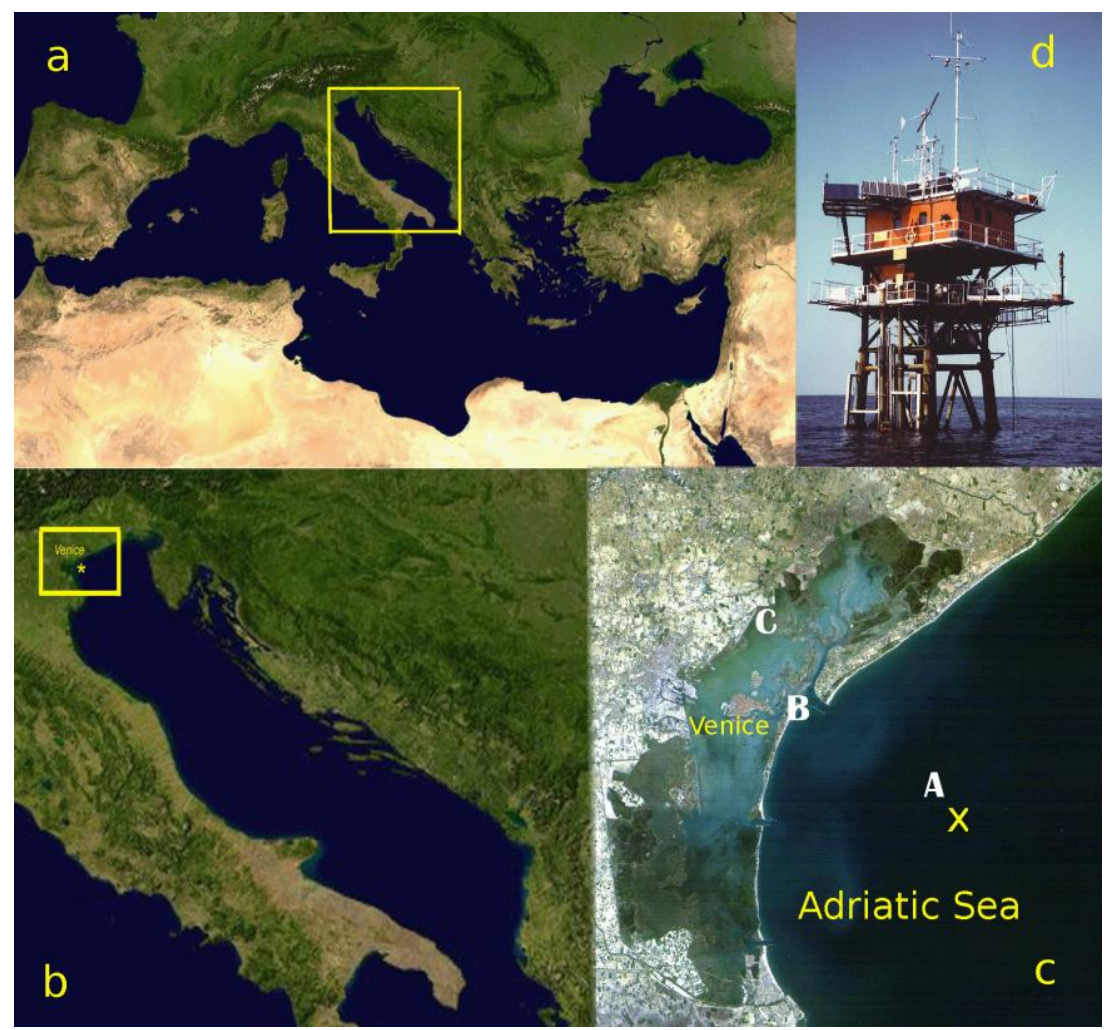


359 Figure 2.3 - a) Mediterranean Sea, b) Northern Adriatic Sea, c) the area of, and in front of, the

360 Venice lagoon. b) and c) progressive enlargements of the framed area. A, B, C mark the positions of 361 the ISMAR oceanographic tower and the two meteo stations cited in the text. d) the oceanographic 362 tower.

364 The differences between offshore (at the tower position) and close to the coast become even more 365 marked with the classical bora, a strong gusty wind blowing from north-east parallel to the coast 366 (see Figure 2.3). The across variability of the wind speed is also enhanced by the jet characteristics 367 of the bora, hence with a strong transversal gradient. This wind blows also parallel to the larger dimension of the lagoon $(50 \mathrm{x} 10 \mathrm{~km})$. Beside the wind speed reduction, in these cases the local extended surface stresses due to the extreme very short choppy sea are manifest in the water level in the lagoon, when the level at its southern end turns up to be up to one meter higher than at the other extreme.

More in general, whenever the wind passes from land to sea, there is a progressive decrease of the surface friction felt by wind, hence an acceleration of the wind speed till the new equilibrium value. The problem, as cited above, is that the meteorological models are too slow in reacting in this respect, with a consequent underestimate of the wind speed in the first 100 or $200 \mathrm{~km}$ off the coast, depending on model resolution (the higher, the better). This sorted out a long standing problem of explaining why, with offshore blowing winds, on the US east coast the offshore buoys data were showing correct model wind speeds, but underestimated wave heights. While carefully verified for ECMWF, the problem seems to be, albeit at different levels, a feature of most meteorological models. The only practical solution seems to be an increase of the model resolution, that leads to both a decrease of the spatial extent and of the level of the underestimate.

All this is evident, although not the only reason, when comparing the performance of the ECMWF global meteorological model in the ocean and in the Mediterranean Sea (an example of inner, although not small, basin). Cavaleri and Bertotti (2006) have explored how the mean and maximum values of the wind and wave fields vary on average with the resolution of the meteorological (and corresponding wave) model. Their results, updated with the latest resolutions, are shown in Figure 2.4. The horizontal scale shows the spectral resolution TL, corresponding to $40,000 /(2 * \mathrm{TL}) \mathrm{km}$ spatial resolution. (TCO is the latest model with $9 \mathrm{~km}$ resolution; see Malardel et al., 2016, for

391 details). We comment here on the meteorological models. The problems with waves will be dealt 392 with in the next section. 

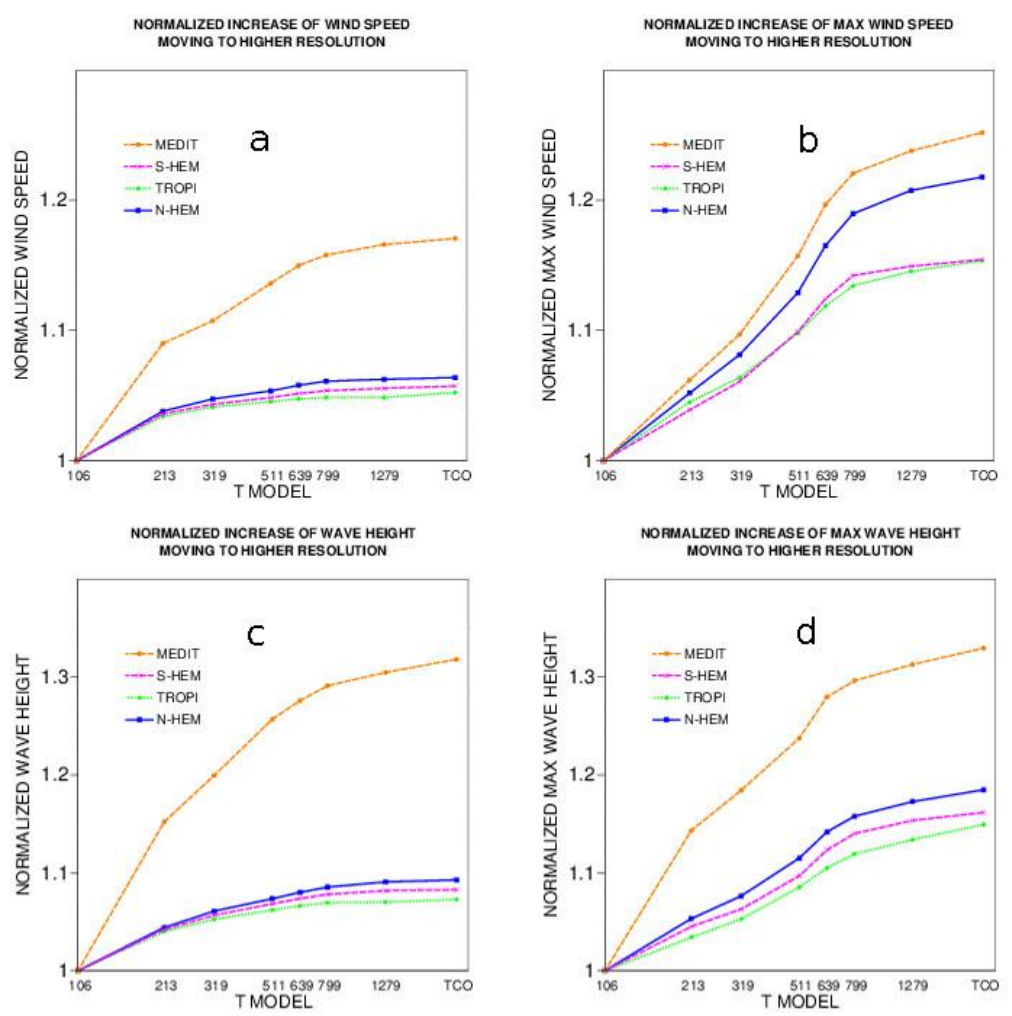

395 Figure 2.4 - Average increase of the a) c) mean and b) d) maximum wind speeds and significant wave heights in the northern and southern hemispheres, in the tropics and in the Mediterranean Sea. Upper panels for wind, lower panels for waves. The horizontal scale shows the spectral resolution of the ECMWF meteorological model (see text for details). For each resolution the results are normalized with respect to the T106 ones. The wave model resolution for these tests was the same till T511, then progressively increased parallel to the resolution of the meteorological model, up to the present $14 \mathrm{~km}$.

It is clear that, while the mean values (panel a) in the oceans (northern and southern hemispheres, plus the tropics) are practically asymptotic, the ones in the Mediterranean are still climbing (but note the compressed horizontal scale in the upper resolution range). This strongly indicates that we are still not close to a fully satisfactory solution for the inner seas.

408 The conclusions are slightly different for the maximum values in the field (panel b). Here, although different from basin to basin, all the maxima were 'exploding' moving to higher resolution, a strong indication that till T799 (25 km resolution) we were not yet able to pick up the physics and the

411 processes at the heart of the storms and hurricanes. Beyond this resolution we seem to be 
412 approaching the solution, although we still see an evident increase when, as done in March 2016,

413 the ECMWF resolution passed from 16 to $9 \mathrm{~km}$.

415 On a more local scale the orography of the coast can have dramatic effects on the local wind field. 416 A classical case is the presence of a coastal jet running parallel to a coast. Barrier jets occur when a 417 stable onshore flow interacts with a mountain barrier, but the flow is too stable to rise over the 418 barrier. The dynamic response is for the ascending air to cool and generate a hydrostatic positive 419 pressure perturbation which forces a turning of the wind (to the left) along the barrier (in the northern hemisphere - the other way around in the south). This flow is then rotationally trapped against the barrier and a barrier jet results (Winstead et al., 2004, and Loescher et al., 2006).

In the case of an offshore flow the local mountains and valleys lead easily to strong jets that exit the coast as strong concentrated flows. Classical examples are the Tihuano winds in the gulf of Tehuantepec (Mexico, see Garcia-Nava et al., 2009), the mistral on the Mediterranean French coast, the mentioned bora in the Adriatic Sea along the valleys that cut the Dinaric Alps of Croatia, and the Panama jet in the Colombia Pacific (Portilla et al., 2015). Most of these winds have been studied for many decades. Gap winds, coastal wind jets, or Bernoulli winds have been studied, among others, by Mass et al. (2014), Isoguchi and Kawamura (2007) and Zingone and Hufford (2006) using Synthetic Aperture Radar (SAR) images. These complex features can extend outward in the ocean for hundreds of kilometres, resulting not only in lobes of intense wind speeds, but also in large transversal gradients in the wind speed distribution.

Embedded in coastal wind records is a diurnal oscillation in the winds caused by land-sea breeze effects. These cells originate from the differential temperature gradient caused by the land and water heating or cooling at different rates. During the day sea breezes (onshore flow) will result; during the night land breezes (offshore flow) will be in control. It has been documented that the seaward extent of these cells ranges from kilometres to over $100 \mathrm{~km}$, see, e.g., for a large extent example Langodan et al. (2014). Gille et al. (2005) found that the strength of the diurnal wind cycle is statistically significant reaching far into the deep oceanic basins of the world. This phenomenon

441 has been studied, modelled (Jiang, 2012; Steele et al., 2013; Colby, 2004), and quantified in point 442 source measurements (Tsujimoto and Koike, 2013; Barthelmie et al, 1996; Zhu and Atkinson, 443 2004), or mining daily satellite wind observations (Gille et al, 2005). The intensity of the sea 444 breezes, up to $10 \mathrm{~ms}^{-1}$, and their extent in the coastal seas imply that they should be properly 445 represented in meteorological models. For the more limited extents, order of ten kilometres or so, 
the global models have an obvious problem of resolution. While the solution is in local high resolution modelling, care is required in choosing the optimal balance (for what breezes are concerned). Indeed the effects of grid resolution have been estimated by Colby (2004). The surprising conclusion from this study is that beyond a certain resolution a smaller grid spacing produced larger errors when compared to observations. This is due to the so-called 'double penalty effect', i.e. when the details represented by the model are not supported by sufficient measured data. In practice the model is physically sound, but not deterministically correct.

We have cited above how the local wave characteristics can affect the local wind conditions. More in general, the land/sea interface has been studied to determine changes in surface roughness, momentum transfers, and ultimately leading to a net change in the growth characteristics of windgenerated waves. The transfers of momentum across this interface is very complex (see, e.g., Janssen, 1991, 2004), varying on temporal and spatial scales spanning orders of magnitudes. Wave age (defined as $\mathrm{c}_{\mathrm{p}} / \mathrm{u} *$ or $\mathrm{c}_{\mathrm{p}} / \mathrm{U}_{10}$, where $\mathrm{u} *$ is the friction velocity) is the key parameter in establishing the kind and level of interaction across the interface. This is particularly intense, in both directions, between a generating wind and a young wind-sea. Therefore the related complexities are further increased in the coastal and inner seas. In arbitrary water (defined as non-deep water), wave related mechanisms, like shoaling, refraction and depth induced wave breaking will change the wind characteristics. Sun et al. (2001) found that in an offshore blowing wind the stress over the coastal water can be influenced by a strong turbulence advected from land. Further studies on coastal airsea fluxes (Frederickson and Davidson, 2003) found these regions to be far more complex than their deep water open ocean counterparts.

The possible interaction with the underlying wind fields opens a full perspective of possible interactions, that we will partly deal with in the section on coupling. A case of concern when working on a coastal area facing a large enough expanse of water is the possible presence of swell propagating towards the coast and against the local wind and wind-sea. These conditions have been well illustrated by both Flamant et al. (2003) and Garcia-Nava et al. (2009), working respectively in

474 the Gulf of Lion with mistral wind (North-Western Mediterranean Sea) and the Gulf of 475 Tehuantepec (Pacific Mexican coast) with the mentioned Tehuano winds. Both the studies have 476 highlighted the role of swell in affecting the local roughness length evolution with fetch, the growth rate of the wind sea, and consequently the spatial evolution of the offshore directed young wind sea. 
479 All the mentioned effects, related to coast or to the interaction with especially young waves,

480 become more and more relevant the more enclosed is the basin under consideration, in the limit 481 considering a fully enclosed basin, as for instance the Black Sea in Europe or the Great Lakes of 482 North America (Michigan, Superior, Huron, Erie, Ontario). One could think of these lakes as being 483 small-scale deep ocean bodies. The net effects of these systems on a wave climate are visually 484 evident. The coastal boundary layer and the land-sea breeze now surround the entire domain 485 effectively altering the wave climate. These fluctuations are more difficult to detect when masked 486 by larger-scale synoptic systems. Although a substantial number of studies have been performed on 487 wind and waves in lakes, most of them have been focused on wind-wave growth (Bretschneider, 488 1958; Donelan et al., 1992; Young and Verhagen, 1996) and not on the atmospheric forcing.

490 Because of the related strong economic interest, the Great Lakes have been and continue to be a 491 viable region to study. Point source buoy sites (approximately 15 sites, NOAA/NDBC and 492 Canada/MEDS) and shore based meteorological stations surround the perimeter of the lakes making 493 them a very practical region to study winds and wave generation. As a whole, they also encompass 494 many different meteorological events. Apart from the conditions associated to large scale synoptic 495 systems, one of the characteristics observed in these enclosed systems is the rapid acceleration of 496 cyclogenesis. This happens when there is a strong positive difference between the water and air 497 temperature, typically in the Fall, leading to an intense exchange of heat, in so doing fuelling the 498 possible rapid development of the storm. Typical examples happen in the North-Western 499 Mediterranean Sea and also in the Great Lakes. An example, among the many available, is given by 500 the storm of 23 September 1989 when, according to both model and measurements, over four hour 501 duration the wind speed increased from about 6 to nearly $20 \mathrm{~ms}^{-1}$ with a significant wave height 502 increase of nearly $5 \mathrm{~m}$ over a similar period. Also, during a sailboat race from Port Huron (Lake 503 Huron) to Mackinac a similar situation occurred (Burke, 2008), forcing almost half of the boats to 504 withdraw because of a rapidly developing storm system that was miss-forecast, 505 (http://www.bycmack.com/history/1985_Port_Huron_to_Mack_Race_by_John_Burke_(2008).pdf).

506 Neither of these cases were associated to frontal systems. They were both local, rapidly developing 507 storms. This makes them much more difficult to forecast, as repetitive experience has clearly 508 shown.

510 One of the characteristics of more or less isolated bodies of water is their immediate reactivity to 511 the changing meteorological conditions. Of course this holds for waves, an enclosed sea lacking the 512 longer term memory of the oceanic sites. For instance, this is again typical of the Great Lakes. 
513 Jensen et al., (2012) quote a 22 day period during which in Lake Michigan fourteen wind shifts of

514180 degrees occurred, none of them associated to sea or land breezes. Each time the wave system 515 reacted accordingly, with practically no memory of the previous conditions.

517 Still about waves (that we will discuss more in the next sections), there is a diffused tendency to 518 believe that the stormy wave conditions possible in an enclosed sea or a lake are much lower than in 519 the ocean. While this is true for the most extreme ocean storms, we should not forget that it does not 520 take $1000 \mathrm{~km}$ or more to build a severe storm. In the two documented (for their consequences) 521 storms in the Adriatic Sea, 4 November 1966 and 22 December 1979, that led to the two most 522 disastrous floods of Venice, Cavaleri et al. (2010) have estimated significant wave heights up to 12 523 meter on a mere few hundreds of kilometer fetch, reduced to "only" 8 meter when approaching the 524 Venice coastline, mostly because of bottom induced breaking. While no measured wave data exist 525 for either storm, the damage to the tower (see Figure 2.3) present after the 1979 event (not there in 526 1966) was a clear proof that during the storm the wave crests had repetitively passed above $9 \mathrm{~m}$ 527 above the mean sea surface. In Lake Superior the well documented sinking of the Edmond 528 Fitzgerald (Hultquistet al., 2006) during a November 1975 storm exemplifies the effect of rapid intensification of cyclogenenis and the consequent wave conditions occurring in the Great Lakes 530 that proved to be very deadly. No available buoy data were available to quantify the degree of 531 accuracy in the model estimate (7m, Hultquist et al., 2006). However, twenty three years later a 532 near identical storm entered the Great Lakes basin. Also this time only one measurement site was in 533 operation during the event, located in the southern part of Lake Michigan. Still not on the most 534 intense area of the storm, peak significant wave height conditions of over 6 meter were measured 535 (Jensen et al., 2012).

Having listed the problems we encounter when modelling wind and wave conditions in the coastal and enclosed seas, it is fair to recall the accuracy (see Chapter 1) presently achieved in the global models. However, the cited statistics hold on a global basis. In enclosed seas, particularly if affected

540 by local orography, the performance is appreciably inferior. For instance, in the Adriatic Sea 541 Cavaleri and Bertotti (2004) must regularly enhance the ECMWF wind speed to get the correct 542 corresponding values (compared with satellite data) to be used with the local wave modelling 543 system. The wind enhancement factor, decreasing with the progressively improving resolution, is 544 still at 1.16 notwithstanding the $9 \mathrm{~km}$ resolution of the present ECMWF model. The obvious 545 solution is high resolution limited area modelling. The system Nettuno (wind and wave forecast, a 546 cooperative effort of the Italian Meteorological Service and ISMAR) with $7 \mathrm{~km}$ resolution in the 
547 Mediterranean Sea, boasts a 1.00 best-fit slope for the model $\mathrm{U}_{10}$ vs measured data. However, at a

548 keen analysis the corresponding figure for the various sub-basins varies from 0.92 to 1.09 (Bertotti 549 et al., 2013). These are typical figures for enclosed seas, showing the difficulty of properly 550 modelling the various effects and coastal influence we have rapidly described.

552 As a summary of this section, meteorological effects in coastal and enclosed water bodies can and 553 do often become quite complex. It is as if we had a synoptic-scale (cyclogeneses, pressure systems, 554 frontal passages) on top of which smaller scale features keep adding or subtracting from the existing 555 conditions. We enter a regime where land/sea boundaries actively affect the flow patterns, and 556 orographic effects from large- (mountains) to small-scale (buildings and vegetation) play an 557 important role in the overall wind field. In addition to this, daily heating of the land can produce 558 diurnal oscillations in the very local winds or land-sea breezes. Thermal effects can generate small 559 scale sea surface temperature gradients resulting in transient surface roughness regions. This in turn 560 will affect the estimates in the frictional velocity and very local scale wind-wave generation. Field, 561 laboratory and modelling studies have been performed isolating these mechanisms. In semi- or fully 562 enclosed water bodies, one could identify the meteorological conditions as a microcosm of its open 563 ocean counterpart. The meteorology and the mechanisms are identical; but what could appear as 564 small-scale in the deep open ocean, in limited costal area will effectively impact the winds 565 themselves. In part the modelling capability of some of these situations has been greatly improved 566 in our present day forecasting efforts, but we still have a long way to go.

\section{L.Cavaleri, J.-R.Bidlot, and W.E.Rogers}

luigi.cavaleri@ismar.cnr.it

\section{2 - Open seas versus enclosed basins}

where we point out the problems with modelling waves in enclosed and coastal seas and the basic reasons for this condition. A deeper look into the related physics is in the following Section 2.3.

In the previous Section 2.1 we have highlighted the difficulties we encounter with properly defining the meteorological conditions in coastal areas and enclosed seas. As expected, this is not the only problem. Also wave, and more in general oceanographic, modelling faces new challenges. In this section we give a brief overview of the conditions that give rise to the differences between wave 
the consequent limitations. A more physical interpretation of the various aspects implied is given in the next Section 2.3.

584 In Chapter 1 we have pointed out the high level of accuracy of wave modelling in the oceans. 585 Performances with a few percent errors in the significant wave height, scatter indices of $10 \%$ or 586 slightly more are what is expected for the first day or few days of forecast from the best meteooceanographic operational centres. Of course this is the general view, and errors occur, for example with exceptional events where the physics of the meteorological and wave models may be pushed to their limits. Also, considering the evolution of a storm, the error can be less related to the estimation of modelled quantities than to where and when the storm occurs. A 5\% error in the speed of a cold front will misplace it by about $100 \mathrm{~km}$ and three hours during the course of a three-day forecast.

592 While on the whole the statistics will be good, at specific times and locations the evidence will be 593 quite different.

595 One difficulty in the open ocean is the full specification of the wave conditions, generally characterized by a wind sea and one, or more often a few, swell(s). While the $H_{s}$ statistics may be favourable, we may find large differences in the spectral distribution of energy (this subject is discussed also in Section 2.5, and more extensively in Chapter 4). In a way the accuracy depends on the purpose. For instance, a ten-day towing of an oil rig across a large expanse of ocean may judge as dangerous (or at least relevant) certain errors in $H_{s}$ and swell forecast, while the same errors may 601 be considered irrelevant for an oil tanker. In any case the fact that we indeed consider as usable ten602 day forecasts is in itself a marvel. Having become accustomed to the almost constant high quality, 603 we should not forget that at a given time, what in ten days will be the crucial meteorological factors 604 for the local conditions may be at the moment almost half a globe away.

606 Table 2.1 - Performance of the distributed NCEP wave forecast system versus 50 NDBC coastal 607 buoys in the Gulf of Mexico and US East coast. rmse is root mean square error, SI scatter index. 608 Error metrics are for significant wave height.

\begin{tabular}{|l|c|c|c|c|}
\hline forecast (h) & 24 & 48 & 72 & 96 \\
\hline rel bias (\%) & -5.6 & -4.3 & -4.2 & -4.2 \\
\hline rmse (m) & 0.24 & 0.27 & 0.31 & 0.42 \\
\hline SI & 0.20 & 0.22 & 0.26 & 0.35 \\
\hline best-fit slope & 0.88 & 0.88 & 0.88 & 0.86 \\
\hline
\end{tabular}


611 Once we move to the coastal and inner seas, the statistics of the global model results are not so 612 good. See for example Table 2.1, reporting the performance of NOAA operational WAVEWATCH 613 III Multi_1 model (soon to be described in Section 2.6) at all coastal NDBC buoys in the Gulf of 614 Mexico and the US East Coast. The performance can be considered good for coastal waters, but 615 certainly not up to the level of the global model results. The persistence of the underestimate (on 616 average $4.5 \%$ ) with the extent of the forecast shows the consistency of the global meteorological 617 model that is not letting the atmosphere gain or lose energy with time. However, the specific errors 618 of the details, e.g. the mentioned ones in space and time, are evident in the progressive substantial 619 increase of the scatter index. This is already relatively large, 0.20, at $24 \mathrm{~h}$ forecast (compare with 620 the 0.10 of the global model on the oceans from the statistics cited in Chapter 1), and then growing 621 with what one could judge almost an exponential rate.

623 Following the above argument, because in enclosed seas the dominant condition is wind sea, with 624 swell more rarely, if ever, present depending on the local geometry, any error in the driving wind 625 field implies a direct corresponding error in the wave conditions. This considerably shortens the extent of the useful forecast. But there are other reasons. Still referring to the global models, the resolution may, and in general will, not be sufficient to carve out the necessary details, both in the driving winds (think of orography) as in the geometry of the local basin. The common problem of a too slow catch up of the surface wind when passing from land to sea implies that the smaller the basin the stronger the corresponding underestimates will be (see Cavaleri and Bertotti, 2004, for a clear picture of the situation).

633 The obvious solution is to move to nested modelling, something useful both for (meteorological) 634 better describing the wind field as a function of the local orography and geometry, and (waves) 635 providing the correct geometry and bathymetry of the basin. Because there is a direct relationship 636 between the resolution of a model and the scale of the processes that we will be able to describe, a 637 higher resolution will make it possible to follow in greater detail the small scale events often 638 associated to enclosed seas. However, this higher sensitivity is not without cost. Nested models are 639 very sensitive to errors in the initial and boundary conditions. The cited (see above) error in timing 640 and location in the global models cannot be corrected by the nested grids that will inherit errors 641 from the larger grids. In some cases, as the Nettuno system in the Mediterranean Sea (Bertotti et al. 642 2013), an independent data assimilation system can be used. This avoids the influence of the global 643 model in the initial conditions, but, if the sea is not fully enclosed, the local model is still dependent 
644 on the global one for the boundary conditions. A large local area can be chosen to extend the

645 independence, but at the expense of the required computer time. In practice nested models do not 646 usually go further than three or four days, because on a longer forecast range they will be 647 completely dependent on the accuracy of the global model.

649 By increasing the resolution, we may be able to see many more details, and visualize more 650 processes at work, in the wave models, but more so especially in the meteorological models. These 651 details, as the small scale (order of kilometres) turbulence in the field, may look, and possibly are, 652 physically sound. However, this does not mean they are deterministically correct. The key point is 653 that simply we do not have the information for driving this, e.g. turbulence, with data assimilation. 654 Luckily, being integrated in space and time over the driving wind fields, waves typically low-pass 655 filter these oscillations, providing the output that duly reflects the larger scale pattern. Note however 656 that the obtained wave energy may duly and correctly represent the integrated effect of small scale turbulence (think of the effect of gustiness explored by Abdalla and Cavaleri, 2002).

On the fetch-limited conditions typical of the enclosed seas, the significant wave height $H_{s}$ is proportional to $U_{10}$ (see, e.g., Kahma and Calkoen, 1992), hence the $H_{s}$ errors associated with wind speed error are not so large as in the ocean, where for well-developed conditions $H_{s}$ tends to approach a square dependence on wind speed. On the other hand, the typical generative conditions are the most complicated ones for a model because of the various processes at work (generation, nonlinear interactions, dissipation, plus all the shallow water ones). This is immediately evident when, instead of the classical case of a straight coast and a perpendicular offshore blowing wind, we have a curved coast and possibly slanting (oblique) fetch generation. This also gives rise to the need for expanding the global and shelf sea operational observing networks to these near-shore locations, to be able to develop and validate operational models at these scales (see more on this in Section 2.5). As it will be discussed in Chapter 3, interactions with currents becomes a common issue in near-shore water where the wind and wave action leads often to currents of much larger intensity than what we usually find offshore, or also of different type, e.g. rip currents.

673 Summarizing the general view, to be detailed in the following sections, and particular in the examples of Section 2.6, perhaps the most critical information for wave modelling in the inner seas is the meteorological input that sets substantial limits to the extent of the usable forecast range. The statistics of the model performance are not as good as in the open sea, but still at a high usable level. This is on average. Detailed values for specific areas vary from almost perfect (best-fit slope close 
678 to 1.0) to large errors, the latter often depending on errors of the large scale model to which nesting 679 can be extremely sensitive. As is the case on a global scale, a useful approach to establish the 680 confidence in the forecast and the related uncertainty is the ensemble forecast, see Pezzuto et al. 681 (2016) and Bunney et al. (2016) for a detailed related study in the Mediterranean Sea. However, all 682 these results seem to vary amply with the application and the specific area. This is clearly 683 exemplified in Figure 2.4 (see the previous section) where we show the progressive increase, in the 684 oceans and the Mediterranean Sea, of the ECMWF mean and maximum wind speeds and significant 685 wave heights with the resolution of the operational meteorological model. Note that for these tests 686 the wave model resolution was the same till T511 (see Section 2.1), then progressively increased 687 with the meteorological one till the present $14 \mathrm{~km}$.

689 While the ocean mean wave heights are asymptotic to what (see the statistics of ECMWF and 690 NCEP cited in Chapter 1) appears to be an almost correct value, it is interesting to note how the 691 corresponding maxima are still climbing. This suggests that indeed the peaks of some storms are 692 characterized by very high spatial gradients. For our present purposes all this is exacerbated in the 693 Mediterranean Sea where we see that both the mean and maxima values are still climbing, a strong 694 indication that, as shown by repeated comparisons with satellite data, even at 9 and $14 \mathrm{~km}$ 695 resolution (for wind and wave respectively) we are still not close to the correct solution.

697 Though not specifically pertaining to the coastal situation, it is useful to consider the impact of sea 698 ice on waves and wave predictions. There are two different aspects where this impact is felt. One is 699 the variability in time of the extent of sea ice, hence in reverse the one of free sea where waves can 700 develop. The second one is the impact of ice in its various forms, from its early formation (grease 701 ice) to more or less dense ice floes and further to a compact pack, on the waves themselves. To 702 envisage the importance of sea ice, one need only imagine the implications of disregarding sea ice 703 when modelling regions where ice occupies some fraction of the basin. We do not need to think to 704 the retreating Arctic Ocean ice. Even inner or limited seas as, e.g., the Baltic Sea, Beaufort Sea, 705 Caspian Sea, or Nordic Sea show, and have shown in the past, ample seasonal variations. 706 Neglecting this would of course result in excessive wave generation, among other problems. On top 707 of the implied 'free' geometry of the basin, sea ice has a number of implications for wave modelling 708 in the ice environment. This will be dealt with in the next Section 2.3 


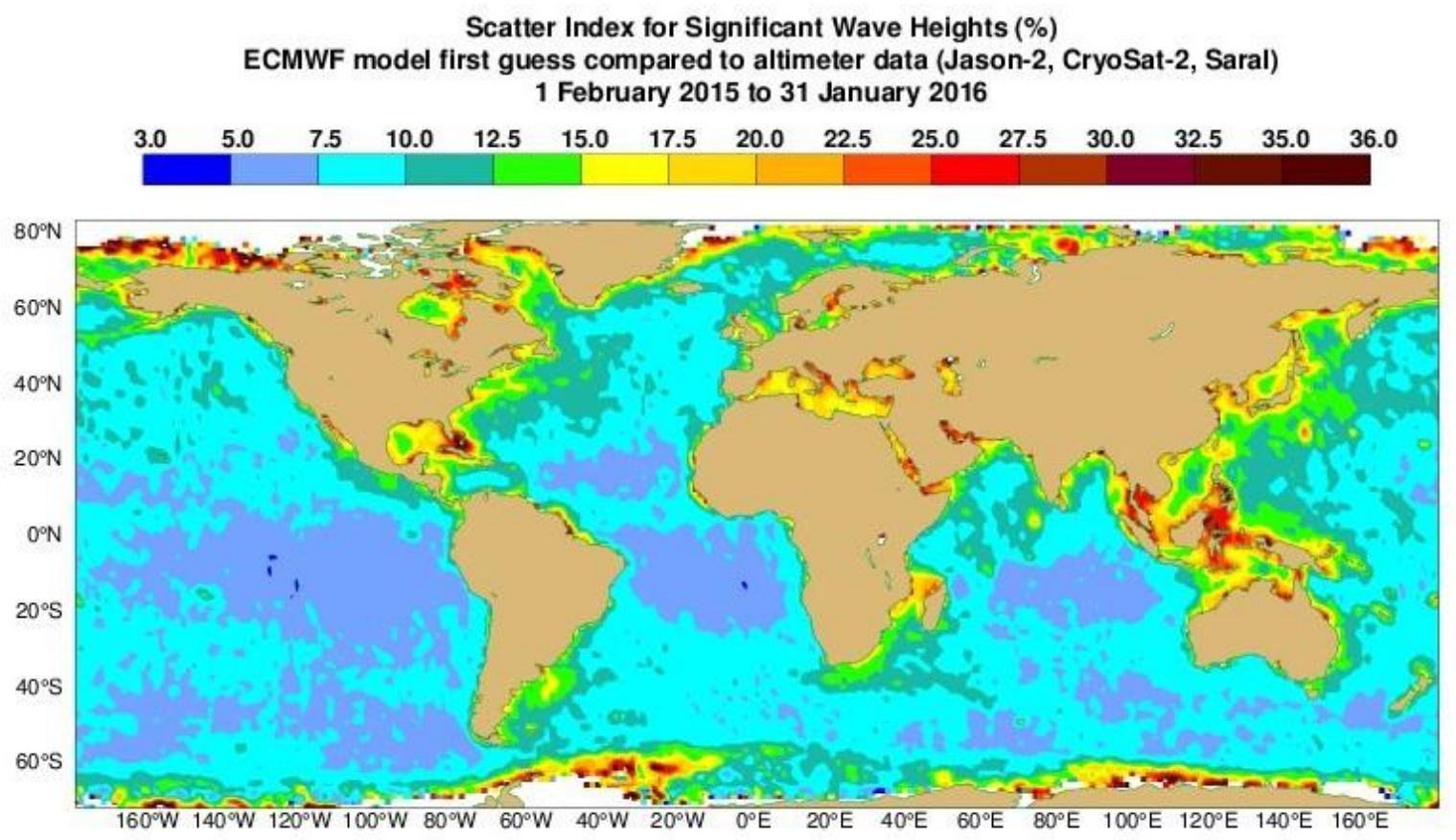

710 Figure 2.5 - Global distribution of the scatter index for the validation of the ECMWF significant 711 wave height first guess versus altimeter data. Note how all the coasts (especially those facing east) and inner seas are characterized by much higher scatter values than in the open oceans.

714 We close this quick and general survey of the "open seas versus enclosed basins" line of discussion 715 with a figure that beautifully summarizes the situation, making clear the level of performance in the 716 two different environments. An old saying goes as "a picture is worth a thousand words". This is 717 indeed the case here. Figure 2.5 shows the global distribution of the ECMWF scatter index (SI) for 718 the significant wave height first guess (i.e. before any data assimilation) compared to the Jason-2, 719 Cryosat-2, and Saral altimeters. Looking at the picture, we see at once how, with respect to the open oceans, the higher SI are practically a drawing of the continental borders. Note in particular the inner seas, as the Great Lakes, the Gulf of Mexico, the Mediterranean, the Red Sea, the whole area

722 between the Asia South-East and Australia, the area between Japan, Korea and China. Note the 723 large SI along the whole US North-East coast and, in contrast, how the west coasts fare in general 724 better. The obvious reason is the general west to east motion in the storm belts. There are exceptions. One is the Somalia coast, on the Horn of Africa, because the area is dominated by the south-west to north-east monsoon in summer and the opposite direction one in winter. The other 
exception is the North Sea, between U.K. and Scandinavia. Although subject to violent storms and acting as a semi-enclosed basin, the area is amply exposed to the North-Atlantic storms and swell, this link giving a higher reliability to the local forecasts, at least for waves entering the sea from north-west. This example will be further exploited when discussing data assimilation in Chapter 4 . Figure 2.5 has been obtained comparing with altimeter data. It could be argued that these data are not fully reliable close to coast, in so doing infirming the overall result. This is not the case. First ubiquitous altimeter data have been chosen to highlight the difference between ocean and coastal water. Then, of course with less data, similar results (Bidlot, 2017) have been obtained also, where available, for buoys.

At this stage the message is clear. We have a problem in the inner and coastal seas. The reasons why and the physics behind is what we deal with in the next and following sections.

When modelling waves in coastal areas or enclosed seas additional challenges emerge in comparison to open ocean wave modelling. The common factor of these two kinds of areas is the proximity of land resulting in relatively small-scale changes in bathymetry, currents and winds, whereas differences are mostly related to the amount of sheltering from externally generated waves. The interplay of all these factors requires a careful assessment of the significance of these processes on wave evolution. This knowledge can then be used to make proper choices in setting up a wave model for coastal applications, which become increasingly important in view of, e.g., the development of renewable energy resources or coping with coastal hazards due to sea level rise and climate change.

760 Good examples of complicated areas for wave modeling are the North Sea, the Gulf of Mexico, the 761 Bothnian Sea (part of the Baltic Sea) and the Adriatic Sea (a sub-basin of the Mediterranean Sea, see Figure 2.3). The North Sea is a partially enclosed sea with a decreasing depth while moving from north to south. Along the Danish and Dutch coast the water depth slowly decreases up to the 
coast-line, but along the east coast of England and along the Belgium coast many shallow ridges exist causing small-scale variations in wave characteristics. As the North Sea is connected to the Norwegian Sea and the Atlantic Ocean, occasional swell systems may also penetrate south-wards providing mixed sea states. Of particular interest for the Gulf of Mexico is the shelf break, and Gulf Stream-related currents. Both areas are also known for their violent storms or hurricanes causing storm surges and dangerously wave conditions of which the 1953 and 1961 storm surges in the North Sea and hurricane Katrina of 2005 are well-known examples. The Adriatic Sea is unique in its shape, a rectangular basin in which strong southerly scirocco winds may create large waves and dangerous storm surges at its northern end.

774 Of further interest is the transition to smaller spatial and temporal scales in coastal regions. This is particularly true for the North Sea which in its southern part is bordered by many shallow estuaries and tidal inlets of which the Wadden Sea is the most prominent feature. In these areas water level variations and current effects play an important role on wave evolution. A special feature of the Wadden Sea is the tide induced flooding and drying of sand banks and the occurrence of various muddy areas. Different spatial scales also occur in the southern part of the Bothnian Sea where thousands of islands ranging from a few metres to several kilometres in diameter make any modelling a real challenge. This wide range of spatial scales results in strongly varying wave conditions in relatively small geographical areas.

All these effects have implications for the proper choice of the physical processes to be modelled, the specification of external factors involved in forcing the wave model, the spatial resolution and numerical techniques in solving the wave (action balance) equation and the validation of model predictions. In the following sub-sections each of these aspects and their mutual interactions are discussed.

\subsection{1 - Depth effects}

A key difference between open ocean and coastal areas is water depth. As the water becomes shallower depth effects influence the evolution of the wave field. On the one hand depth effects influence the propagation of wave energy while on the other hand they affect the physical processes of wave generation, dissipation and non-linear interactions exchanging wave energy between different wave components. 
798 Spatial variations in depth influence the kinematics of the waves causing changes in the phase 799 velocity producing wave refraction, while changes in group velocity cause shoaling and a 800 steepening of individual waves. The spatial scale of depth changes is of importance for choosing 801 stable and accurate numerical methods for wave propagation. A good example is the treatment of 802 areas with steep gradients in bathymetry that are poorly-resolved by the model spatial resolution.

803 Such situations may require limiters to prevent unrealistic solutions, see for instance Dietrich et al. 804 (2013). This subject is discussed more thoroughly in the following Section 2.4. Though 805 computationally more expensive, applying sufficiently fine higher spatial resolution is still the best 806 option to properly resolve the gradients in the wave field (e.g. due to spatial changes in bathymetry 807 or currents). In case of explicit propagation schemes, this inflation of computational expense is 808 doubly true, because these schemes require that the time step size decrease in tandem with the 809 smallest geographic grid spacing. These limitations can be overcome by applying unstructured grid 810 techniques in conjunction with implicit schemes of which those by, e.g., Roland (2008), Zijlema 811 (2010) and Huchet et al. (2015) are good examples.

813 Depth effects have also consequence on the physical processes of wave generation by wind, 814 dissipation due to steepness or by depth limitations, and non-linear triad or quadruplet wave-wave 815 interactions. From the wave side point of view, shallow water effects may cause waves to become 816 steeper invoking breaking. Such sea state dependencies on wave evolution were discussed by Makin 817 (2002) and Babanin and Makin (2008) based on a field study in Lake George, Australia. They 818 identify various effects of which depth-induced wave breaking and steepening of individual waves 819 are most important for wind wave growth. Background swells may also affect the sea drag through 820 hydro-dynamic interactions in which shorter waves may experience enhanced breaking. This issue 821 is still not settled as found by Ardhuin et al. (2007) who did not find any effect of incoming swells 822 on local wave growth near Duck, North Carolina.

823 In deep water, breaking is associated with steepness. As waves enter shallow water, traditional 824 deep-water parameterizations for steepness-induced breaking are generally unable to predict the 825 strong enhancement of breaking rate by depth effects, so some "engineering solutions" are needed 826 to predict, for example, the variation of wave height across a beach profile. One of the most popular 827 approaches to this family of so-called "depth-induced" wave breaking formulations is the empirical 828 bore-based model of Battjes and Janssen (1978). The actual water depth is a key parameter in this 829 model and its performance is still surprisingly good (Salmon et al., 2015; Salmon and Holthuijsen, 830 2015). Progress in improving this model is slow as the physics of breaking is still poorly 831 understood. Ruessink et al. (2003) introduced the dimensionless depth $k h$, and Van der Westhuysen 
832 (2010) introduced the bi-phase to scale the dissipation rate. Salmon et al. (2015) suggest another

833 local scaling method by including depth, bottom slope and directional spreading. It is evident that

834 an accurate estimate of the total water depth is essential for a proper application of such breaker

835 formulations. Lastly, Filipot et al. (2010) propose a so-called "universal" treatment of breaking

836 which would, in principle, make it unnecessary to separately specify source terms for open-ocean

837 breaking (“whitecapping”) and surf zone (“depth-induced”) breaking.

839 Note that in all these formulations the local effect of wind is ignored. An order of magnitude 840 estimate of the involved energy fluxes puts the local wind input below the dissipation for only depth 841 effects. However, the problem is not in the amount of energy involved. Rather, there is the distinct 842 possibility that wind, if properly aligned, may favour the local shallow water breaking. The opposite 843 situation is also possible. A sufficiently strong reverse wind, i.e. blowing towards offshore, hence 844 against the incoming 'ready to break' waves, may lead to a reverse breaking, in so doing possibly 845 counteracting the "wish" of the wave to break shoreward. An example of this situation is in Figure 846 2.6. The next sub-section provides a compact view of the general situation in white-capping.

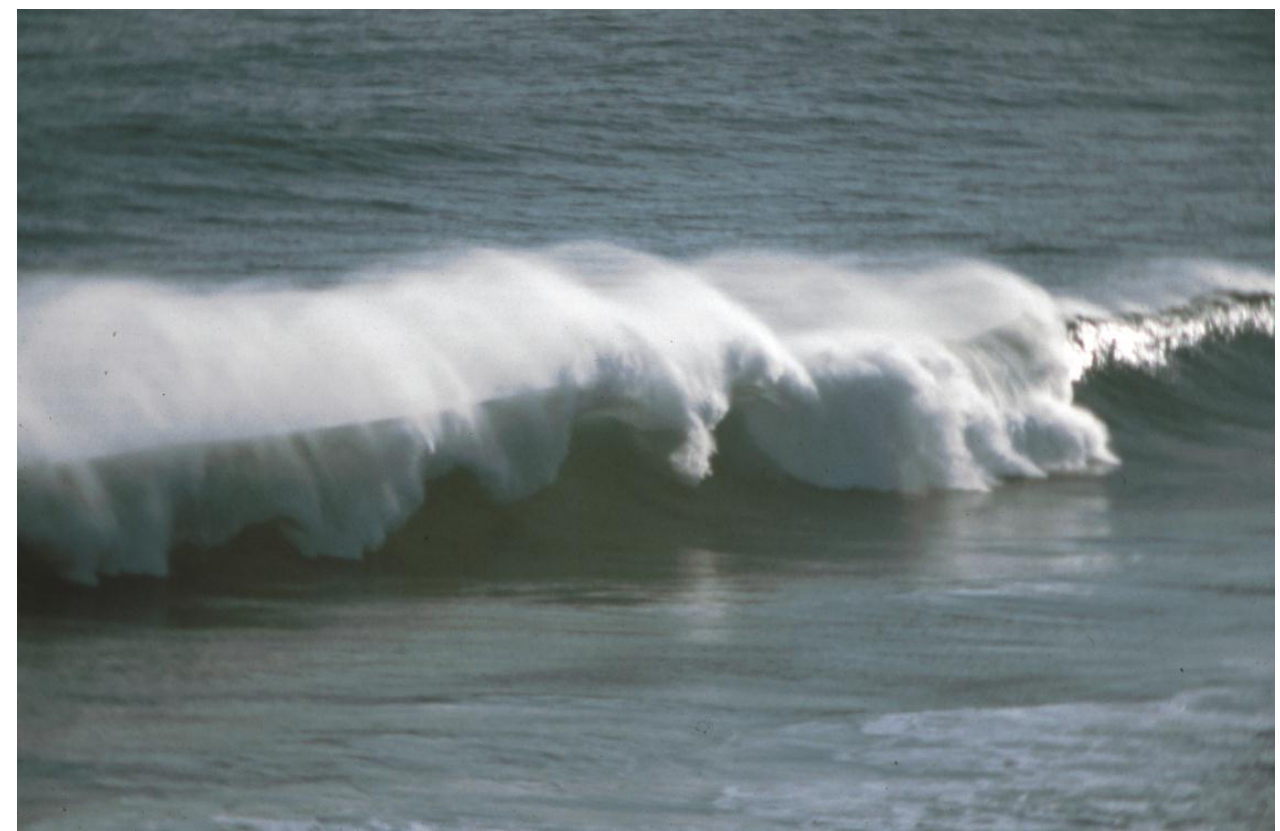

848 Figure 2.6 - Reverse breaking in shoaling waves due to adverse wind (photo by Luigi Cavaleri)

849 As shown by Herterich and Hasselmann (1980), the strength and spectral shape of the resonant non850 linear quadruplet transfer rate change as water becomes shallower. The consequences for wave 851 modelling in shallow water have hardly been explored as an accurate calculation of these 852 interactions is still not feasible for operational applications. A simple parameterisation of depth 853 effects has been included in the WAM model (WAMDI, 1988) based on an analysis of peak values 854 of the transfer rate as computed by Hasselmann and Hasselmann (1981). This simple method 
855 neglects the change in spectral shape in the non-linear transfer rate. A deeper problem is the 856 applicability of presently used formulation of the quadruplets, as strictly speaking they were derived 857 assuming homogeneity of the media in which the waves evolve (see, e.g., Rasmussen, 1998). 858 Moreover, Willebrand (1975) pointed to inconsistencies in the wave action balance equation in 859 inhomogeneous media. Smit (2014) developed new methods to handle these issues more accurately 860 in which the coherence of the wave field is taken into account by also keeping a balance of wave 861 phases. Both points of concern need further attention to improve model application in coastal areas.

862 In very shallow water non-linear triad wave interactions become important. These interactions 863 generate both lower and higher harmonics in the wave spectrum. These interactions can properly be 864 modelled in phase-resolving time domain model, like Boussinesq models (e.g. Madsen and 865 Sørensen, 1992, Kirby, 2003, Sørensen et al., 2004) or non-linear non-hydrostatic wave models 866 (e.g., Zijlema et al., 2011). In phase-averaged models only approximate methods have been 867 developed to redistribute energy within the wave spectrum. A well-known example is the Lumped 868 Triad Approximation (LTA) of Eldeberky and Battjes (1995), but its accuracy is poor. A fully 869 directional model has been described by Becq-Girard et al. (1999) known as the Stochastic 870 Parametric Boussinesq (SPB) model. A collinear implementation of the SPB model was developed 871 by Salmon et al. (2016) showing improved behaviour in comparison with the LTA method. A 872 fundamental problem occurs in phase-averaged models as the generated harmonics are treated as 873 free surface waves whereas they are linked with the primary peak of the spectrum.

\subsection{2 - White-capping and breaking}

876 Although not a process confined to shallow or enclosed seas, white-capping is still a problem for wave modelling. Granted some new insights and improved versions in the recent years, by and large

878 white-capping is still the tuning knob of most operational wave models. In framing the situation for enclosed seas, it is therefore convenient to summarize the related modelling situation.

880 In the early formulations by Komen et al. $(1984,1994)$ the white-capping dissipation was associated 881 to the spectral mean wave steepness. Implicitly the approach assumed a wind sea where the mean 882 steepness has a well-defined physical meaning. However, this parameter, once defined from the 883 whole spectrum, loses its original meaning, hence its usefulness, if, as commonly the case in the oceans, different wave systems, in practice including one or more swells, are present. Indeed, 885 although with some limitations, this can be the case in coastal and/or shallow seas.

886 The situation can be summarized as follows. The use of a mean-wave steepness causes problems in 887 mixed sea states (wind sea plus swell), as the dissipation rate is too high for the swell part and too 888 low for the wind sea part, causing an over-prediction of the wind sea part and an under-prediction of 
the swell part. Rogers et al. (2003) proposed an alternative weighting of the mean wave steepness to ameliorate the negative side effects, but this can only be regarded as a temporarily fix. More recent whitecapping formulations like those of Ardhuin et al. (2010) and Zieger et al. (2015) consist of a mix of a saturation based whitecapping formulation and a cumulative wave steepness effect on the

893 whitecapping dissipation rate and, more importantly, swell dissipation is treated separately.

894 A fundamental, but still ignored, problem is the relationship between white-capping and wind

895 speed. White-capping is strictly related to the energy and momentum input by wind on a generative 896 sea. As mentioned above, so far whitecapping formulations neglect the effect of wind on the 897 process. There is, however, accumulating evidence that wind effects cannot be disregarded for this 898 type of dissipation.

\subsection{3 - Bottom processes}

901 In shallow water the orbital motion of the water particles extends down to the sea floor. This leads 902 to interactions between the surface waves and bottom roughness elements of length scales 903 associated with sediment size and the characteristics of the orbits, down to the scale of the bed 904 ripples. One of these interactions is bottom scattering resulting in a local redistribution of wave 905 energy (Ardhuin and Herbers, 2002). A dissipative interaction is based on friction in the turbulent 906 boundary layer whose strength depends on the bottom conditions. Hasselmann et al. (1973) 907 suggested using an empirically based constant with different values for wind seas and swell. 908 Zijlema et al. (2012), however, argue that one value suffices in conjunction with a proper choice of 909 a wind drag formulation. A nonlinear formulations based on drag was proposed by Hasselmann and 910 Collins (1968) then simplified by Collins (1972). More complied, eddy viscosity model were 911 developed by Madsen et al. (1988). A complication is that in many circumstances the bottom 912 ripples characteristics may changes depending on the wave and current conditions. This requires 913 movable bed formulations which were addressed by Tolman (1992) and Smith et al. (2011). In 914 practise, the variations in bottom conditions in coastal areas make the a priori selection of friction 915 parameters problematic. As a consequence bottom friction is commonly part of the tuning 916 procedure, often specific for a given area, and spatial variations within a model grid are commonly 917 ignored.

918 In many coastal areas muddy bottoms exists. These consist of cohesive sediment or fluidized mud.

919 A problem with such bottom type is that it presents a rather different mode of wave dissipation 920 which is difficult to represent (e.g. Winterwerp et al., 2007; Holland and Elmore, 2008; Rogers and 921 Holland, 2009; Engelstad et al., 2013). Idealized models have been derived that are based on a two922 layer description of the water column in which surface waves drive internal waves at the interface 
923 between the nearly inviscid water and the highly viscous (and therefore dissipative) muddy bottom

924 layer. Muddy areas may also affect the growth of short waves (Trainor, 2009) or lead to extensive 925 sediment plumes (Engelstad et al., 2013). Mud rheology (characteristics including thickness, 926 viscosity, density, elasticity, and plasticity) is difficult to estimate even from in situ data, since the 927 mud changes when it is extracted from the water, and the depth of fluidization is highly non928 stationary, even when overall mud thickness does not change. Mud can also be transported of 929 course, and thickness is not uniform spatially, making this an intimidating problem for a modeller, 930 see the recent work by Samiksha (2015) and Shinu et al. (2017). Coupled wave-sediment models 931 are one approach for dealing with this, and can be applied to both cohesive and non-cohesive 932 sediments. The reliability of bottom boundary layer modelling is not well established, however.

933 A recent field of interest is dissipation by vegetation such as mangrove forests or salt-marshes 934 (Suzuki et al., 2011, Anderson and Smith, 2014; see also Samiksha, 2015). Such vegetation grows 935 in the inter-tidal zones along tropical and temperate coasts. Such areas may help protect vulnerable 936 coastal areas against wave attack and there is a recent trend to artificially create such areas, being an 937 example of "building with nature". The hydro-dynamics of vegetation fields are different from 938 bottom roughness elements. Still, the earliest attempts tried to model the dissipation using an 939 equivalent bottom friction value tuned to local circumstances. A more advanced approach is to 940 consider the vegetation as structural elements. A commonly method is to determine the drag by a 941 field of cylinders with a certain diameter and density (Dalrymple et al., 1984). This approach was 942 extended by Mendez and Losada (2004) to include specification of vegetation characteristics, but 943 also bottom slope and breaking waves. Suzuki et al. (2011) implemented this extended approach in 944 the SWAN model.

\subsection{4 - Sea ice}

947 In the previous section we have highlighted the importance of sea ice for the geometry of a basin 948 where waves can actually be generated. However, this is a simplified view because the different 949 forms under which ice is present on the sea imply different physical interactions with ocean waves. 950 Here we give a brief overview of the problems involved.

951 Historically, sea ice was treated in phase-averaged wave models by simplistic methods. In early 952 versions of WW3 and WAM, sea ice was treated as either open water or land, with the binary 953 selection based on ice concentration (e.g., Tuomi et al. 2011). In WW3, this was updated by Tolman 954 (2003) to a "continuous treatment" to allow partial blocking for partial ice cover. These methods do 955 not treat the effect as "dissipation" via the $S_{\text {ice }}$ term, but rather as a feature of the propagation 956 scheme. Further, they do not permit variation of dissipation rate with frequency, which is a clear 
defect given the intuitively obvious existence of such variation in the real ocean. High frequency waves are rapidly attenuated, while long period swell can penetrate hundreds of kilometres under a solid ice pack. More recently, WAM and WW3 have been updated to treat the sea ice as part of the

960 physics (see Doble and Bidlot, 2013, and Rogers and Orzech, 2013), a concept which was in fact 961 proposed much earlier (Komen et al., 1994), but never implemented outside of academic studies.

962 Sea ice can have a number of effects on the waves. The first, and perhaps most obvious, one is the 963 dissipation of wave energy. This depends strongly on the characteristics of the ice and on wave 964 frequency. The tail of the spectrum is rapidly attenuated also by small ice floes. Long period swell 965 propagating under the pack is attenuated by the strain induced into the undulating ice and the orbital 966 motion under the ice bottom (see Wadhaus, 1973, for an early estimate of the involved process). 967 The second impact is the scattering and reflection of wave energy. In the context of a third 968 generation wave model, this is an energy-conserving process, directionally redistributing energy 969 within the spectrum, again in a way strongly dependent on the ice characteristics and the 970 wavelength. See Masson and LeBlond (1989) for an early approach to this aspect of the interaction. 971 The third impact is the modification of the phase speed and group velocity consequent to the strain 972 in the ice, hence its resistance to the motion induced by waves. This produces effects analogous to 973 refraction and shoaling (respectively) by bathymetry. The fourth effect is the modification of open 974 water source functions such as the wind input to waves, which is of course dramatically reduced 975 under rigid ice cover or even under grease ice that changes the microstructure of the sea surface. 976 These four effects can be more or less important for differing types of ice cover, and of course the 977 primary challenge today for modelling waves in ice is that the modeller typically has very little 978 information about ice cover, e.g. perhaps only the concentration is known, and possibly not well, 979 since it may be based on a satellite observation that is a few days old. Moreover, the ice conditions 980 may be dynamically linked to the waves themselves, suggesting a complex two-way coupled 981 nonlinear problem (Collins et al., 2015). Lastly, some of these issues, such as the reduction of wind 982 input by pliable ice cover, has hardly been given any attention by experimentalists, and so the 983 modeler can only make an educated guess of this effect.

\subsection{5 - Input fields}

986 This section provides an overview of the various input fields and the related consequences for modelling waves in coastal and semi-enclosed areas. On ocean scale wind is the most crucial input 988 field for large-scale wave models, as the source of energy and momentum of surface gravity waves. 989 Ocean currents can also produce significant impacts on the wave field in areas like the Gulf Stream 990 or Agulhas current (Holthuijsen and Tolman, 1991). In coastal areas and semi-enclosed seas 
991 additional effects and external variables start to play a role. As discussed is Section 2.1 the

992 specification of proper wind fields in such areas is a difficult task as the surrounding land masses 993 may cause strong temporal and spatial variations in wind speed and direction. In general wind 994 blowing offshore increases with fetch and neglecting these variations can degrade model 995 performance. In some areas strong wind jets may locally cause severe wind seas. Examples are the 996 bora winds in the Adriatic Sea (Cavaleri and Bertotti, 2004), mistral winds in the western 997 Mediterranean, strong wind jets near the Catalan coast as reported by Pallares et al. (2014), Tokar 998 Gap winds in the Red Sea (Langodan et al. 2014), and the Tehuanos near the gap of Tehuantepec in 999 Mexico (Steenburgh et al., 1998).

1000 In coastal areas astronomical and wind effects may generate currents and water level variations. 1001 These currents can be very strong in narrow straits or in tidal inlets requiring locally high resolution 1002 grids (e.g. Westhuysen et al., 2012; Ardhuin et al., 2012). Currents also affect the kinematics of the 1003 waves causing refraction and focussing of waves. In strong opposing current wave blocking may 1004 lead to enhanced dissipation. Waves travelling on a current also change the apparent wind speed. In 1005 addition, temporal variations of water level and bottom level may affect the propagation of waves 1006 resulting in tide-induced modulations of wave height and wave period measures (e.g., Tolman, 1007 1991). Current and water levels need to be computed by dedicated flow models, driven by 1008 astronomical constituents and by wind. In many applications they are applied as a stand-alone 1009 model and their results are fed into the wave model. In many circumstances, however, waves will 1010 also affect the coastal currents and water levels which require a two-way coupled wave-flow 1011 modelling approach., see e.g. Dietrich at al. (2011) for the Gulf of Mexico, Roland et al. (2012), and 1012 Brown and Wolf (2009) for the Irish Sea (more on this in Chapter 3). Especially in the relatively 1013 shallow coastal zone an accurate prediction of water levels and currents is important for depth1014 limited wave breaking and the propagation of swell waves.

1015 The spatial distribution of bottom characteristics is required to scale dissipation processes due to 1016 bottom friction, mud layers and vegetation. In many coastal areas these fields are either sparsely 1017 known or they vary in time (on time scales larger than those associated with movable beds, see the 1018 Kerala case in Samiksha, 2015, and Shinu et al., 2017). Acquisition of accurate bathymetry is, in 1019 fact, often a primary challenge for operational nearshore modelling, especially for military 1020 applications where the region in question is typically not easily accessible. Further, within what 1021 engineers call the "depth of closure", sediment transport is active, and beach profiles can be 1022 strongly affected by the activity of storms (or the lack thereof). During storms, bathymetries may be 1023 much different than they were when surveyed, making it difficult to perform an accurate hindcast. 1024 This implies that bottom surveys should be carried out regularly to retain accurate bathymetries. A 
1025 practical problem is that information on the spatial variation of bottom characteristics, like grain 1026 size, is often difficult to obtain. A common practice, then, is to neglect the spatial variation of 1027 bottom characteristics and to select a constant value for the whole computational domain, despite 1028 the well-established heterogeneity of the seafloor (e.g. Holland and Elmore; 2008).

1029 In semi-enclosed seas wave boundary conditions need to be specified, most often obtained from a 1030 larger-scale ocean wave model. Specification of these conditions is especially relevant for the 1031 lower-frequency wave components like swell waves, as these may penetrate through the whole 1032 computational domain independently of local wind conditions. In fully enclosed seas like the Black 1033 Sea, wave boundary conditions are not required as all waves are locally generated. Nesting is also 1034 required when modelling a coastal area bordering a large inner basin or an open ocean. Nesting of 1035 wave model grids may sometimes lead to consistency problem in cases where different types of 1036 wave models are nested in each other. For instance, when the SWAN model is nested into the 1037 WAVEWATCH III model, different physical packages may lead to changes in total energy and 1038 spectral shape along the grid boundary. In addition, subsequent nesting to finer grids should be 1039 carried out with resolution ratios small enough to retain the spatial variation along the grid 1040 boundaries (a resolution ratio of about 5:1 is often suitable, in our experience). Boundary forcing 1041 can affect the skill of a nest in ways that are not always obvious. For example, Rogers et al. (2007) 1042 found that while total energy may be well-predicted near the boundaries in a nest, inaccuracies in 1043 the directional distribution of its boundary forcing translate to poor prediction of total energy in the 1044 interior of the nest, since the impact of islands and bathymetry within the nest is strongly dependent 1045 on the swell directions.

1047 2.3.6 - Spatial resolution

1048 There is an interplay between the resolution of the computational grid of the wave model and the 1049 resolution of the input fields. If all input fields (including bathymetry) are coarsely defined, there 1050 will often be limited benefit for a highly resolved computational grid. Conversely, a coarse wave 1051 model cannot benefit from highly resolved forcing. Spatial resolution is also connected to the 1052 accuracy with which the underlying equations are solved. As a general rule the spatial resolution 1053 should be sufficiently fine where accurate results are required. An optimal way to achieve this is to 1054 apply either nested telescoping grids with increasing resolution or to apply unstructured grids (e.g. 1055 Benoit et al. 1996, Roland, 2008, Zijlema, 2010, Huchet et al., 2015; see also Section 2.4). Higher 1056 spatial resolutions are required where high gradients in wave conditions occur. As this is difficult to 1057 determine a priori, a commonly applied approach is to link the density of grid points to depth and 1058 gradients in bathymetry. A particular example is the cited archipelago sea located in the southern 
1059 part of the Bothnian Sea (Tuomi et al., 2012; Björkvist et al., 2016). One of the problems is 1060 associated with identifying the start of the fetch. In cases where the wave model is run in 1061 combination with a flow model, also (expected) gradients in flow conditions should be taken into 1062 account. In the context of non-stationary eddies within a flow prediction, the strategy of "higher 1063 resolution where needed" requires a so-called "adaptive grid", i.e. a grid which changes with time. 1064 This type of approach, while common within other disciplines, is relatively new and has seen 1065 limited use (so far) in wave modelling. One example is provided by Popinet et al. (2010).

\section{$1067 \quad 2.3 .7$ Spectral resolution}

1068 Spectral resolution applies to both the frequency (or wavenumber) range and direction. For ocean applications a frequency range of $0.03 \mathrm{~Hz}$ to $0.6 . \mathrm{Hz}$ and a directional resolution of $10^{\circ}$ have been considered to be sufficient, but note that proper evaluation of the air-sea interactions, in particular between waves and the forcing wind, may require a higher upper frequency limit. In high-resolution coastal applications with initial wave growth starting from land, a higher upper model frequency may be required to properly resolve the spectrum as in fetch-limited situations peak frequencies close to shore are about $1 \mathrm{~Hz}$, especially for low wind speeds. Bottema and Van Vledder (2008) report an upper value of $1.9 \mathrm{~Hz}$ for hindcasting waves in inland lakes. Additionally, higher directional resolutions of, say $5^{\circ}$, may be required to accurately resolve swell propagation with a narrow directional spread.

\subsubsection{Validation}

1080 Validation is an important part of coastal wave modelling, just as with modelling of larger scales. One major challenge to such validation exercises is that, as we have discussed, the wave field tends to be relatively inhomogeneous in the nearshore, making sparse or isolated point measurements less useful. This is especially true in tidal inlets where strong gradients in both bathymetry and current field exist. In very shallow water, validation can be reliably done only when both the bottom level and the water level are accurately known. Radar data can be especially useful for providing spatial variation (e.g., Kleijweg et al., 2005), but unfortunately, such data tends to be less reliable (more noisy, etc.) than in situ data.

\subsubsection{Discussion}

1090 The requirement of providing sufficient accurate input fields has already been discussed in the 1091 context of resolution. However, it is worthwhile to discuss also in a more general sense of 1092 uncertainty. As resolution increases, the relative importance of different physics changes. A good 
1093

review is found in Battjes (1994), then reproduced in Young (1999). Ardhuin and Roland (2013) present an overview of the various challenges when predicting waves in coastal environments. They also present a hierarchy of factors affecting the overall model performance. In decreasing order of importance they note that the accuracy of forcing fields is usually the most important, i.e. wind, wave boundary conditions, currents, bathymetry and water level. Hereafter, the quality of the source term parameterisations is important. Finally, the quality of the numerical schemes is of importance. It is noted, however, that this hierarchy does not always hold as this may depend on the type of application, see also Roland and Ardhuin (2014). For instance, in case one is interested in the propagation of a pure swell system through the North Sea, then the quality of the numerical propagation scheme and geographic resolution are probably the most important. Conversely one can readily identify cases where the accuracy of the source functions is arguably the primary source of error.

A.Roland, G.P.van Vledder, W.E.Rogers, L.Cavaleri

aaronroland@gmx.de

\subsection{Numerics}

recalling that for practical solutions we live in a discrete world

Most of the discussion in previous sections has focused on the physical aspects of the problem of wave modelling in semi-enclosed seas. There is no doubt that indeed this must be our first approach in understanding the related processes to derive the equations that express the evolution in time and space. However, while the derived physics, still with all its approximations, hold for any "point" in space-time dimensions, in practical applications we aim at evaluating the related evolution only at a limited number of "points". Therefore, the governing equations need to be discretized and numerically solved. This is far from straightforward because many processes are non-linear, interdependent, and often exhibit short scales in time and space. These problems are felt more, for different reasons, in enclosed seas. This is due on one hand to the geometry of the coastlines, the presence of islands, currents and to the frequent shallow water we come across, on the other hand to the limited dimensions of the enclosed basin characterized mainly by young and active seas. In addition, ocean swells may occasionally penetrate from an open boundary (a classical example is the North Sea between U.K. and Scandinavia). These characteristic make the numerical modelling a challenging exercise. In this section we describe and discuss the various numerical approaches that have been devised and are used to provide the best solution in the various possible situations. 
2.4.1 - Framing the problem

where we outline the basic reasons for different approaches in coastal and shallow area

When efficient numerical wave modelling in enclosed and coastal seas is of concern, the problems involved stem from the variety of spatial and temporal scales we need to resolve within our simulation. We not only have to deal with the fractal geometry of our coastlines and with bathymetries littered with small scale structures. We also come across submarine and tidal channels, reefs, bars, river outlets, wetlands, marshes, vegetation and/or bed forms, which need to be properly resolved within the utilized numerical model if the related scales are of interest. The proper numerical treatment of all the involved processes, spatial and temporal scales still bears a a significant challenge in wind wave modelling. To arrive at a practical approach, balancing computational requirements and required accuracy, we need to be aware of the possible approaches and the required assumptions and shortcuts we make to reach our goal. Indeed, acting after the physics of the model has been defined, the numerical solution of the various equations needs to take into account, at a certain level, of the approximations present in our description of some of the physical processes.

In establishing the grid where to solve numerically our equations the first and most natural solution in deep water has been the regular structured grid. A drawback of this approach is that spatial gradients of the processes we deal with cannot always be properly resolved. Classical examples are explosive cyclogeneses and typhoons/hurricanes in the open ocean, and strong localised wind jets from the coast as for mistral (French Mediterranean coast) or bora (in the Adriatic Sea). So in coastal and shallow water, the main crux of a structured grid is that it relies on a fixed resolution in geographical scale. We may relax this resolution a little by employing curvilinear grids. However, this approach is computationally inefficient to resolve small scale features, since the smallest resolution radiates through the whole domain along the grid lines. In order to overcome the strangulation of a fixed discretization, a number of different approaches have been introduced in the past two decades, ranging from nesting techniques to multigrid approaches (Tolman, 2007), or quad-tree techniques (Popinet et al, 2010). However, nesting, multi-grid and quad-tree techniques efficiently relax the constraint of a structured grid only to a limited extent. An alternative way to overcome the fixed discretization length in geographical space is given by the numerical methods based on unstructured grids. Mostly based on triangles, this approach has been pioneered in wind wave modelling by Benoit et al. (1996) based on semi-lagrangian methods, whereas the first eulerian discretization on unstructured grids was done by Liau (2001) followed by many others (e.g. Sørensen et al., 2004; Roland et al., 2006; Roland, 2008, Qi et al., 2009; Zijlema, 2009a).When 
compared to structured grids, all these approaches have shown significant improvements in terms of efficiency with respect to structured grids (Hsu et al. 2005b, Zijlema, 2009a, Zijlema 2009b, Roland et al. 2005).
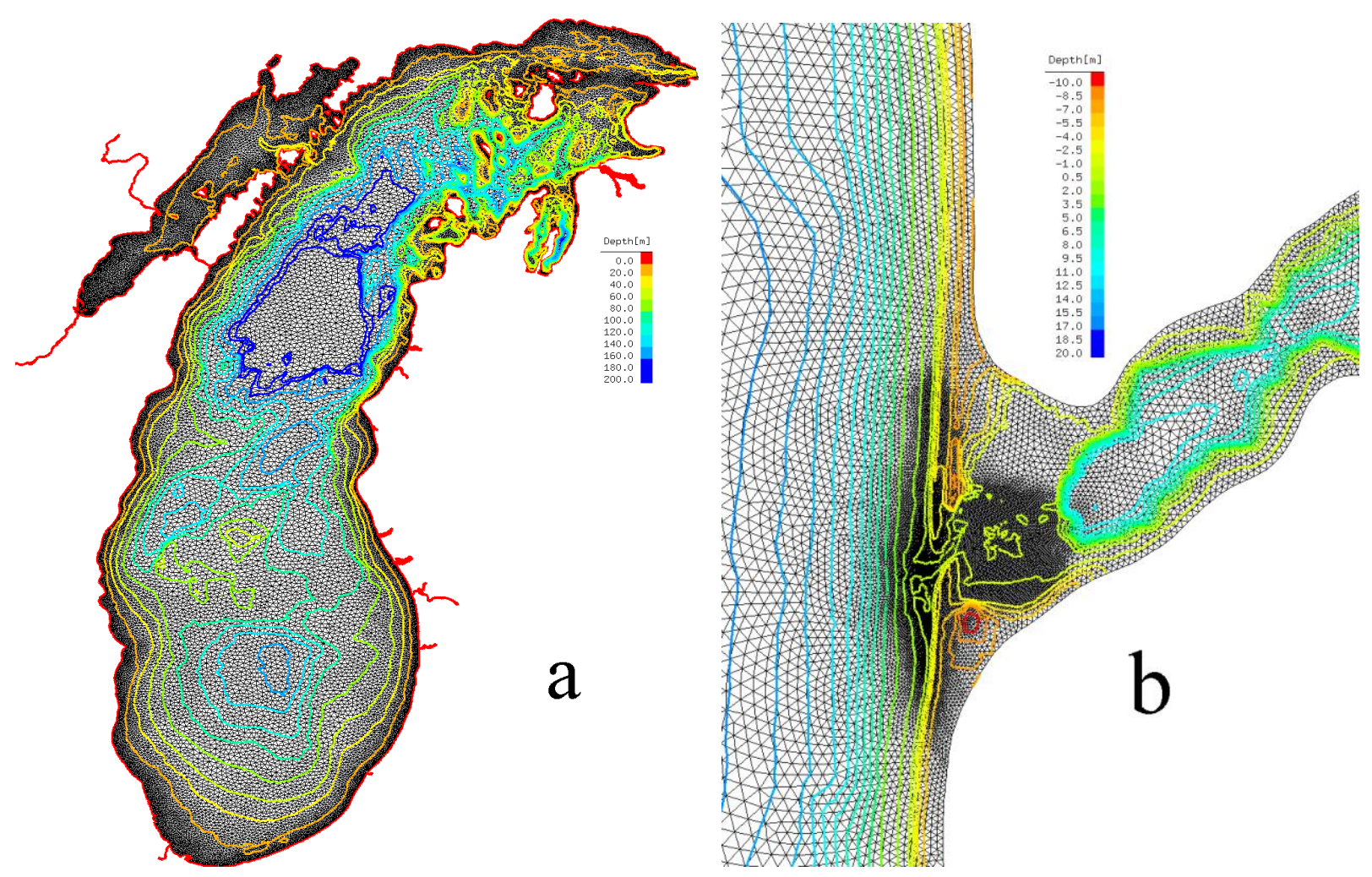

Figure 2.7 - a) Unstructured grid of Lake Michigan (Great Lakes, USA). The resolution varies from $2 \mathrm{~km}$ to $250 \mathrm{~m}$. The latitudinal extent is $500 \mathrm{~km}$. b) Numerical grid used for the coupled modelling of the Albufeira lagoon in Portugal (Dodet et al. 2013), with resolution ranging from $2 \mathrm{~km}$ offshore down to $2 \mathrm{~m}$. The colour scales indicate the water depth. Note the extremely complicated, and time variable, bathymetry and emerged sand banks. The plotted latitudinal extent is $2.5 \mathrm{~km}$.

By introducing the variability of the geographical discretization, the spatial scales in which the wave spectra change as waves approach the coast can be more flexibly adopted in spectral wave models. As illustrative examples in Figure 2.7, panel a shows an unstructured grid of Lake

1169 Michigan. The grid resolution varies from $2.5 \mathrm{~km}$ in deep water (actual operational setting at 1170 NCEP) down to $200 \mathrm{~m}$ at the coastline. The grid is generated based on the CFL (Courant et al., 1171 1928) number of the dominant wave frequency. An adaptive depth refinement is effectively limiting 1172 the total depth variations in a given triangle. In this way the characteristics of the bathymetry as 1173 well as the temporal and spatial scales of the wave physics in the near shore area can be properly 1174 resolved without increasing significantly the number of computational points as it would be the case 1175 for structured grids. The second example, panel $2.7 \mathrm{~b}$, is the extremely high resolution and real 
multiscale application of the fully coupled wave-current modelling of the Albufeira lagoon, Portugal. Here the resolution varies from the $2 \mathrm{~km}$ off-shore down to $2 \mathrm{~m}$ in the wave-current interaction region of the lagoon inlet (Dodet et al., 2013). These examples illustrate the ability to represent different spatial scales in one computational grid. The benefit of this approach is having high resolution only where required, in so doing making the number of points of the unstructured grid much lower than on the corresponding structured one.

This additional degree of freedom of having the possibility to choose the location where the equations will be solved does not come free of charge. The fees to be paid are inherently greater computational requirements per grid point (but balanced by less grid points), and the mathematical and computational complexity associated to the use of unstructured grid methods. Besides the freedom of placing the points in the domain of interest is not only a blessing: it is also a non-trivial task with respect to the grid generation process. However, present numerical methods on unstructured grids easily allow three to four orders of magnitude variation of the discretization length (the so called multiscale applications) within one numerical grid. Examples are provided, among others, by Zijlema, 2009b, Babanin et al., 2010, Dietrich et al., 2011, and Dodet et al., 2013).

Spectral wave models based on unstructured grid have become a quasi-standard solution in the last decade. These developments have also paved the way making feasible the full coupling with circulation models. This is one of the most important tools, particularly in the forecasting chain of storm surges (see Westerink et al., 2008; Roland et al., 2009; Roland, 2014, Dietrich et al., 2011; Beardsley et al., 2013; Ferrarin et al., 2013; Hope et al.; 2013, and Bertin et al., 2015), coastal hazard mitigation and for environmental studies (e.g., Ferrarin et al., 2008).

\subsection{2 - A brief outline of the available numerical approaches}

where we briefly describe the various approaches that can be used for the solution of the equations with which we describe the processes of interest

The solution of the wave action balance equation is in itself a formidable task as the equation not only has four dimensions (two spatial, two spectral) varying in time, but also because the described physics cover multiple scales of processes amply varying in time and space. The application of this kind of models has been at its beginning largely linked to operational centres, but the large computational resources that became available in the last two decades have made these models attractive to oceanographers and engineers. A thing to note is that the present scales of interest are far below the operational ones $(\sim 10-50 \mathrm{~km}$ or more) at which the models have been originally developed. With increasing computational power, finer spatial and spectral resolutions are possible 
and with more reliable measurements more demands are posed on the accuracy of the solution. The challenge therefore is to balance these requirements with the desired accuracy.

1211 Originally, and still with structured grids, downscaling in wind wave modelling became feasible 1212 with the development of nesting methods. The application of nesting, or so-called telescoping 1213 methods is at the end limited to a small region for the highest resolution grid, which may have the 1214 same constraints as any structured grid itself. As done by Tolman (2007), the idea of nesting can be 1215 generalized in the multigrid approach, where various structured grids can be combined, and also 1216 moving grids can be utilized. The latter one is a particular solution where at each time step the 1217 resolution of the structured grid can be locally increased, e.g. doubling the resolution where the 1218 gradients are above a threshold value. This approach appears useful where, like in hurricanes, the 1219 area where a higher resolution is required may and does change in time. However, although pursued 1220 for a number of years by, e.g., Popinet et al. (2010), this approach has not yet taken off, on one hand 1221 for the greater numerical complexity (e.g. book keeping), on the other hand for the continuous 1222 interpolations it requires with a consequent tendency to smooth the profile of the various variables. 1223 However, interest continues in such approaches.

1224 The benefit of the multigrid approach was the seamless two-way nesting of various grids (see the later example in Figure 2.15). However, the limitations of structured grids remain inherently the same because the highest resolution grid can only cover a limited region of interest. More recently numerical schemes have been developed that achieve higher resolution by subdividing each computation cell in a certain way (e.g., Popinet et al., 2010; Li et al., 2012). These so-called QuadTree or spherical multi cell (SMC) grid methods are much more flexible than their structured counterparts, but still the location of each node is constrained by the geometry of the grid and it does not allow a free placement. Moreover, the grid subdivision cannot be efficiently used in real multi-scale applications where in the domain of interest the geographical scale varies by several orders of magnitude.

All the present wave models, as MIKE21-SW, FVCOM-SWAVE, SWAN, ECWAM, WW3 or WWM, have introduced unstructured grids as an alternative to their structured approach. Different unstructured discretization methods in geographical space have been applied to solve the hyperbolic 1237 problem of wave propagation. These include explicit finite volume methods (e.g. Sørensen et al., 1238 2004; Qi et al., 2009), implicit finite element methods (e.g., Hsu et al., 2005a), implicit finite 1239 difference schemes (Zijlema, 2010), and implicit/explicit residual distribution schemes (Roland, 1240 2008), as given in Ricchiuto et al. (2005), These numerical schemes do relax the downscaling 1241 problem massively, but they unleash a variety of new problems, which have not been of the due 
1242 concern so far. For instance at their best they are up to second order accurate in geographical space 1243 and third order in spectral space (e.g., Roland, 2008; Zijlema, 2010). For unstructured grid methods 1244 the approximation of the time derivative is at best first order accurate. See the next sub-section for a more extensive discussion on the accuracy of the various approaches.

1246 The time marching methods used for (un)structured grids in spectral wave modelling follow two 1247 different approaches. The explicit methods are based on simple Euler time stepping or the Lax1248 Wendroff approach (Lax and Wendroff, 1960) applied within different kinds of operator splitting 1249 techniques (see, among others, Tolman, 2002a; Sørensen et al., 2004; Roland, 2008; Qi et al., 1250 2009). This approach suffers of severe time step constrains given by the CFL number. As an 1251 example, the integration time step for a shallow water $13 \mathrm{~s}$ wave in $10 \mathrm{~m}$ water depth and $10 \mathrm{~m}$ grid 1252 resolution is limited to one second. To circumvent these limitations Booij et al. (1999) and Ris et al. 1253 (1999) included the use of implicit methods on structured grids. Originally used in the SWAN 1254 model, this new approach met with immense success, and SWAN quickly became the most used 1255 model among the users community for coastal applications. The implicit methods were then 1256 implemented also on unstructured grids (Roland, 2008; Zijlema, 2010; Huchet et al., 2015).

1257 When the unconditionally stable implicit method of SWAN was presented, it was at that time the most efficient approach for solving the wave action balance equation in shallow water and it pioneered the use of this model class in shallow water, paving the way for future developments.

1260 Indeed at the time this appeared as an extremely good and the long lasting solution However, the 1261 steadily increasing computer power and the efficient parallelization now introduced in the models 1262 have largely expanded the field of application of explicit methods in unstructured grids, leading to 1263 some sort of subdivision of the respective areas of application. So the explicit approach may 1264 become the convenient solution down to $200 \mathrm{~m}$ resolution if larger regions and operational 1265 applications are of concern (e.g., Ardhuin et al., 2012, http://marc.ifremer.fr/). Conversely, implicit 1266 time stepping schemes are the convenient choice if efficient multiscale applications are of concern, 1267 especially when the variations of the grid resolution, starting from the tens of kilometres offshore, 1268 span up to four orders of magnitude down to a few metres, and if spectral wave models are coupled 1269 to shallow water phase resolving wave models.

1270 As it is obviously the case, all these discrete numerical approaches are approximations to nature. 1271 They imply errors, of which we need to be aware if we want to make a meaningful use of these no 1272 doubt very sophisticated instruments. This is the subject of the next sub-section.

$1273 \quad 2.4 .3$ - Numerical methods, their characteristics and errors 
1274 where we indicate the possible sources of error in wave modelling, how these depend on the

1275 situation and the used method, and which are the most common solutions for the daily problems

1276 Having briefly described the basic possible approaches, here we want to dig into them to quantify

1277 the errors and the limitations each of them implies.

1278 By and large the basic types of numerical errors for unstructured grid schemes are similar to those 1279 already discussed in Cavaleri (2006) and Cavaleri et al. (2007) for numerical schemes on structured 1280 grids. However, moving to shallow water, more processes become active, nonlinearities play a 1281 relevant role, scales becomes smaller, and errors may become larger.

1282 In the following discussion we will consider three basic distinctions of the possible approaches:

1283 - linear (1), nonlinear (2) [a scheme is linear in the sense that the new solution is a linear 1284 combination of the old and new ones in different grid points],

1285 - first order (1), higher order (2) [in accuracy],

1286 - explicit (1), implicit (2) [time integration method].

1287 In the above order, each approach will be classified as, e.g., $(1,2,1)$, i.e. linear, higher order, 1288 explicit. Beside its numerical characteristics, every approach can also be classified on the base of 1289 the average (situation dependent) characteristics of the obtained results. The schemes should 1290 preferably obey certain design principles that ideally fulfil the below criteria. The scheme is:

1291 (P) positive and (M) monotone - it does not produce unnatural negative results, e.g. for energy, and 1292 it does not generate new extrema in the solution,

1293 (LP) linear preserving - it guarantees higher order when the solution is smooth, and first order in 1294 the vicinity of very strong gradients,

1295 (C1) conservative - e.g., overall energy is not numerically created or destroyed along wave rays 1296 (Whitham, 1974), but it varies only on the base of physical processes,

1297 (R) robust - it is stable, and it does not depend strongly on the chosen discretization lengths in 1298 space and time as long as they make physical sense,

1299 (C2) convergent - the solution converges to a unique solution with decreasing resolution, 
1300 (E) efficient - it can be run efficiently when strong downscaling is of concern (multiscale 1301 applications) and it does not significantly depend on resolution and time step if chosen in physical 1302 reasonable ranges,

1303 (A) accurate - how close the numerical solution is to the true value of the considered variable.

1304 One key point of numerics is that, on the base of the relevant background theory, all these 1305 characteristics, in particular P, M, C and LP, are not easily obtained at once (the interested reader 1306 may refer to the various theorems by Godunov, 1959, Lax and Wendroff, 1960, and Lax-Richtmyer, 1307 1956). This will be better described in the following discussion.

1308 Starting with linear first order methods $(1,1,1)$, the classical objection is that they imply significant 1309 numerical diffusion with consequent artificial (non-physical) dissipation when adherent to coast 1310 (see Cavaleri and Sclavo, 1998). One solution is to move to linear higher order methods $(1,2,1)$ with 1311 the intent of reducing numerical diffusion. However, these linear higher order methods, no matter 1312 explicit or implicit (e.g., Hsu et al., 200a5; Qi et al., 2009; Rogers et al., 2002), do not fulfil 1313 positivity, monotonicity and linear preservation, producing in this way negative wave action and 1314 oscillations in the solution. This kind of behaviour occurs especially in regions where steep 1315 gradients in the solution are present. To treat this negative wave action, certain heuristic methods 1316 have been considered, an example being the conservative rescaling used in WWI (Tolman, 1992) 1317 and SWAN. However, this artificial redistribution of wave action in the directional space transfers 1318 some energy in other areas of the spectra, which can be seen as numerical diffusion in theta space. 1319 The magnitude of the possible negative wave action depends hereby on the gradient of the wave action in the various dimensions and the CFL number. Besides, it is clear that, albeit (but not always) at a limited extent, not all the directional conservation properties of the schemes are then 1322 fulfilled.

1323 As a consequence, this behaviour of higher order schemes imposes a constraint on the chosen time 1324 step with respect to model convergence rather than due to stability problems. Refining the mesh 1325 may alleviate these problems, but this may also increase them since it may lead to even higher 1326 gradients as it is often the case using unstructured grids. Basically unstructured grids are intended to 1327 do so by construction, and therefore linear higher order methods are hard to control.

1328 A fundamental approach to this problem is to construct nonlinear higher order schemes $(2,2, *)$ that 1329 are globally positive and flux-conservative (Godunov, 1959). The nonlinearity is essential to jump 1330 over the Godunov theorem and fulfill P, M, C1 and LP at the same time. However, implicit nonlinear higher order schemes $(2,2,2)$ are not yet available to the spectral wave modelling 
1332 community since this would involve the solution of a nonlinear equation system, which is very 1333 costly. Only non-linear explicit schemes $(2,2,1)$ have been successfully employed so far, mostly on 1334 structured grids (WW3, see Tolman, 2009), showing significant benefits when swell propagation 1335 over long distances is of concern.

1336 The conditions change drastically in coastal seas. Here the use of higher order propagation schemes 1337 can even have surprising undesired consequences.. For instance, in nature, in the lee region of not 1338 well resolved islands, refraction, diffraction and nonlinear propagation will result in wave energy 1339 transport in the shadowed regions of the islands (see, e.g., Holthuijsen et al. 2003, Liau et al. 2011, 1340 Willebrand, 1975, and Toledo et al., 2012). However, higher order numerical schemes indicate a 1341 perfect sheltering of waves, a fact not seen by first-order schemes where the implicit diffusion helps 1342 to compensate, up to a certain point, for the lack of, e.g., diffraction, nonlinear propagation, swell 1343 decay, scattering, and all the processes we have only begun to take into account in spectral wave 1344 modelling. For higher order schemes the related problems can be alleviated by explicitly adding 1345 diffusion as proposed by Booij and Holthuijsen, 1987. . Moreover, as e.g. pointed out in Cavaleri 1346 (2006), one reason for the success of first order schemes is that wave action gradients in 1347 geographical space are usually not strongly pronounced (see also Janssen, 2008).

1348 The lesson is that when we increase the order of our schemes, we need also to increase the number 1349 of physical processes we take into account, processes that are now partly mimicked by the 1350 numerical diffusion. As a matter of fact, most of the published results on unstructured grids apply 1351 first order schemes with good results even in complicated environments (e.g. Ardhuin et al. 2012), 1352 even if the numerical errors in terms of diffusion are known to be significant.

1353 Still about spatial resolution, when decreasing (i.e. improving) it is of concern, the numerical 1354 schemes should be efficient, robust and accurate even if the resolution varies over several orders of 1355 magnitude. The maximum possible time step should not depend significantly on the CFL number as 1356 it is the case for, e.g., non-monotone schemes. The scheme should also not have a significant time 1357 step dependency (robustness). The computational cost should not increase exponentially as function 1358 of unknowns and the scheme should perform efficiently also when parallelizing the program 1359 (efficiency). Moreover, the scheme should converge $(\mathrm{C} 2)$ as fast as possible to a weak (i.e. 1360 pragmatically, but not formally, correct) solution if we reduce the time step and increase the 1361 resolution (accuracy). 
1362 Another class of numerical errors, the so called splitting errors, arises in e.g. the fractional step 1363 method (Yanenko, 1971). They follow the separate numerical treatment in various dimensions of 1364 the advection and source terms (e.g. Tolman, 2002b). The splitting error becomes significant when

1366 Table 2.2 Numerical characteristics of the different solutions adopted in the various approaches.

\begin{tabular}{|l|l|l|l|l|l|l|l|l|}
\hline \multicolumn{4}{|c|}{ discretization } & \multicolumn{4}{c|}{ properties } & \multicolumn{2}{c|}{ downscaling performance } \\
\hline linear/nonlinear & $\begin{array}{l}\text { first/higher } \\
\text { order }\end{array}$ & $\begin{array}{l}\text { Time- } \\
\text { stepping } \\
\text { method }\end{array}$ & $\begin{array}{l}\text { conservative- } \\
\text { convergent }\end{array}$ & $\begin{array}{l}\text { linear } \\
\text { preserving }\end{array}$ & positive & efficiency & robustness & accuracy \\
\hline linear & first & explicit & yes & no & yes & average & high & low \\
\hline linear & first & implicit & yes & no & yes & highest & highest & lower \\
\hline linear & higher & explicit & yes & no & no & average & low & low \\
\hline linear & higher & implicit & yes & no & no & low & low & low \\
\hline non-linear & higher & explicit & yes & yes & yes & average & high & high \\
\hline non-linear & higher & implicit & yes & yes & yes & high & high & high \\
\hline
\end{tabular}

1368 the operators do not commute and the global (i.e. large scale) time step is much bigger than the one 1369 locally used for each of the sub-problems (source terms or spectral advection - see, e.g., LeVeque 1370 and Yee, 1990; Lanser and Verwer, 1999; Sportisse, 2000, and Geiser, 2012). The splitting error 1371 between source terms and geographical advection, as well as the one versus spectral advection, may 1372 become significant for large global time steps when a large number of sub-iterations are required. 1373 The optimal case would be that each of the various spaces has unity sub- or just some few sub-steps 1374 in the various dimensions. For splitting schemes, however, it can be shown that in stiff cases (i.e. 1375 cases for which a small time step is required for stability) there is a severe order reduction of the 1376 scheme. It must be noted that splitting methods that use implicit schemes for geographical 1377 advection are much more prone to splitting errors since large global time steps are possible. This 1378 leads to significant splitting errors, especially if for the purpose higher order linear schemes are used (see, e.g., Roland, 2008, Roland \&Ardhuin, 2014). Consequently, for numerical schemes which have a pronounced dependency on the integration time step it is advisable to carry out a convergence analysis by reducing the time step and increasing the resolution up to the point where

1382 the resulting difference are in the acceptable range for the specific purposes. This mainly involves 1383 splitting methods and higher order linear schemes. To summarise, in order to be able to judge the 
results, it is advisable a) when applying higher order linear schemes, to either carry out convergence studies (e.g. by refining the mesh and/or reducing the time step) or b) even to apply first order schemes, which are more predictable in how they characterize the solution.

To frame the situation, we can say that suitable nonlinear higher order schemes are not yet available for the extensive daily activity, and implicit first order methods on unstructured grids are probably at this time the best approach while having a sufficiently resolved grid/mesh, especially in applications that consider coastal and enclosed seas with multiscale characteristics. However, if new physics is to be investigated, using first order schemes it will remain difficult to properly quantify the numerical errors versus the ones arising from the investigated physical description of the processes. Here it is important to design test cases, where the physical phenomena of interest are isolated and the effects of numerics significantly reduced, something that can only be achieved by higher order schemes or high spatial resolution.

\subsection{4 - Limiter effects}

where, after describing the possible errors, we indicate the most common used solutions

Notwithstanding the problems we have briefly described in the previous sub-sections, we need 1) to produce results, 2) to be aware of the possible errors, 3) to devise methods to limit these errors as much as possible. Knowing by theory and experience where the problems may arise, we can use some pragmatic approaches to limit the undesired consequences. This leads us to the subject of limiters.

Limiters are desired in operational wave modelling for the sake of robustness and efficiency and act as a safeguard in many situations. The limiters are designed to a) limit instabilities arising in stiff systems, e.g. related to the nonlinear four-wave interactions if operating with a large time step, b) limit spectral space advection velocities (viz., $c_{\theta}$ and $c_{\sigma}$ ) if they become too high, and c) limit the maximum wave height when depth induced wave breaking is of concern (see the classical paper by Battjes and Janssen, 1978). This happens, e.g., when the integration time step is chosen to be too large for the problem of interest, or if the advection velocities, e.g. due to steep and under-resolved bathymetry, are too large. (see Komen et al., 1994; Booij et al. 1999; Hersbach and Janssen, 1999; Tolman, 2002b; Monbaliu et al., 2000; Hargreaves and Annan, 2000; Zijlema and Van der Westhuysen, 2005; Tolman, 2009; Roland and Ardhuin, 2014). In the c) case above, the wave breaking limiter acts as a safeguard with respect to the physical value of wave heights in shallow waters. In practice we need to be sure that, based on numerical and discretization errors or the 
1416 wrong use of the physical formulation, the wave model does not produce obviously unphysical 1417 results (e.g., wave heights far greater than the water depth or other non-physical artefacts). All 1418 numerical forecasting methods rely on the above summarized limiters as a pragmatic safeguard.

1419 The problem with limiters is that, once embedded in the program, they may appear and virtually 1420 become part of its physics. However, ideally these limiters should not have a big influence on the 1421 solution. In this respect we should not forget that most of these limiters have been originally 1422 designed for global wave forecast and for operational needs (see, e.g., Monbaliu et al., 2000). In 1423 most of the cases they appear in their original form in the present models. For instance Hersbach 1424 and Janssen (1999) and also Tolman (1992) have shown that, especially in the initial growth stages, 1425 the results depend strongly on the integration time step. This is of special significance for enclosed 1426 basins where the typical conditions of a (fetch-limited) developing wind sea with high spatial and 1427 temporal gradients may impose restrictions to the integration time step.

1428 Apart from playing with the resolution, to circumvent this problem we could also redesign the 1429 numerical methods (Tolman, 2009) or reformulate the wave action limiter with the aim to reduce 1430 their impact on the solution. The alternative limiter suggested by Hersbach and Janssen (1999) 1431 reduces significantly the time step dependency on the solution showing very good results in 1432 operational forecasting, but it has also shown oscillations for small time steps (Monbaliu et al., 1433 2000) and has not yet been significantly tested in shallow waters. Actually, the Phillips limiter by 1434 Komen et al. (1994) acting on the rate of change in the spectral tail is presently used in most wave 1435 models, also when applied in shallow water modelling (Booij et al., 1999). In these circumstances 1436 the integration time step is the crucial parameter, especially if transient solutions are of concern. 1437 Further research is needed to design a limiter formulation for implicit schemes in shallow waters, a 1438 (wave action) limiter which does not introduce such a significant time step dependency in the 1439 solution and makes in this way more efficient modelling possible, both in terms of computer time 1440 and accuracy.

1441 In many approaches limiters have been applied also to the propagation velocities in spectral space 1442 (refraction and frequency shifting) in terms of bottom slope, spatial resolution or other parameters. 1443 Some models allow the user to limit the refraction for under-resolved bathymetries. This is true for 1444 most of the models, either acting on the code (WAM, WW3 or WWM) or on the SWAN input file. 1445 The consequences depend on the situation and require attention by the user (see later). Another 1446 compromise solution is to further reduce the time step in spectral space, put a maximum value to the 1447 bottom slope in each grid step, in so doing effectively placing a limit to the refraction term $c_{\theta}$ and 1448 spreading the change of depth on a higher number of steps. In this respect it is interesting to note 
1449 that, in contrast to the action density limiter, the limiters on spectral advection velocities are not 1450 required for model stability (the overall energy, i.e. $H_{s}$, is not varied), but for efficiency or accuracy 1451 reasons (we do not want to change too much the distribution of energy in the spectrum). However, 1452 at the end of the day, whichever the accuracy we can use in our action, the relevant point is that the 1453 application of these limiters may have significant effects on the results. Therefore their implications 1454 should be investigated in terms of convergence studies (e.g., Roland and Ardhuin, 2014). It is 1455 highly advisable to explore the convergence of our model and, e.g. depending on the grid 1456 resolution, 1) how necessary is indeed a limiter, 2) if its use is prone to substantial local errors.

2.4.5 - Summary and outlook

where we summarize where we are today, but then look forward to the future problems and numerical possibilities

1462 The discrete description of the natural world we are forced to use in practical applications requires 1463 its fee, and we have seen this is paid in terms of both computer time and accuracy, the latter 1464 depending again on resolution and errors of the results. All this is enhanced in the enclosed seas: the 1465 limited dimensions of the basins imply shorter waves, frequently under wind generated conditions. 1466 We also have complicated geometry of the coasts, shallow water conditions, steep gradients both in, 1467 e.g., the bathymetry and in the values of the variables (significant wave height, periods, directions, 1468 spectral distribution) we care about and need to know.

1469 As expected, this has stimulated the development of a variety of methods (linear and nonlinear, first 1470 or higher order, explicit or implicit, regular and unstructured grids) that time and experience have 1471 helped to frame with respect to their characteristics and applicability in the different environments 1472 and situations.

1473 Talking about the last one of the just mentioned characterizations, there is no doubt that 1474 unstructured grids have gained much ground in applications in the inner seas and coastal water. 1475 When approaching a complicated coastline and a highly varying bathymetry, a flexible and 1476 adaptable grid (although not yet dynamically varying) is the wanted and obvious solution. Of course 1477 the same situations that lead to this choice imply more complications in the numerical solution of 1478 the (partial differential) equations with which we describe the physics we care about. This may 1479 range from the "simple" problem of wind waves to the complex ones of storm surges or 1480 environmental studies dealing with sediment transport and water quality. 
1481 The choice between explicit and implicit methods seems to depend on the scale of the area of 1482 interest and the situation. Implicit schemes offer advantages and are the best choice if at the coast 1483 we go at resolution of the order of $\sim 10 \mathrm{~m}$ or less. Indeed examples of implementations in WW3, 1484 WWM-III and WAM, besides the obvious SWAN, exist. At a higher scale, if computer power is 1485 available and thanks to parallelization, the explicit approach has still its ground up to a certain 1486 extent, say $200 \mathrm{~m}$ resolution, provided points in higher depth, like e.g. shipping channels, are not 1487 considered. In the latter case one "bad point" can destroy the whole performance of the explicit 1488 scheme, which means that much more time in grid generation needs to be spent in order to have an 1489 efficient mesh in contrast to implicit schemes where this would not matter in terms of performance. 1490 The situation is flexible to a certain extent.

1491 Much effort has been put in developing explicit nonlinear higher order schemes, ideally to avoid 1492 some of the apparent limitations present in linear first and higher order ones. However, the results 1493 are debatable, and there are indications that first order schemes are still a valuable solution. This is 1494 also connected to a lack of knowledge and to the discrete use of the physics of the many processes 1495 that appear with a potentially relevant role in shallow water. We compromise this with the use of 1496 numerical limiters to avoid either unphysical values of physical parameters, or excessive fluxes 1497 either in space or spectral terms. Apart from the actual physical or flux value, from the numerical 1498 point of view these limiters may imply problems in the convergence to the right solution. Therefore 1499 convergence studies are highly recommended to establish a priori if and when a limiter is needed, 1500 and, if so, its more suitable value. Future developments should be aimed at designing schemes in 1501 such a way that the effects of heuristic measures on the solutions vanish or are strongly reduced, in 1502 so doing reducing also the numerical uncertainty in wave prediction in shallow water conditions. 1503 This will imply some basic steps in the extension of the first order schemes towards higher orders, 1504 particularly nonlinear implicit ones.

1505 With this in a way we are still on the "traditional" ground of the energy or action balance equation. 1506 However, the new frontier opened by pushing, e.g., the models closer and closer to coast, in 1507 shallower and shallower water, practically in the breakers zone, implies extremely strong spatial 1508 and temporal gradients, hence new physics and new interactions to be considered. If, but still we do 1509 not know how, we want to model this area in an effective way, obviously new approaches, 1510 equations, numerical methods need to be developed.

1511 More in general, we should never assume that the ground we firmly stand on and that has been there 1512 for a while will be our permanent solution. New openings, not only numerical, but certainly related 1513 to numerics, are presently explored. Already in 1975 Willebrand had worked on nonlinear 
1514 scattering relationship, which imply the extension of the equations to inhomogeneous media. 1515 Toledo et al. (2012) have explored the extension of the energy balance equation to higher order 1516 bottom slopes and shear currents, an extension that results in a nonlinear, amplitude dependent 1517 equation and a new definition of the conserved variables since wave action is not conserved 1518 anymore under such conditions. In terms of efficiency the extension to wave models based on these 1519 innovative approaches will imply a significant breakdown of our forecasting range, demanding a 1520 complete revision of our numerical approaches before operational applications are even thinkable.

1521 Further problems, still related to coastal or, e.g., harbour features, concern wave interference and 1522 diffraction that cannot be dealt with by a homogeneous and Gaussian statistics. Recently Smit and 1523 Janssen (2013) and Smit et al. (2015) have developed a generalized evolution equation for the 1524 transport of the complete second order wave statistics of the surface elevation, including cross1525 correlation. Specifically, this equation generalizes the action balance equation by including the evolution of the cross-correlation terms. This approach will improve the physical basis of e.g. computing the sheltering and spreading of waves behind an island, as discussed in section 2.4.3.

1528 We have repetitively mentioned the basic problem of representing in a discrete way the natural continuum. In meteorological modelling the spectral approach solves this issue by representing the fields as two-dimensional Fourier series (see, e.g., the Integrated Forecast System, IFS, of the European Centre for Medium-Range Weather Forecasts, Reading, U.K., www.ecmwf.int). Yildirim and Karniadakis (2012) did so in wave modelling using a spectral approach in spectral space. More recently, Adam et al. (2016) introduced the so-called adaptive Haar wavelets, which is even more promising. How effective this approaches can be in strongly non-periodic fields is to be seen.

Possible future developments will be further commented upon in the general look to the future in Chapter 5.

Robert.E.Jensen@erdc.dren.mil

\section{5 - Data availability}

where, contrarily to the common attitude of wave modellers, we make an extensive critical analysis of the wave measuring instruments, hence of the accuracy we can expect on the data we use for the validation of our modelling work. We also stress the need to go further than the usual integrated parameters to have a detailed and more meaningful validation of the model results. 
1548 Availability of the sea truth, of wave conditions in particular, is a key element for the validation, 1549 and consequent improvement, of model results. In this section we provide an overview of the 1550 commonly used technologies to provide those data, highlighting their advantages and limitations, 1551 and what to expect for the immediate future. Unavoidably the various arguments will not be valid 1552 only for coastal or shallow waters, or for inner seas. However, we also mention specific problems 1553 generally typical of this environment.

\subsection{1 - Generals}

where we outline the importance of measured data and their accuracy

1558 We have been directly measuring wind-generated surface gravity waves (henceforth 'waves') for over 60 years and continue to operate and maintain an array of measurement platforms around the world to this day. Wave measurements in the context of enclosed, semi-enclosed bodies of water or the coastal domain have and continue to be a necessity in all aspects of wind-generated surface gravity wave studies. Wave measurements have been, are and will continue to be used in evaluations, model testing/improvements, source-term specification, climate trend analyses.

1565 Our roots in the development of wind wave growth rate expressions are based on field measurements in enclosed bodies of water such as Lake Okeechobee, Florida (Bretschneider, 1952), Lake Ontario, US-Canada (Donelan et al., 1985), Lake St Clair, US-Canada (Donelan et al., 1992), Lake George, Australia (Young and Verhagen, 1996a and 1996b). Even JONSWAP (Joint North Sea Wave Project, Hasselmann et al., 1973) was conducted in what would be classified as a coastal region in the North Sea. The Surface Dynamics Experiment (SWADE, Weller et al., 1991) and the

1571 Shoaling Waves Experiment (SHOWEX, Ardhuin et al., 2007) were conducted along the US 1572 Atlantic Ocean operating on the continental shelf, investigating wind-wave growth, and transformation processes. These field campaigns highlight the need of accurate data in coastal and enclosed seas. Before discussing the characteristics of the main instruments in use, we make a brief panorama of the different approaches.

Waves have been measured using various measurement platforms from point-source sites, such as surface buoys, bottom mounted systems (pressure), acoustic profilers, to fixed systems as in the case of continuous wire gauges, step resistance, to downward looking laser. Each system has a welldefined range of application, placed at a site with the general purpose of monitoring local conditions. Problems occur of course. As a first hint we mention that wave measurement systems 
1582 used in enclosed, semi-enclosed water bodies and coastal waters have to consider the frequency 1583 range of the wave climate, the water depth where the devices are placed that can vary based on 1584 tides, surges, seiching of the free surface as well as changes in the bottom, and the breaker zone 1585 where the free surface cannot be distinguished any more. For bottom mounted gauges, the water 1586 1587 1588 1589 depth acts as a low-pass filter, reducing the ability to measure high frequency wave energy. Wavecurrent interaction effects from the diurnal oscillation of tides, wind generated, or in shallow water wave-induced ones can and will modulate the measurements and produce erroneous results. It is clear that we need a thorough look at what wave measurements can really provide. Most of all, wave modellers need to realize that, contrary to the use often done, measured data are not that perfect piece of information we wou8ld like to have. Rather, they have statistical and intrinsic errors that we elucidate further in this section where we briefly describe the main methodologies used to measure waves and the related implications for accuracy.

\subsection{2- Types of measurements}

where we mention the main systems for measuring waves and their respective characteristics

There are two primary types of instruments to measure waves: wide scope measurements, using remote sensing including satellites, aircraft equipped or land-based systems, and point-source measurements i.e. focusing attention on a very limited area, most of the time a single point. Both have strengths and weaknesses. Satellite systems provide large-scale, repetitive spatial coverage, but with discontinuities in time. Land based radar systems cover with continuity a certain area, but they lack details and accuracy. Applications to land-based radar systems measuring waves have been questioned and require further testing and evaluation of historical ones. Point measurements provide data continuity, but of course limited at a single location.

\subsubsection{1- Remote sensing}

\section{Altimeters}

Numerical Weather Prediction Centers (NWP) as ECMWF, NOAA/NCEP, UKMO, and FNMOC forecast wave conditions for the entire ocean covering the world. Prior to launch of the short living SEASAT (1978) and GEOSAT (1985) and the consequent availability of altimeter wave height estimates, assessing the quality of large scale model results was impossible to achieve. Satellite based remote sensing, using altimeters such as those on-board TOPEX/Poseidon, Jason-1, Jason-2, Jason-3, ERS-1, ERS-2, ENVISAT and Sentinel-3 and using synthetic aperture radar (SAR) such as 
1616 those on-board ERS-1, ERS-2, ENVISAT and Sentinel-1, has provided and provides useful data in 1617 remote locations of the world's ocean basins up to a few tens of kilometres off the coast (but see 1618 the recent advances with Sentinel-1, e.g. by Ardhuin et al., 2015). NWP's routinely use these results 1619 to evaluate the wave forecasting performance (Romeiser, 1993; Janssen et al., 1997; Bidlot et al., 1620 1997; Janssen et al., 2003; Li and Holt, 2009), or, via the assimilation into a first-guess wave fields, 1621 to improve the wave forecast quality (see Abdalla and Janssen, 2017, for an extensive discussion on 1622 the subject). Note that using the same data for both the purposes has not much significance.

1623 Despite the resounding success of using altimeter data for assimilation and validation purposes (but 1624 see Chapter 4 in the former respect), there are in fact degrees of uncertainty in these records. The 1625 statistical uncertainty of sampling a random sea is generally reduced by the ample (a few kilometre 1626 diameter) sample area at each radar shot (available at a rate of 20 per second) and by providing one 1627 a second data averaged over the 20 shots. However, thinking especially of coastal and inner waters, 1628 if strong spatial gradients are present, it is clear that providing one datum every seven kilometres 1629 has a strong tendency to smooth the field.

1631 In general, specific altimeter instruments can be quite different from each other. Indeed measurements by various altimeters are not consistent, but they may differ by few per cents. Besides the calibration of these instruments is usually done in the 2 to 4-m wave height range, while the ones on the lower and higher ranges can be, and usually are, different. Finally most of these calibrations have been done based on measurements by open ocean buoys (see Figure 2.8, but also the later discussion about buoy data) where the usual wave conditions are different from the enclosed seas, and this affects the calibration.

The mentioned difficulties close to coast are connected to the discontinuity felt in passing from land 1640 to sea and vice versa. The European Space Agency funded COASTALT, a study on the 1641 development of altimetry in the coastal zone for ENVISAT, which started in 2008 and has reached 1642 completion a few years ago (COASTALT 2011; see http://www.coastalaltimetry.org/ ). A similar 1643 study PISTACH (see

1644 https://www.aviso.altimetry.fr/fileadmin/documents/data/tools/hdbk_Pistach.pdf) was

1645 commissioned by CNES. Pilot studies have been undertaken with the goal of developing algorithms 1646 that can adequately and accurately recover altimeter information in coastal waters (e.g. Cipollini et 1647 al., 2009; Vignudelli et al., 2012). 
1649 A strong effort has been performed to improve data recovery and accuracy in coastal areas with the 1650 launch of the first and third ones of the Sentinel satellite array. While the first results suggest some 1651 optimism, calls have been issued by the European Union for projects assessing the quality of, and 1652 finding optimal ways to interpret, raw altimeter data. In particular the attempt to use the data from 1653 each single radar shot could provide data closer to coast, but at the price of a much larger 1654 uncertainty (up to $0.5 \mathrm{~m}$ error). See in this respect the well summarizing ppt available at 1655 https://owncloud.ve.ismar.cnr.it/owncloud/index.php/s/ncbmkTWQjKMIulr.

1657 Radars

1658 Given that waves, as a geometric feature of the sea surface, reflect radar waves, it is only natural to use this instrument to measure waves. Remote sensing systems have also been used on board fixed winged aircrafts. In particular, airborne scanning LIDAR systems (Hwang et al. 2000), Scanning

1661 Radar Altimeter (Walsh et al. 2002), airborne radar RESSAC (an a C-band radar with a scanning 1662 beam antenna, Pettersson et al., 2003) have been used for specific short-term missions mapping the 1663 spatial variability in the wave climate over short time periods, particularly in coastal areas. Hwang 1664 et al. (2000) investigated quasi-steady state wave fields under active generation, and a decaying 1665 wave field following slacking winds. The uses of these data, as they generally occur in a near coastal region, are specific to either a large-scale field experiment or a unique meteorological situation, and are of high directional resolution.

1668

Closer to surface, X-band marine radars have been used transmitting and receiving pulses of microwaves at grazing incidence. The radar pulses interact with the centimetre-scale sea surface roughness through Bragg scattering. 3D backscatter data are processed with well-established 1672 Fourier Transform-based technique to retrieve directional wave spectra. The processing involves a 1673 series of filters and the application of an empirical Modulation Transfer Function to account for the 1674 radar image formation nonlinear mechanisms. Radar coverage of the sea surface is up to few km 1675 from the antenna, even though the backscatter signal decays super-linearly with the range and has a 1676 strong dependence on the azimuth. From radar data, the surface current (an extra information) is 1677 determined by means of a least-squares regression method that exploits the dispersion relation for gravity waves. While the X-band signal needs the centimetric waves, hence some wind, to be reflected, the HF radar, mainly used to measured currents, has the capability to interact directly with the wavelengths (10 to $200 \mathrm{~m}$ ) we mostly care about. Note that HF radar provide only direction-oflooking information, hence they need either two separate sources or to look, progressively or with multiple antennas, in different directions. In the very near-shore HF and X-Band radar systems have 
1683 been used to map the spatial and temporal variability for a specified patch of the free surface. 1684 These systems (e.g. CODAR, OSCR, WERA, and Pisces, see Wyatt et al., 2009) continue to 1685 operate at various coastal locations, however work continues to better interpret and improve their 1686 estimates of wave characteristics (see, among others, Prandle and Wyatt, 1999, and Wyatt et al., 1687 2003). These systems have also been plagued with operational constraints (dependent on individual 1688 system applications, Wyatt et al., 2003, 2005, 2009), especially in a coastal environment.

1690 Stereo

1691 Stereo imaging systems allow the 3D sea surface to be measured using digital images acquired from 1692 (at least) two distinct points of view. For typical applications, stereo imaging captures short- to mid1693 size wavelengths, in the range: $0.2-80 \mathrm{~m}$, with high frequency resolution. The accuracy of the 1694 observations is of few centimetres along the 3D axes. The pre-processing involves the calibration of 1695 camera parameters and the determination of the pose between the stereo cameras. Then, the 1696 coordinates of 3D points of the sea surface are recovered via triangulation of the corresponding 1697 pixels. Stereo wave imaging was proved to provide accurate wave fields for investigating different 1698 aspects of the oceanic waves, such as extreme (freak) waves, the shape of the directional spectrum, 1699 the phase speed of the largest waves. Benetazzo et al. (2015) provide an extensive view of this 1700 powerful system that no doubt will become more popular in the future. Figure 2.8 provides an 1701 enlightening view (one image from a 20 minute record) taken from the ISMAR oceanographic 1702 tower (see Figure 2.3 for its position). 


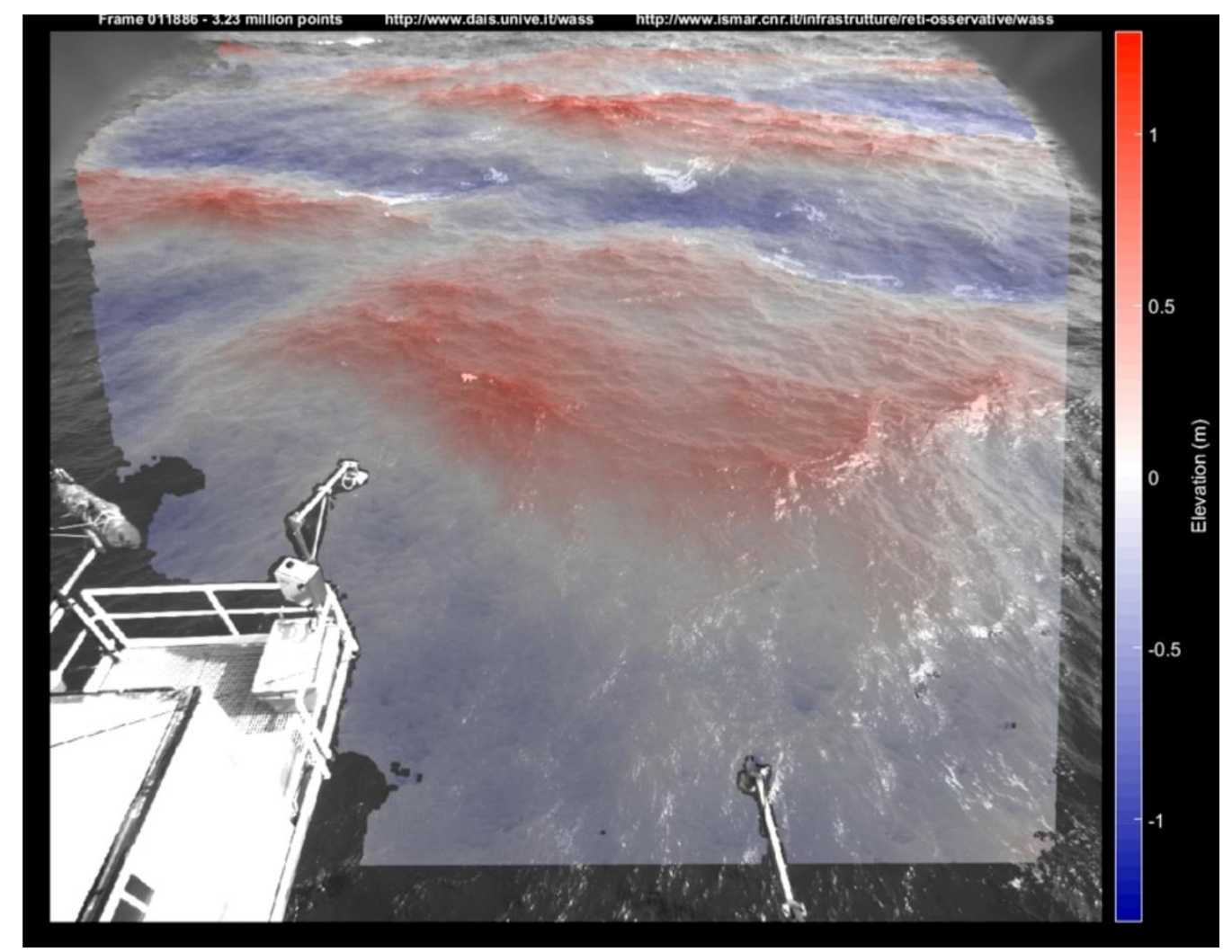

1705 Figure 2.8 - 3D field of wind waves as measured by stereo system at the ISMAR oceanographic 1706 tower. See Figure 2.3 for its position and Benetazzo et al. (2015) for the description of the system.

\subsubsection{2- Point-source measurements}

Point-source measurements have been operationally used to measure the ocean surface for the past six decades (e.g., Tucker, 1991). Most of these gauges were operated in the coastal domain. Wire 1712 capacitance and resistance gauges both effectively use depth of submergence in water to complete a 1713 circuit. Originally these gauges were affixed to piers (Thompson, 1977) and offshore platforms 1714 (e.g., Hamilton, 1972; Cavaleri and Zecchetto, 1987; Cavaleri, 2000), but they have also been 1715 modified for their use on vessels (Drennan et al., 1994) and integrated into a spar surface buoy 1716 (Graber et al., 2000). Downward looking laser altimeters (Magnusson and Donelan, 2013) are used 1717 as the baseline for an intra-measurement evaluation in the North Sea (Allender et al, 1989), and 1718 have also been affixed to a moving research vessel (Donelan et al., 2005). Nadir-looking radars are 1719 another popular solution on rigs to measure, as with laser, the mean sea level and the waving 1720 surface. The combination of three, often four, instruments provides directional information. Three 1721 transducers provide, for directional distribution, the same amount of information that we will soon 1722 discuss in buoys. 
1724 The second class of devices used again in the coastal area, are bottom mounted sensors. Bottom 1725 mounted sensors fall into two categories: pressure transducers and upward looking acoustic current 1726 profilers. Measuring waves with pressure transducers have been operationally used for nearly six 1727 decades (Bishop and Donelan, 1987; Pomaro et al., 2017). The ease of deployment, relative cost 1728 and survivability make these systems highly popular. Apart from the problems related to fouling, 1729 usually more intense in coastal waters and enclosed seas, one of the impediments using a pressure 1730 transducer are the assumptions governing the relationship to translate a pressure response to the 1731 corresponding free surface oscillation. The reason is the amplification factor from the submerged 1732 measurement point to the surface that increases pretty rapidly with increasing frequency (the 1733 inverse of the attenuation with depth). For example, in $8 \mathrm{~m}$ water depth the amplification ranges 1734 from about 1.05 for $0.054 \mathrm{~Hz}$ signals to 13.1 for $0.318 \mathrm{~Hz}$. Any noise in the pressure response will 1735 be amplified according to the frequency dependent factor. It is essential to minimize the noise or 1736 the wave estimates will be heavily contaminated.

1738 Currents, nonlinearities, spectral analysis versus wave-by-wave approaches do affect the estimates 1739 in significant wave heights. Bishop and Donelan (1987) summarized the two principal approaches 1740 to prevent contamination of the wave signal by noise. The first would be to subtract an assumed 1741 noise level from the pressure spectrum prior to translating it to a surface displacement spectrum. 1742 The second would be to truncate the pressure spectrum where noise dominates. Smith (2002) 1743 summarized the techniques to compensate pressure responses under an active current. He showed 1744 that in a coastal inlet neglecting currents can lead to order-one errors in wave heights. Cavaleri 1745 (1980) showed how to avoid the dynamical effects of current and wave motion measuring only the 1746 signal due to wave pressure. Herbers et al. (2000) built an array of bottom mounted pressure sensors 1747 in water depths ranging from 12 to $87 \mathrm{~m}$ studying the transformation of swell energy (0.07 to 0.1 $1748 \mathrm{~Hz}$ ). In all these cases the investigators relied on specific analysis procedures to remove noise 1749 and/or compensate for currents and nonlinear effects.

1751 With respect to the classical three sensors for directional information, more is possible with more 1752 dense arrays. A multiple pressure array (two six-pressure arrays) used in the Southern California 1753 Bight (Torrey Pines, California) was capable of resolving bi-directional wave systems at one 1754 frequency separated by only 8-deg in direction (Freilich et al., 1990). The complex cross-spectral 1755 matrix between all sensors in an array was averaged over eight frequency bands, resulting in a 1756 frequency resolution of $0.0078 \mathrm{~Hz}$. The cross spectra were then ensemble averaged over 20 1757 records, producing 320 degrees of freedom. An iterative maximum likelihood (IMLE) method 
1758 (Pawka, 1983, Oltman-Shay and Guza, 1984) was used converging on a possible true directional 1759 wave spectrum. The results produced extremely high resolution, high quality estimates in shallow 1760 water. A similar system using IMLE has been in operation at the USACE's Field Research Facility 1761 for nearly forty years (Long and Oltman-Shay, 1991). As in a single pressure gauge, the system 1762 assumes linear theory to recover the pressure response factor converting to estimates of the free 1763 surface, that are functionally related to the water depth and frequency.

1765 Upward looking acoustic current profilers (ADCP) have been used in the coastal environment to 1766 estimate wave characteristics. Herbers and Lentz (2010), among others, summarize the various systems identifying the limitations of each system in correctly interpreting the recorded data. Under severe wave conditions, in a limited depth coastal environment breaking can be frequent and the presence of large air bubbles just below the surface can interfere with the acoustic measurement of the surface. It is therefore convenient to shift in this case to pressure measurements, as done for instance at the ISMAR oceanographic tower (see Figure 2.3 for its position). Working in a low wave energy environment, dominated by long-period swells (Southern California), creates finite bounds to assess the reliability of ADCP wave estimates. It is then customary to define a simple parametric estimation technique capable of providing a robust estimate of the gross directional wave properties even when the quality of the data is marginal.

What just given is a short summary of the main instruments used to measure waves. We still miss what is probably the most popular one, i.e. buoys. Taking advantage of the extensive experience available with this instrument, we will discuss extensively the related problems as a detailed example of the ones that affect all wave measurements.

\subsubsection{3 - Buoys}

1784 Wave measurements across the world's oceans have been increasing, and in general the vast 1785 majority of these assets are surface wave buoys (see Figure 2.9 for a distribution). Most of these 1786 sites are located within about $200 \mathrm{~km}$ of the coasts, deployed on a continental shelf, and in water 1787 depths less than 200m. This distribution (plus the fixed platforms) does not reflect all operational 1788 wave measurement sites, only the ones that transmit data directly through the Global 1789 Telecommunication System (GTS). 
1791 Differences exist in the type of wave measurement system occupying a site, and need to be 1792 considered when using a set of wave data. The world's data providers use Quality Assurance and 1793 Quality Control (QA/QC) protocols prior to release to the GTS. However, there is no universal 1794 criteria under which the data are considered as error free. QA/QC flags are also contained in the 1795 data records, but seldom interrogated by a user. And thus, if the quality of the data were 1796 independently related to the wave system there would be no need to consider the existence of errors 1797 in the measurements. Unfortunately this is not the case and there are well defined differences. It is 1798 not to say there is one wave measurement system superior to all other known platforms. It is to have documented knowledge where and when a given wave measurement does not contain the quality required for a particular problem. Valid for buoys, of course these statements hold for every measuring system.

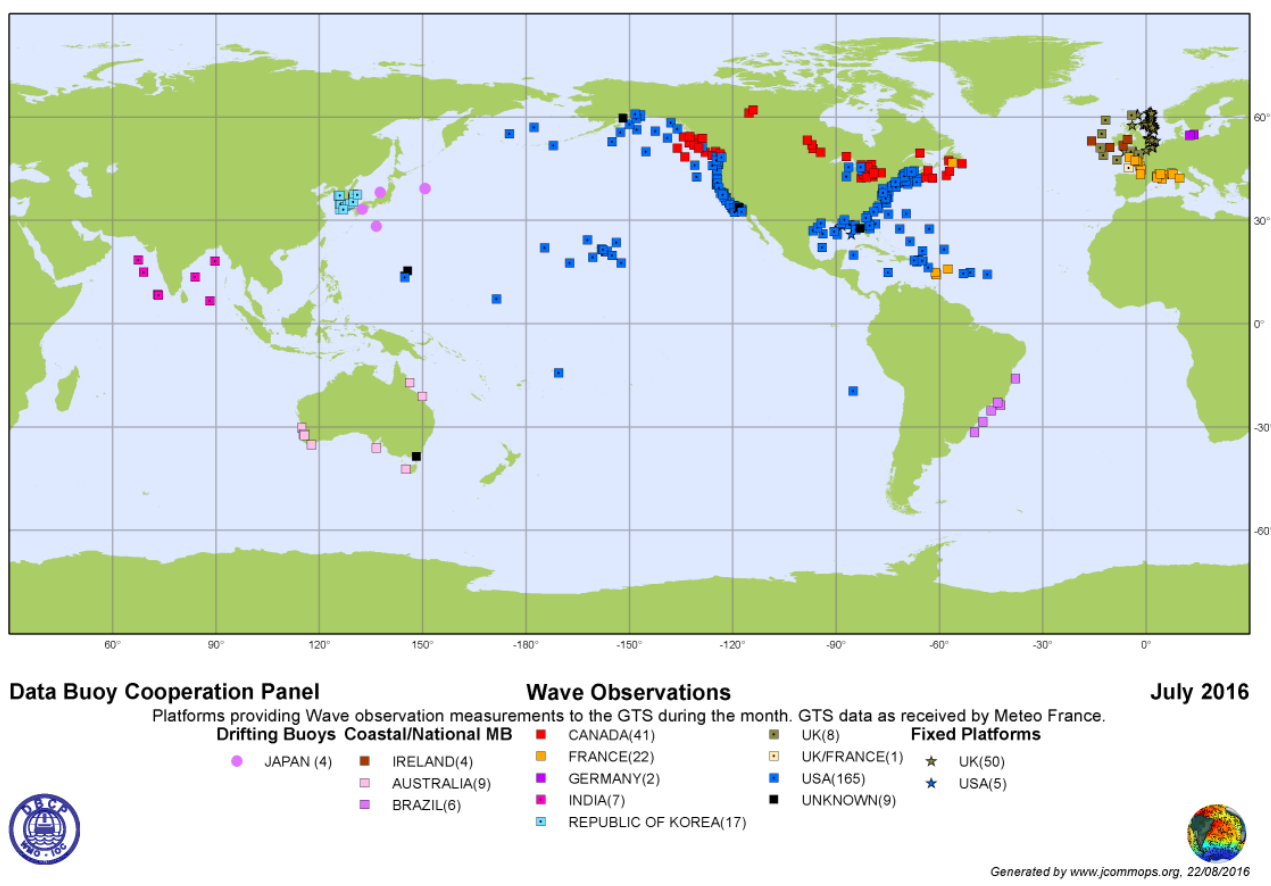

1804 Figure 2.9 - Location of 348, available via GTS, wave measurement sites (July 2016), from JCOMM Data Buoy Collaboration Panel, http://www.jcommops.org/dbcp/network/maps.html .

There are three major components in a buoy wave measurement system: 1) the platform, the hull, shape, composition, super-structure, and mooring; 2) the sensor; 3) the payload or on-board analysis package. Each group has its own unique characteristics that necessitate specific attention, requiring very explicit information and guidance. Surface buoys can be spherical, discus, boat-shaped (e.g. NOMAD, Timpe and Van de Voorde, 1995), with extended super-structures generally housing meteorological sensor packages, solar panels for supplemental power, cages to prevent access by 
1813 sea mammals. The size of discus buoys ranges from slightly more than one meter up to $12 \mathrm{~m}$ in 1814 diameter (but see the two paragraphs at the end of next subsection 2.5.3). The composition of the 1815 hull has been evolving from aluminium to foam. Moorings (and bridling systems) can be open link 1816 chain, polypropylene, and shock cord, permitting the buoy to be free-floating and actively revolving 1817 within a well-defined watch circle. The sensor systems have changed over the past three decades. 1818 HIPPY (see http://www.datawell.nl/Products/Motionsensors.aspx) sensors have been used 1819 operationally in the early 1970's (Steele et al., 1992; Steele et al., 1998). The configuration was a 1820 mechanical gimbaled sensor used to directly determine the pitch and roll angles (or to measure the 1821 accelerations in $\mathrm{x}, \mathrm{y}$, and $\mathrm{z}$ as in the case of a Datawell wave buoy). As in the case of 1822 NOAA/NDBC, the trend over the past two decades has been to migrate from HIPPY sensors to 1823 electronic motion packages housing tri-axial accelerometers combined with digital magnetometers 1824 and compass packages to measure the buoys motion and translate it to the free surface.

Most of the existing (e. g. AXYS, http://axystechnologies.com/ : NOMAD, TRIAXYS, http://www.oceanor.com/systems/seawatch/buoys-and-sensor/ WAVESCAN, and SEAWATCH) and new companies (TIDAS 900 http://www.nortekusa.com/usa/news/new-tidas-900-monitoring-

1830 buoy) now market wave measurement systems capable of providing directional estimates of the free 1831 surface. Testing and evaluations of the buoy technologies, old and new, need to be addressed to 1832 better understand the differences in the wave measurement from one buoy system to the other.

1834 Using their recent advancements, Global Positioning Systems (GPS) have been used as sensors in 1835 wave buoys. The evaluation of the integral wave parameters and frequency spectra was made with 1836 respect to a Datawell Directional Waverider off the California coast (Herbers et al. 2012) and to a 1837 high resolution pressure array located along the outer banks of North Carolina (Thomson, 2012). In 1838 both cases the tests were conducted in a low wave energy environment. These GPS wave buoys use 1839 a specialized sensor package that measures the horizontal and vertical buoy velocities based on the 1840 Doppler shift in received GPS signals providing a more accurate estimate of the free surface.

1842 2.5.3 The truth about wave measurements from buoys

1843 where we analyse the performance of buoys as measuring systems pointing out advantages, 1844 disadvantages and what we can and cannot get from their records 
1846 Surface buoys are the most common and largest number of point source wave measurement systems 1847 occupying enclosed, semi-enclosed water bodies and the coastal waters of the world. Because of 1848 this and the interplay between point-source measurements and altimeter data sets, and their use in 1849 NWP's forecast evaluation, our attention focuses on these systems. This requires a steady effort by 1850 wave modellers to resist the pressing request by forecast management to assimilate the buoy data in 1851 the daily analysis, in so doing improving both analysis and forecast, especially close to the coast, 1852 where most of the buoys are. However, wave modellers want independent non-assimilated wave 1853 data to be able to get an objective judgment of the quality of the model results.

1855 One of the primary objectives is to obtain accurate estimates of the two-dimensional distribution of energy $\mathrm{S}$ in frequency $\mathrm{f}$ and direction $\theta$ shown in Equation (1) below.

$$
S(f, \theta)=S(f)\left[a_{1} \cos \theta+b_{1} \sin \theta+a_{2} \cos 2 \theta+b_{2} \sin 2 \theta+\sum_{n=3}^{\infty} a_{n} \cos n \theta+b_{n} \sin n \theta\right]
$$

This is crucial information, absolutely necessary to be able to evaluate a wave model's performance for any meteorological event(s) at as many measurements sites as possible. Second, a wave buoy containing a sensor measures the buoy response in the presence of free surface waves. Thus, the measured buoy response requires a mathematical transfer function that will allow estimating the free surface. Each buoy configuration should have its own unique transfer function dependent on the physical factors influencing the buoy motion. It is clear this may not be the case in our existing global wave buoy array.

No matter what defines the sensor, the payload (i.e. analysis package) acquires the raw signal and transforms it to an estimate $(\mathrm{x}, \mathrm{y}, \mathrm{z})$ of the free surface from which ultimately directional estimators (the lowest Fourier coefficients $a_{1}(f), b_{1}(f), a_{2}(f), b_{2}(f)$ in the above equation), frequency spectra, and integral wave parameters are derived. For example the Datawell is a particle follower buoy; its estimate of the Fourier coefficients follows directly from the measured accelerations and linear wave theory (O'Reilly et al. 1996), whereas the NOAA/NDBC is a slope following system, and the estimates of $\mathrm{a}_{1}, \mathrm{~b}_{1}$ incorporate various corrections for hull-mooring response (Steele et al., 1992, noted that translational buoys use $\alpha_{1} \alpha_{2}, r_{1}$ and $r_{2}$ that are trigonometrically related to the four Fourier directional coefficients). TRIAXYS systems (MacIsaac and Naeth, 2013) are based on strapped down accelerometers and three strap down rate gyros. 
1880 In general, only the first four moments of the directional distribution can be resolved, the mean 1881 wave direction, the directional spread, the skewness and kurtosis (Jensen et al., 2011). Directional 1882 estimators like the Maximum Likelihood (MLM) and Maximum Entropy Methods (MEM) 1883 approximate the series expansion (the summation in Equation 1) to rectify the directional 1884 distribution (e.g., Benoit, 1992). Inverting the spectral estimates using MEM will reproduce the 1885 four directional Fourier coefficients exactly. MLM will not. Hence, any graphical representation 1886 (Figure 2.10) of the directional shape or 2-dimensional directional wave spectrum is an interpretation of nature, and should not be construed as exact. The only exception would be a multi-component linear array where the number of degrees of freedom permits the direct estimation 1889 of the directional wave spectrum (e.g. Long and Oltman-Shay, 1991).
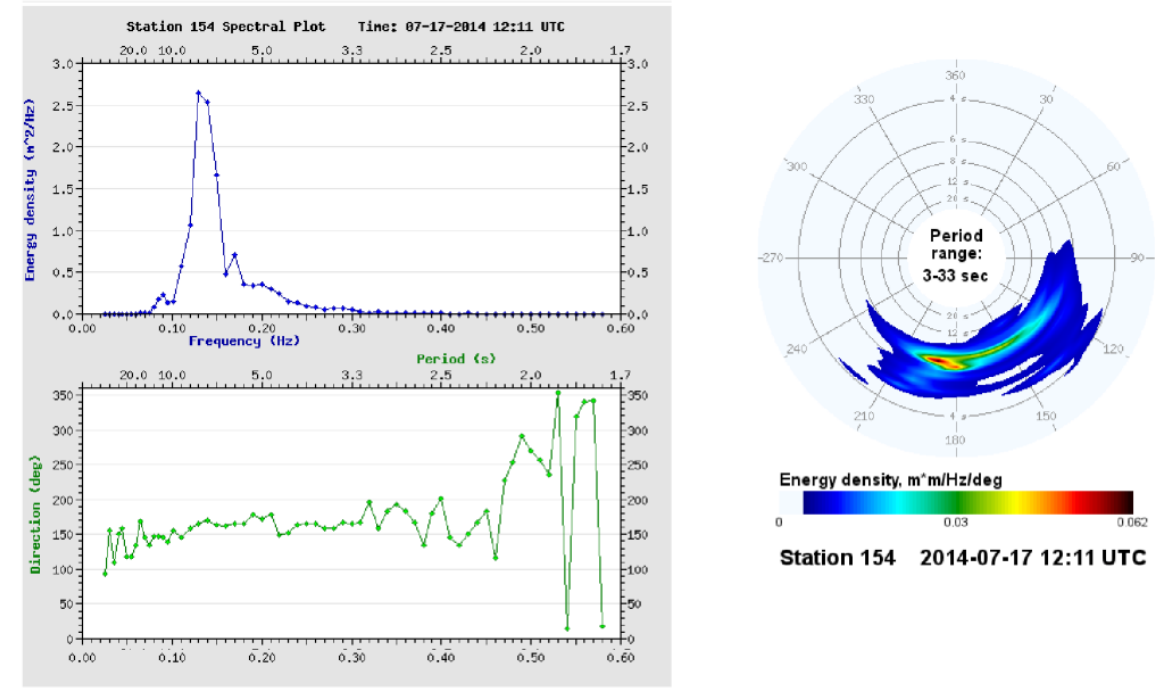

Figure 2.10. (Left panel) frequency spectra and vector mean wave direction, and (right panel) estimate of directional wave spectra, for $\mathrm{H}_{\mathrm{mo}} 1.43 \mathrm{~m}$ from http://cdip.ucsd.edu/?\&nav=recent\&sub=observed (Station 44097).

All wave buoys monitoring the ocean surface contain noise in the time series record, similar to that encountered for pressure gauge, or velocities derived from acoustic profilers in shallow water. It is the level of that noise compared to the real wave energy or in the directional coefficients that can contaminate any wave record. Noise generally resides in the less energetic part, i.e. the low and high frequency range of a spectrum., For the same reason noise is also more apparent in wave records when the natural energy level is small. The signal-to-noise level provides the measure to determine the quality of the wave sensor output. O'Reilly (2007) equated directional wave instruments to audio equipment, defining what we can call fidelity. Instruments with high fidelity 
1903 can be used to resolve some of the finer details of the directional wave spectrum, like the directional 1904 width at a particular frequency and can often determine if the directional characteristics at that 1905 frequency are bi-modal. Low fidelity instruments will generally return reasonable estimates of the 1906 mean wave direction, but will over-estimate the directional spread and under-estimate skewness and 1907 kurtosis (O'Reilly et al., 1996). A common assessment of the signal-to-noise levels is that sea 1908 surface displacement has a larger signal than the sea surface slope signals. Similarly, while an 1909 increased wave energy will result in a larger signal, the lower the frequency the lower the 1910 acceleration, slope, and velocity signals and thus the higher the potential for contamination from 1911 noise. It is a controlled balance between removing noise without removing real energy. Following 1912 a better assessment of the tapering off of low frequency noise in an acceleration spectrum, 1913 NOAA/NDBC has modified their noise correction algorithms (Riley et al., 2011). This can have 1914 non-trivial consequences.

1916 An example of this phenomena (Figure 2.11) is given by buoy data recorded in the eastern end of 1917 Lake Ontario. The interaction between the Canadian Hydraulics Centre of the National Research 1918 Council of Canada and AXYS buoy company led to the development of the TRIAXYS Directional 1919 wave buoy (Kashino, 2011; MacIsaac and Naeth, 2013), characterized by three strapped down 1920 accelerometers and three rate gyros measuring the buoy motion with six degrees of freedom. This 1921 allowed keen measurements also in the low frequency range previously considered void of energy 1922 (because of the dimensions of the lake). The results were enlightening, and they are shown in Figure 1923 2.11. It is obvious that considering the "full spectrum" provides higher wave heights, with 1924 differences up to $0.5 \mathrm{~m}$ that, especially for inner seas, may be a large per cent of the actual signal. 
1926

1927 Figure 2.11. Scatter plot derived from Canadian buoy 45135 located in the eastern end of Lake 1928 Ontario. The comparison is between full spectrum analysis and assuming no energy below $0.12 \mathrm{~Hz}$. 1929

1930 Noise in wave records in the coastal area may also be manifested in larger scale physical processes 1931 such as near-inertial surface currents. Gemmrich and Garrett (2012) investigated long-term wave 1932 height records (hourly estimates over a 20-yr period of record) along the Canadian coast, ranging 1933 from near coastal observations to about $700 \mathrm{~km}$ from shore. They found a distinct peak in the $\mathrm{H}_{\mathrm{mo}}$ 1934 power spectra by periodic currents. Given a clearly defined peak, the contribution to the overall 1935 variance in $\mathrm{H}_{\mathrm{mo}}$ was small, on the order of 0.03-percent in the variance at the inertial peak compared 1936 to the background variance of the wave climate. The average root-mean-square modulation of the 1937 wave height by near-inertial currents was 2-percent.

1938

1939 The most dramatic study of buoy records was performed by Bender et al. $(2009,2010)$. A 3-m 1940 discus buoy (similar to the standard NOAA/NDBC 3-m buoy), containing multiple sensor 1941 packages, was deployed during Hurricane Katrina in the Gulf of Mexico. This study found large 1942 differences (26 to 56\%!) in significant wave height estimates as the wave heights increased from 6 1943 to $8 \mathrm{~m}$, the differences depending on the analysis methods used. Strapped down accelerometers 1944 commonly used by the world's wave measurement providers would be affected by this work. It 1945 would also have a dramatic impact on measuring extreme wave conditions not only from tropical 1946 cyclone forcing, but also large extra-tropical events.

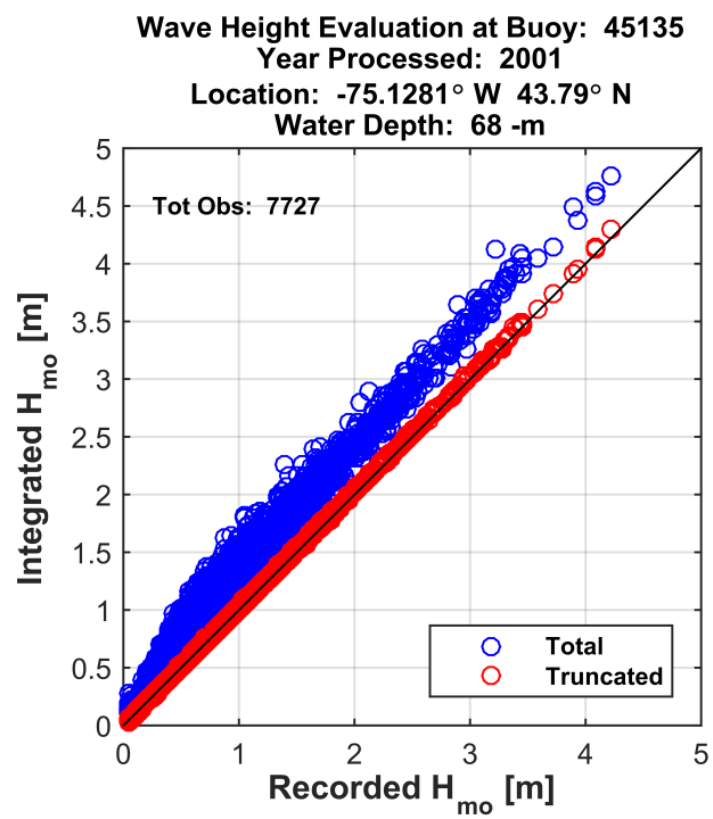


1948 The sustained heeling of the buoy due to wind forcing on the super-structure was cited as the likely 1949 cause of the bias. NOAA/NDBC is now implementing a suggested corrections of Bender et al. 1950 (2010) on their 3-m buoy systems to remove this effect in extreme situations that on the other hand 1951 are the most interesting ones to have data about (Jensen et al., 2011). Collins et al., (2014) applied 1952 the tilt correction suggested by Bender et al., (2010) during the Impact of Typhoons on the Ocean in 1953 the Pacific (ITOP) experiment for wave data measured from a $6 \mathrm{~N}$ buoy. The tilt correction 1954 surprisingly resulted in decreasing and increasing the calculated surface elevation signal from wave 1955 to wave. However, in the extreme case the tilt correction did in fact reduce the individual wave 1956 height estimates. The 6N buoy used in ITOP (Drennan et al., 2014) was deployed in a water depth 1957 of $5600 \mathrm{~m}$, with an approximate watch circle of $1.45 \mathrm{~km}$. Wind and wave loading derived from the 1958 passing of four typhoons with wind speeds of $25 \mathrm{~m} / \mathrm{s}$ and $\mathrm{H}_{\mathrm{mo}}$ values of $10 \mathrm{~m}$ may not push the buoy 1959 to its mooring limit reducing the tilt. Results from Bender et al., (2010) 3D buoy were based on a 1960 deployment in a depth of 19m and most likely a very small watch circle. It seems likely Bender's 1961 buoy would be more susceptible to adverse tilt because of the mooring length compared to that of 1962 IOP. From the two cases it is apparent that the problem of tilt is mostly felt in coastal water, often 1963 shallow enough for the mooring force components to act on a more horizontal direction and, if 1964 shallow enough, waves to be more frequently breaking.

1966 We have purposely focused our attention on the "traditional" buoys, i.e. the ones with diameter $\geq$ $19671 \mathrm{~m}$. However, the progresses in electronics, data storage and energy supply have led in the latest 1968 years to a drastic reduction of the related volume and weight. The obvious consequence has been a 1969 reduction of also the hull (dimensions and weight) and cost, making these buoys easily handable 1970 from a small boat.

1972 A number of these buoys is now on the market, as the SCRIPPS buoy 1973 (http://iprc.soest.hawaii.edu/NASA_WS_MD2016/pdf/Centurioni2016.pdf), SWIFT by the 1974 University of Washington (http://www.apl.washington.edu/project/project.php?id=swift), and 1975 Spotter by Spoondrift (https://spoondrift.co). The problem is that these buoys have not been 1976 extensively tested in all the possible conditions. A test concerning the electronics would be 1977 straightforward, putting them on the same hull as done (see above) by Bender et al. (2009, 2010). 1978 However, the main concern with these light buoys is their hydrodynamic response. For instance, it 1979 is natural to expect that a steep, or even breaking, wave crest will tend to move horizontally the 1980 buoy at a non-trivial extent, the more so when the buoy is drifting. The point is that the limited cost 1981 makes the buoys expendable, letting them drift (while transmitting data) till when they last or run 
1982 aground. Tests can be organised mooring them to one of the "traditional" buoys or another wave 1983 recording system. However, the limited weight suggests that their behaviour when moored or 1984 drifting can be quite different.

\subsubsection{Importance of wave measurement evaluations}

1987 where we stress the importance of assessing the data accuracy and describe the efforts done in this 1988 respect

The increasing quality of wave modelling results and the need for more accurate forecasts force buoys, and the other measuring systems, to a continuous improvement of their performance. In particular there is an acute need for an improved convergence of the data from the various data providers. In 2007 a meeting was held (ACT, 2007) to discuss sensor technologies and evaluations.

1994 This meeting consisted of numerous private sector manufacturers, data providers and data users. 1995 The primary objectives of this workshop were to define the present state of wave measurement technologies, to identify the major impediments to their advancement, and to make strategic recommendations for future developments, and the necessary steps to integrate wave measurements sensors into operation coastal ocean observing systems. Two recurring themes of the discussions were a disparity between user requirements, and to what degree existing and new technologies should be adequately tested. The concept of "First 5" was introduced (O'Reilly, 2007) to nominally evaluate directional estimates in wave measurement systems. As discussed earlier (Equation 1), the "First 5" define the first four directional Fourier coefficients in the infinite series expansion quantifying the directional resolution. The ideas initiated at this meeting were elevated to an international forum through the World Meteorological Organization (WMO) and the Joint 2005 Technical Commission for Oceanography and Marine Meteorology (JCOMM) at a meeting held in 2008 (JCOMM, 2008). Ultimately this carried forward resulting in the Integrated Ocean Observing System Report entitled A National Operational Wave Observation Plan (IOOS, 2009, and the related summary by Birkemeier et al., 2012). Swail et al. (2009) summarized the need for high quality directional wave measurements and further acknowledged the protocols for intrameasurement evaluations. These procedures were summarized in Jensen et al. (2011), where a 2011 relative reference (a Datawell Directional Waverider buoy) was selected to serve as the baseline for 2012 the evaluations, and a methodology was introduced (WaveEval Tools, see 2013 http://cdip.ucsd.edu/?nav=documents\&sub=index\&xitem=product\&xdoc=cdiptool ) to serve as the 2014 method for the evaluations. ACT (2012) followed their original workshop establishing a plan, later 
2015 summarized by Luther et al. (2013), for the execution of the intra-measurement investigations, and 2016 the procedures to follow.

2018 The concept of an intra-measurement evaluation is not new. Over the past twenty-five years there 2019 have been a series of experiments at various locations focusing on the evaluation of wave 2020 measurement systems (e.g. WADIC, Allender et al., 1989; Harvest Platform, O'Reilly et al., 1996; 2021 Wacsis, van Unen et al., 1998; FETCH, Pettersson et al., 2003; NDBC Sensor Systems, Teng and 2022 Bouchard, 2005; ITOP, Collins et al., 2014) and analysis methods to use in the evaluation process, 2023 Krogstad et al. (1999). As new sensor and wave measurement systems mature, the need for more 2024 intra-measurement evaluations will emerge.

One study, the Field Laboratory for Ocean Sea State Investigation and Experimentation (FLOSSIE) located in Monterey Canyon off the California coast, was initiated in July 2015 (Jensen, et al., 2015). Two primary motivating factors for this experiment were to investigate findings from Durrant et al. (2009) suggesting the wave buoy networks of NOAA/NDBC and Environment Canada differed by as much as 10-percent when compared to altimeter estimates. The second was based on a need to determine the quality in NOAA/NDBC wave estimates derived from $6 \mathrm{~N}$ buoy systems from their original deployments in the mid 1970's to the present. The need was exacerbated by NOAA/NDBC plans to retire these large buoy platforms. Based on historical records, (NOAA/NDBC and Environment Canada) there are 100's of years of wave records reported from $6 \mathrm{~N}$ systems, and the quality of the data is ill defined.

Table 2.3 Wave Estimates from Maximum and Wind Wave Growth During FLOSSIE

\begin{tabular}{|c|c|c|c|c|c|c|}
\hline \multirow[b]{2}{*}{ Storm } & \multirow[b]{2}{*}{ Buoy ID } & \multicolumn{2}{|c|}{ Winds (Averaged) } & \multicolumn{3}{|c|}{ Wave Measurements } \\
\hline & & $\begin{array}{l}\text { Wind Speed } \\
(\mathrm{m} / \mathrm{s})\end{array}$ & $\begin{array}{l}\text { Wind } \\
\text { Direction }\end{array}$ & $\mathrm{H}_{\mathrm{mo}}$ & $T_{p p}$ & $\boldsymbol{\theta}_{\text {wave }}$ \\
\hline \multirow[t]{7}{*}{ Maximum } & $6 \mathrm{~N}$ Inclinometer & 6.4 & 259 & 9.01 & 16.67 & $\mathrm{~N} / \mathrm{A}$ \\
\hline & 6N 3DMG & & & 8.86 & 17.39 & 308 \\
\hline & 6N HIPPY & & & 8.67 & 17.39 & 316 \\
\hline & 6N-AXYS-Triaxys & & & 9.08 & 16.67 & 291 \\
\hline & 3D-3DMG & 5.9 & 244 & 8.75 & 17.39 & 285 \\
\hline & 3D-HIPPY & & & 8.92 & 17.39 & 288 \\
\hline & DatawellWaverider & & & 7.96 & 18.18 & 300 \\
\hline \multirow[t]{3}{*}{ Growth } & $6 \mathrm{~N}$ Inclinometer & 4 to 12 peak & 157 (18hr) & 2.39 & 20.00 & $\mathrm{~N} / \mathrm{A}$ \\
\hline & 6N 3DMG & & & 2.32 & 19.05 & 170 \\
\hline & 6N HIPPY & & & 2.20 & 19.05 & 164 \\
\hline
\end{tabular}




\begin{tabular}{|l|l|l|l|l|l|l|}
\hline & 6N-AXYS-Triaxys & & & 2.34 & 18.18 & 186 \\
\hline & 3D-3DMG & 4 to 12 peak & 146 (09hr) & 2.37 & 19.05 & $\mathbf{1 9 0}$ \\
\hline & 3D-HIPPY & & & 2.35 & 18.44 & $\mathbf{2 0 9}$ \\
\hline & DatawellWaverider & & & 2.30 & 18.18 & $\mathbf{2 3 7}$ \\
\hline
\end{tabular}

2040 One of the most important outcomes of this study is that, despite consistency in the wave height, 2041 period and direction (only relative to the 3D buoy) measurements (Jensen et al., 2015), there is a 2042 substantial variation in the frequency spectra and frequency dependent directional components 2043 (mean wave direction, spread, skewness and kurtosis). Two examples illustrate this point: the 2044 maximum wave height found in the one year wave record and local wind-wave growth with an 2045 accompanying swell system at peak wind speed. The results are summarized in Table 2.3.

2047 The integral parameters from the maximum event data set show a relative consistency in significant wave height, $\left(\mathrm{H}_{\mathrm{mo}}\right.$, maximum difference of $1.1 \mathrm{~m}$ between the AXYS-Triaxys and Datawell Waverider), parabolic fit wave period $\left(\mathrm{T}_{\mathrm{pp}}\right.$, weighted average of the energy on either side of the peak frequency) difference of about 1.3-s (Datawell versus 6N inclinometer and AXYS Triaxys), and the direction of about $30-\operatorname{deg}\left(\theta_{\text {wave }}\right)$. In general, the nearly one year of wave records show a similar trend. The directional estimates derived from the 6N-HIPPY, 6N-3DMG and 6N-AXYS are estimated and tabulated despite the consensus suggesting directional wave characteristics cannot be obtained from non-symmetric buoy hulls. The only reason for relatively good estimates is that the predominant direction for winter storms along the Pacific coast span about \pm 45 -deg off of central angle of 315-deg. The local wind conditions are relatively low, and about 90-deg off the mean wave direction angle, potentially offering up a secondary southwesterly wind-generated wave system nearly hitting the $6 \mathrm{~N}$ broadside.

The frequency spectra from the maximum event based on the integral wave parameters and winds during the time should be relatively smooth, single peaked, or possibly a secondary peak in the high frequency range. Each of the seven spectra derived from the seven sensors are found in Figure 2.12, panel a. Each spectrum is defined by its discrete frequencies, interval and range. For convenience and illustration purposes the upper limit in frequency is truncated at $0.3 \mathrm{~Hz}$ to better show the differences or similarities in the lower range. The energy spectra for all cases above the $0.3 \mathrm{~Hz}$ limit were close to zero, and displayed no signs of a secondary wave system. Spectra from the $6 \mathrm{~N}$ sensors are plotted in red. With the exception of the $6 \mathrm{~N}$-Inclinometer the spectral estimates are similar. The deviation in the inclinometer spectra is most likely a result of a $\Delta \mathrm{f}=0.01-\mathrm{Hz}$ 

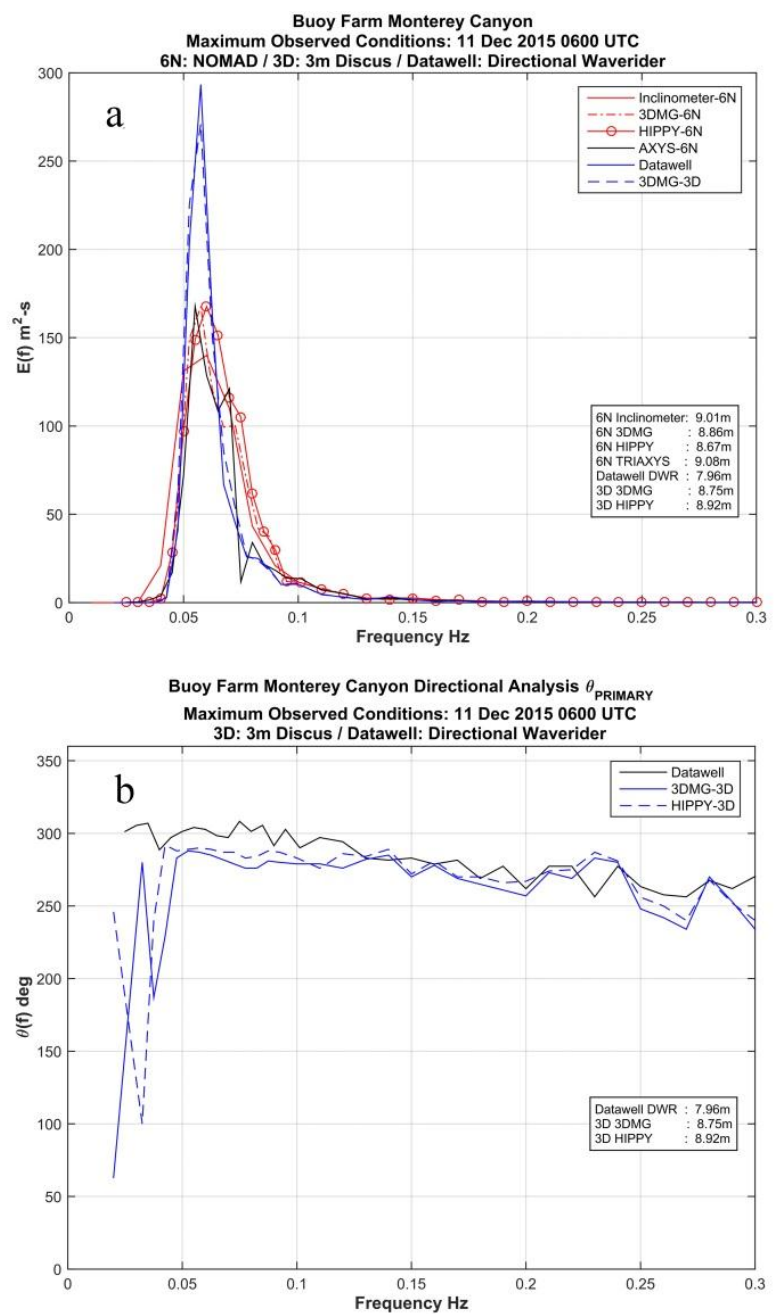

2071 Figure 2.12. a) Frequency spectra, b) vector mean wave direction, from seven sensor/payload 2072 systems occurring at the maximum event during FLOSSIE.

2074 while the other sensor systems are set at $0.005-\mathrm{Hz}$ for frequencies less than about $0.09-\mathrm{Hz}$. The 2075 forward, peak and rear face of the 6N-3DMG, HIPPY, and AXYS-Triaxys display only slight 2076 variations from one another, while the 6N-Inclinometer contains more energy in the forward and 2077 rear face of its spectra. For the other two independent buoy systems (NOAA 3D, and Datawell 2078 Waverider), the spectra differ. The Waverider (black line) $\mathrm{E}\left(\mathrm{f}_{\mathrm{m}}\right)$ is shifted slightly toward a lower 2079 frequency, but the magnitude is similar to the $6 \mathrm{~N}$ data. The rear face of the Waverider spectra 2080 relative to the $6 \mathrm{~N}$ data sets shows why the Waverider $\mathrm{H}_{\mathrm{mo}}$ is lowest compared to all results. The 2081 second independent site located about $11 \mathrm{~km}$ northeast of FLOSSIE, and $8 \mathrm{~km}$ northwest of the 2082 Datawell show the greatest deviation in the spectra (solid and dashed blue lines) relative to 2083 FLOSSIE and the Datawell, however both are very similar. The peak energy is nearly a factor of 2084 two greater, more peaked about $\mathrm{E}\left(\mathrm{f}_{\mathrm{m}}\right)$, and the rear face energy level falls more dramatically. In 
2085 arithmetic space the rear face slopes from the 3D site differ, however in log-log space they 2086 converge on the $6 \mathrm{~N}$ results. The two sensors and payloads (analysis packages), contained in the 3D 2087 hull are identical the sensor/payload packages in the $6 \mathrm{~N}$. The moorings are also similar. It seems 2088 obvious hull differences contribute to the spectral characteristics found in Figure 2.12.

2090 Directional analysis for the maximum condition example is restricted to the data from the 3D and 2091 Datawell buoys. Despite having the capability to obtain the four Fourier directional components 2092 from the HIPPY, 3DMG and AXYS-Triaxys sensors on-board the 6N buoy, the results could be 2093 meaningless because of the limitations in capturing directions from the non-symmetrical 2094 characteristics of the buoy itself. Figure 2.12 (panel b) depicts the mean wave direction for each 2095 frequency for the Datawell (black line), the 3D-HIPPY (blue dash line) and 3D-3DMG (solid blue 2096 line). The frequency range is extended to define all discrete frequency bands of the spectra. The 2097 vector mean wave directions (defined at the spectral peak frequency) from the three data sets are 2098 listed in Table 2.3. There are similarities and differences between buoy data sets over the frequency 2099 range. In the low frequency range the 3D sensors are subject to signal to noise, and unable to 2100 rectify a true wave system like that found in the Datawell. Between 0.04 to $0.1-\mathrm{Hz}$, where the bulk 2101 of energy resides in the frequency spectra (Figure 2.12, panel a), the 3D sensors differ about 25-deg 2102 relative to the Waverider. Moving to higher frequencies, the wave direction is aligning with the 2103 local wind direction at about 250deg. In the high frequency range, there appears to be limited noise 2104 in the data despite the relatively low magnitudes in the energy. This example illustrates how, 2105 despite a single peak spectra derived from a storm most likely in the North Pacific, dominated by 2106 swell energy, there is a 50-deg variation in the mean wave direction over the range of frequencies. 2107 The single value defined by the overall mean wave direction will not adequately define the true 2108 wave conditions at these sites.

2110 The second example is somewhat more complex, where there are two wave systems converging on 2111 FLOSSIE. The wave climate is defined by low frequency swell energy derived from a distant storm 2112 in the North Pacific while a locally generated wind-sea is developing. The local wind event 2113 consists of an increasing wind speed while the direction remained nearly constant for 18 hours. 2114 The combined conditions produced wave heights around 2.2 to $2.4 \mathrm{~m}$ observed for the seven sensors 2115 (Table 2.4). The $\mathrm{T}_{\mathrm{p}}$ estimates are from 18 to 20s reflecting the swell energy (Table 2.3). The vector 2116 mean wave direction estimates from the 3D and Datawell show differences as large as 45-deg. 2117 Analyses can be performed using the mean wave period and peak spectral period, where if the 2118 deviation between the two parameters is large, it would suggest multiple wave systems. 
2119 Conversely, if the differences are relatively small, the likelihood of a dominant single wave system 2120 would exist. For the local wind-wave growth examples two wave systems exist, and their 2121 respective significant wave height estimates are displayed in Table 2.4. The four sensors in the $6 \mathrm{~N}$ 2122 buoy suggest the swell energy dominates, while at the 3D and Datawell the wind-sea overshadows 2123 the swell. The differences are not extreme, but are in fact relevant.

2125 The spectral estimates from the seven sensor systems for the local wind-wave growth example are 2126 presented in Figure 2.13, (panel a). Two wave systems exist, a swell component, and a wind-sea 2127 component. All seven sensor systems generally define the wind-sea and swell fairly well, but 2128 there are noticeable differences in the shape and structure. The four sensors on-board the $6 \mathrm{~N}$ buoy 2129 are generally consistent. The Inclinometer and 3DMG spectra are nearly identical, nearly matching 2130 the energy maximum (and frequency band) for both the swell and wind-sea. The AXYS-Triaxys 2131 and HIPPY-6N are similar. The 3D HIPPY and 3DMG spectra follow an analogous trend, and 2132 again are more peaked for the swell system as in the previous example. The Datawell spectra 2133 closely follow the two sensor systems contained in the 3D buoy. The wind-sea portion is however 2134 displaced toward higher frequencies, yet the $\mathrm{H}_{\mathrm{mo}}$ is nearly identical to both 3D results (Table 2.4). 2135 Results for the first directional moment (vector mean wave direction as a function of frequency) are 2136 presented in the panel $b$ of Figure 2.13. As in the previous case only the Datawell and 3D HIPPY 2137 and 3DMG sensor results are plotted. Despite matching the energy of the swell for the three 2138 systems, there are substantial differences in the directional estimates. Below 0.04-Hz, where the 2139 energy level is low, hence carrying a significant amount of noise, the directional estimates are 2140 contaminated, and represented by the large oscillations. Between 0.05 to about $0.065-\mathrm{Hz}$ the 2141 frequency spectra for the three systems compare favourably, however the directional estimates 2142 derived from the HIPPY sensor diverge 

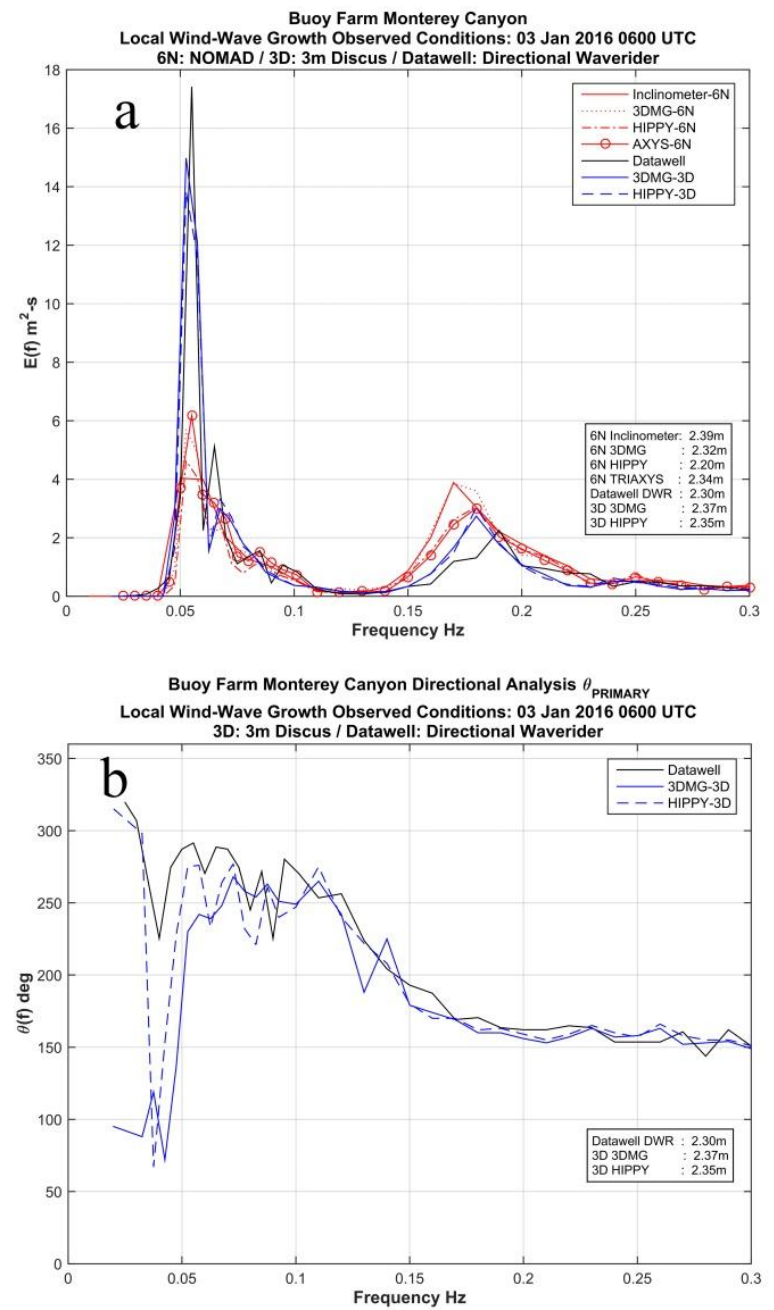

2145 Figure 2.13. a) Frequency spectra, b) vector mean wave direction, from seven sensor/payload 2146 systems occurring at the peak of a local wind-sea event during FLOSSIE.

\begin{tabular}{|l|l|l|l|}
\hline \multirow{2}{*}{ Buoy Id } & \multicolumn{3}{|l|}{$\mathbf{H}_{\text {mo }}(\mathbf{m})$} \\
\cline { 2 - 4 } & Wind-Sea & Swell & Total \\
\hline 6N Inclinometer & 1.89 & 1.46 & 2.39 \\
\hline 6N 3DMG & 1.86 & 1.39 & 2.32 \\
\hline 6N HIPPY & 1.80 & 1.27 & 2.20 \\
\hline 6N-AXYS-Triaxys & 1.82 & 1.48 & 2.34 \\
\hline 3D-3DMG & 1.48 & 1.77 & 2.37 \\
\hline 3D-HIPPY & 1.46 & 1.86 & 2.35 \\
\hline DatawellWaverider & 1.50 & 1.82 & 2.30 \\
\hline
\end{tabular}

2151 from both the Datawell and 3DMG by as much as 50-deg. The directional oscillations from 0.065 2152 to $0.075-\mathrm{Hz}$ match the oscillations in the frequency spectra (Figure 2.13, panel a). These 
differences could be attributed to the separation distances between the buoys, or slightly different sampling times. All three sensors show a similar directional shift in the transition from swell to wind-seas. In the wind-sea frequency range $(0.125-0.4-\mathrm{Hz})$, where the peak frequency is $0.19 \mathrm{~Hz}$

2156 for the Datawell and $0.18 \mathrm{~Hz}$ for the 3D HIPPY and 3D 3DMG, the mean wave direction differences are on the order of $10-\mathrm{deg}$, and much lower than the maximum wave condition example. The directional estimates above $0.4-\mathrm{Hz}$ again diverge because of low energy levels $\left(0.08 \mathrm{~m}^{2} \mathrm{~s}\right)$ similar to that encountered in the low frequency range.

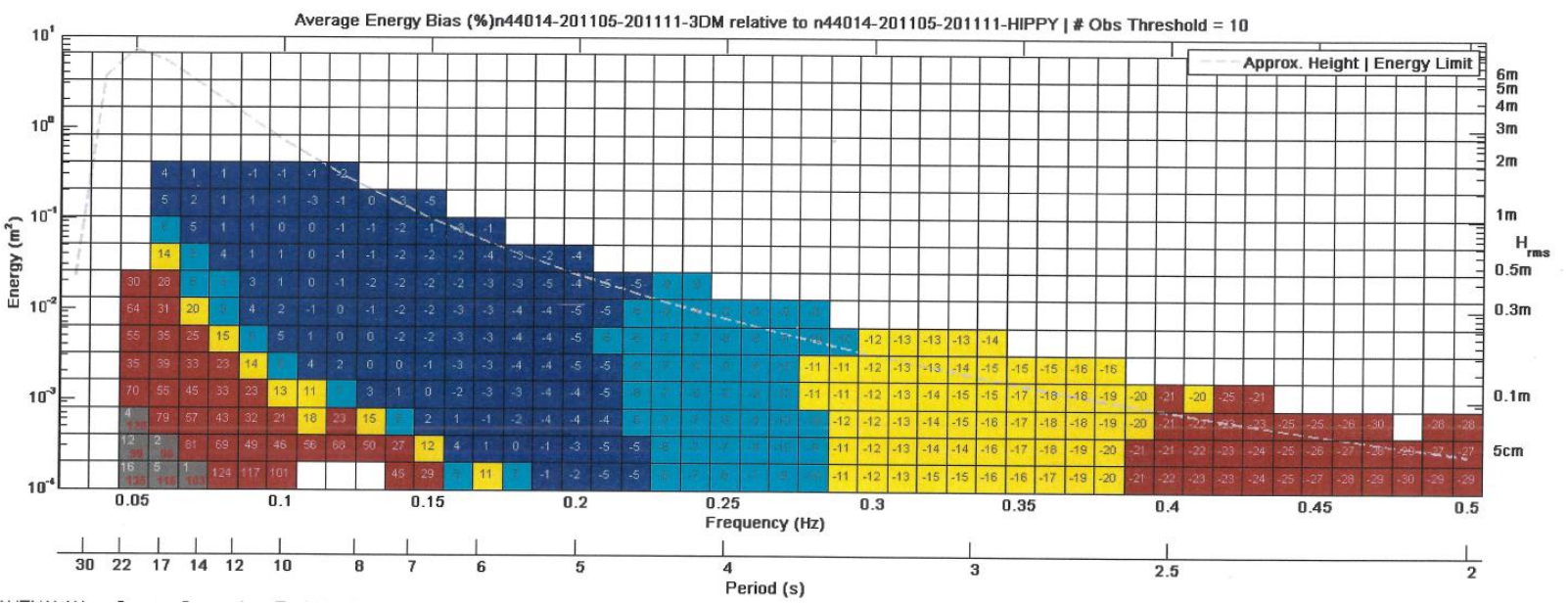

2162 Figure 2.14. Different performance of the two measuring systems, 3DM and HIPPY. The figure provides their average per cent difference (with respect to the latter) as a function of frequency $\mathrm{f}$ and energy $\mathrm{E}$ (this as also $\mathrm{H}_{\mathrm{rms}}$ on the right scale). The specific figure is written in each (f-E) pixel, the pixels then grouped with different colours in the sequential ranges provided in Table 2.4.

More than the above examples, the different performance of the two measuring systems, 3DM and HIPPY, is represented in Figure 2.14 where we show their average per cent difference (with respect to the latter) as a function of frequency $\mathrm{f}$ and energy $\mathrm{E}$ (this as also $\mathrm{H}_{\mathrm{rms}}$ on the right scale). The specific figure is written in each (f-E) pixel, the pixels then grouped with different colors in the sequential ranges provided in Table 2.5. The results are clear. The two systems, 3DM and HIPPY, provide on average the same energy (within 10\% difference) for wave periods longer than $3 \mathrm{~s}$, but

2173 from $6 \mathrm{~s}$ upwards only if there is enough energy in the system. HIPPY is much lower in energy for 2174 long period but not large waves. Conversely its energy is always larger in the upper frequency 2175 range.

Table 2.5 - Colour to range identification

\begin{tabular}{|l|l|}
\hline color & range (percent) \\
\hline dark blue & $0 \%$ to $\pm 5 \%$ \\
\hline
\end{tabular}




\begin{tabular}{|l|l|}
\hline light blue & $\pm 5 \%$ to $\pm 10 \%$ \\
\hline yellow & $\pm 10 \%$ to $\pm 20 \%$ \\
\hline red & $> \pm 20 \%$ \\
\hline grey & number of observations below threshold \\
\hline white & no data \\
\hline
\end{tabular}

2178 Despite FLOSSIE residing in deep water, the information gained from an experiment like this can

2179 be applied to the coastal domain, including continental shelves and near-shore regions, as well as 2180 semi-enclosed and enclosed water bodies. In general, for coastal applications the water depth will 2181 influence any wave measurement. A buoy is deployed at a unique site defined by a latitude 2182 longitude pair, however there is a defined watch circle (based on the mooring configuration), the 2183 buoy will meander while it measures the local meteorological, oceanographic and waves conditions. 2184 In the coastal domain, the importance of where the buoy is stationed relative to the local bathymetry 2185 becomes extremely important. Finite depth mechanisms such as shoaling and refraction will affect 2186 the measurements and are frequency dependent affecting the energy and directional attributes. 2187 Provided that the transfer function relating the buoy motion to the free surface is well composed, 2188 the quality in the data will be retained. Water level variations based on tides and surge effect as 2189 well as currents will impact the wave measurements more so in the coastal region, and could 2190 contaminate the data. Performing a FLOSSIE type experiment in finite depths would most likely 2191 yield larger differences in the five Fourier directional variables. In semi- and enclosed bodies of 2192 water the only external factor affecting the wave measurements is the marine/land boundary. The 2193 wave climate will be dictated by the size of the domain; the smaller this is the more the applicable 2194 frequency range will be translated toward higher frequencies. Sampling rates will have to be 2195 shorter to compensate for the high frequency waves. Noise levels for the high frequency range of 2196 observable conditions needs to be reduced. And finally measuring high frequency range common 2197 to small water bodies requires the buoy size be small enough to adequately resolve those conditions.

2199 One other aspect found in the examples shown illustrates the need to better evaluate intra-wave 2200 measurements as well as model to measurements. Defining the wave conditions using only the 2201 significant wave height, period and mean direction only answers the basic tendencies at a site. The 2202 details found in the frequency spectra and, if provided, the directional attributes defined by the four 2203 Fourier directional parameters result in a better picture of the existing wave conditions. Two 2204 examples illustrate the similarities and differences between sensor systems, hull types and analysis 2205 packages that lay behind a set of standard values of $\mathrm{H}_{\mathrm{mo}} \mathrm{T}_{\mathrm{pp}}$, and $\theta_{\text {wave. }}$ For multiple wave systems 2206 one system will be ignored. The energy will be combined into the larger of the two. Partitioning 
into a wind-sea and swell wave system is a better method than using only one (see in this respect

2208 Chapter 4). Unfortunately the wave community continues to be steadfast in the use of a three 2209 variable system. Whether it is reporting the quality in our ability to accurately forecast waves, or a 2210 wave model performance evaluated for a single or series of storm events, or intra-measurement 2211 evaluations time, scatter graphics along with statistical tests based on the height, period and wave 2212 direction are usually presented. Even standard statistical tests as in the case of the root mean square 2213 error (and normalized), scatter index used in evaluation of waves has recently been questioned, 2214 (Mentaschi et al., 2013).

2216 One year of hourly wave directional wave measurements define a population over 8,500 individual 2217 observations. Considering the number of frequencies in our present measurement capabilities of 2218 about 50, where energy density and four directional moments (or four Fourier directional 2219 parameters) are defined, the amount of data to investigate becomes large, but not insurmountable. 2220 However, that information defines better what exists at a given site. Over the years attempts have 2221 been made to reduce the number of independent variables, and yet provide results to quantify the 2222 details in the directional wave spectra. Spectral partitioning was introduced to the wave community by Gerling (1992) with follow-on studies by Hanson and Phillips (2001), and more recently by 2224 Portilla et al. (2009) and Portilla et al. (2015). The requirement for these methods is to define 2225 directional wave spectra. As previously noted, other than possibly a high resolution (up to 10 sensors) linear array, the best a buoy or current profiler can provide is the energy density and four Fourier directional parameters as a function of frequency. Hence, spectral partitioning is dependent on an estimate of the directional distribution that approximates the infinite series found in Equation 1. Gerling (1992) and Hanson and Phillips (2001) assumed a directional distribution, whereas Portilla et al. (2015) interrogated the frequency spectra defining energy peaks and a filter consisting of a two-dimensional discrete convolution operation between the spectrum and an equally weighted convolution kernel. WaveEval Tools (Jensen et al., 2011 and ACT, 2007) take a different approach. The four Fourier directional parameters are used to calculate the four moments of the directional distribution at each frequency band: the mean direction, spread, skewness and kurtosis. Partitioning is performed on each discrete frequency band, and a discrete energy level. A bias and root mean square error percentage is determined from averaging the differences between two data sets. The result is a qualitative graphic displaying defined range of the per cent deviations. These techniques can provide useful information that is quantitative as well as qualitative reducing the assessment in directional properties to a reasonable number of products. Recently, two new methods evaluating frequency spectra (Dabbi et al., 2015), and correlating paired wave spectra 
2241 (Collins et al., 2015) have been used. The work by Dabbi et al. (2015) introduced a seven pair 2242 estimator to better define unimodal wave spectra. Although somewhat limited to unimodal spectra, 2243 it does provide an alternative to using the bulk wave height, period and direction estimates. On the 2244 other hand, Collins et al. (2015) used a canonical correlation analysis to investigate the correlation 2245 structure of the matrix of spectral correlations. They found the method was effective to understand 2246 the degree of correlation between sets of paired spectral observations.

2248 There is no lack of trying to develop new methods to evaluate large spectral data sets to determine 2249 similarities, differences, quality or deficiencies in measurement to measurement systems, model to 2250 model results or model to measurements. However, we cling tightly to the bulk wave parameters 2251 because we know what they represent. For example two data sets produce a bias of $0.5 \mathrm{~m}$ out of $4 \mathrm{~m}$. 2252 We know what that represents; we know how large a $0.5 \mathrm{~m} \mathrm{H}_{\mathrm{mo}}$ looks like. Now consider a 2253 difference in the frequency spectra of $10 \mathrm{~m}^{2} \mathrm{~s}$ out of $125 \mathrm{~m}^{2} \mathrm{~s}$. The ratio is the same as in the case of 2254 the $\mathrm{H}_{\mathrm{mo}}$, but what does it represent? That may be the only impediment holding the wave 2255 community back from progressing into the future. An intermediate solution is the use of partitions 2256 (see more in Chapter 4) where we split a full spectrum into the single composing, and at a large 2257 extent independent, wave systems. Then the use of integral parameters makes more physical sense, 2258 and it is much more intuitive to mentally combine different and well defined wave systems coming 2259 together at the considered point.

2261 Establishing a "First 5" criterion and a well sought analysis procedure to evaluate present and future 2262 directional wave measurements is a lofty goal, and will impact nearly every facet in the study of 2263 wind generated surface gravity waves from a physics based standpoint, to model improvements and 2264 daily performance of our weather prediction forecast centres. To have some quantifiable standard 2265 for all wave measurements would be highly beneficial to the user, and thus remove existing 2266 uncertainties, generally dismissed to the level where all data are at a uniform quality level, 2267 something far from the truth. 


\section{6 - Applications}

where we provide examples of wave modeling application in coastal and enclosed seas

2279 environments, showing a sample of the different problems/situations we have to face compared to 2280 the open ocean.

2282 In the previous sections we have listed and discussed the characteristics that, for a certain range of 2283 problems, make wave modeling in coastal and enclosed seas different from the open oceans. These 2284 concern, possibly most of all, the meteorological input due to a potentially strong influence of land 2285 and its orography. Being the inner seas more dominated by wind sea than in the ocean, the wind is 2286 often the crucial aspect of an application. The other relevant aspect is the presence of a variegated coastline and the limited depths waves have to deal with. This leads to a number of complications concerning both physics (for the correct representation of the processes involved) and numerics. The latter derives for large part from the frequent strong spatial gradients of the fields that imply particular limitations, hence attention, in the methods we use to integrate the model equations to obtain what we would like to be reliable and accurate results. All these problems have been analyzed and discussed in the first four sections of this chapter. Then of course we wish to know how correct our results are, and this is achieved comparing them with the measured truth. Unluckily 2294 (Section 2.5) measured data turn out not to be (within limits) the solid reference we would like to 2295 have. Different instruments, also of the same kind, have different problems and accuracy, and this 2296 has to be considered in the validation of model results.

2297 Having framed the spirit of the problem, in this section we provide examples dealing with different 2298 aspects:

22992.6 .1 - the NOAA/NCEP multiple system for the whole US coastline,

23002.6 .2 - the Adriatic Sea and the enhancement of wind speed,

23012.6 .3 - wave forecast for the Rotterdam channel,

2302 2.6.4 - the fractal coastline of the Botthnia Sea,

23032.6 .5 - the peculiar meteorology and opposing wave conditions of the Red Sea,

23042.6 .6 - the muddy bottom off the Kerala coast of India. 


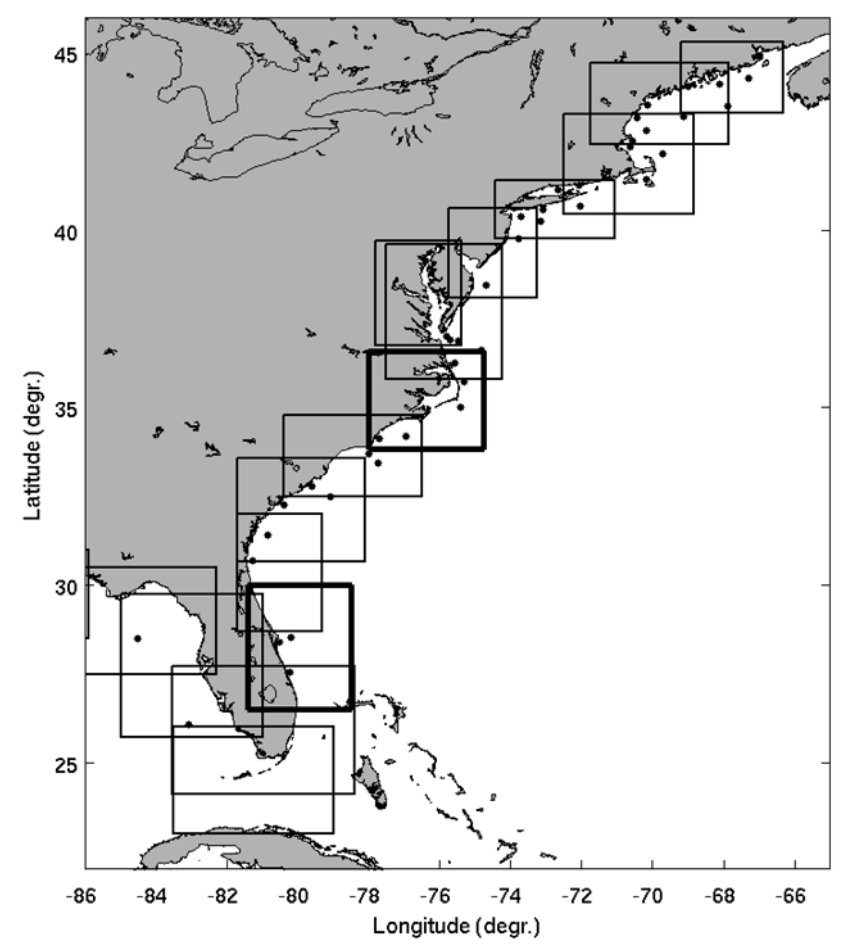

2309 Figure 2.15 - Distribution of the various sub-zones in the modulational approach to wave forecast 2310 on the US East coast.

2312 The US coast, facing two oceans and with an often articulated coastline, is a classic example of how 2313 to combine large scale and inner/coastal seas approaches into a sensible and effective working 2314 machine. While the two different scales naturally involve different spatial resolutions, it is also 2315 obvious that the extent and different characteristics of the coastline imply a modulational approach 2316 and, to be effective, a distribution of the responsibilities. The solution is shown for the Atlantic 2317 coast in Figure 2.15 (NCEP). Granted the global ocean model and the availability of its results as 2318 boundary conditions, the coastline is subdivided into a number of sub-zones, each one with a 2319 different model unit and under the responsibility of a local office. For operational purposes NOAA 2320 currently uses implementations of the WAVEWATCH III (Tolman, 1991) and SWAN (Booij et al., 2321 1999) models driven by atmospheric fields provided by its coastal Weather Forecast Offices 2322 (WFOs). A SWAN-based modelling system, the Nearshore Wave Prediction System (Van der 2323 Westhuysen et al, 2013, 2014), provides downscaled guidance for open-ocean coastal areas relative 2324 to the Global WAVEWATCH III model. Parallel to this, a high-resolution WAVEWATCH III system 2325 , the Great Lakes Waves Forecasting System (GLW), is used for operational forecasts in the North 2326 American Great Lakes (Alves et al., 2014).

2328 The challenges of the complex orography and associated flow fields in these coastal areas are 
addressed by allowing coastal WFOs to force the wave model at these scales with forecasterconsensus winds, as opposed to one or more raw atmospheric models. Those forecaster-consensus winds are compiled from ensembles of individual atmospheric models, and adjusted for biases or other known deficiencies using available observations. As such, they constitute the official atmospheric forecast in these complex regions.

Due to the routine human intervention in the forcing applied, the NWPS runs are conducted in an on-demand fashion, with forecasters from 36 WFOs triggering the runs over their individual domains, which are computed on NOAA's operational supercomputer. The GLW system is forecastdriven, whereby marine forecasters from 11 WFOs provide consensus winds for their areas of responsibility. Wind fields are modified by the forecasters and are stitched into a coherent wind field covering the wave model domain (Figure 2.15), which are sent to NOAA's operational computer on an hourly basis. GLW model runs are made in four daily cycles using the latest consensus wind fields.

The operational NWPS applies SWAN v40.81, using the wind input and white-capping dissipation expressions recommended by Rogers et al. (2003), and default settings for the remaining shallow water physics. Wave boundary conditions are taken from NOAA's Global WAVEWATCH III model discussed in Chawla et al. (2013). This global model, driven by NOAA's Global Forecast System (GFS) atmospheric model, features a two-way nesting with a resolution of 4 nmi over the US shelf seas. NWPS downscales this coastal resolution to nominally $1 \mathrm{nmi}$, and where required by local features to $500 \mathrm{~m}$ or less, in deterministic forecasts out to $102 \mathrm{~h}$. At present, this is achieved with a regular grid for each WFO domain, followed by smaller-scale nesting, to be replaced in the future by variable-resolution unstructured meshes.

At these resolutions, it becomes necessary to include the interaction of wind waves with coastal currents. Closer to the shore, accurate estimates of the total water depth including tides and storm surge, and its effect on the wave evolution, become essential. This has been illustrated in recent events such as superstorm Sandy (2012) and Winter Storm Jonas (2016), which featured large coastal surges, and significant sustained damage due to wave action. Under these conditions, the greater water depth due to the combined effect of tides and surge allows larger waves to reach the coast. These effects and the related modelling approach are described in Section 3.1.

A direct action of the local WFOs is obviously more useful in now- or very short term fore-cast, the 
2363 system being active four times per day. In the medium range the local forecast is fully dependent on 2364 the global one and following nesting, with all the related implications. This is clearly shown by the 2365 performance statistics versus buoy data. Table 2.1. summarizes the performance of the 2366 NOAA/NCEP multiple system against the significant wave heights measured by 50 NDBC coastal 2367 buoys in the Gulf of Mexico and along the US East coast. 24, 48, 72 and 96 hour forecast horizons 2368 are considered. It is quite remarkable that the limited bias, between -4 and $6 \%$, and the best-fit slope 2369 do not change with increasing range.

One example of the advantage of the NWPS was given by the passing at Duck (N.C., see Figure 2.1) of hurricane Joaquin which traversed the North Atlantic between 27 September and 7 October 2015. Three buoys were locally in operation at different distance from the coast, respectively on $47.6 \mathrm{~m}$ (the most offshore one), $26 \mathrm{~m}$, and $17.4 \mathrm{~m}$ depth. While the differences between the global wave model and the local NWPS were practically absent offshore and limited at the intermediate buoy, they were dramatic at the close to shore one, the two models providing respectively 2 and 4.5 m maximum significant wave height, the latter close to the actual measured value.

Although facing similar challenges, the GLW runs as a separate system at NCEP, focusing on the particular environment given by the Great Lakes of North America. The complex meteorological scenarios that develop in this area forced the local development of the GLW already in 2004, a precursor of the then general system for all the US coasts. The local conditions, with mid-latitude cyclones, artic air mass intrusions, and organized intense convective systems, has forced an early shift to a higher resolution (from 12 to $4 \mathrm{~km}$ ) of the forcing winds, with a marked improvement of the final results. GLW uses WAVEWATCH III on a $2.5 \mathrm{~km}$ curvilinear spatial grid, with an expected shift to an unstructured grid in 2017.

\subsection{2 _ The Adriatic Sea and the enhancement of wind speed}

In Section 2.1 we have hinted to the need, but also to the difficulty, of having sufficiently correct winds in an enclosed sea. When passing from land to sea surface, winds experience a suddenly decreased surface drag. The reason is that, although the local wave age is often very young, nevertheless the surface drag is substantially lower than on land. Under the action of the overall meteorological situation and in correspondence to the high above geostrophic wind, surface wind tends to a new, higher dynamical equilibrium. A new marine surface boundary layer is developed, and $\mathrm{U}_{10}$ keeps increasing while blowing toward offshore till reaching the new equilibrium 
condition. The time, hence distance, required vary with the situation, always in the order of a few tens of kilometres. Not so a modelled wind. Partly for the not fully correct representation of the physics involved, much because of spatial resolution, the time and space required by a modelled wind to reach a new equilibrium are larger. The practical consequence, relevant for wave modelling in these areas, is that the modelled $\mathrm{U}_{10}$ is underestimated in the coastal area till when and where the model achieves the new equilibrium. Of course this has practical consequences. The first one is that for an offshore blowing wind the locally generated wave conditions are underestimated for a longer extent than for wind (wave growth takes time).This explanation sorted out a long standing problem concerning wind and wave modelling on the US East coast. As measured by NOAA buoys, moored till many tens of (up to 200) kilometres offshore, modelled wind speeds appeared correct, but not so the wave heights, always too low (with an offshore blowing wind of course, there a common situation). The explanation is what said above, and the problem has been progressively attenuated with the progressive increase of spatial resolution of the meteorological models.

Moving from the oceanic coastal waters to the enclosed ones, it is clear that, depending on the size of the basin, wind from all the directions can be affected, leading to a permanent underestimate. When the MEDATLAS Group (see Sclavo et al., 2002) produced the Mediterranean atlas of the

2414 local wind and waves using the ECMWF archive, they first enhanced the modelled data using 2415 distributed altimeter data. Consistently with the dominant direction of the storms from the North2416 West quadrant moving towards the South-East sector, the enhancement factors (see Figure 2.2, 2417 panel a) were higher close to Spain and France, progressively decreasing and approaching unity 2418 getting close to the African coast.

A more complete case is the Adriatic Sea (see panel b), practically enclosed, whose dimensions, $200 \times 700 \mathrm{~km}$, make it deal with the wind underestimate for practically all the possible conditions.

2422 Indeed Cavaleri and Bertotti, starting with their 1997 paper, using the ECMWF wind for the local 2423 wave forecast system (Bertotti et al., 2011), have been forced to enhance the surface wind speed to 2424 get the correct wind (versus scatterometers) and wave (versus altimeters and buoys) results. While 2425 on a more approximate approach a uniform correction factor could be used, a further improvement could be reached using coefficients area (north, central, south) and quadrant $\left(0^{\circ}-90^{\circ}-180^{\circ}-270^{\circ}\right.$ $360^{\circ}$ ) dependent. This was associated to the different longitudinal and transversal dimensions (700 2428 vs $200 \mathrm{~km}$ ), but also to the dominant mountain ridges that characterize both the long sides of the 2429 basin. 
2431 The progressive increase of resolution of the ECMWF model has led to a parallel decrease of the 2432 "fetch" required by wind to reach its sea equilibrium, hence correspondingly of the wind 2433 enhancement factors from the original 1.50 (for T213, with nominal $90 \mathrm{~km}$ resolution) till the 2434 present 1.16 (for TCo1279, with $9 \mathrm{~km}$ resolution).

Although the local quantified experience is mainly based on ECMWF winds, parallel tests have shown this to be, possibly to a different extent, a feature of all the meteorological models.

\subsection{3 - Wave forecast for the Rotterdam channel}

2441 Rotterdam harbour is the busiest one in Europe and competing to be the most one in the world. 2442 Located at the south end of the North Sea, on the Dutch coast, it is not in principle in the most 2443 favourable position. The progressively shallowing North Sea ends at the Dutch coast with a very 2444 limited depth, largely unsuitable for the present large oil and container ships. The problem has been 2445 solved dredging a $76 \mathrm{~km}$ long channel, now $800 \mathrm{~m}$ wide, $26 \mathrm{~m}$ deep, till where a similar depth is 2446 naturally found. Figure 2.16 shows a scheme of the channel. Of course the sandy bottom and the sea 2447 storms imply an almost permanent dredging to keep the channel in the desired conditions. We focus 2448 here on the local wave forecast system.

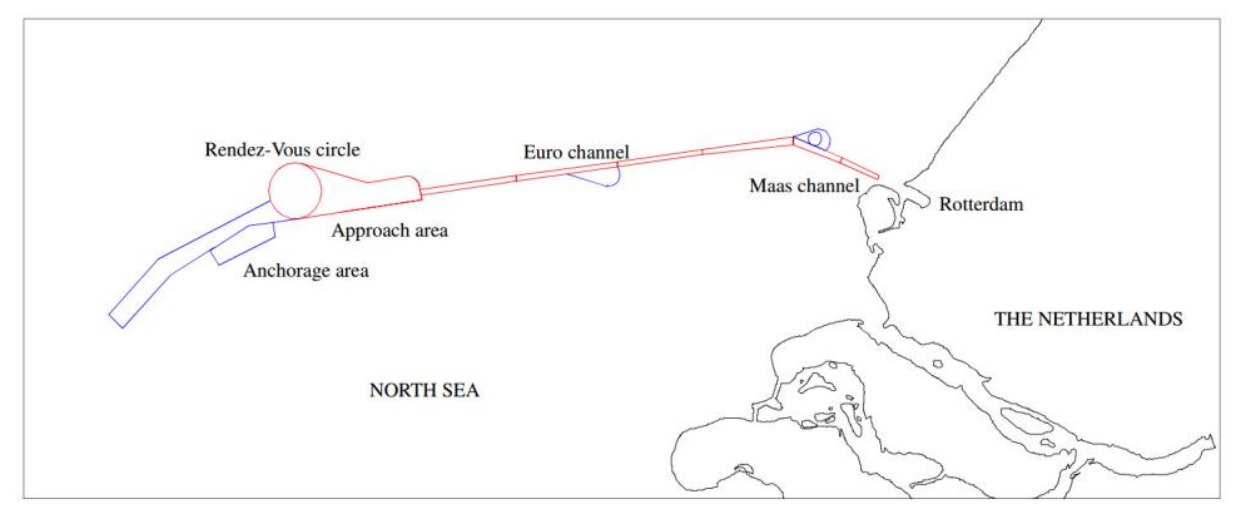

2450 Figure 2.16 - Structure of the channel leading to the Rotterdam (Netherlands) harbour in the southern North Sea

2453 Given the cost of maintaining the channel geometry, its depth is not larger than necessary. One 2454 meter clearance is accepted between the bottom and the keel of the ships. This implies that, beside 2455 tide, also the wave conditions are critical for making the passage of a big ship possible or not. Because, once a ship has entered the channel, it takes a few hours to reach the other end, given also the consequences of an accident, it is mandatory to have a reliable estimate of the incoming wave 
conditions. Mandatory for the next few hours, its forecast for the days ahead allows an optimization of the future activity.

With these needs in mind, a tidal and wave forecast system has been set up (Gautier and Caires, 2015). The system is based on the SWAN model (Booij et al., 1999) with two different grids. The first, coarse one, called SWAN-DCSM, covers a large area $\left(-12^{\circ}\right.$ to $+9^{\circ} \mathrm{E}, 48^{\circ}$ to $\left.64^{\circ} \mathrm{N}\right)$ and computes boundary conditions for the detailed nested model domain. The resolution is $1 / 20^{\circ} \times 1 / 30^{\circ}$ (which is circa $3.6 \times 3.6 \mathrm{~km}$ ). The nested model, SWAN-ZUNO, has a curvilinear grid with resolution varying from $2 \mathrm{~km}$ offshore to circa $200 \mathrm{~m}$ close to the coast. HIRLAM wind fields from the Dutch meteorological institute (KNMI) and large scale boundary conditions from the ECMWF global model are used. Water level and current are similarly forecast.

For the ship motion, in particular heave and pitch, while in the channel, there is special interest in the low frequency wave energy $(0.03-0.1 \mathrm{~Hz})$. Therefore, beside the standard integral parameters $\mathrm{H}_{\mathrm{m} 0}$ and $\mathrm{T}_{\mathrm{m}-1,0}$, the so-called low frequency wave height $\mathrm{H}_{\mathrm{E} 10}$ is considered, defined as the wave height corresponding to the energy in the $0.03-0.1 \mathrm{~Hz}$ range.
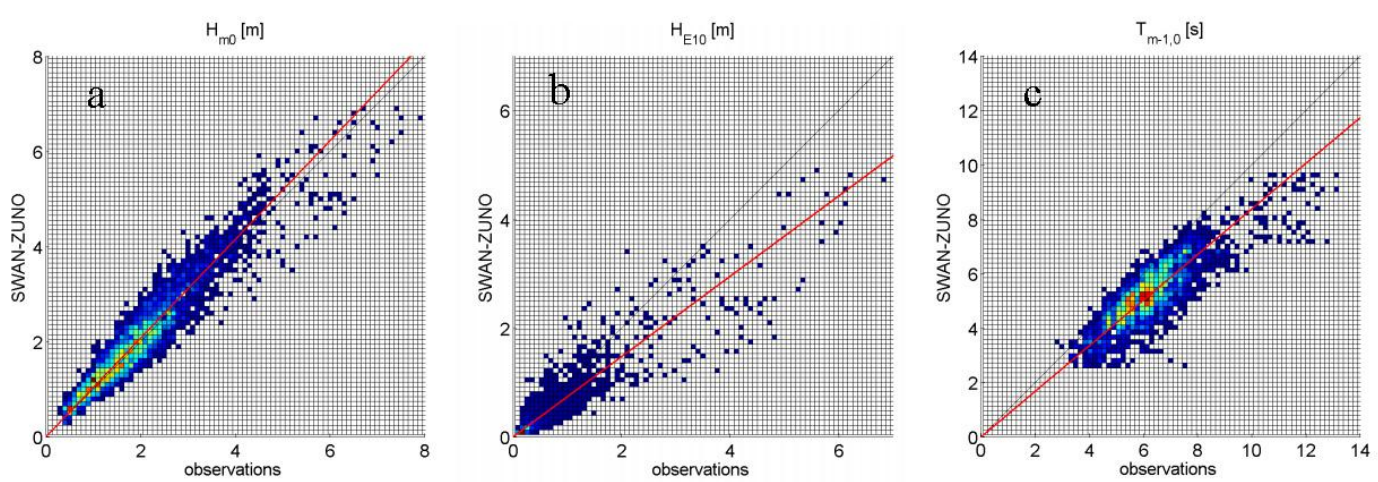

Figure 2.17 - Performance of the wave forecast system for the Rotterdam channel (see Figure 2.16). Model comparison vs buoy. a) significant wave height, b) $\mathrm{H}_{\mathrm{E} 10}, \mathrm{c}$ ) mean period $\mathrm{T}_{\mathrm{m}-10}$.

Figure 2.17 shows the performance of the above model system versus measured data. While the results are satisfactory for $\mathrm{H}_{\mathrm{m} 0}$, it is evident there is, beside the large scatter, a substantial underestimate of $\mathrm{H}_{\mathrm{E} 10}$ and, although at a smaller extent, of also the mean period. Of course the last two results are related because an underestimate of $T_{m-1,0}$ implies that on average the model places the (on average) right amount of energy too much toward the higher frequencies. Unavoidably this leads to an underestimate of the energy in the $0.03-0.1 \mathrm{~Hz}$ range, hence of $\mathrm{H}_{\mathrm{E} 10}$. The reasons for this are still unknown and, apart from specific tuning, research is on the way to find the culprit (or culprits) for this. The possibilities range from the large scale (wind and wave input, wave model, 
etc.) to the very local ones. For instance, zooming on the local scale, Groeneweg et al (2015) have pointed out how, considering the interaction of the waves with the channel, the absence of various sub- and super-harmonic interactions in SWAN causes an unrealistic amount of energy to be trapped on the channel slopes owing to wave refraction.

On more general terms it has been pointed out that the $\mathrm{H}_{\mathrm{E} 10}$ concept, conceived 20 or 30 years ago, at a time when first hand solutions were required, should be abandoned in favour of a more rigorous solution. Granted that the full 2D spectrum is available, a specific estimate should be provided for each vessel on the base of the its response function. Of course we still have the general model energy shift towards higher frequency, but in a busy and economical world where accident probabilities and ensemble consequences are carefully evaluated, this is clearly the way to go.

\subsection{4 - The fractal coastline of the Bothnia Sea}

Few places in the world, if any, can be compared as complexity of the local geometry and bathymetry to the Bothnia Sea in the central part of the Baltic Sea. Figure 2.18 shows a progressive zooming on the area. Even at the $100 \mathrm{~m}$ scale it is impossible to resolve all the minor islands and tiny rocks that emerge from the surface. The submerged part and the convoluted shape of the shoals add to the complexity of the archipelago. There is no doubt that wave modeling here is a challenge because the scale of the relevant processes, as refraction and depth induced breaking, is beyond the practical resolution of even experimental runs.

The Finnish Meteorological Institute has been very active in improving the wind and wave modeling in the area, both for navigation purposes (ship routes do pass through the archipelago) as also for estimating the conditions out of, but relatively close to, the archipelago. Ten different detailed grids were generated based on coastal nautical charts with horizontal resolutions of 0.1 and $0.5 \mathrm{nmi}$, also using different methods. Running the WAM model with locally produced high resolution winds, Pettersson et al. (2014) found that the minimal, $0.1 \mathrm{nmi}$, resolution was essential even only to obtain meaningful results. However, even at this resolution problems still exist. Model 

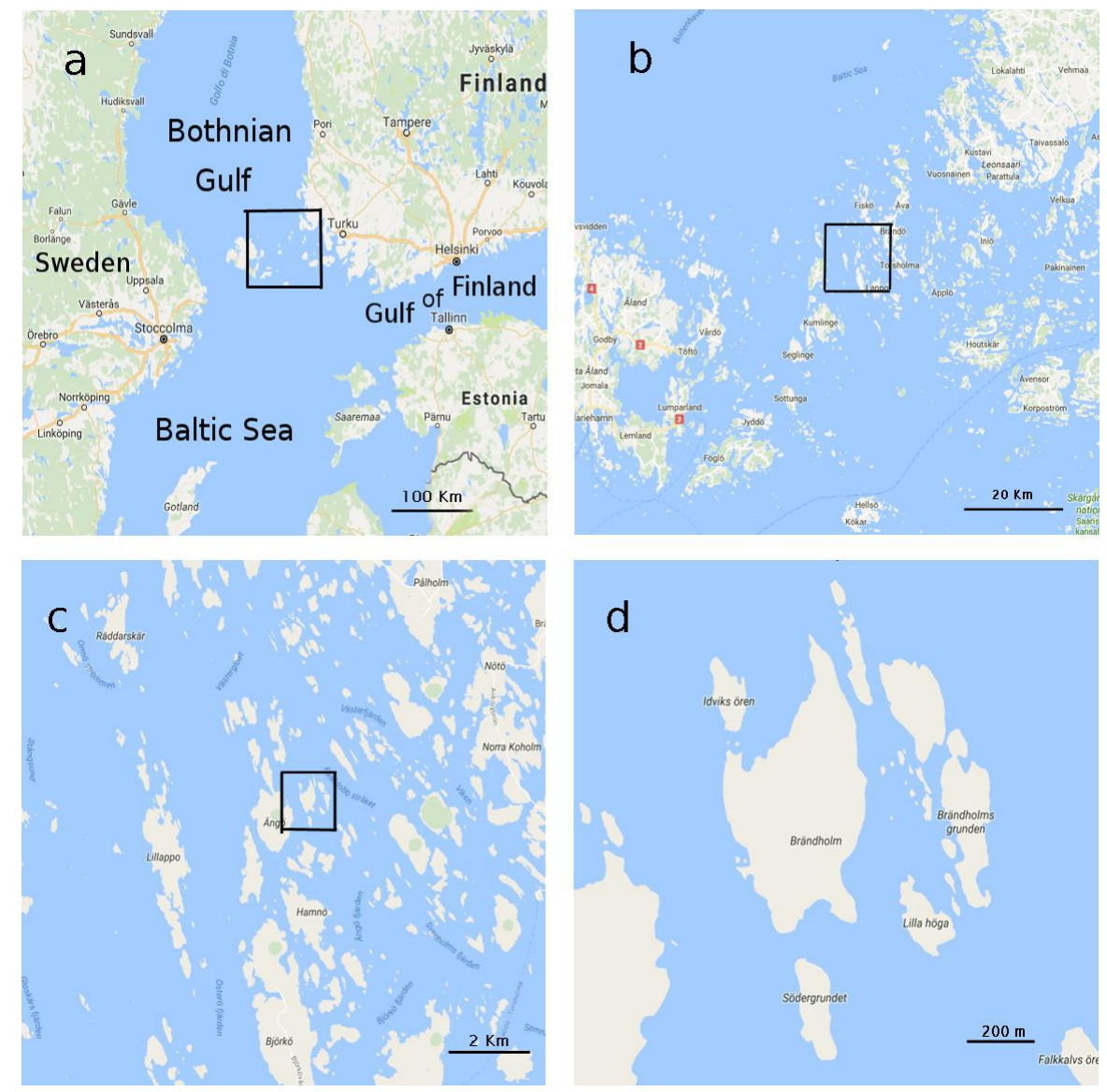

2516 Figure 2.18 - Progressive zooming on the archipelago in the Baltic Sea. Each panel is, in the order, 2517 the enlargement of the little square in the previous panel.

2519 data have been compared with the data (wind and wave) recorded at the Utö buoy, located slightly 2520 south of the archipelago. The results are in Figure 2.19. The blue dots refer to southerly wind 2521 conditions (hence blowing from the open water towards the archipelago). The red ones refer to the 2522 opposite direction. It is clear that, granted the usual and expected scatter, the wind has no particular 2523 problem. However, while the South coming waves are slightly underestimated, there is a substantial 2524 overestimate of the waves coming from the archipelago. This suggests that the model does not 2525 succeed in reproducing the true conditions among, and due to, the islands. Possible reasons are a 2526 still insufficient resolution or the complexity of the large and small scale generation and dissipation 2527 that take place among and along the islands. One wonders if a solution, however pragmatic, is 2528 possible. This will be further discussed in the final Chapter 5. 

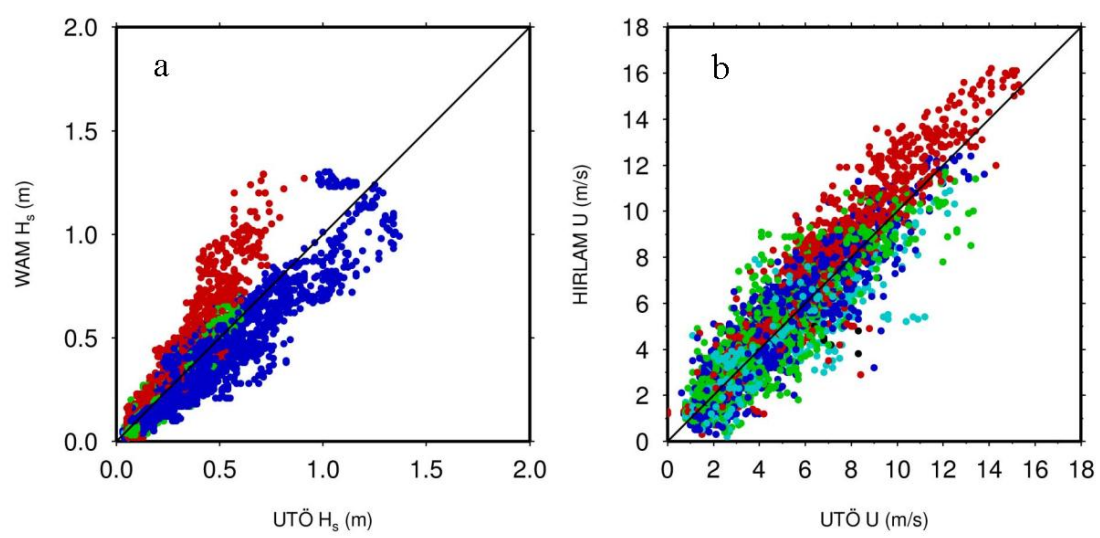

2531 Figure 2.19 - Comparison between model and Utö buoy data. Location just South of the 2532 archipelago shown in Figure 2.18. a) significant wave height, blue North going, red South going, b) 2533 corresponding wind speeds.

2.6.5 - The peculiar meteorological and wave conditions of the Red Sea

The Red Sea is the narrow $(200 \mathrm{~km})$ and long $(2200 \mathrm{~km})$ almost closed basin between Africa and the Arabian peninsula. Its borderline can be seen in Figure 2.21. The orography is pronounced on both the sides, with cutting valleys aiming directly to the sea. Two opposite wind regimes dominate the situation. The periodic storms moving to East in the Eastern Mediterranean (just off the northern part of the map) lead to relatively cold inflows from the North that move than South channelled by the border orography. Alternatively during the winter months the East-North-East monsoon in the gulf of Aden (just out of the lower-right corner of the map) forces strong winds through the Bab-elMandeb strait (the only opening of the Red Sea). These winds are then again channelled by orography in the longitudinal direction of the basin, in so doing being exactly opposite to the ones coming from the Mediterranean Sea. When, as it happens in winter, both the conditions are present, a peculiar, rather unique situation arises in the basin. The two winds, colder from the North, warm 2548 from the South, meet somewhere forcing the South coming lighter air mass (see Figure 2.20) to 2549 raise above the incoming northern air. This gives raise to local clouds and drizzles in the middle of an otherwise fully sunny area. The corresponding wind (panel a) and wave (panel b) conditions are shown in Figure 2.21. Panel c reproduces the distribution along the Red Sea of the energy of the two wave systems, the red one moving to South, hence decreasing in this direction, the black one to the North. The wave spectra at three separate positions (see also their geographical coordinates in 2554 Figure 2.22) provide a clear evidence of the contemporary presence of the two systems. 


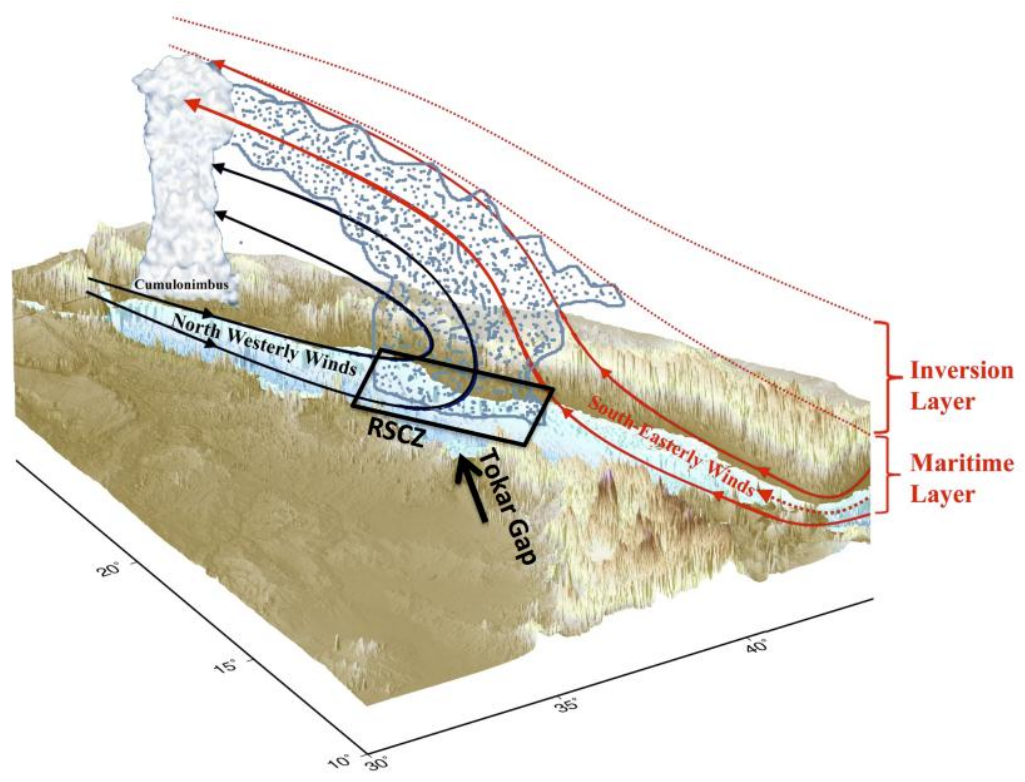

2557 Figure 2.20 - Red Sea. Encounter between the cold north-westerly wind and the warm south2558 easterly one. The scale is provided by the geographical coordinates.

a)

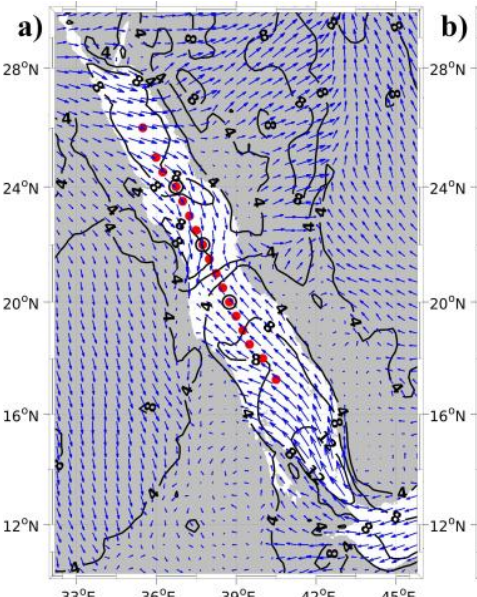

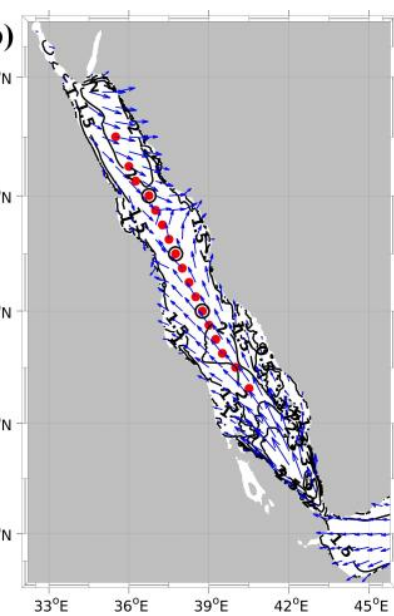

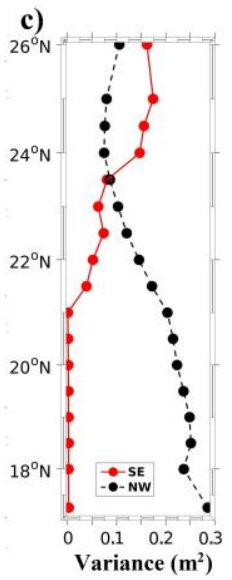

Figure 2.21 - Red Sea. a) Surface wind speeds corresponding to the situation in Figure 2.20. Isotachs at $4 \mathrm{~ms}^{-1}$ interval. b) significant wave height distribution corresponding to the situation in panel a. Isolines at $0.5 \mathrm{~m}$ interval. c) distribution of the wave energy (at the dots in panel b) for the two North and South going systems. Black moving to North-West, red to South-East.

From the wave modelling point of view the unique situation is the one of two opposite winds blowing against each other, hence two opposite wind seas clashing together. The physical aspect of interest is that in this situation many of the implicit assumptions at the base of the wave model physics are no longer true. In the standard situation of a generative sea, wind and waves move more or less in the same direction, and, with some theoretical background and a sufficient level of tuning, 
2572 we have suitable formulas for energy input by wind and dissipation by white-capping. However, in 2573 the described situation the physics is, partly at least, different. This forced Langodan et al. (2015) to

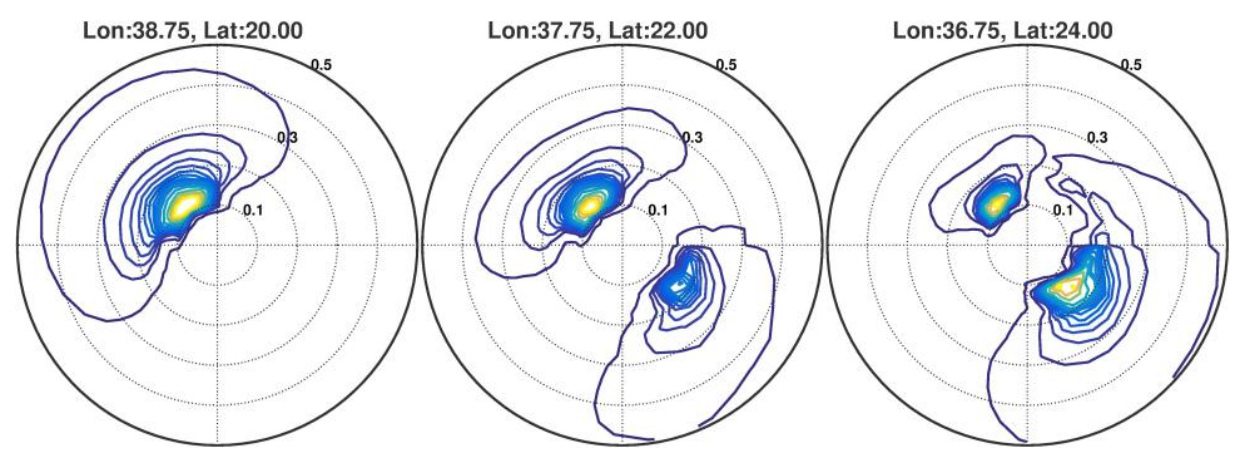

2576 Figure 2.22 - 2D wave spectra corresponding to three points in panel a (see coordinates). Note the presence of the two opposite wave systems.

propose, in what they define "a preliminary crude attempt", and use a modified version of the two corresponding approaches. Given $E_{A}$ and $E_{B}$ as the energy of the two opposing systems, the modified source functions appear as

$\mathrm{S}_{\text {inA }}=[\ldots \ldots \ldots]\left(1-\alpha\left(\mathrm{E}_{\mathrm{B}} / \mathrm{E}_{\mathrm{A}}\right)\left(\mathrm{L}_{\text {short }} / \mathrm{L}_{\text {long }}\right)\right)$

$\mathrm{S}_{\mathrm{dis}}=[\ldots \ldots . .].\left(1+\beta\left(\mathrm{E}_{\mathrm{B}} / \mathrm{E}_{\mathrm{A}}\right)\left(\mathrm{L}_{\text {short }} / \mathrm{L}_{\text {long }}\right)\right)$

where [...] indicate the presently used source functions. $\mathrm{L}_{\text {short }}$ and $\mathrm{L}_{\mathrm{long}}$ are the wavelength of the two systems, the shorter and longer one respectively, independently of which system the source function refers to. Langodan et al. (2015) report a marked improvement of the quality of the results using $\alpha=0.08$ and $\beta=0.20$, the different values reflecting the also physical perception that whitecapping is the most affected process in the described situation. Of course problems exist also for the DIA approximation. Conceived for and sufficiently fitting the classical case of a single generative sea, obviously, as also shown in the cited paper, it cannot cope with the "encounter" situation. We stress that this "encounter" is substantially different from the common case in the ocean of a wind sea superimposed to a background swell, this typically with different (lower) frequency and direction. In the Red Sea case the two systems have more similar, albeit varying along the respective fetch, frequencies. The "preliminary crude attempt" of Langodan et al. (2015) is only a patch, although physically in the right direction, hinting to the fact that the physics we presently use in wave modelling is based on idealized conditions that often (we wonder, e.g., about the much simpler case of a wind sea plus a non-small swell) do not properly represent the truth of nature. 
26012.6 .6 - The muddy bottom off the Kerala coast of India

2603 Kerala is the southernmost state on the west coast of India. Wave climate is dominated by the long swells coming from the Southern Ocean and by the southwest monsoon waves (predominantly wind seas). A peculiar phenomenon existing off Kerala is the non-permanent presence of a thick layer of viscous mud (mud-banks) in an extended shallow area just off the coast. Locally known as 'Chakara', the dimensions of the mud-banks are not yet known. The mud, whose origin and transport have not yet been fully determined, has the peculiar characteristic of leading to an area of relatively calm zone (soon to be quantified); off the periphery of this calm zone, rough conditions prevail because of the southwest monsoon waves (June-Sept). During this time the significant wave height off the periphery of this mud-bank would be up to 3 to 4 meter, with the highest values 2612 frequently above 4 these figures. On the contrary in the mudbank area the wave heights are highly 2613 attenuated. Figure 2.23 shows the $\mathrm{H}_{\mathrm{s}}$ recorded by two Waverider buoys at two water depths $(15 \mathrm{~m}$ 2614 and $7 \mathrm{~m}$ ) off Alleppey (Kerala). The two buoys are located at $10 \mathrm{~km}$ and $5 \mathrm{~km}$ off the coast, 2615 respectively. The mudbank and its periphery are known for their very high biological productivity, 2616 leading to a flourishing fishing activity. In practice, the mud is an important factor for, and a clock 2617 of, the local economy.

The fact that mud in shallow water attenuates waves is not new. Spectacular attenuations have been reported in the Gulf of Mexico when large waves come across narrow gulleys with a thick layer of viscous mud. Forristall and Reece (1985) and Forristall et al (1990) made an extensive study of the situation. When the mud is distributed along elongated gullies, the effect is in a way rather local. On the contrary, along the Kerala coast the mud is distributed over a large area, leading, where present, to a progressive attenuation of the waves while approaching the coast. An excellent aerial view of mud accumulation has been provided by Holland et al. (2009), and a summary of the mud attenuation related studies is found in Komen et al. (1994, 169-171). The CSIR-NIO (Goa, India) initiated a multi-disciplinary oceanographic program, "Alleppey Mud-bank Process Studies (AMPS)" in 2014 (still continuing) to study the various aspects of Alleppey mud-banks, including also a high resolution wave forecast system. An extensive description of the Alleppey mud-banks and its local phenomenology is provided by Samiksha et al. (2015) and Shinu et al. (2017). Although a number of studies/hypotheses have been conducted/proposed for the origin, the 
and current related in association with the southwest monsoon, not enough data are available to predict with sufficient accuracy the formation of the mud-banks.

2635
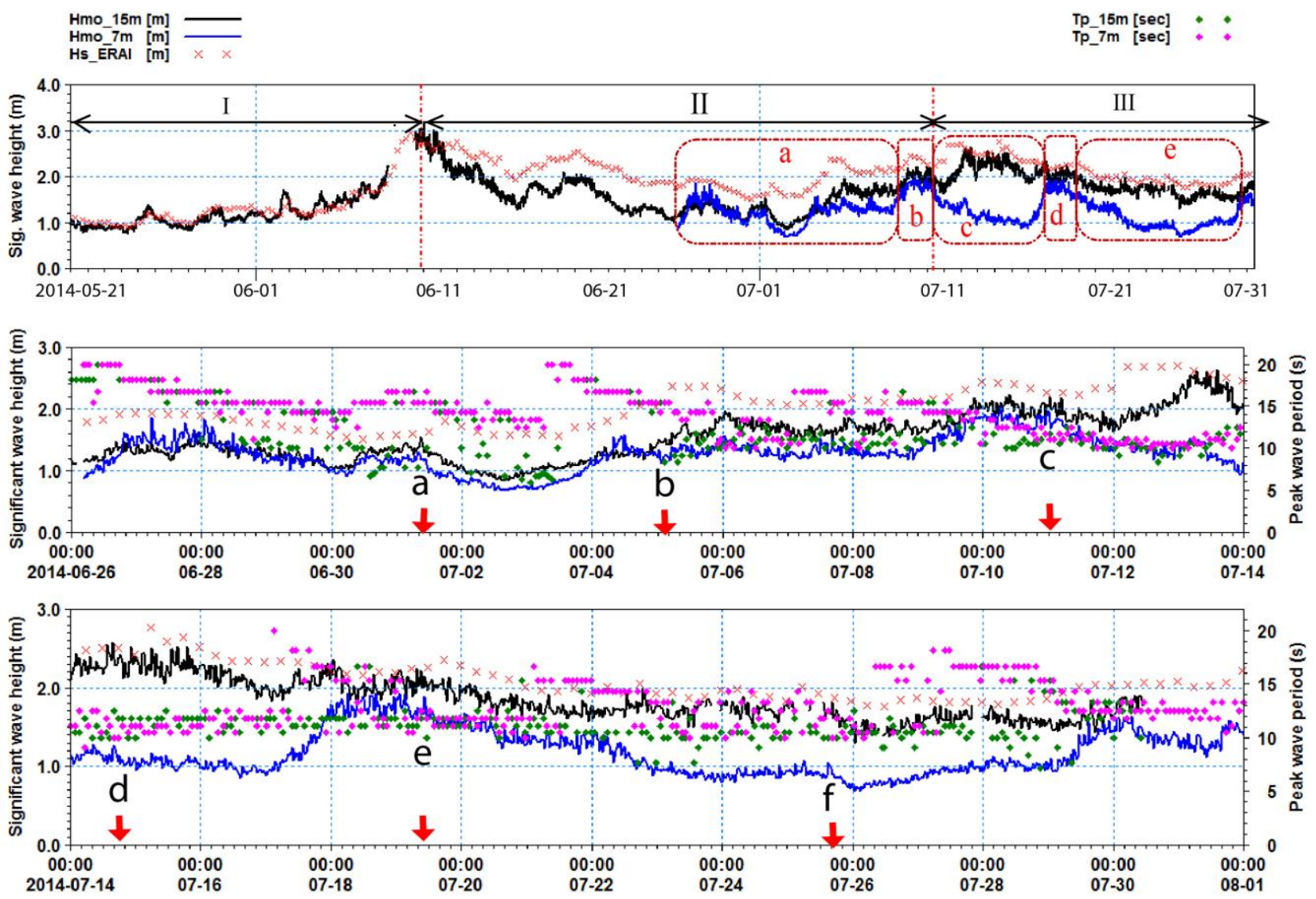

Figure 2.23. Significant wave heights recorded off Alleppey (Kerala, India) from two buoys at $15 \mathrm{~m}$ (black) and $7 \mathrm{~m}$ depth (blue) water depths. Red line shows the corresponding ERA-Interim values. The dots (pink and green) show peak wave periods at the two locations. The upper panel is $\mathrm{H}_{\mathrm{s}}$ during May to July 2014. The right part (II \& III) is expanded in the central and lower panels, wherein the a to f letters refers to the time of the spectra in Figure 2.24. Days are given as yyyymm-dd. Please disregard the a to e letters in the top panel, written there for a different purpose not part of this paper. We apologize for the confusion. (derived from Samiksha, 2015, and Shinu et al. 2644 2017).

The two above cited studies provide a detailed description of the wave attenuation due to mudbanks. The wave heights recorded at the two cited buoys are compared are compared to the corresponding estimates derived from ERA-Interim (Dee et al., 2011) as well as model results. Here, we reproduce two figures taken from the above work, that offer a spectrum of the possible situations. The upper panel of Figure 23 (derived from the above works) shows the $\mathrm{H}_{\mathrm{s}}$ time series from the two buoys (the offshore buoy is denoted by B, black, and the onshore buoy by C, blue) 
measured during May to July 2014, and the corresponding (close to the offshore buoy) ERA2653 Interim $\mathrm{H}_{\mathrm{s}}$ (red, A). Sections II and III (Figure 2.23, upper panel) are then expanded in the 2654 respectively central and lower panels, wherein peak wave periods are also provided (but not 2655 discussed). In these two panels six specific times are identified (a to $f$ ) and the corresponding spectra from the two buoys are shown in Figure 2.24. We offer only a qualitative analysis that however suffices to make evident the difficulty of local wave modelling.

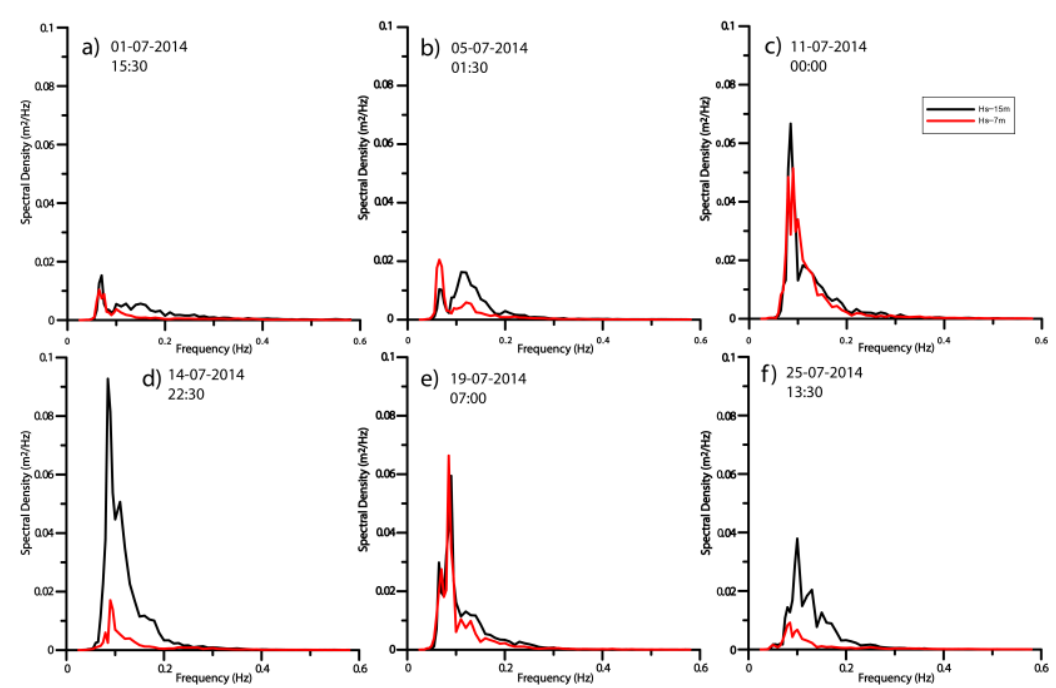

2660 Figure 2.24 - Wave spectra at 15 and $7 \mathrm{~m}$ water depth locations, at the six times (a to f) shown in the central and lower panels of Figure 2.23. Days are given as dd-mm-yyyy (a different format from 2662 Figure 2.23).

Till the first $3 \mathrm{~m}$ peak around the middle of May, (the) A, B, C $\left(\mathrm{H}_{\mathrm{s}}\right)$ coincide, an indication (of the quality of ERA-Interim data, but for our present purposes) that mud has not yet reached the area. After this episode $\mathrm{A}>\mathrm{B}=\mathrm{C}$, (suggesting that) mud has reached the area, but still offshore $\mathrm{B}$, hence the limited differences (only in the higher frequency range) between the two spectra. Further in section II, time $b$, mud is also between $B$ and $C$ (hence $A>B>C$ ). The two spectra show a more marked attenuation at $C$. At $c$ the mud is back offshore $B$, hence $A>B=C$, and the two spectra practically coincide. The situation changes completely in section III (lower panel) when the mud is now shoreward of B. At time $\mathrm{d}$ it is between $\mathrm{B}$ and $\mathrm{C}$. Note the attenuation of the corresponding $\mathrm{C}$ spectrum with respect to $B$. At e most of the mud is shoreward of even $C$, so that $A \approx B \approx C$ and the spectra are again the same. At time $\mathrm{f}$ the mud is moving towards offshore, now between $\mathrm{B}$ and $\mathrm{C}$, so that $\mathrm{A}=\mathrm{B}>\mathrm{C}$ with a highly reduced spectrum at $\mathrm{C}$. To close the cycle note how at the end of III the two buoys are again the same, but both $\mathrm{B}, \mathrm{C}<\mathrm{A}$, i.e. the mud is now offshore $\mathrm{B}$. 
2677 In other areas of the world, where present, the mud-banks are permanent (the Amazon being a 2678 classical example), generally at the mouth of rivers, and the formation mechanism is clear. It may 2679 be noted that in case of mud-banks off Alleppey, there is no presence of a river. Off Alleppey 2680 (where by the way no river exit is present)) and the Kerala coast in general, the mud is moving in 2681 with the monsoon waves and currents. With such variable conditions, it is difficult to 2682 predict/forecast waves accurately, when the mud distribution of this area is not known. 2683 2684 2685 


\section{3 - Interactions and coupling}

where we describe the physics and the applications of fully coupled systems, first waves with currents, and then with also the atmosphere

In the previous chapter we have done a general, occasionally deeper, survey of the meteorology and wind waves that characterize the enclosed and coastal seas. We have highlighted the main differences with respect to how the same processes act and where they lead in the great open oceans. Oceans do cover most of the earth surface, but large part of the human population lives along the coasts, and most of our economic activity is concentrated in this area.

The shorter wavelengths and the younger and steeper waves that characterize the inner seas make waves more sensitive to the interaction with currents and also with the atmosphere. Some of the physics involved is similar, but often enhanced, with respect to the open oceans. In particular the steeper waves and the shallow water processes imply a higher level of non-linearity in the system. Of course this makes the analysis and, more so, the forecast more "delicate", in the sense that limited differences in the input information may lead to substantial ones in the final results.

Different processes arise, typically in shallow water. Indeed the costal time scale where waves and current interact can be of the order of 10 or $100 \mathrm{~m}$. All this poses new challenges to the corresponding modeling, both as physics and numerics. While in the previous chapter we have considered the single aspects of the problem, e.g. the meteorological and waves ones, each one on its own, it is now time to go closer to the true world discussing the thin layers of air and water that surrounds our planet as a single unit and exploring what this implies. However, given the purpose of this paper, we will not take the grand view of the climate. Rather, our, perhaps biased, focus will be mainly on the inner seas, although some general discussion on the physics involved will imply describing the parallel processes in the wider open seas. For a progressive approach to the problem we will first deal (Section 3.1) with the wave-current interactions, that we will then expand in

2713 Section 3.2 with some consideration of the role of atmosphere in the coupling of the full system.

jane.m.smith@usace.army.mil

\subsection{Wave-circulation interactions}


with the physics of the interactions between waves and current and illustrative examples on the various aspects of interaction

Interaction between wind-generated surface gravity waves and current represents one of the important driving forces in coastal and offshore areas. Waves and circulation (partly the latter) are dominantly governed by the same driving factor (the atmospheric wind) and propagate in the same medium (the oceanic water). Waves and currents form a complex system which has usually been discussed assuming distinctly the influence of waves on hydrodynamics and the influence of currents on waves, a reductionist approach that, despite having merits, should be overcome by an integrated holistic approach. This broad topic is usually referred to as Wave-Current Interactions (WCI) which traces back to the pioneering work of Longuet-Higgins and Stewart (1960, 1961).

Before discussing the problems related to practical applications, it is useful to frame the problem from a very general point of view. From this perspective the overall problem of wave-current interactions could be dealt with in a complete way solving the non-linear shallow-water equations including the non-hydrostatic picture of the local environment. The basic ideas can be found, among others, in Zijlema and Stelling (2008) and Smit et al. (2014). However rigorous in principle, this approach is not pursuable in practical applications for various reasons ranging from the availability of all the necessary information to, most of all, the enormous volume of the implied computer resources. Hence a different approach is required.

To make the problem manageable, surface gravity waves are usually averaged out of numerical circulation models by integrating the governing equations of continuity and momentum over the time scale of the short wave motions. Circulation varies slowly in space and time at a scale that for most applications is large compared with typical wave lengths and periods. In spite of the separation in frequency space (minutes to weeks for circulation versus 1-30 sec for wind generated waves), the interactions between circulation and waves have first-order impacts on both processes in key coastal locations such as coastal inlets, channels, and surf zones.

The general circulation near the coast and in estuaries or bays affects surface gravity waves through refraction, shoaling, and breaking. The latter occurs where the currents are strong (generally in the range of $1 \mathrm{~m} / \mathrm{s}$ or more) or due to wave-water depth interactions, where tides or storm surges significantly alter the water depth (Jonsson,1990). In turn waves impact the circulation through momentum transfers that drive currents, including Stokes-Coriolis force and wave setup (Longuet- 
2757 Higgins and Stewart, 1960, 1961, 1962, 1964; Hasselmann, 1971; McWilliams et al., 2004). 2758 Additional interactions take place through wave mass transport, enhanced bottom friction, and 2759 turbulence enhanced mixing, besides the normal component of the wind stress.

2761 Although circulation models often consider only the direct momentum transfer from wind to 2762 currents via surface wind stress, waves have their role as well in the process, with important 2763 implications, in particular for the mixing in the upper layer. The transfer of energy and momentum 2764 from the wind to the ocean via ocean surface waves influences the mixing dynamics through several 2765 ways (Ardhuin and Jenkins, 2005). The most intuitive one is wave breaking (Kantha and Clayson, 2766 2004), that inputs turbulent kinetic energy within a depth comparable with the order of magnitude 2767 of the wave height. The second way is via Langmuir cell generation or circulation effects, resulting 2768 from the interaction of turbulent vorticity with wave-induced Stokes drift, and that propagates in the 2769 whole mixed layer (McWilliams et al., 1997; Babanin et al., 2009). Last, the Coriolis-Stokes forcing 2770 needs to be accounted for, resulting from the interaction of large-scale planet vorticity with Stokes 2771 drift associated with ocean surface waves (Polton et al.,, 2005).

3.1.1 - A short review of coastal circulation modeling

where we outline the general accuracy of modeling coastal currents

Circulation models take many numerical forms, but produce fields of water levels and currents in either 2D horizontal (depth integrated) or 3D. Computational model grids may be structured or unstructured and the solution techniques may be explicit or implicit. Circulation models typically have time steps of seconds or smaller for accuracy and stability. Their inputs include bathymetry/topography, bottom friction coefficients, tidal forcing, atmospheric forcing (wind, pressure, air temperature, relative humidity, and more generally heat fluxes), Coriolis, river discharges, and short wave stresses. Turbulent shear stresses are expressed in the form of an eddy viscosity term. Circulation models generally give excellent results for idealized problems with analytical solutions (within a few percent) (e.g., Gerritsen et al., 2007). In coastal applications, however, simple approaches using constant vertical mixing are now generally substituted with more sophisticated approaches, e.g., two-equation turbulence closure models that account for wave breaking and Langmuir mixing induced processes (Sullivan and McWilliams, 2010; Kantha and 2788 Clayson, 2004). Inclusion of these processes are critical to reproduce the distribution of momentum fluxes within the mixed layer. 
2791 Solutions to field applications are more challenging. Circulation models rely on accurate 2792 bathymetric specification which can be difficult to obtain for complex coastal sites (e.g., tidal inlets, 2793 shoal complexes, tidal flats, and wetlands). The bathymetry is also often assumed to be stationary, 2794 even though it may change significantly on the time scale of a storm (surge) or of a tidal cycle. 2795 Narrow channels and steep bathymetry require locally a high grid resolution, which results in 2796 reduced time steps and longer computational times. To improve model stability, bathymetry 2797 features may be smoothed, reducing local accuracy. Accurate wind and pressure fields for complex 2798 storms, such as tropical cyclones, are also a challenge for accurate circulation modeling, especially 2799 in coastal areas. Circulation models focused on a certain area are very sensitive to the boundary 2800 conditions set in the model, and inaccuracies in radiating long waves at the boundaries can result in 2801 improperly reflected long waves trapped in the domain. Another frequent issue in circulation models is flooding and drying of land during tide cycles or surge events. Flooding and drying algorithms are ad hoc and can cause model instabilities and leave thin pockets of water on dry land during recession (Warner et al., 2010; Lesser et al., 2004) describe procedures to account for morphological updating in coupled flow-wave model systems. Some recent efforts have included the moveable-bed feature (Warner et al., 2008, 2010).

Despite these challenges, modern circulation models generally provide accurate representation of water levels. Modeling tides at 101 tidal stations on the US East and Gulf coasts and the Caribbean, the ADCIRC model (Luettich and Westerink, 2004) reproduced tidal constituent amplitudes within 6-13\% (Mukai et al., 2002), with the lower range of error in the dominant components. Errors in tidal constituent phases were 7-13 deg. Approximately half these errors can be attributed to measurements errors. Dietrich et al. (2012) modelled four recent hurricanes in the Gulf of Mexico 2814 (Katrina, Rita, Gustav and Ike) with ADCIRC tightly coupled to the spectral wave model SWAN 2815 (Booij et al., 1999), where tight coupling refers to the passing of water levels and depth-averaged currents directly in memory from ADCIRC to SWAN and radiation stress from SWAN to ADCIRC as the models run on the same grid (Dietrich et al., 2011, 2012). The modeled surge was evaluated with an unprecedented data set of measured water levels and high water marks (approximately 1,500 data sets over the four storms). The mean error for all four storms ranged from $-0.07 \mathrm{~m}$ to $0.15 \mathrm{~m}$, which is remarkable given the complexity of the modeling domains. Scatter Indices ranged from 0.16 to 0.28 .

2823 Water levels are a driver, but can also be considered an integrated product, of circulation. The key 2824 point is that limited differences in level between two locations can lead to substantial currents. This 
2825 implies that modeling of currents within circulation models is generally more challenging, and 2826 errors vary significantly with the location. Blain et al. (2010) validated ADCIRC for tidal currents 2827 at eight gauge locations in Delaware Bay (USA). Relative mean absolute errors ranged from 1 to $282835 \%$ for $2 \mathrm{D}$ and $3 \mathrm{D}$ simulations. The 3D model exhibited improvements in the estuarine region 2829 where there is complex stratification and mixing due to tides and river fluxes. Sutherland et al. 2830 (2004) evaluated two circulation models (DELFT3D and PISCES) at the mouth of the Teign estuary 2831 (UK). Waves were included in the simulations, but only water levels were fed back to the waves, 2832 not currents. Measured currents during the experiment ranged within $+/-0.5 \mathrm{~ms}^{-1}$. Relative mean 2833 absolute errors averaged over eight current meter measurements were approximately $70 \%$. 2834 However, current measurements are prone to errors (more than a tide gauge). Dealing with 2835 relatively limited currents, removing the estimated measurement error of $0.05 \mathrm{~ms}^{-1}$ reduced errors to $28363 \%$, a clear proof of the need to take instrumental errors into account (see Section 2.5 for the similar 2837 problem with waves). Hsu et al. (2008) evaluated DELFT3D for wave-driven longshore currents 2838 with data from two US beaches (Duck, North Carolina, and Santa Barbara, California). Normalized 2839 root-mean-square errors ranged from 21 to $30 \%$.

28413.1 .2 - The interaction with waves

2842 where we describe how currents affect waves

2844 Coastal currents and water levels impact oceanic waves by changing the wave length, speed, height 2845 and direction. The shape of the waves can also change making them nonlinear. Wave-current 2846 interaction is a "problem of wave propagation in an inhomogeneous, nonisotropic, dispersive, 2847 dissipative, and moving medium, which also interacts with the wave" (Jonsson, 1990). Wave2848 current interactions are calculated on the basis of conservation of wave action. Phase-averaged 2849 spectral wave models calculate wave-current interaction based on linear wave theory with currents 2850 that are assumed homogeneous over depth, although solutions of the dispersion equation for weakly 2851 sheared currents are available (e.g., Kirby and Chen, 1989). Depth-integrated currents are generally 2852 applied in modeling, but surface or wave orbital-weighted currents may also be applied in stratified 2853 environments (e.g., Elias et al., 2012).

2855 Waves entering a following current lengthen and reduce in height. Waves entering an opposing current shorten and steepen and may break. Tolman (1991) investigated the effect of spatial and temporal variations of currents in the Southern North Sea on wind generated waves, showing that 2858 these effects can significantly alter both the significant wave height and period measures. If 
opposing currents are of sufficient strength, they may completely block the waves (Lai et al., 1989).

2860 In tidal inlets with strong currents, wave heights may double or triple on ebb currents, and the 2861 increased height and steepness, with the consequent frequent breaking, can be a hazard to 2862 navigation. Wave-current interaction also impacts refraction and diffraction. In addition to 2863 constricted tidal inlets, strong coastal currents such as the Agulhas current off the east coast of 2864 South Africa, the Kuroshiro off east coasts of Taiwan and Japan, and the Gulf Stream off the 2865 southeast coast of the US interact strongly with waves. Examples of such interactions are nicely 2866 illustrated in Holthuijsen and Tolman (1991).

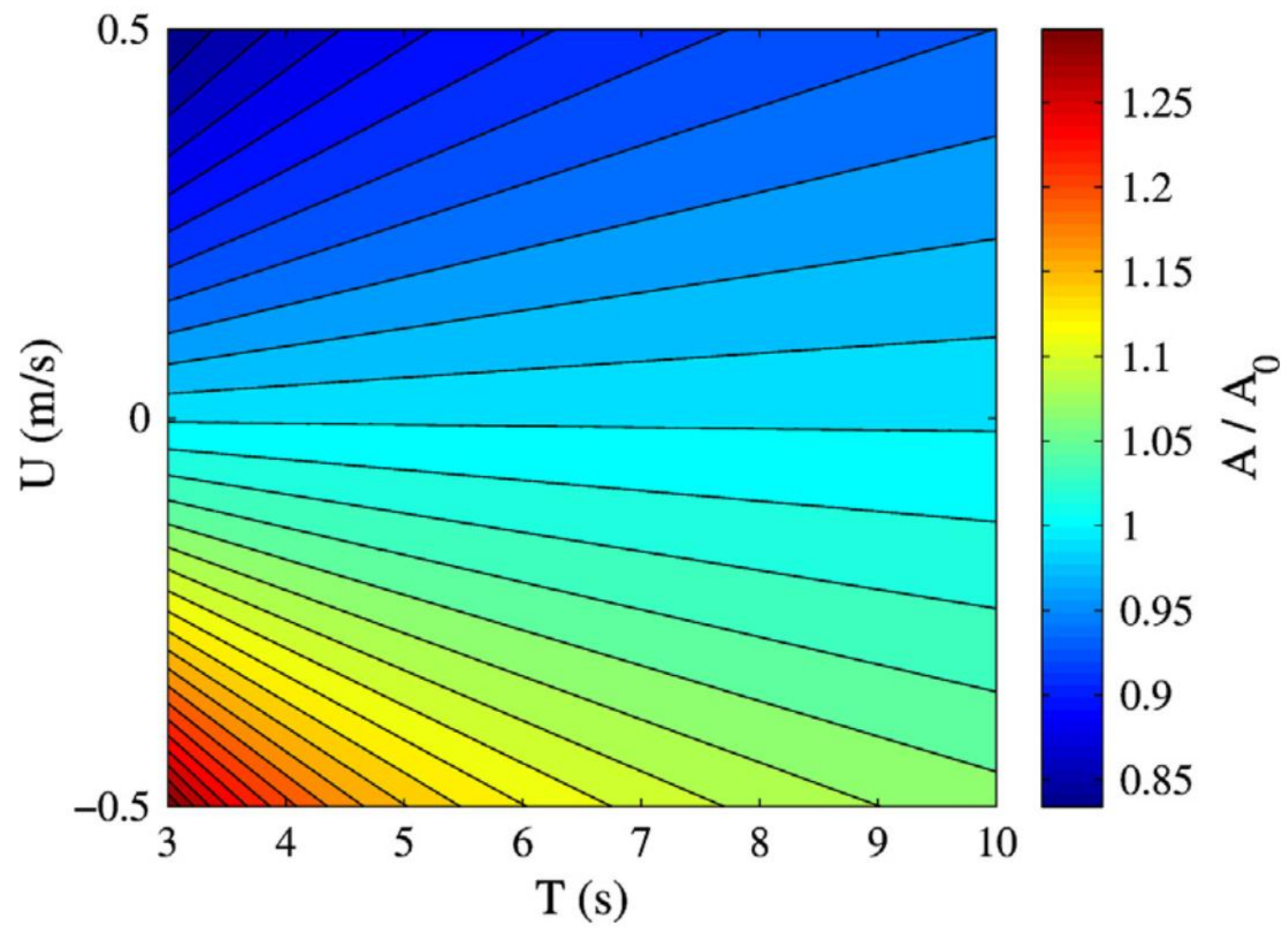

Figure 3.1 - How a following or opposing current affects the amplitude of a monochromatic wave, as a function of current speed and wave period.

The change of wave amplitude when entering a current is well summarized in Figure 3.1, providing the result for a range of current velocity $\left( \pm 0.5 \mathrm{~ms}^{-1}\right)$ and wave period (3-10s). Of course we are implicitly assuming that waves and current move in the same direction. If waves and current are at an angle, only the current component in the wave direction needs to be considered. However, the transversal current too has implications, in particular on the wave direction. The smaller the group speed, the stronger the effect will be. 

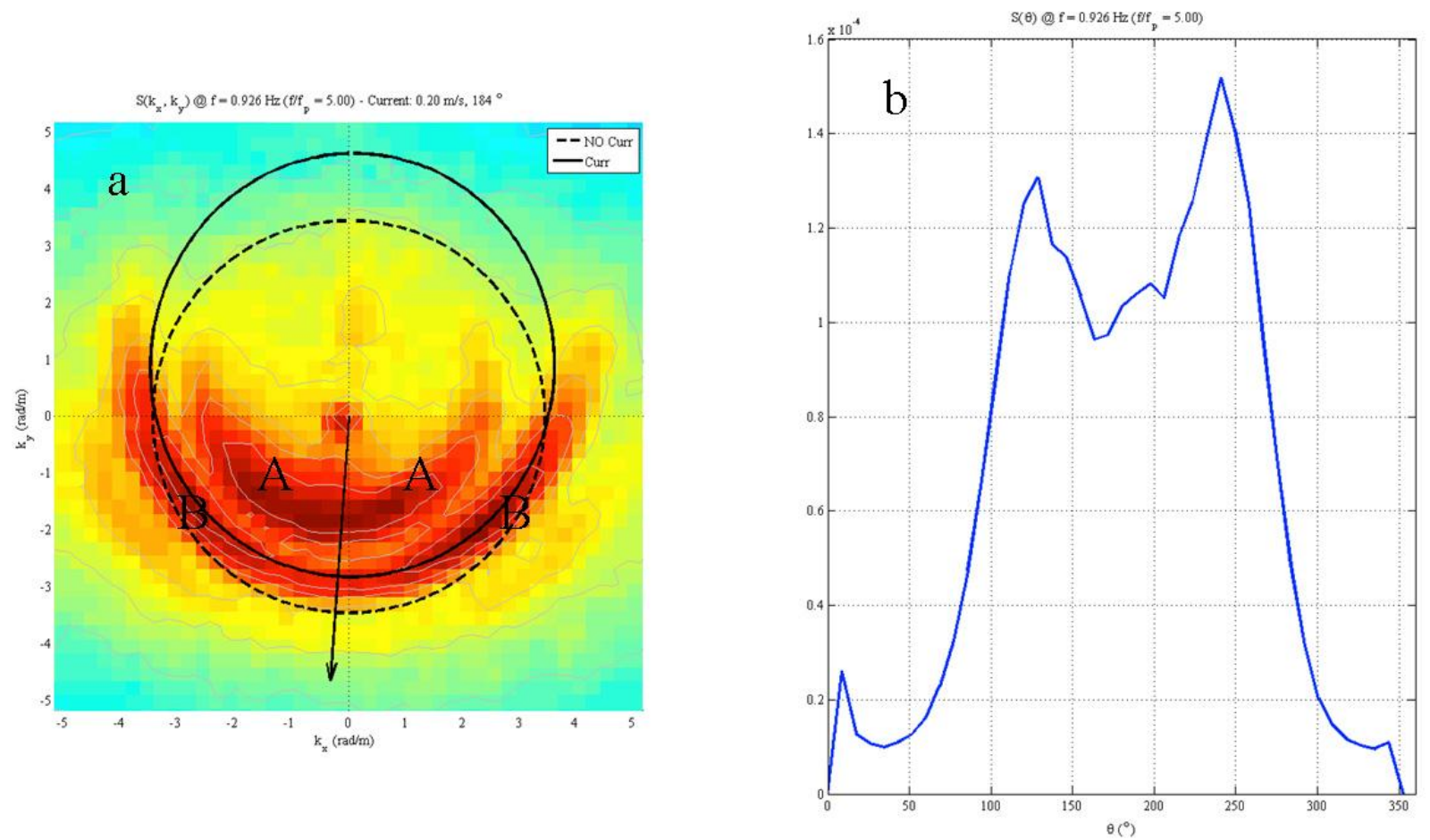

Figure 3.2 - a) Section, at measured frequency $0.926 \mathrm{~Hz}$, of a $\left(\mathrm{k}_{\mathrm{x}}, \mathrm{k}_{\mathrm{y}}, \mathrm{f}\right) 3 \mathrm{D}$ spectrum derived from stereo measurements of the sea surface. Dash circle: energy distribution according to the linear dispersion relationship. Continuous circle: actual energy distribution with respect to current. b) Directional distribution of energy along the continuous circle. Note the bimodality of the distribution associated to the non-linear interactions (see text for the full details).

A more interesting example, because it is actually measured, is shown in Figure 3.2. One of the several wave measuring systems on the oceanographic tower seen in Figure 2.3, panel d, is a stereo wave imaging system capable of providing high-resolution '3D+time' history of a large span of the sea surface upwind from the tower in a case of bora event (from North-East). Similarly to a case described in Section 3.1.4, bora waves are generally associated with a relevant current in the same direction. The stereo system provides high-resolution spectra both in frequency and direction (see 2892 Benetazzo et al., 2012, 2015). Figure 3.2 is quite rich in information. In the left panel, we plot the $\left(\mathrm{k}_{\mathrm{x}}, \mathrm{k}_{\mathrm{y}}\right)$ distribution of energy at apparent frequency $0.926 \mathrm{~Hz}$. What is shown is a section, at the indicated frequency, of the 3D distribution of energy $\left(\mathrm{k}_{\mathrm{x}}, \mathrm{k}_{\mathrm{y}}, \mathrm{f}\right)$ (see Holthuijsen, 2007, p.51, 3.5 .38 for a description of this approach). The dash circle shows the expected energy distribution according to the linear dispersion relationship. However, current was present with average speed $0.20 \mathrm{~ms}^{-1}$, in the indicated direction. This implies that the actual waves moving in the same direction were longer, hence at a lower frequency with respect to the current. Conversely, in the opposite direction, upward in the panel, the actual frequency was higher (shorter waves), but 
2900 delayed by current with respect to the tower, hence again at the apparent $0.926 \mathrm{~Hz}$. The full circle 2901 shows the actual wave number distribution of the waves, in all the directions, seen as $\mathrm{f}=0.926 \mathrm{~Hz}$ by 2902 the fixed stereo system. The comparison between the two circles provides a clear idea of the 2903 current-induced Doppler effect on waves. However, there is much more in panel a. The distribution 2904 of energy in the main wave direction (practically coincident with the current) is evident, but evident 2905 are also the hyperharmonics, A-A as the arc of energy internal to the full circle. Not only that, along 2906 the full circle, at about $\pm 45^{\circ}$ with respect to the main direction, are two peaks of energy $\mathrm{B}$, better 2907 seen in the right panel providing the energy density along the circle, i.e., at $0.926 \mathrm{~Hz}$ (with respect 2908 to the current). The obvious remarkable feature are the two just mentioned peaks that represent the 2909 effect of non-linear interactions in the frequency range just above the 1D peak.

2911 The steepening of waves in sufficiently intense and opposing currents has the double implication of 2912 increasing their non-linearity and, as cited, leading possibly to breaking. Chawla and Kirby (2002) 2913 found that wave breaking criteria based on steepness work well for this situation, but require 2914 coefficient modifications compared to the standard breaking case. Also, they found that the 2915 significant steepening of the waves requires that nonlinear terms in the dispersion relation 2916 become important and must be included. Ardhuin et al. (2012) evaluated dissipation rates 2917 proportional to wave steepness to the fourth power, but found none of the parameterization to be 2918 fully satisfactory. This points to the need for more measurements and improvements to 2919 parameterizations.

2921 As waves on an opposing current become steeper, hence possibly strongly nonlinear and near 2922 blocking, sideband instabilities develop that play a crucial role in the dynamics close to the 2923 blocking region (Lai et al., 1989). In this situation energy shifts from the peak frequency to both 2924 higher and lower sidebands. If blocking occurs at the peak frequency and above, energy in the 2925 lower sideband may still penetrate the current. Spectral wave models do not account for this 2926 nonlinear frequency downshifting due to wave modulation, and thus would predict total wave 2927 blocking, when downshifted energy exists. Like Chawla and Kirby (2002), Dodet et al. (2013) 2928 suggest that the application of a higher-order dispersion relationship would improve modeled wave 2929 heights on strong currents.

2930 Water level variation from tides or surge impact waves in an expected way. Increases in water 2931 levels allow larger waves to penetrate deeper into estuaries at high tide or even larger distances 2932 inland with large storm surge. Indeed waves riding on storm surge do much of the destruction of 2933 infrastructure during hurricanes (Kennedy et al., 2011). One of the unresolved difficulties of 
2934 modeling this "overland" wave propagation is a good parameterization of wave dissipation for 2935 interaction with buildings, urban infrastructure, and terrestrial vegetation. Waves propagating over 2936 shallow shoals are modulated based on the local wave height to water depth ratio. In this respect, 2937 see the recent thesis and related publications by Salmon (2016) and Salmon et al. (2015). For 2938 example, Smith et al. (2000) applied STWAVE and ADCIRC to model waves, currents, and water 2939 levels at the entrance to Willapa Bay (see Section 3.1.4 for more details). The results demonstrated 2940 that the dominant transformation process for waves in the bay was wave breaking over the Willapa 2941 bar, and the energy dissipation was controlled by the tide elevation.

2943 From the above it is obvious that waves are particularly sensitive to strong current gradients, and 2944 indeed most of the impact is found in areas downwave of the gradient zones. For larger scale 2945 variations the impact are less extreme, and Ardhuin et al. (2012) found that for larger scale current 2946 variations, the observed modifications of the sea state are mostly explained by refraction of waves 2947 over currents and relative wind effects (wind speed relevant for wave generation is the speed in the 2948 frame of reference moving with the near-surface current). Introducing currents in wave models can 2949 reduce the errors in wave heights by more than 30 percent in some macrotidal environments, such 2950 as the Brittany coast in France.

2952 In 3.1.1 we mentioned the possibility, and eventually the need, to model currents with a 3D 2953 approach. Sheared currents are a frequent reality, especially in shallow areas if considering the 2954 implications for their interaction with waves. Indeed in these conditions an improvement of mean 2955 period is reached by considering the 3D Doppler shift as formulated by Kirby and Chen (1989). 2956 This process is important in sheared flows where the use of a depth-averaged current induces errors 2957 on the wave-current interaction estimation for the different wave components. The influence of 2958 bathymetry is also seen to be stronger on the wave field when considering the 3D Doppler shift. 2959

2960 Expanding our look to wave measurements, in addition to modeling waves, wave-current 2961 interaction needs to be considered in the analysis of wave information derived from subsurface 2962 pressure and currents or radar measurements. Waves ride a current according to their dispersion 2963 relationship. However, seen from the static point of view of an instrument at a fix position, their 2964 phase speed is apparently different, larger or smaller depending if waves follow or move against the 2965 current. A one point fixed gauge measures the period of the waves, therefore current is necessary 2966 information to derive the correct wave period, hence spectrum. The higher the frequency, the more 
2967 crucial this is, not only because of the stronger relative frequency shift due to current, but also for 2968 the higher amplification of the signal once transformed to the surface.

2970 3.1.3 - Two-way coupling

2971 where we point out the reciprocal and conditioning actions between waves and currents

2972 Granted that waves may generate currents and the latter affect the former in various ways, it is 2973 obvious that the actual truth hides a continuous interplay with reciprocal interactions. These can be 2974 more manifest in certain areas and require specific numerical approaches.

2976 As waves break under the action of wind or in near coastal regions, momentum is transferred from 2977 the wave field to the mean circulation. Near coast longshore currents and cross-shore water level 2978 variations are generated. In bathymetrically complex nearshore regions, strong circulations 2979 including rip currents may be generated. These current and water level patterns can feedback into 2980 the modification of the wave field through wave-current and wave-water level interaction. Kumar et 2981 al. (2012) and Uchiyama et al. (2010) compare radiation stress and vortex flux formulations for 2982 wave effects on 3D currents (including 3D wave stresses). Non-conservative wave effects on 2983 currents include wave-enhanced vertical mixing due to wave breaking and surface rollers, bottom 2984 streaming stress due to near-bed wave drag, and wave-enhanced bed shear stress (e.g., Soulsby, 2985 1995). Formulations for wave breaking enhanced mixing are generally empirical.

2987 On the sea bottom, the wave-enhanced turbulence in the Bottom Boundary Layer (BBL) modifies 2988 the boundary conditions for momentum in the Reynolds-averaged Navier-Stokes equations and 2989 determines the rate of sediment bedload and resuspension. Locally, the non-linearities involved in 2990 combined wave and current dynamics may produce a relevant increase in bottom shear stress and 2991 thus in sediment mobilization (Soulsby, 1995). State-of-the-art BBL sub-models include current 2992 velocities at a reference elevation close to the bed, bottom wave period, bottom wave orbital 2993 velocity, and wave direction, in order to account for the combined effects of waves and currents 2994 (Soulsby, 1995). WCI has a twofold influence on sediment transport: on one hand, the presence of a 2995 flow field modifies the geometric features of waves, while on the other hand the wave contribution 2996 to the water column momentum can generate relevant modifications in the local circulation features. 2997 This implies that both bottom stress and advective flow fields may be affected by WCI, in turn 2998 affecting both sediment suspension and transport (Sclavo et al., 2013). 
3000 The incorporation of wave effects (subgrid scale) in the form of wave-averaged bottom stresses and 3001 wave-averaged sediment transport rates remains a challenge. The wave boundary layer thickness is 3002 small compared to the layer thicknesses usually applied over the vertical in practical applications. 3003 This will remain a challenge for modeling systems that integrate hydrodynamics, waves, and 3004 sediment transport to predict morphological changes.

From the numerical point of view, wave-circulation coupling has been approached in a number of previous studies that consider two-way coupling between currents and waves (see, among others, Bolaños et al., 2011; Malhadas et al., 2010; Dietrich et al., 2011; Benetazzo et al., 2013; Benetazzo et al., 2014). The coupling may be achieved via direct connections within the codes or using couplers that provide efficient interpolation methods and message passing routines, but make communication of parameters less transparent and more difficult to debug. For this purpose, appropriate coupling software must be adopted (see e.g., Larson et al., 2004; Jacob et al., 2005; 3013 Warner et al., 2008) which exchanges matrices between the different modules and interpolates 3014 between different coordinate systems.

Additionally, we should recall that parameterizations for most state-of-art models have been calibrated and consolidated to mimic observed dynamics in the absence of an explicit coupling within a complete coupled wave-current-atmosphere system. Besides improvement in the physical insight, the benefits of a shift towards a coupled approach in terms of model performances are therefore expected to appear progressively, as further fine-tuning is carried out based on the new modeling framework. For this reason, it is not straightforward to generalize evaluations of coupled versus uncoupled model skill, and a systematic comparison against field and laboratory observations is still required.

\subsection{4 - Practical examples}

where we describe cases where waves and currents interact at various levels of complexity

Having provided the background for the possible interactions between waves and currents, it is useful and mandatory to provide examples where this happens and, where available, to quantify the implications. We consider examples at different scales, from the relatively small one of the exit of a river to the still enclosed, but wider span of the Gulf of Mexico. 
3034 The Dee estuary, close to Liverpool, U.K., provides a good example of the impact of currents on 3035 waves. The river flow is not big in itself, but the remarkable range of the local tide, up to several 3036 meters in spring conditions, leads to tremendous currents. Exposed to the active and potentially 3037 violent Irish Sea, full consideration of the effects of currents and local changing depth on the wave 3038 characteristics is a mandatory condition. Brown et al. (2013) have implemented a tide-set-up-surge3039 current-wave coupled system in the whole Irish Sea with a nested high-resolution version in 3040 Liverpool Bay. The much stronger gradients in the bay require a much reduced integration time step 3041 (30 s instead of $200 \mathrm{~s}$ ). To illustrate how careful we need to be in coupling, we quote the difficulty 3042 Brown et al. (2013) report with radiation stress. Initially a 3D method (Mellor 2005) was coded 3043 (Bolanos et al. 2011). However, in shallow water this approach (Mellor 2011) led occasionally to 3044 spurious accelerations, in particular just outside the surf zone. In turn this implied unrealistic coastal 3045 circulation. In the end, a 2D radiation stress approach was preferred (Mastenbroek et al. 1993), 3046 leading to quite realistic results for wave set-up and induced circulation.

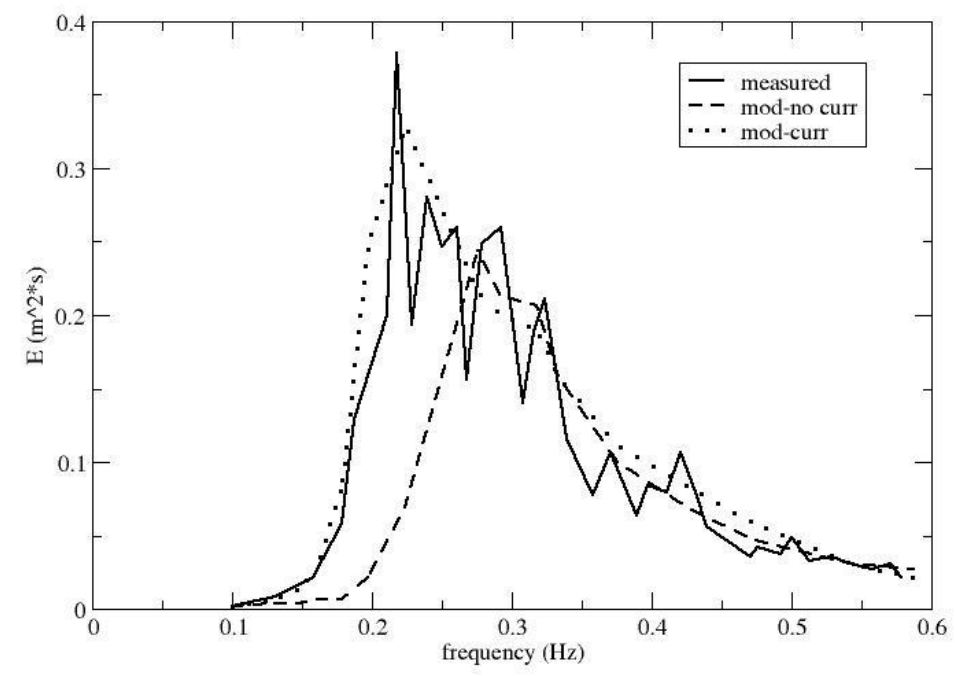

3048 Figure 3.3 - Wave spectrum modification by an opposing current, as seen by a stationary observer.

- Frisian Islands

3051 The combination of a former ice age, lower and then rising sea level, tide, and wind led in the 3052 millennia to the formation of the Frisian Islands, a $500 \mathrm{~km}$ line of dunes off the coast, cut through 3053 by the sea at several locations, and extending from Netherlands to Germany and Denmark. This 3054 leads to remarkable currents in the various inlets between successive islands. These currents interact 3055 with the frequent, potentially violent, storms of the North Sea. Groeneweg et al. (2008) used the 3056 SWAN model to estimate the wave conditions in the inlets. As expected, they found that taking 
local currents into account is a necessary condition to get reasonable wave results. The local environment is particularly difficult because of the local strong spatial gradients for both waves and currents. Figure 3.3 provides a nice example of the need of coupling for meaningful results. 3060 Compared to the measured spectrum $(\mathrm{Hs} \approx 0.7 \mathrm{~m})$, we see the model spectra without and with consideration of current. When compared to measurements, the better fit with current is evident.

- Southern North Sea
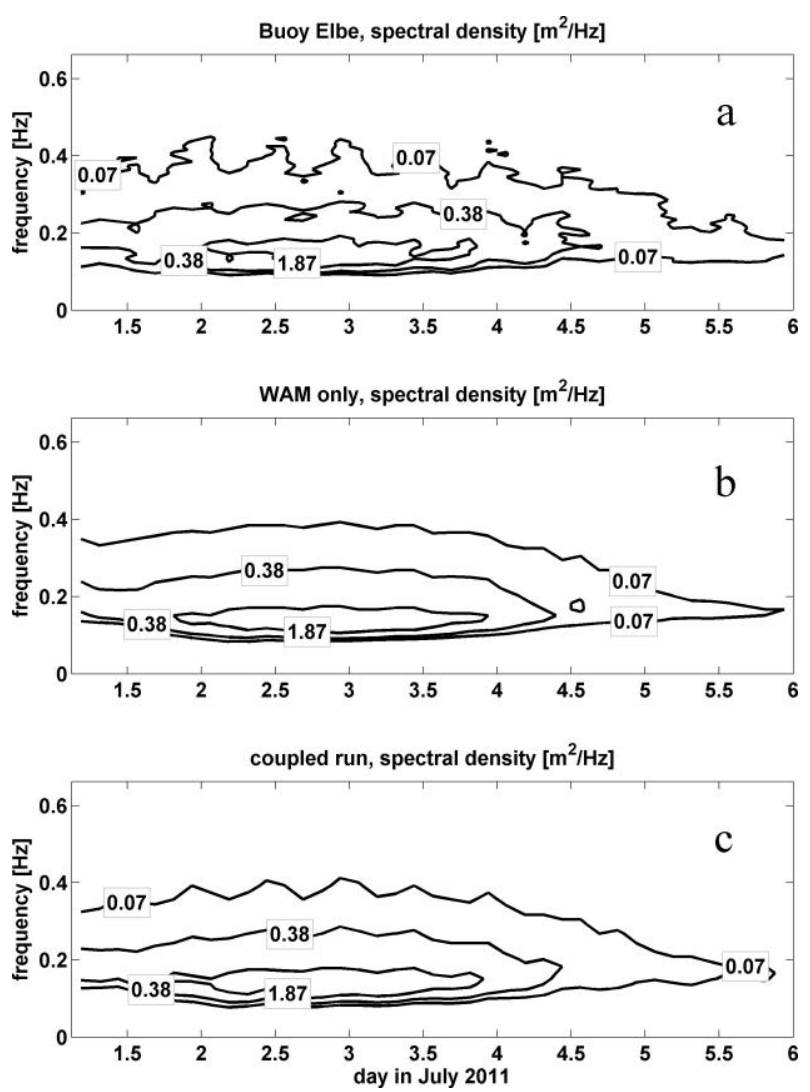

3066 Figure 3.4 - German Bight, Southern North Sea. Sequence of spectra as measured and estimated at the local Elbe buoy (f vertical, time horizontal). Isolines of 1D energy density are shown. a) Note the modulation by tidal current in the measured spectra. b) and c) Uncoupled and coupled run respectively. The only slight modulations in b) are due to a modulation of the driving wind.

3071 The southeastern North Sea (the German Bight) is dominated by strong tidal currents exceeding in 3072 some areas $1 \mathrm{~ms}^{-1}$. Therefore the feedback between currents and waves plays an important role in 3073 this area, and wind wave-circulation coupling needs to be accounted for, especially during extreme 3074 events. Figure 3.4, panel a, shows how the locally measured spectra at the Elbe buoy station varied 3075 during a mild storm (maximum $\mathrm{H}_{\mathrm{s}} 2.5 \mathrm{~m}$ ) during the first five days of July 2011. There is an 3076 obvious modulation of the spectra at half a day interval, i.e. with the semi-diurnal tide. Note, as 
expected, how the modulation is stronger in the high frequency range (up in the panel) due to their 3078 reduced group speed with respect to the current. The uncoupled wave model results in panel $b$ show 3079 only a very slight modulation, expected to depend on the variable wind forcing, because wind too 3080 varies with tide and temperature. It is only in the coupled model (panel c) that we recognize the full 3081 12-hour cycle in the modulation of the spectra with a good fit with the results in panel a. The tidal 3082 currents are mainly affecting the tail of the spectra, whereas the energy around the peak is not much 3083 different in all three panels. The statistical analysis of the results (see Staneva et al., 2016) confirms 3084 what is already evident from the figure.

- Willapa Bay

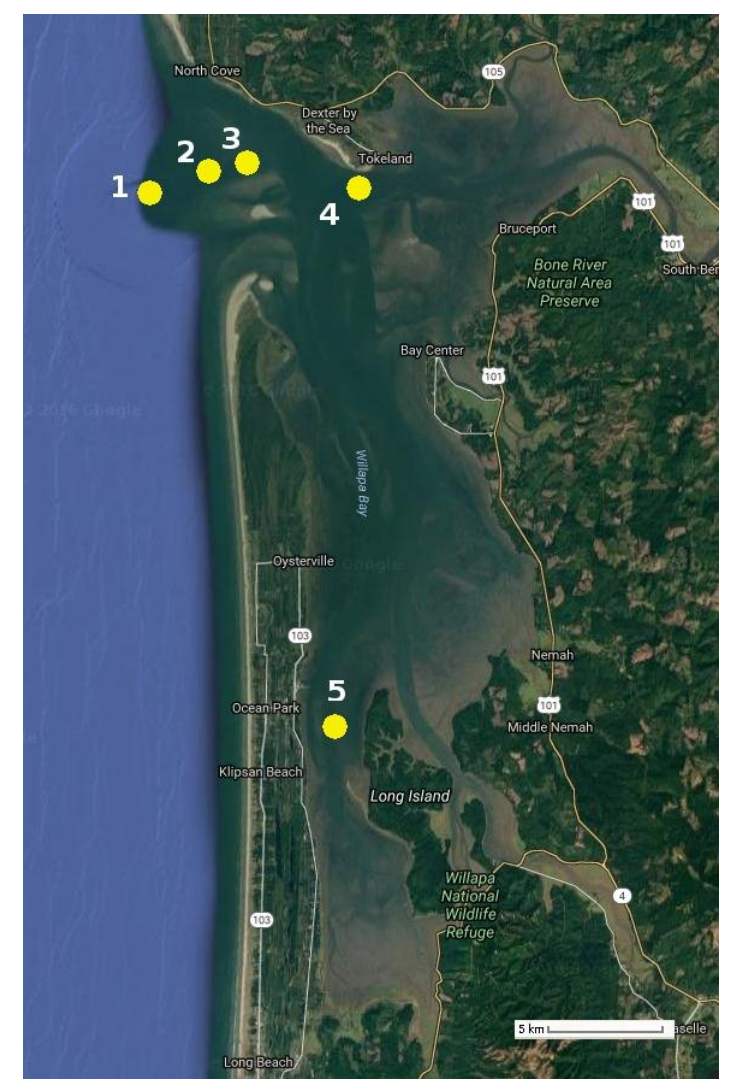

3088 Figure 3.5 - Willapa Bay (Washington State, USA). The dimensions (see scale) are about 40x10 $3089 \mathrm{~km}$. Numbers indicate the location of the measuring buoys.

3091 The entrance to Willapa Bay (Washington, USA, see Figure 3.5) includes a complex of shoals and a 3092 meandering channel. The mean tide range is $2.7 \mathrm{~m}$ with peak currents of over $2 \mathrm{~ms}^{-1}$. The average 3093 yearly wave height is $2 \mathrm{~m}$ with storm heights up to $9 \mathrm{~m}$. In the entrance channel, Smith et al. (2000) 3094 found that the effect of currents on waves was most significant in the outer entrance channel, where 3095 wave heights on the ebb increased up to 80 percent and on the flood decreased up to $20 \%$. A 3096 remarkable fact is that the modulation of the waves in the upper part of the bay (points 2, 3, 4), 
3097 affected by the conditions offshore (point 1), depend strongly on the tide via the shoal at the 3098 entrance and the consequent variable bottom induced wave breaking. Olabarrieta et al. (2011) found 3099 that also the locally generated waves in the bay (point 5) are strongly modulated by the transversally 3100 non-uniform tidal flow via the tunneling or detunneling effect. If the flow is stronger at the center of 3101 the local channel, opposite moving waves will tend to focus at the center of the channel. The 3102 opposite is true for following waves.

- North Adriatic Sea

3105 The North Adriatic Sea (see Figure 2.3, panel a for its location) has the double advantage of a) strong bursts of bora wind with consequent high locally generated waves and resulting currents, and b) the availability of a fully instrumented oceanographic tower (panel d) located $15 \mathrm{~km}$ off the coast of Venice. Benetazzo et al. (2013) implemented the first fully coupled wave-ocean 3D model (COAWST suite) in the Adriatic sea region, where the current passed from the ocean to the wave model was based on the Kirby and Chen (1989) formulation (see above), which computes a weighted depth-averaged velocity accounting for the vertical current structure and the dispersion relation of surface gravity waves. The importance of vertically averaging the currents over a depth controlled by the spectral wave numbers was found to be important in shallow waters, where almost

3114 the entire vertical current shear affects wave dynamics. With this approach Benetazzo et al. (2013) were able to show that the presence of current, up to $0.6 \mathrm{~ms}^{-1}$, led to $0.6 \mathrm{~m} \mathrm{H}_{\mathrm{s}}$ reduction with respect to the "only waves" case. At the same time it turned out that $10-15 \%$ of the current speed was due to the presence of waves. We will go into more details for this storm in Section 3.2, discussing the interaction with the atmosphere.

\section{- Hurricane Isabel}

3121 Warner et al. (2010) developed and applied the COAWST system to a hurricane scenario, forced 3122 with the atmosphere Weather Research and Forecasting model (WRF) (Skamarock et al., 2005). 3123 They showed that in certain conditions the significant wave height increases by as much as $20 \%$ 3124 when a wave system meets an opposing current. Similar results were obtained by Fan et al. (2009) 3125 who found a reduction in the wave energy when including an oceanic current following the waves. 3126 The authors also highlight that wave-current interaction improves hindcasts and forecasts of wave 3127 energy and, as a consequence, significant wave height. Figure 3.6, left panel, shows the Gulf Stream 3128 off Cape Hatteras interacting heavily with the waves produced by Hurricane Isabel (18 September 3129 2003, 12 UTC) whose eye at this time is in the lower central part of the panel. In the right panel we 3130 see the wave height differences due to the interaction with the current field. To the left of the eye, 
waves move against the current, hence the line of increased heights along the border of the continental shelf. On the contrary, just east of the cape, the maximum estimated wave heights at this stage of the hurricane, up to more than $18 \mathrm{~m} \mathrm{H}_{\mathrm{s}}$, are locally decreased by a strong component in 3134 the direction of the current.
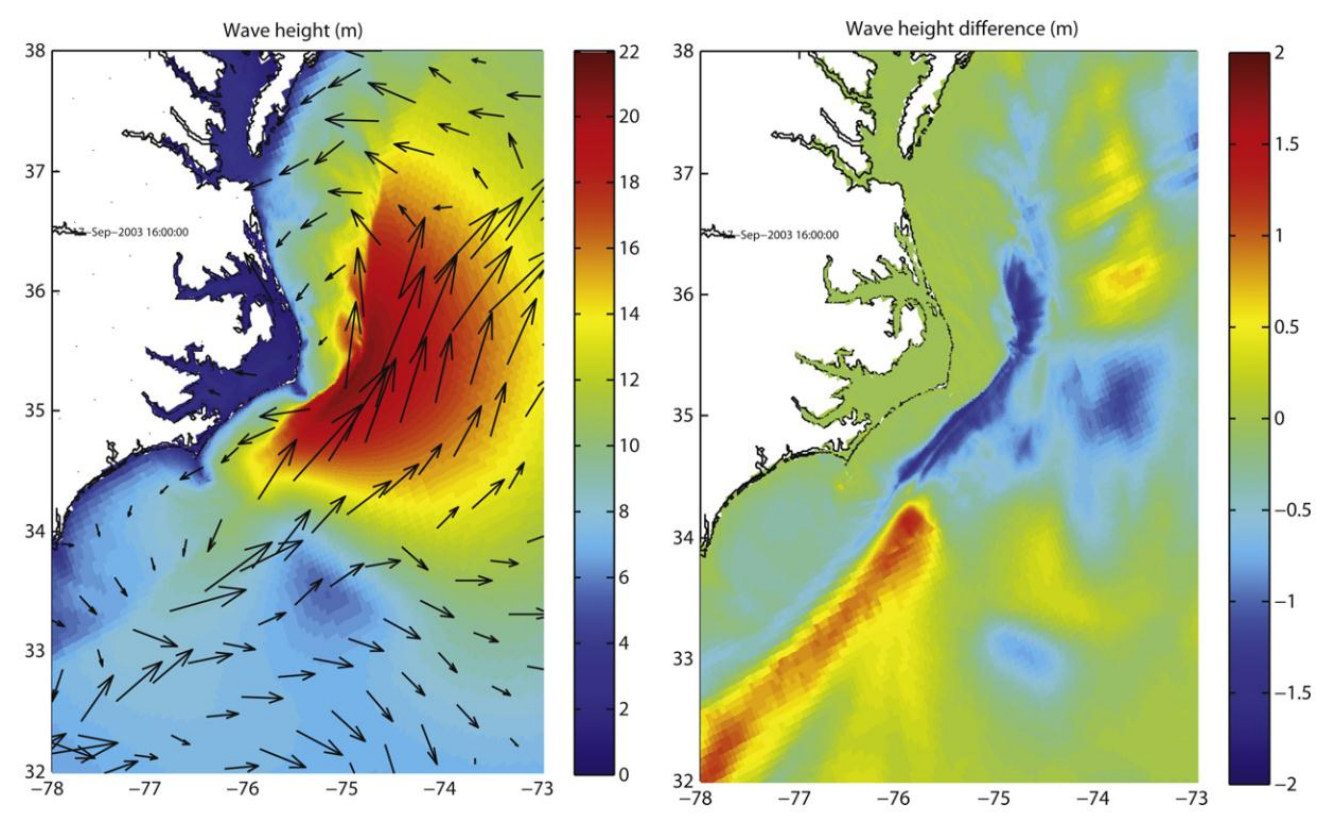

Figure 3.6 - Hurricane Isabel, 18 September 2003. a) Interaction between the hurricane generated waves (colors) and the Gulf Stream (arrows) around Cape Hatteras. At the time of the plot the eye was located in the lower central part of the figure. b) Wave height differences with-without wavecurrent interaction.

- Northern storm on the Gulf Stream

A similar case, but in less extreme conditions, is shown in Figure 3.7. The left panel shows large wave heights, 5-6 $\mathrm{m} \mathrm{H}_{\mathrm{s}}$, from a northerly storm along the coastline of Melbourne (Florida, USA), estimated without considering the interaction with the local current field. When the Gulf Stream (panel b) is taken into account, the overall $\mathrm{H}_{\mathrm{s}}$ field is substantially enhanced (panel c). Note the elongated shape of enhanced area, practically superimposed on the Gulf Stream. Note in particular in panel $\mathrm{c}$ the lateral convergence of wave energy towards the enhanced area following the wave

Moving closer to coast, we report now the results for two events of completely different magnitude,

3152 but both showing the reciprocal role of waves and water level in determining the conditions at the coast. 

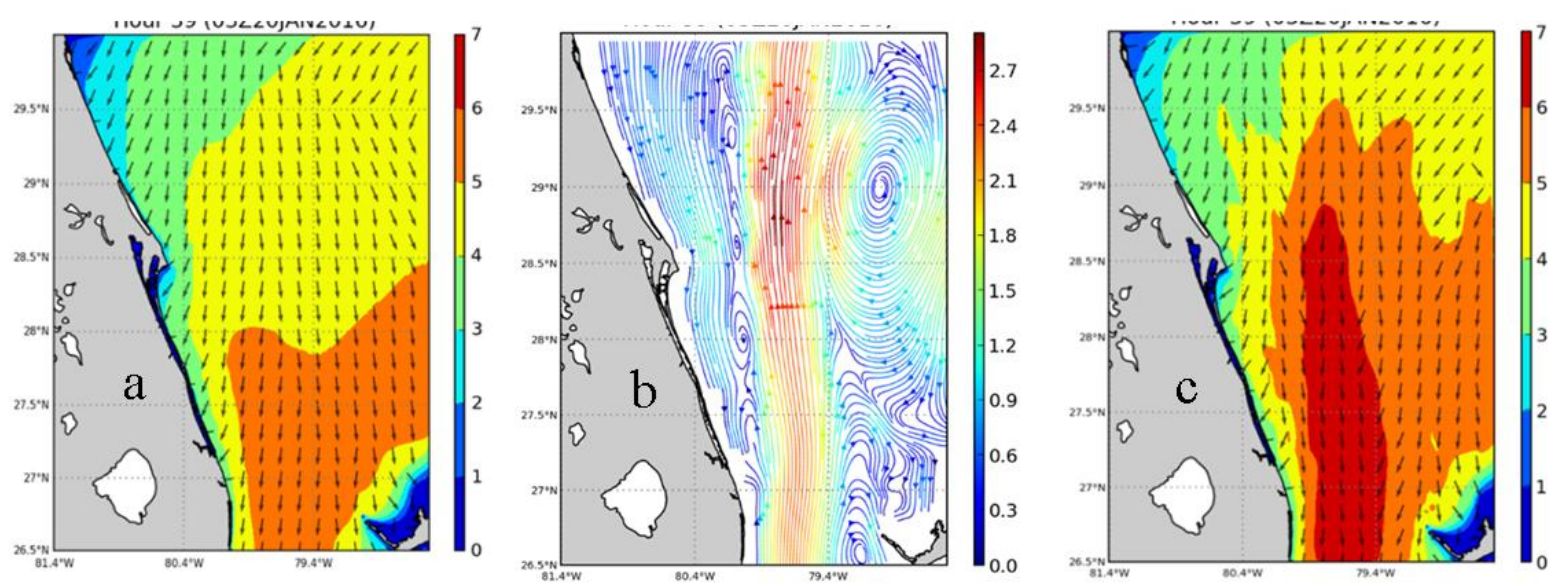

3157 Figure 3.7 - Interaction between the Gulf Stream and a northerly storm off Melbourne (Florida, 3158 USA). a) The uncoupled wave field, b) the Gulf Stream distribution, c) the coupled wave field. Note 3159 the much increased wave heights and the wave lateral convergence towards the Gulf Stream due to current induced refraction

- Hurricane Katrina

3163 Katrina, August 2005, was the costliest natural disaster, and one of the five deadliest hurricanes, in 3164 the USA documented history. Reaching peak strength in the Gulf of Mexico, Katrina landed just 3165 east of New Orleans. Much of the damage on the coast and the inland area (up to several 3166 kilometers) was due to the increased level of water and the consequent action of waves. Figure 3.8, 3167 three panels, show respectively: a) the maximum significant wave height reached in the area around 3168 the delta of the Mississippi river, b) the maximum wave set-up, c) the maximum water level 3169 elevation. Of course all these quantities are reciprocally related. Large wave heights arriving at, and 3170 breaking on the shallow areas of the delta leads to wave set-up in a positive loop involving both 3171 these quantities. All this is further enhanced by the overall storm surge (note the $8 \mathrm{~m}$ coastal surge 3172 about $70 \mathrm{~km}$ east of New Orleans). We will come back to this last point, more specifically panel c, 3173 in Section 3.2 dealing with the reciprocal interaction with the atmosphere. 

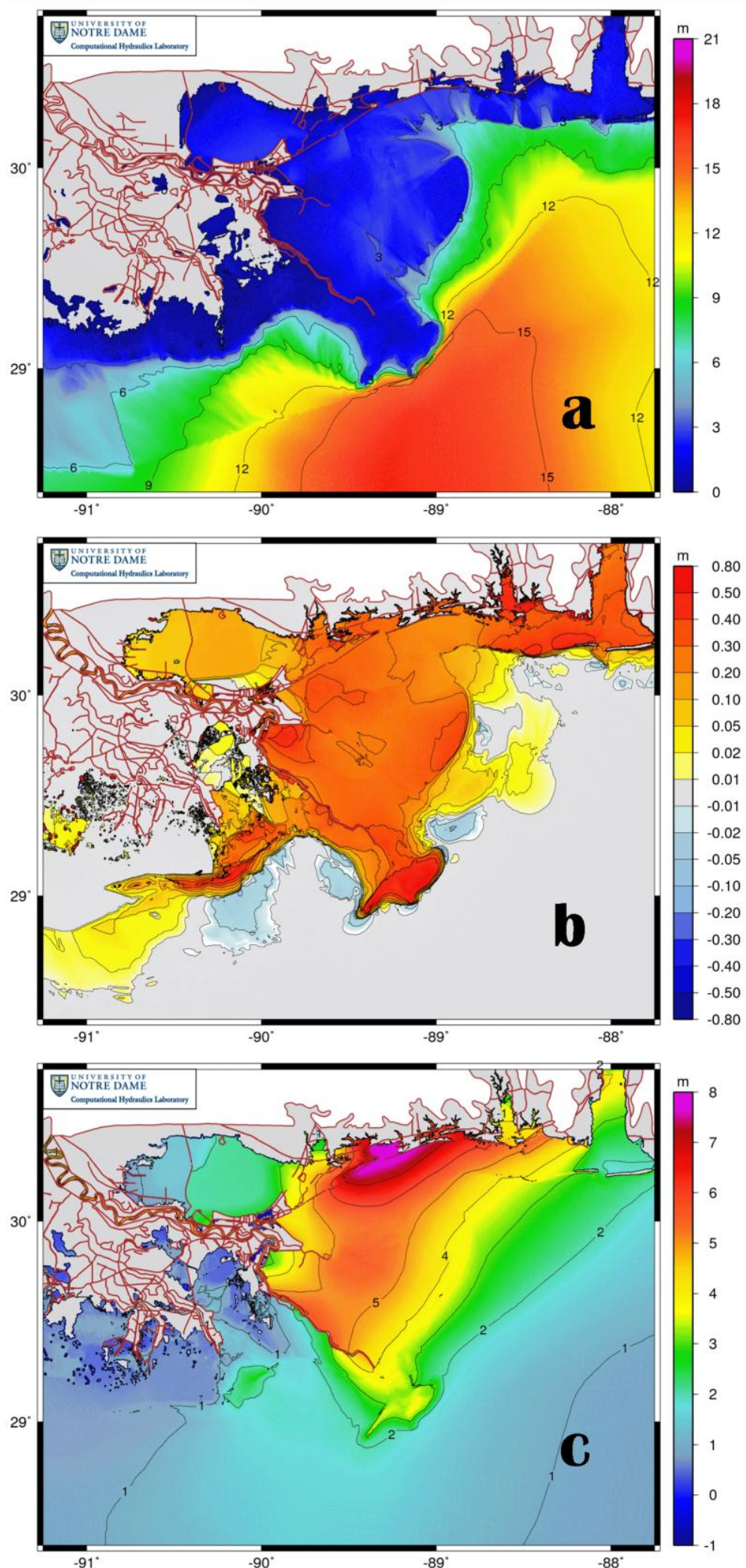

3176 Figure 3.8 - Hurricane Katrina, August 2005. Area around and to the East of the Mississippi delta.

3177 Distributions of maximum a) significant wave height, b) wave set-up, c) water level (courtesy of 3178 Casey Dietrich, North Carolina State University) 
- Wave set-up at Venice coast

3182 Much smaller wave heights and surge were involved in the storm that hit the Northern Adriatic Sea 3183 (Figure 2.3) on 22 December 1979. The flood ranked as the second most severe in Venice history, 3184 with $1.66 \mathrm{~m}$ above the nominal local sea level. The availability of tidal data at the oceanographic 3185 tower (panels c, d) and at the jetties at the entrance of the lagoon made it possible to measure the 3186 different sea levels. Figure 3.9 shows the $\mathrm{H}_{\mathrm{s}}$ values at the tower and how the difference varies through the storm. We point out that after the nominal 20 hours, the wind had changed direction so that it was not blowing any more towards the coast. Therefore after this time the difference was due only to wave set-up, duly decreasing in time with the wave height. Bertotti and Cavaleri (1985) estimated the set-up with a wave-sea level coupled model starting from a general hindcast of the storm and the conditions at the tower where, according to the hindcast, waves were already limited by depth-induced breaking, hence we were already in a set-up regime. The shown differences are not with respect to the coast, but to almost two kilometers offshore, at the end of the jetty at $6 \mathrm{~m}$ depth. The maximum coastal set-up was estimated at more than $0.5 \mathrm{~m}$ with respect to the tower.

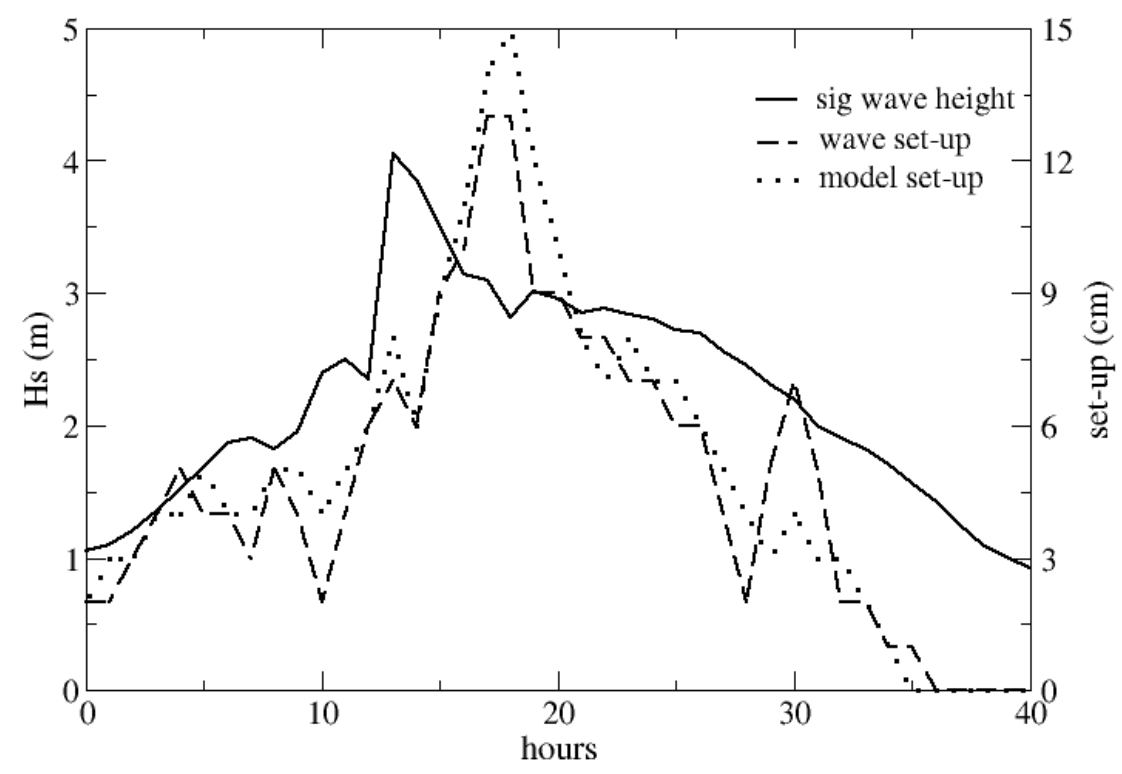

Figure 3.9 - Wave set-up on the Venice coast during the storm of 22 November 1979. The set-up is between a tide gauge $2 \mathrm{~km}$ offshore (at the end of a jetty, $6 \mathrm{~m} \mathrm{depth}$ ) and the one on the oceanographic tower $15 \mathrm{~km}$ offshore. See Figure 2.3 for details. The significant wave heights are referred at the tower position (16 $\mathrm{m}$ depth). 
- Catalan coast

3203 We end this section with a formally negative example that will open the way to the next section of interaction with the atmosphere.

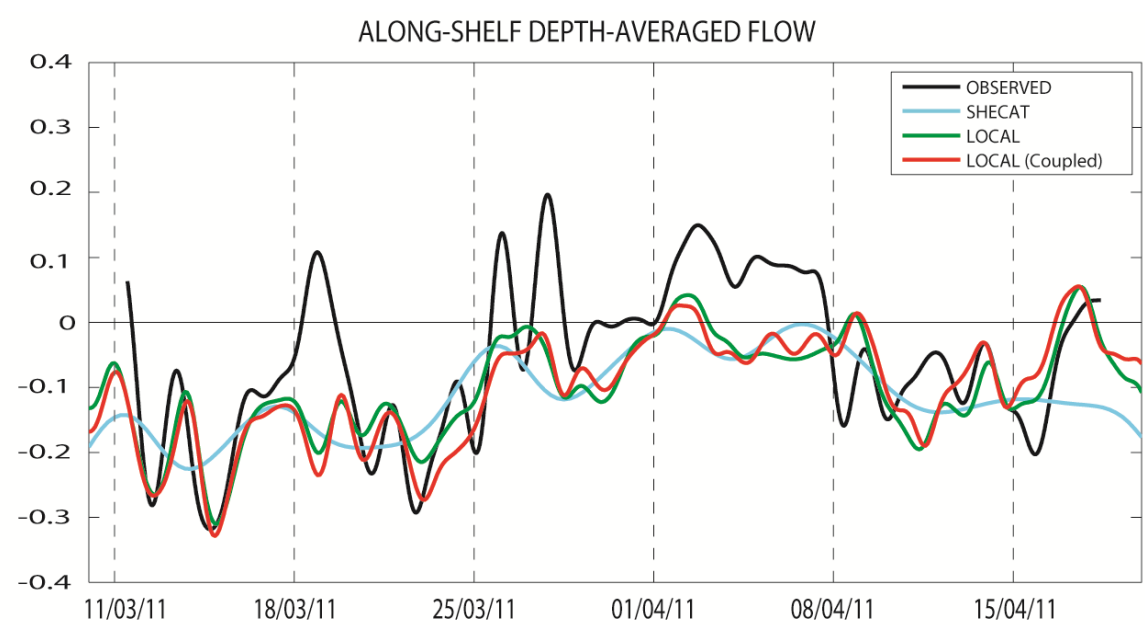

3207 Figure 3.10 - Currents measured and estimated just off the Catalan coast. The Shecat model has 1 $3208 \mathrm{~km}$ resolution. Local, uncoupled and coupled, $50 \mathrm{~m}$ resolution. Observed data from local currentmeters.

The Catalan coast is at the north-western end of the Mediterranean Sea (Figure 2.3, panel a). It is exposed to mainly easterly winds, but with often sustained wind storms from inland. Figure 3.10 shows the along-shelf, depth-averaged flow component just off the coast as measured by local current meters. The three color lines show the results of the local circulation modeling at $1 \mathrm{~km}$ (SHECAT) and (two-step nesting) 50-m resolution. Finally the corresponding results for the fully coupled (WRF, SWAN, ROMS) system are shown. In general the three simulations do not differ dramatically, although occasional larger differences associated with coupling appear. However, the main message comes from the comparison with the measured data. There are repeated, also extended, occasions when the measured data are completely different, well off the set of the modeled data. Sanchez-Arcilla et al. (2014) attribute this to transient forcings of the atmospheric driver. The lesson to be derived has been known for decades: we can have all the possible sophisticated approaches in our model, however, if the atmospheric input is not sufficiently correct, all the couplings we consider will not necessarily move the final result in the right direction. Still with much attention, this encourages us to move to full coupling with the atmosphere. 


\section{2 - The coupling of ocean waves with the oceanic and atmospheric boundary layers}

3229 where we involve the atmosphere in the interplay between ocean and waves

3231 Until recently, the oceanic wave field and the interior ocean were modeled as separate entities, each 3232 forced independently and inconsistently by atmospheric fluxes of momentum, heat and latent heat 3233 with no feedback to the atmospheric boundary layer. Climate models were naturally the first to delve 3234 into coupling of the ocean and the atmosphere, but also these models refrained from including the 3235 oceanic wave field. Except for the coupled atmosphere-wave forecasts issued by ECMWF since 1998 3236 (Janssen, 2004), no attempt was made at coupling operational atmosphere-wave forecasts, let alone 3237 fully coupled atmosphere-wave-ocean forecasts. Only very recently Breivik et al, (2015) acted in this direction.

Interestingly, the most active part of the sea is its surface, and ocean waves play a key role in modulating, in a direct or indirect way, all the exchanges at the surface. Because in turn some of the effects feed back to the wave field and the upper ocean,, all this is of interest also for our present 3243 purpose.

3244 In this section we deal with the full coupling of the ocean--wave-atmosphere system. This requires a clear view on the processes in the ocean mixed layer. Unavoidably, in dealing with this, we release momentarily the coastal-only perspective (but most of what we will say is valid also there), only to converge again when going into the details of specific processes. Also, although the number of specific examples at a limited scale may be less abundant than in the previous section 3.1, they will also show the crucial role of the full coupling in some of the most dramatic situations.

3.2.1 - The physics of the ocean mixed layer

where we provide the basic physics that govern the upper layer of the ocean and the interaction with the atmosphere, all modulated by waves

The depth of the ocean mixed layer, also known as the ocean surface boundary layer (OSBL), is maintained by a number of processes, including most importantly buoyancy production through heating and cooling and shear production. It is however clear now that waves also play a role in the mixing of near-surface waters. 
3259 Breaking waves and whitecaps are the most visible manifestation of mixing close to the sea surface 3260 (Monahan, 1971; Wu, 1979; Scanlon et al., 2016). They increase the turbulence in the upper part of 3261 the ocean significantly (Craig and Banner, 1994; Craig, 1996, Gemmrich et al, 1994, Gemmrich and 3262 Farmer, 1999) and play a crucial role in homogenizing the uppermost part of the OSBL to a depth of 3263 the order of the significant wave height.

3264 Through the interaction with the Coriolis effect, the Stokes drift velocity (Stokes 1847) associated 3265 with the wave field adds an additional term to the wave-averaged momentum equations. The effect 3266 was first presented by Hasselmann (1970) and has since been investigated for idealized cases by 3267 Weber (1983), Jenkins (1987), McWilliams and Restrepo (1999) and McWilliams and Sullivan 3268 (2000), among others. The force is known as the Stokes-Coriolis force or the Hasselmann force 3269 depending on whether it is considered to be purely an effect of the average Coriolis force acting on a 3270 particle with a Lagrangian velocity as given by the mean currents and the waves, or as a tilting of the 3271 planetary vorticity (Polton et al, 2005; Broström et al, 2014). The force does not directly modify the 3272 total mass transport, but it alters the distribution of momentum over the depth of the Ekman layer 3273 (McWilliams and Restrepo, 1999; Polton, 2009).

3275 The Stokes drift decays rapidly with depth, but near the surface it can reach values close to $0.7 \mathrm{~m} \mathrm{~s}^{-1}$. 3276 The full two-dimensional spectrum is in principle required to compute the Stokes drift velocity 3277 profile (Janssen et al., 2004; Janssen, 2012), but many simplified profiles (most commonly the 3278 monochromatic profile) are often used (see, e.g., Skyllingstad and Denbo, 1995; McWilliams and 3279 Sullivan, 2000; Carniel et al., 2016; Polton et al., 2005; Saetra et al., 2007; and Tamura et al., 2012). 3280 However, this underestimates the near-surface shear and overdoes the deep Stokes drift (Ardhuin et 3281 al., 2008). Recently, profiles that improve the shear and the deep flow still relying on the same 3282 integrated parameters (mean period, first order spectral moment and surface Stokes drift) have 3283 recently been proposed (Breivik et al., 2014, 2016).

3285 The interaction between the wave-induced Stokes drift and planetary vorticity leads to Langmuir 3286 circulation, as was shown by Craik and Leibovich (1976). Skyllingstad and Denbo (1995) and 3287 McWilliams et al. (1997) were the first to identify the significant role of Langmuir turbulence in 3288 enhancing mixing in the upper ocean. Several studies have employed large eddy simulations (LES) to 3289 investigate the impact of Langmuir turbulence in the upper ocean (Skyllingstad and Denbo, 1995; 3290 McWilliams et al., 1997; Teixeira and Belcher, 2002; Polton and Belcher, 2007; Harcourt and 3291 D'Asaro, 2008, Grant and Belcher, 2009), and in some cases even direct numerical simulations 3292 (DNS) have been employed. Most of these studies have found and confirm that waves do have a 
rather profound impact on the upper part of the ocean, but there is still considerable disagreement about which processes are more important. So far there have been few studies (see below) of the wave impact on three-dimensional ocean circulation models or fully coupled models of the ocean, the atmosphere and the oceanic wave field although the potential impact of waves on the climate system is recognized (Babanin et al., 2009; Cavaleri et al., 2007; Fan and Griffies, 2014, Li et al, 2015).

All these results have shown that wind-generated gravity waves have a profound effect on the OSBL, and may help explain the insufficient mixing found in Eulerian ocean models, especially in the extratropics. Langmuir turbulence, Stokes-Coriolis forcing and the direct injection of turbulence by breaking waves may substantially reduce the common shallow bias in the mixed layer depth predicted by most state-of-the-art climate models (Fan and Griffies, 2014). Furthermore, waveturbulence interaction directly affects the evolution of weather systems and thus the predictability of forecast models (Breivik et al, 2015).

While the reality of the above mentioned processes is now qualitatively undisputed, having experimental proofs in the field is another matter. Indeed observing wave-induced turbulence is challenging for two distinct reasons. First, obtaining measurements of turbulent parameters in the wave zone means either placing instruments in the violent environment of breaking waves, or remotely measuring quantities in the wave-breaking zone. Secondly, the spectral gap between turbulent and wave-related scales is small, meaning that wave motion may easily be mistaken for turbulent activity. New instruments and methods for making Eulerian and Lagrangian measurements within the wave-breaking zone of the mixed layer are now becoming available. Examples of such instruments are the MATS turbulence profiler (Fer and Bakhoday-Paskyabi 2014) and the buoyant Air-sea Interaction Profiler (ASIP; see Ward et al, 2014). The SWIFT buoy used by Thomson (2012) appears as a promising solution for also the Arctic waters.

Of course theoretical estimates of the energy input into turbulence exist. As for Stokes drift and Langmuir circulation we use the wave spectrum, we can use the energy balance equation of the third generation wave models (Komen et al., 1994) to evaluate the momentum and turbulent kinetic energy fluxes into the ocean as respectively

$$
\tau_{o c}=\tau_{a}-\rho_{w} g \int_{0}^{2 \pi} \int_{0}^{\infty} \frac{\mathbf{k}}{\omega}\left(S_{i n}+S_{d s}\right) d \omega d f
$$

and 
$\Phi_{o c}=-\rho_{w} g \int_{0}^{2 \pi} \int_{0}^{\infty}\left(S_{i n}+S_{d s}\right) d \omega d f$

3325 hence directly from source terms.

3326 The interplay between waves, ocean and atmosphere has different facets. While growing waves 3327 absorb energy and momentum from the wind field, in turn they release it when they break (Janssen et 3328 al., 2004; Rascle et al., 2006; Ardhuin and Jenkins, 2006; Janssen, 2012). This lowers or raises the 3329 stress on the water side (i.e., the stress below the oceanic wave field) relative to the air-side stress, 3330 depending on whether the sea state is growing or decaying. Only when the wave field is in 3331 equilibrium with the energy injected by the wind will the stress on the two sides of the surface be 3332 equal.

While wave breaking affects and mixes the upper few meters of the ocean, we still face (see above) the problem of a not sufficiently deep mixed layer in most of our model results. Indeed ocean models tend to mix too weakly (or have ad-hoc mixing parameterizations that actually overdo the mixing, see Breivik et al, 2015), producing warm biases which affect their heat uptake and deep-water formation (Babanin et al, 2009, Huang et al, 2011, Fan and Griffies, 2014). When coupled to meteorological models, this warm bias in turn affects the atmosphere by distorting the atmospheric deep convection and thus upsetting the delicate feedbacks in the climate system (Sheldon and Czaja, 2014). In the study by Fan and Griffies (2014) a significant change in the mixed layer temperature and its vertical extent was achieved with the introduction of Langmuir turbulence following the parameterizations by McWilliams and Sullivan (2000) and Smyth et al. (2002), as well as the parameterization of mixing by non-breaking waves suggested by Qiao et al. (2004). This latter mixing process appears similar to the mixing due to the high Reynolds numbers of the orbital motion of non-breaking waves explored by Babanin (2006) and Babanin and Haus (2009). The Qiao et al. (2004) approach is not yet universally accepted, but it is relatively easy to implement and has been used for a range of regional and global model experiments where it is found to make an impact. Using a climate model of intermediate complexity, Babanin et al. (2009) explored the effect of the three wave-related mixing processes, namely injection of turbulent kinetic energy from breaking waves, Langmuir circulation and the aforementioned mixing by non-breaking waves. They found that all three processes contribute to the depth and temperature of the mixed layer. Similarly, Huang et al. (2011) coupled WAVEWATCH III (Tolman et al., 2002) to a version of the Princeton Ocean Model (POM, Blumberg and Mellor, 1987) and demonstrated an improved summertime temperature profile using the non-breaking parameterization of Qiao et al. (2004). 
3357 Waves affect all the processes at the interface, and in particular the exchanges between ocean and atmosphere. These include aerosol production, heat transfer, radiation, albedo (and this is not an exhaustive list). Janssen and Viterbo (1996) showed how sensitive the atmosphere and its evolution can be to the sea albedo that is heavily affected by the presence of breaking waves.

Although relevant for climate, hence on very large scales, much of what said holds also for enclosed seas. Moreover, the smaller the spatial scale we consider, the shorter the involved time scale, hence more rapid the effects. This is particularly true when approaching the coastline in shallow water. A classical case, that we will illustrate with a couple of examples, is the combined wave set-up, surge and increased surface stress when wind and waves encounter a shallow coast. The bottom-induced breaking leads to wave set-up (see the example in Section 3.1 and in Figure 3.9), while the dynamics of the basin and the local surface wind stress pile up water against the shore. Note that approaching the shore, because of momentum balance, the external forcing (radiation stress and surface wind stress) must be counterbalanced on smaller depth, which implies steeper sea level gradients towards the shore. Besides the rough surface due to wave breaking increases the surface drag, leading to an increased overall effect of the, albeit slightly reduced, wind speed. Of course this is reflected in a positive feed-back on the waves and the surge.

\subsection{2 - Implementations with wave parameterizations}

illustrative examples of where in the world the just described couplings have been applied

Having presented the main (but certainly not exhaustively) aspects of the physics involved in the full coupling of the ocean interior, surface waves and the atmosphere (with a keen eye on wave modeling), it is now time to present an overview of where and how such coupled systems have been applied for enclosed seas .

Several one-dimensional mixed layer models have appeared that incorporate wave-mixing parameterizations, in particular Stokes production and wave breaking (Rascle et al. 2006; BakhodayPaskyabi et al. 2012, Janssen, 2012). Kantha and Clayson (2004) revised the second-moment turbulence closure model to account for Langmuir Circulation (LC) effects by adding the Stokes drift production term to the TKE equations, and Harcourt $(2013$, 2015) developed a second moment closure model of Langmuir turbulence.Despite extensive numerical investigations of Langmuir circulation and its turbulent mixing based on Large Eddy Simulations and one-dimensional ocean 
vertical mixing models, only a few studies have coupled and tested LC, breaking, and non-breaking wave effects on three-dimensional ocean models, let alone coupled models of the atmosphere and ocean (Li et al, 2015, 2017). Fan and Griffies (2014) implemented and assessed the impact of parameterized wave-induced mixing on global climate simulations. Li et al (2015, 2017) incorporated WAVEWATCH-III into the Community Earth System Model (CESM) and found that implementing Langmuir turbulence improved the shallow bias of the mixed layer depth in long climate integrations. Recently, Breivik et al. (2015) implemented a fully coupled atmosphere-waveocean global numerical weather prediction model by coupling the operational ECWAM wave model (Janssen, 2004; Bidlot, 2012) to the NEMO ocean model component as part of the ensemble suite of the Integrated Forecast System (IFS) of ECMWF. They implemented mixing by wave breaking, Stokes-Coriolis forcing and wave-modulated stress in their model, and demonstrated the vital role of correctly including wave mixing for reducing the SST bias.

The wave-related processes described above are quite varied in their impact as well as the complexity involved in their implementation in ocean models. Two model systems in particular have been used to test these processes, namely the Regional Ocean Model System (ROMS, see Shchepetchkin et al, 2005, Warner et al, 2008 and Warner et al, 2010) and the Nucleus of European Modelling of the Ocean (NEMO) model (see Madec et al, 2012). Other model systems also in regular use include POM (Blumberg and Mellor, 1987). ROMS has been extended to incorporate the vortex-force formalism by Uchiyama et al (2010) and through the COAWST coupled WRF-SWAN-ROMS setup it has been used extensively for near-shore applications in which wave effects play an important role (Kumar et al, 2011, 2012). An implementation of the Stokes-Coriolis force in integrated form (impulse) was presented by Röhrset al (2012).

The NEMO model has also recently been extended (see Breivik et al, 2015, Mogensen et al, 2017) to include Stokes-Coriolis forcing as a body force in the momentum equations, a modification of the flux of turbulent kinetic energy following Craig and Banner (1994) and Mellor and Blumberg (2004), but with fluxes estimated from the EC-WAM wave model component of the forecast system of ECMWF (Janssen, 2004). The momentum flux from the atmosphere to the ocean is also modified by the wave field. The impact has been found to be quite significant regionally, up to $0.5 \mathrm{~K}$, caused by the Stokes-Coriolis force and the momentum flux. The injection of turbulent kinetic energy has an even greater effect, although this is in part due to the fact that NEMO has too vigorous mixing in its default setup, and the impact when compared to a law-of-the-wall boundary condition is on the order of $0.5 \mathrm{~K}$ in the extra-tropics. Recently, Alari et al (2016) have shown that the impact of these three 
3424 wave effects is also significant in enclosed seas. In the case of the Baltic Sea the impact on upwelling 3425 and downwelling from Stokes-Coriolis forcing reached $0.3 \mathrm{~K}$. The impact of the wave field on water 3426 level has also been found to be significant in storm events in the North Sea. Here the effect is mainly 3427 due to the increased stress from growing waves (rougher sea surface). This leads to enhanced drag 3428 and with it increased water level. This was first demonstrated by Mastenbroek et al (1993) and 3429 recently demonstrated for two intense storms in the autumn of 2013 by Staneva et al (2017) using a 3430 regional NEMO model.

3432 Are we in the position to draw a solid conclusion on the basic physics of ocean, waves and 3433 atmosphere interactions? The situation is not yet crystal clear. Although as we have seen quite a 3434 number of studies have addressed the modeling and parameterizations of the coupled system 3435 (Babanin et al, 2009; Fan and Griffies, 2014; Breivik et al, 2014; 2015), it is still unclear which of the 3436 wave-related effects are the most important for the oceanic mixed layer. Recent investigations of the 3437 mixed layer depth biases have revealed that surface gravity waves do deepen the thermocline. The 3438 question is how. Wave-turbulence interaction, and in particular Langmuir mixing, breaking waves, 3439 and non-breaking gravity waves (Qiao et al, 20014; Babanin 2006; Babanin et al, 2009; Huang et al, 3440 2011) all appear to be candidate mechanisms for explaining the lack of mixing. These processes 3441 enhance the turbulent kinetic energy throughout the mixed layer and thus influence both the depth of 3442 the mixed layer and the near-surface temperature (Janssen, 2012, Breivik et al, 2015). Most likely all 3443 of them act in this direction, but their relative importance remains to be determined. It appears clear 3444 that more work is needed to get a proper representation of wave-induced effects in ocean models.

\subsection{3 - Practical examples}

cases where the coupling turned and remain essential for the correct results

3448 As in Section 3.1 we provided examples for the wave-current interaction, we describe here a few 3449 cases where the final results imply a more or less direct interaction with also the atmosphere.

- Upwelling in the Baltic Sea

3452 Towards the end of the previous section we cited how in the Baltic Sea (Alari et al., 2016) full 3453 consideration of the impact of the wave effects on the local circulation and atmospheric modeling led 3454 to a manifest upwelling in the coastal areas. The corresponding results are shown in Figure 3.11. 3455 Panel a) shows an upwelling, documented by a Modis image, off the coast of Estonia. Panel b) is the 3456 control run without the coupling with waves and the atmosphere. The role of coupling is evident in 3457 c), with a much stronger upwelling, much more similar to the one detected by the satellite. 

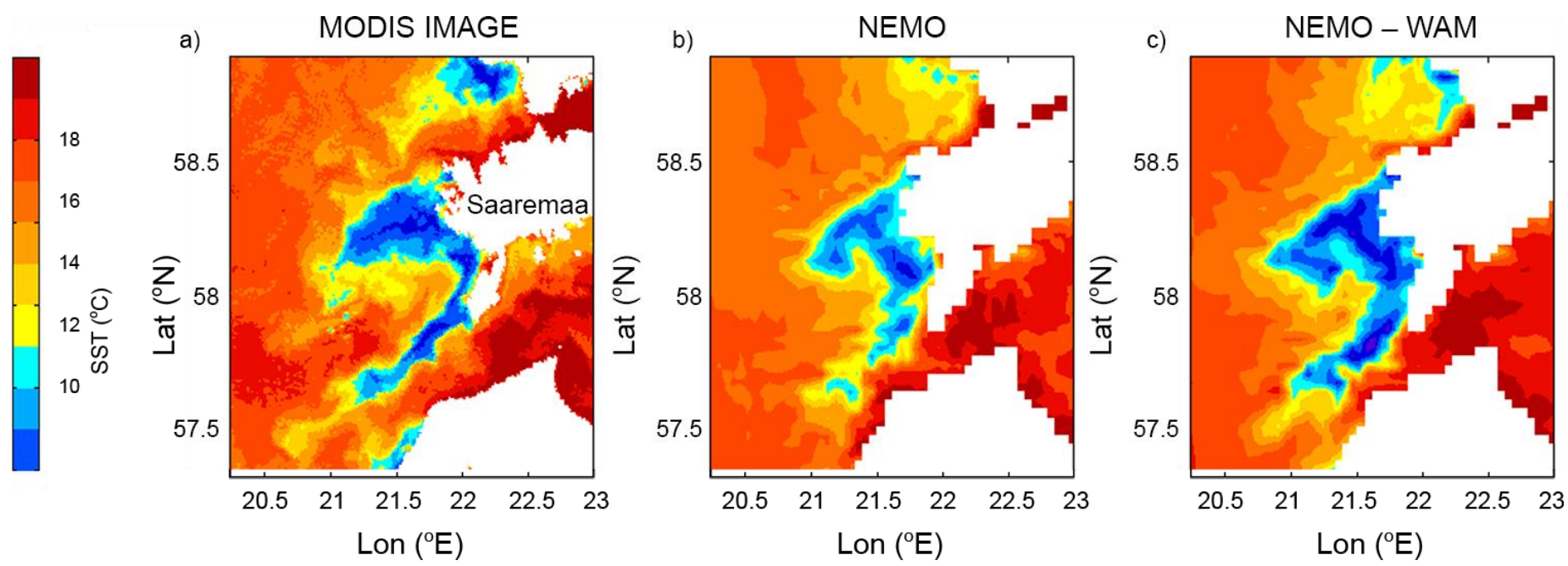

Figure 3.11 - Upwelling in the Baltic Sea, off the coast of Estonia. Panel a) satellite Modis image, b) and c) uncoupled and fully coupled runs respectively.

- Deep sea water formation due to cold outbreak

3464 In the winter of early 2012 an exceptional inflow of very cold air hit the Northern Adriatic Sea 3465 (Figure 2.3, panel b) with sustained north-east bora wind for more than one week. This gave the 3466 chance to investigate how, perhaps counter-intuitively; waves can play an important role in the 3467 definition of also deep water properties. In the Adriatic Sea the process is known. Therefore the 3468 forecast and the duration of the event led to the organization of a devoted campaign that, together 3469 with the oceanographic tower "Acqua alta" (panel d), provided a wealth of detailed and distributed 3470 data. At the same time a full two-way coupled wave, atmosphere and ocean model system was 3471 implemented using, and comparing the results with, all the measured data. Improvements in the 3472 turbulent heat fluxes forecast using the atmosphere-ocean coupled run were related with the dynamic 3473 sea surface temperature brought into the system by the ocean model, while full coupling with also 3474 surface waves further improved the simulations and the results with respect to the in-situ measured 3475 data acquired at the tower. Benetazzo et al. (2014) and Carniel et al. (2016) provide a full description 3476 of the model set-up and related results. The coupling turned out essential from different points of 3477 view. Coupling waves and currents increased the latter by $0.15 \mathrm{~ms}^{-1}$, in so doing slightly reducing the 3478 wave heights. At the same time the enhanced (because of waves) turbulent heat fluxes $\left(+45 \mathrm{Wm}^{-2}\right.$, 3479 about $10 \%$ of the average heat budget) led to an increased turbulence in the atmospheric surface 3480 layer. In turn (Abdalla and Cavaleri, 2002) the higher gustiness level led to a more effective and rapid 3481 growth of the wave field that, via the strong breaking, was fed again to the current. The evolution of 3482 the mean kinetic energy in the basin confirms that the explicit inclusion of wave effects in 3483 atmospheric-ocean interactions provide a different dynamical characterization of the overall basin. 
3484 Coupling ocean, atmosphere and waves (at the limit also in only a sub-region of the model domain) 3485 may significantly modify the water circulation and characteristics in much larger areas in spite of 3486 their apparently small extent of influence. This can strongly affect the volume of water involved in 3487 the cooling process within a whole semi-enclosed basin, its density and kinetic energy, conditioning 3488 its potential contribution to deep-sea ventilation. In the event of winter 2012 the cold water produced 3489 in the northern part of the basin moved southwards in a narrow flow along the Italian peninsula, till 3490 reaching in the southern part the deep part of the basin, flowing then out through the Otranto Strait 3491 into the Ionian Sea (Figure 2.3) to become part of the local deep water.

3493 - Cyclogenesis

3494 A similar process, but on different scale and depth, happens especially in autumn in the north-western 3495 part of the Mediterranean Sea (Figure 2.3, panel a). In this period of the year the first northerly 3496 storms bring cold energetic air over the still warm local water. Granted the differences between less 3497 (Northern Adriatic) and more deep (Gulf of Lyon) conditions, the process is similar to what just 3498 explained in the previous example. Very strong waves enhancing the current, very large turbulent 3499 heat fluxes with consequent gustiness (the local mistral winds are famous for their violence), hence 3500 increased active generation, more white-capping, feed-back on current, etc. Of course there is a strong cooling of the surface water and heating of the atmosphere. This is where, given the scale, the main difference arises. The strong heating of the lower layers of the atmosphere leads to the generation of an intense low pressure system (cyclogenesis) with further implications for the local wave and current fields. Incidentally, quite often these low pressure systems are the ones that give rise to the conditions favorable to the Venice floods in the Adriatic Sea (see below).

- Medicanes

The high surface temperatures of the Mediterranean Sea can lead to the formation of intense low pressure systems with some of the characteristics of hurricanes, hence the name assigned to these systems: Medicanes. Although not particularly intense in terms of minimum central pressure, the associated wind speeds can be rather strong. A particularly intense one occurred between 4 and 7 November 2011 in the western part of the basin. The minimum pressure was estimated at $985 \mathrm{hPa}$ with wind speeds up to $27 \mathrm{~ms}^{-1}$. Ricchi et al. (2017, submitted) have done a careful analysis of the 3514 system. In particular they explored in detail how sensitive the results were to 1) the coupling, first 3515 between ocean and atmosphere, then also the inclusion of two-way wave effects, 2) different 3516 parameterizations of the sea surface roughness. The positive SST anomaly $\left(+1.5-3^{\circ} \mathrm{C}\right)$ in the area 3517 turned out essential in the development of the cyclone and in its evolution. This was crucially 
3518 dictated, in the model results, by the SST evolution and consequently by the level of coupling. The 3519 comparison of the SST recorded on a buoy in the Gulf of Lyon shows clearly that the best result (i.e. 3520 the most intense cooling during the Medicane) was achieved when taking into account the increased 3521 heat transfer from the sea to the atmosphere due to the presence of waves. This corresponded also to 3522 the lowest modeled minimum pressure. Notwithstanding the increased strength of the system, this did 3523 not lead to also a further increase of the estimated wind speeds because of the increased friction at the 3524 surface. However, the combination led to larger wave heights.

A remarkable and instructive finding came from the use of three different parameterizations of the sea surface roughness, based respectively on wave steepness $H_{\mathrm{s}} / L_{\mathrm{p}}$ and (two) on wave age $u * / c_{\mathrm{p}}$. While the related expressions (see Ricchi et al., op.cit., for a full discussion) have been derived in what, relatively speaking, we can call 'normal conditions', the use in a hurricane may lead to contrasting results. The reason is the strong spatial variability of the wind and wave conditions, and consequently the possible step, rather unnatural, distribution of the results. Ricchi et al. (op.cit.) even suggest to make some preliminary tests in practical applications to verify which a parameterization may appear as the most suitable for the situation of interest. This strongly suggest we still have a long way to go before having a good physical description of the interplay between wind and waves.

\section{- Hurricane Katrina}

In the previous Section 3.1.4 we had shown, among other examples, the wave, wave set-up, and surge maximum conditions in a large area around and to the east of the Mississippi delta. It is this last field (Figure 3.8, panel c) that we recall to illustrate in a dramatic case one aspects of the physics we described at the end of Section 3.2.1. It is hard to imagine the wave conditions in the shallow zone just east of the delta, where maximum wind and waves pushed waters towards the coast. The crucial point is the roughness of the sea surface, the consequent increase of the surface wind stress, the increased local depth (surge), hence the higher waves closer and closer to coast and 3544 in the previously dry land.

- Venice flood

3547 In one of the examples in Section 3.1 we have shown the wave set-up during the 1979 storm whose 3548 flood ranks second in recorded history (since 1872). The number one was 4 November 1966. No measured data exist because the tower in Figure 2.3, d was not yet there and all the tide gauges at the coast were destroyed (as most of the jetties). The official level was recorded as the floating mark left on the walls of Venice. Cavaleri et al. (2010) made a careful reconstruction of the event. The 
3552

(1)

resulting profile of the sea level at the peak of the storm along the whole basin is in Figure 3.12. The dramatic increase of the level when moving in shallower and shallower water, when approaching the northern end of the basin, is clear. Note that also here the target at the left was the entrance to the lagoon, the relevant information for Venice. Two kilometers further on, at the actual beaches, the level was estimated at least $0.5 \mathrm{~m}$ higher.

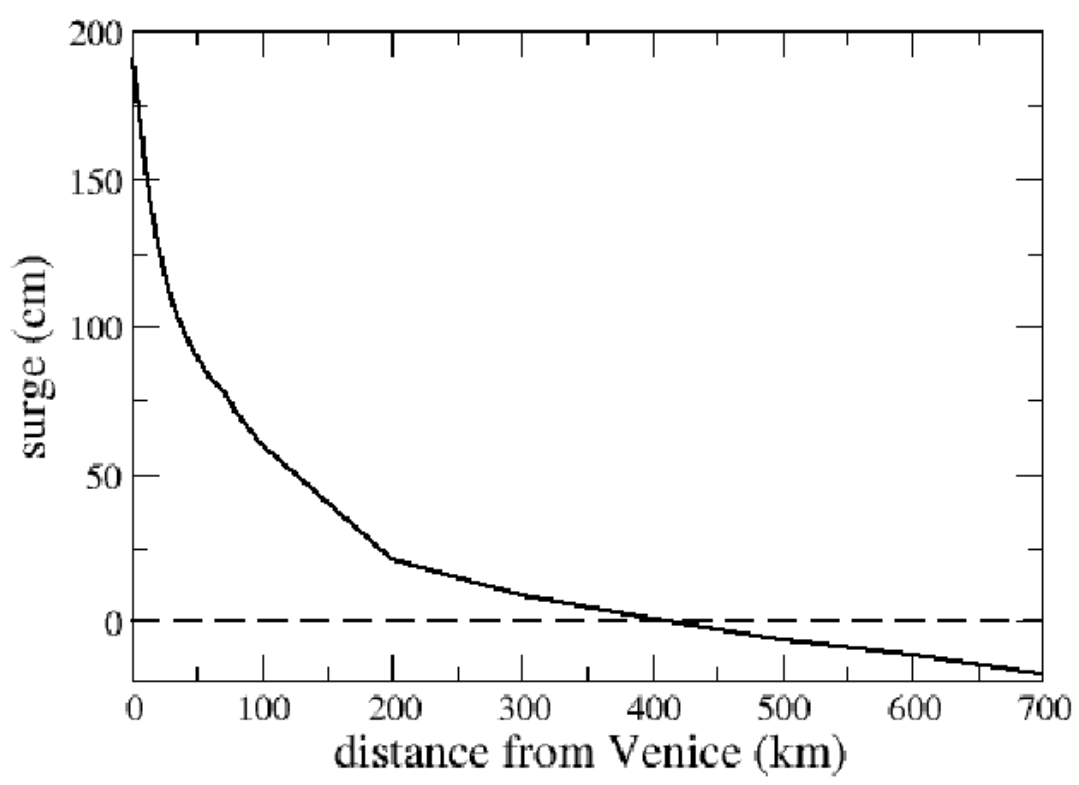

Figure 3.12 - Surge along the Adriatic Sea (see Figure 2.3, panel b) at the peak of the storm of 4 November 1966. The horizontal scale shows the distance from the Venice coast. The estimate is $2 \mathrm{~km}$ offshore (6 m depth), at the end of the jetties bordering the entrance to the lagoon.

\section{- Unbalance in the Venice lagoon}

Remaining in the same area, the Venice lagoon (Figure 2.3, panel c) is $50 \mathrm{~km}$ long, $10 \mathrm{~km}$ wide, very shallow, with an average depth of slightly more than one meter, with sparse deeper canals. When the north-east bora wind blows with high speed, the water at the southern end of the lagoon, where a small town is located, is about one meter higher than in Venice. The reason is again the interaction of the short choppy waves with the atmosphere. In the lagoon the bora-induced waves are permanently very "young", with vigorous breaking. Consequently there is a high drag, hence the pile-up at the southern end. This notwithstanding the (partially) reduced wind speed (because of the enhanced surface drag) as proved by direct comparison with the parallel data recorded at the external station close to the sea and most of all those from the oceanographic tower. 
J.Portilla, S.Abdalla, J.-R.Bidlot, Ø.Breivik, L.Cavaleri

jportilla@ymail.com

\section{4 - Wave data assimilation in enclosed seas}

where we outline the need for an effective and optimized data assimilation system in the coastal and enclosed seas, and we describe, on the base of the available information, the best approach for both long term and operational forecast activity

\section{1 - The situation to deal with}

where, before going into the actual methodology, we frame the problem to be faced

We should never forget that the models we love and use so much are always, and still are, just models. Irrespective of the recent model improvements, they are still far from perfect. As such they provide estimates of the quantities of interest, estimates that by definition are prone to errors. Where possible, we make use of the corresponding measured values, where and when available, to get from the two combined information (measured data are estimates as well, see Section 2.5) the best overall estimate we can get. This is essential both for long term hindcast as for the best picture of the situation to start from for the next forecast.

Having outlined the general idea, we soon find that the practical application faces different problems in the open ocean and in the enclosed and coastal seas. From a very general point of view there is no doubt that the performance of open sea modelling has outpaced the one where the coasts play their role (after all, this is the reason for this paper). There are many reasons for this. The orography affects the local meteorology, hence the wind fields we depend upon (see Section 2.1). The geometry of the basin, limited depths, coastal currents, stronger coupling, etc., all contribute to make the task more complicated with likely greater errors. On the other hand this, the coastal environment, is where most of our interests are concentrated. Both these factors highlight the need for an approach capable to optimize the available information making use of the best practical and theoretical approaches. Of course this leads us to data assimilation as the natural approach to achieve this.

Open ocean, in practice global, wave modelling has made use for a quarter of a century of the information available via satellite altimetry. Within its limitation of providing only the $\mathrm{H}_{\mathrm{s}}$ integral parameter, the ubiquitous information from altimeters has played, and still plays, a fundamental role in improving the quality of our model results in the open oceans. However, some of the 
3613 characteristics of this ubiquity show their limit in enclosed seas. Figure 4.1 shows the performance 3614 of the ECMWF wave forecast system for different environments.
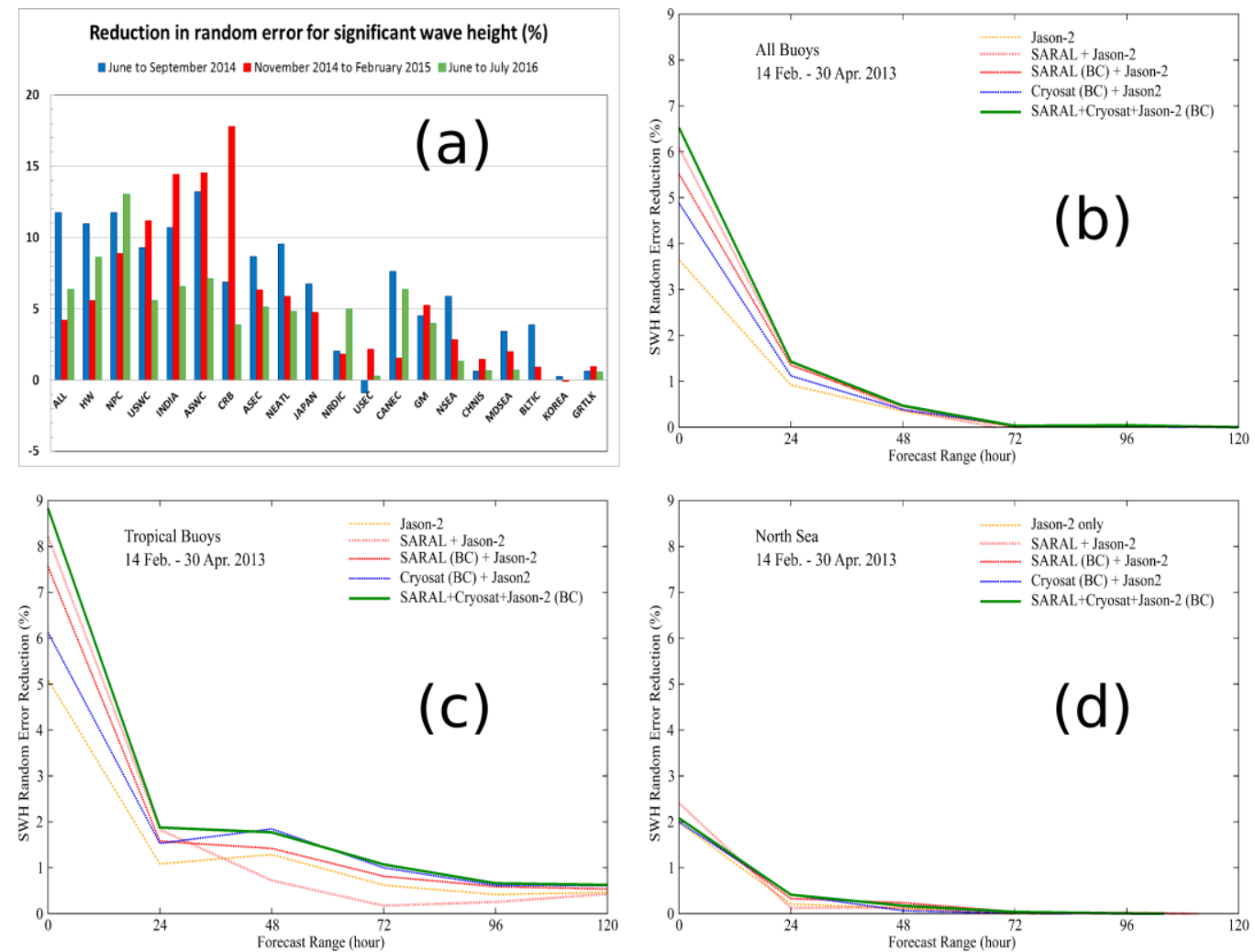

Figure 4.1 - a) Standard deviation error of the ECMWF model significant wave height. Reduction of error (positive indicates improvement) following assimilation of buoy data. The various areas are detailed in Table 4.1. Panels b, c, d show the reduction of standard deviation error when assimilating different altimeters data for all buoys, tropical areas, and the North Sea respectively.

In panel a there is an obvious difference between the wide open basins (e.g., INDIA and CRB) and the enclosed seas (e.g., MEDSEA and KOREA). The overall result is made even clearer in the other three panels, b, c, d, where we show the same results, but specified per altimeter, area and time. Panel $\mathrm{b}$ reports the performance on the globe (for all the available buoys), c only the tropical areas, d for a typical (North Sea) enclosed (but not so enclosed) basin. The different extent of the lasting information is evident. Of course this has to do with the time a wave system takes to cross a basin and with the dominance of wind sea and/or swell. However, a more fundamental role is played by the 'only integral parameter' information mostly available from altimeters and their discontinuous and selective passing over an area of interest. This becomes crucial in the coastal and enclosed sea environment. 


\begin{tabular}{|l|l|}
\hline ALL & All \\
\hline HW & Hawaii \\
\hline NPC & North Pacific Coast \\
\hline USWC & US West Coast \\
\hline INDIA & India \\
\hline ASWC & Australia South West Coast \\
\hline CRB & Caribbean Sea \\
\hline ASEC & Australia East Coast \\
\hline NEATL & North-East Atlantic \\
\hline JAPAN & Japan \\
\hline NRDIC & Nordic (North Atlantic and Norwegian Sea) \\
\hline USEC & US East Coast \\
\hline CANEC & Canadian East Coast \\
\hline GM & Gulf of Mexico \\
\hline NSEA & North Sea \\
\hline CHNIS & English Channel and Irish Sea \\
\hline MDSEA & Mediterranean Sea \\
\hline BLTIC & Baltic Sea \\
\hline KOREA & Korea \\
\hline GRTLK & Great Lakes \\
\hline & \\
\hline
\end{tabular}

3634 Panel 4.1.a shows the percent reduction in standard deviation error, following assimilation of $\mathrm{H}_{\mathrm{s}}$ 3635 data in different areas and basins. The reference corresponds to the ECMWF global wave model 3636 without any wave data assimilation. Three analysis experiments were carried out. 1) The periods 3637 from June to September 2014 and 2) from November 2014 to February 2015 assimilated Jason-2, 3638 Cryosat and Saral data and were based on the 40km global model. 3) For the period June-July 2016 3639 the $28 \mathrm{~km}$ model was used, assimilating also the Jason-3 data.

3641 Luckily another source of information is frequently available. Wave measuring buoys, still within 3642 the limitations outlined in Section 2.5, provide the most complete information presently available. 3643 Besides making available, if arranged for, the actual time series of the single waves at the 3644 measuring position, they provide also the full 2D spectrum with all the information that can be 3645 derived. Besides wave buoys are available (see, e.g., Figure 2.9) not only as large networks, but as 3646 specifically located instruments for a specific operational purpose wherever, e.g. harbours or 3647 operational rigs, the information needs to be available 'now and there'. Both the volume of the 3648 available information (at least two orders of magnitude more than the single integral parameter) and 3649 the crucial role of good quality information that is often required for specific applications, imply a 3650 very sound and complete approach to data assimilation, capable to use not only the latest available 3651 information, but also the one associated to the geometry and characteristics of the basin. This is 3652 what we describe in the following sections. 


\section{2 - State of the art}

where we point out the limitations of the most used approaches

In their traditional approach, altimeter and, where available, buoy data have been used only with their synthetic information of significant wave height. Of course this approach hides the true information, i.e. the complexity of the actual distribution of energy among the various frequencies and directions. While in the inner seas a 2D spectrum will display in general less wave systems (typically wind sea and swell) than in the ocean, we need to consider that the smaller the basin the stronger will be in general the spatial and temporal gradients that characterize the environment. Time gradients, i.e rapidly changing conditions (see Section 2.1), point to the need of a more detailed (in spectral space) and frequent information if this is to be provided to harbours or general users. Of course this pushes the choice towards devoted in situ measurements capable to provide the required full spectral (f- $\theta$ ) information. This approach, in most of the cases a buoy, has several advantages. First, a good knowledge of the local wave climate (that we can derive from previous modeling) allows to choose strategically the right monitoring position that, granted the modern technology, can continuously supply data in real time. Second, in situ instruments have more flexibility in the variables to be measured. Nowadays regions with high marine activity, hence requiring effective forecast systems, are often covered by dense monitoring networks (see, among others, Bertotti et al., 2014; Alfieri et al., 2012; Alves et al., 2014; Landsea et al., 2012). Therefore the potential exists for fully exploiting the available information to improve the inner sea and coastal (albeit short term) forecasts.

During the last decades several attempts have been made to implement wave DA routines in enclosed seas and nearshore scenarios. Good examples are provided by Voorrips et al. (1997), Siddons et al. (2009), Portilla (2009), Veeramony et al. (2010), Waters et al., (2013), Rusu and Soares (2015), Wahle et al. (2015), Panteleev et al. (2015. In most of the cases the data have routinely been assimilated using a simple Optimal Interpolation scheme with static error covariance specification (e.g., Abdalla et al., 2010). In general there are a couple of bottlenecks in these developments. The first one relates to the fact that improvements in DA are understood (or misunderstood) as adopting more complex optimization algorithms (e.g., 3DVAR, 4DVAR, Optimal Interpolation, Kalman filters). However, environmental DA in general has to deal with a more basic issue which is how to combine a full 2D background field with single point measurements. In practice in DA one has to produce first the full $2 \mathrm{D}$ observational field that is then 
combined with the background field by the optimization algorithm. This first task is usually embedded in the whole DA algorithm as it will be illustrated below. As mentioned above, the second limitation is that the actual model variable is the wave spectrum. The significant wave height (or wave period) is only a bulk parameter, with a two-dimensional matrix reduced to a single scalar, and this is a very drastic reduction of information. In fact, for a given $\mathrm{H}_{\mathrm{s}}$ there are infinite possibilities of energy distribution, and this produces uncertainties in both ways, first when obtaining the $2 \mathrm{D} \mathrm{H}_{\mathrm{s}}$ background field, and then when the analysed field is transferred to the analysed spectra. For DA in general, but also specifically for enclosed and coastal seas, a more complete information is required than what frequently used. We claim that only a fully spectral information can satisfy the practical requirements of coastal and inner seas managements and engineering. It is in this direction that we concentrate our effort in this chapter. Here we advocate for a DA algorithm consistent with the modern third-generation spectral wave models and spectral observing techniques. This is explained in the following section.

\section{3 - Towards the development of a spectral data assimilation scheme}

where we outline the best approach for the problem of interest

In a Bayesian framework, data assimilation is the process of combining modelled and observed data in such a way that the resulting output is more accurate than the two single or simply combined sources (see e.g., Evensen, 1994). A good wave DA system must be consistent with (a) the statistical properties of both observations and model output, (b) the model dynamics, and (c) the local wave conditions. These requirements are explained in more detail below.

(a) By statistical properties of the data, we mean their associated errors. We typically tend to regard observations as the ground truth, against which model is evaluated. In fact, observations can also 3712 be, and usually are, subject to different type of errors, like measuring errors as such, or instrument misrepresentation of the physical phenomena, or shortcomings of the methods for processing the signals, among others (see Section 2.5 for an extensive discussion). So more appropriately, measurements have to be regarded as a mere estimate of the truth with an associated standard deviation, exactly how model data have to be regarded (e.g., Janssen et al., 2007). Under this premise, the task of DA is statistically simple (in principle at least), and consists of producing a single output from the corresponding inputs and their (model and measurements) associated errors (e.g., Daley, 1993). 
3721 (b) For a DA system not to be too cumbersome, it needs to run along harmonically with the model 3722 dynamics. To achieve this, a few things need to be taken into account. The first is that the model 3723 variable to work with is not the significant wave height $H_{s}$, but actually the directional wave 3724 spectrum. Failing to consider this puts us in the very intricate situation of having to correct with a 3725 single parameter a variable that has typically a few hundreds degrees of freedom (typically $n \theta=24$ to 372636 directions and between $\mathrm{nf}=25$ and 36 frequency bins). In doing so, we are most likely forced to 3727 make assumptions that override the physics represented by the model (e.g., Lionello et al., 1992). 3728 The second important consideration is related to the model structure. To avoid numerical instability, the advection terms in the action balance equation are run with a time step that is consistent with the speed of waves over the computational grid. On the other hand sink and source terms run sequentially, and with a different time step tuned to the typical rate of change of the parameterized physical processes (e.g., Komen et al., 1994, and Holthuijsen, 2007). From this perspective, DA corrections behave to the model precisely as a sink-source term, so they have to be operated 3734 accordingly.

(c) A DA scheme can only be effective if it takes into account the physical processes involved in the wave evolution and propagation. In general, the wave climate can be characterized by the local wind-sea conditions and swells from distant storms. However, in enclosed seas, due to the limited dimensions of the basin, the distinction is often not so neat as in the open ocean. The time variability, recurrence, and magnitude of events are also fundamental aspects. In enclosed seas, wave conditions are typically low or moderate, but extreme events can be very destructive. They are episodic with recurrence generally associated to specific seasons or meteorological conditions (e.g., winter storms). Therefore, a precise knowledge of the local wave climate in terms of the 2D spectrum should be the starting point.

The large spatial gradients of wind and wave fields often found in enclosed seas, and in other specific near-shore scenarios, add other challenging features for local wave modelling. These large gradients arise for instance from very irregular bathymetries and topographies, with offshore, slanted, or funnelled winds (see Section 2.1). In this regard, it is important for the DA system to capture the geographical details of covariances between variables in complex topography, otherwise we would be trying to correct data at remote positions with unrepresentative measurements (Kalnay, 2003, pp 156-157). These requirements are hard to assess when only integrated parameters of the spectrum are considered. 
3755 Finally it is worthwhile to mention that, for all the reasons till now discussed in this paper, it is 3756 frequent o have systematic errors in enclosed sea wave modelling. It is especially in these case that DA becomes an essential tool to improve the quality of the results and, last but not least, to help analysing the situation.

We illustrate some of these aspects by analysing the main components of a generalized 3DVAR algorithm, in which the DA task is set-up as an optimization problem, where the differences between model and observations are to be minimized (e.g., Kalnay, 2003, eq 5.5.8). This is expressed by the set of equations from 4.1 to 4.3 .

$$
\nabla J\left(S_{f, \theta}\right)=\mathbf{B}^{-1}\left(S_{f, \theta}-S_{f, \theta}^{B}\right)+\mathbf{H}^{\mathbf{T}} \mathbf{R}^{-1} \mathbf{H}\left(S_{f, \theta}-S_{f, \theta}^{B}\right)-\mathbf{H}^{\mathbf{T}} \mathbf{R}^{-1}\left\{S_{f, \theta}^{O}-\mathbf{H}\left(S_{f, \theta}^{B}\right)\right\}
$$

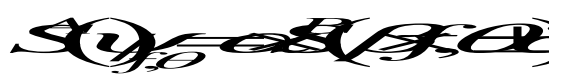

where $J$ represents the cost function to be solved, $S$ is the wave spectrum, $f$ and $\theta$ indicate that the differences are evaluated over the discrete spectral domain of frequency and direction, the superscript $B$ indicates background (model) grid points corresponding to both observation and remote grid points inside the assimilation domain, $O$ refers to observations, and $A$ to analysis $\left(S_{f, \theta}^{A}\right.$ $\left.=S_{f, \theta}(\psi)\right)$, where $\psi$ is the set of tuning parameters, composed of $\alpha, \beta$ and $\delta$, with the aim of correcting energy, frequency (or period), and direction respectively. $\mathbf{H}$ is the observation operator, which interpolates and transforms gridded model variables to observed variables. $\mathbf{R}$ is the observation covariance matrix, considered diagonal here, meaning that observations errors are uncorrelated. In turn B defines the weighting factors over the spatial domain computed from the error correlations associated to $S_{f, \theta}^{B}$ (namely, the background error covariance matrix). In this scheme, the two most important components of the system are $\psi$ and B. $\psi$ indicates how the model spectrum is operated in order to obtain the "analysed" spectrum. B relates to the weight the single observations have at the remote surrounding points inside the assimilation domain.

3785 Dealing with all the ( $\mathrm{nf} \times \mathrm{n} \theta)$ components of a 2D spectrum is not practical. A solution is suggested 3786 by the fact that these components are not independent. Indeed the wave conditions at given point 


$$
\rho_{i, j}=\frac{\left\langle\sigma_{i} \sigma_{j}\right\rangle}{\sigma_{i} \sigma_{j}}
$$

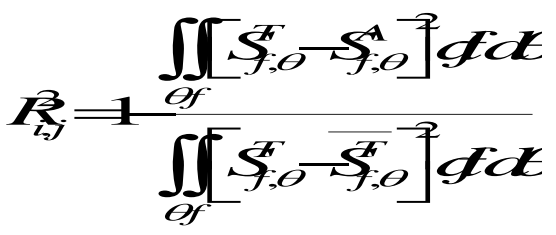


3817 Here the angle brackets indicate the error covariance between the assimilation point $i$ and the 3818 remote point $j, \sigma$ are the error standard deviations, and $\rho$ is the error correlation coefficient. A 3819 typical approach is to use a sufficiently long hindcast (one or better a few years) of the spectral 3820 wave conditions in the area of interest to derive the information to be then used in operational 3821 forecast and DA. The error standard deviation in (4.4) can be estimated for instance by comparing 3822 independent wave data sets, e.g. via the triple co-location method, as shown, among others, by 3823 Janssen et al., 2007). $R^{2}$ is the coefficient of determination, the numerator quantifies the differences 3824 between the true spectrum $T$ and the analysed spectrum $A$. The denominator is the normalizing 3825 factor in terms of the true spectrum. This procedure is illustrated with examples in the North Sea 3826 and Lake Michigan given in the two following sections.

\section{4 - Examples}

where we provide two rather different, both representative, examples of the approach we suggest as the best solution

\section{The North Sea case}

3834 The North Sea is a semi-enclosed basin about 1000x500 km across. It is widely open from the north flank to the North Atlantic, so swells originated in that area regularly enter the domain. Northerly winds are also very frequent, therefore the northerly wave systems are typically characterized by a mixture of locally generated waves and swell (Reistad et al., 2011; Aarnes et al., 2012; Semedo et al., 2015). Extreme conditions related to northerly waves might occur in combination with low pressure meteorological fronts leading also to storm surges. At its southwest corner, the North Sea is connected to the Atlantic through the English Channel. However, the channel is too narrow and partly shallow for ocean swells to penetrate, but the geography allows south-westerly winds to blow over a relatively long fetch, and consequently waves with this direction are also important in the domain. Other less frequent situations can be found specially related to wind blowing from different 3844 directions. In socio-economical terms, the southern North Sea is a very active region and hosts important maritime activities such as oil extraction and harbours as Rotterdam (see Section 2.6.3), Antwerp, and London. Waves in the area are forecast by different centres, and they are extensively monitored with buoys and radars networks (see,, among others, Wolf et al., 2011, Maresca et al., 3848 2014, Behrens, 2015). 

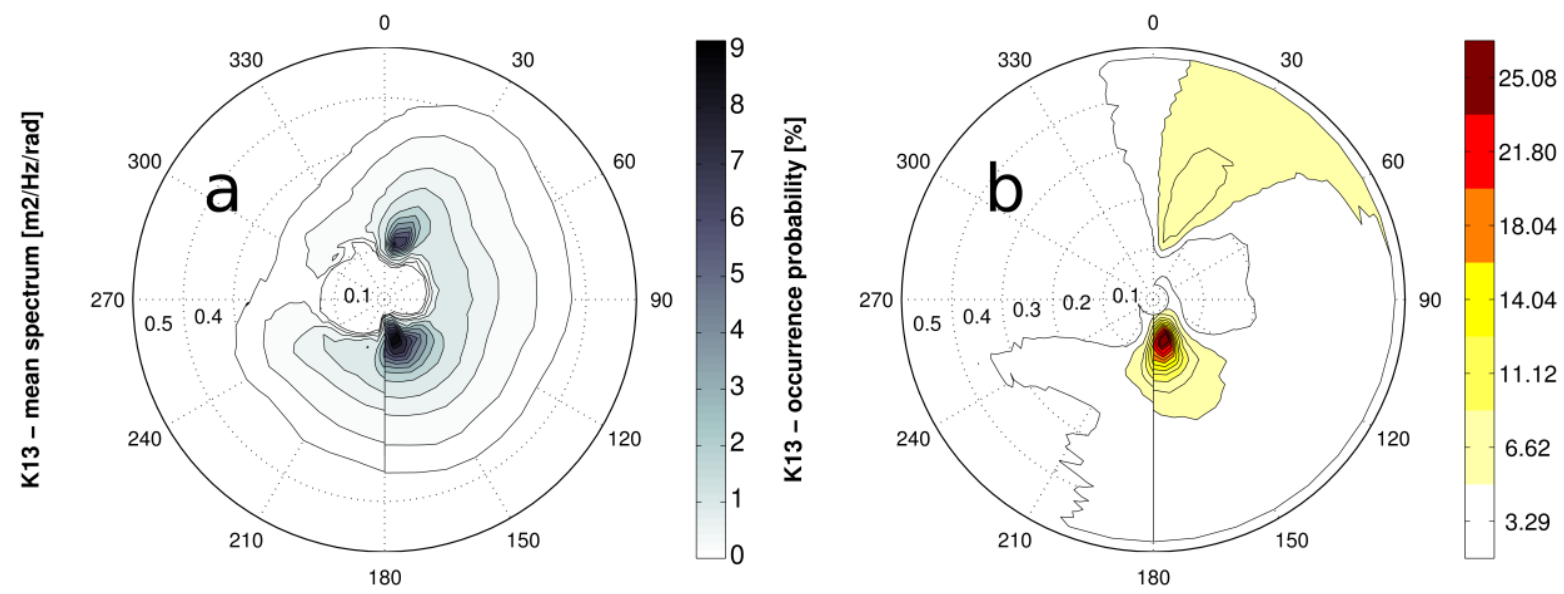

Figure 4.2 - (a) Mean spectrum (from buoy) and (b) occurrence probability (from model data) for the K13 buoy $\left(53.2^{\circ} \mathrm{N}, 3.2^{\circ} \mathrm{E}\right.$; see for reference Figure 4.3). Data are from 2003 to 2007. The radial axis indicates frequency in $\mathrm{Hz}$. Waves are shown in the flow direction (oceanographic convention).

For the present purposes and to illustrate the capability of the method we focus our attention on the area of the Dutch oil platform K13 in the southern North Sea. We use the data from the close-by buoy as supposed source of information for operational application and, at the start, to define the wave climate of the area. The analysis of the locally recorded wave spectra leads to the results shown in Figure 4.2. In panel a we see the local mean spectrum (partitions), dominated by a south going low frequency system (at $\left.180^{\circ}\right)$, and a north-east going system $\left(50^{\circ}\right)$ with a more wind sea appearance. The distinction between the two systems is even more evident in panel b, where (see the previous section) we report the related model derived occurrence probabilities. If the distribution of panel $b$ were known at all grid points in the surrounding area, we could then establish for each point the corresponding cross assignment with the partitions at the K13 position. This would then allow to use the K13 buoy data for DA in operational applications. Obviously we do not have measured data at all the grid points. The solution is to use the output of a locally run wave model as a proxy for the ground truth. Note in this respect that, granted the unavoidable approximations, the model spectra are a more complete and suitable information than the one derived from a buoy (where approximations and sampling variability are always present). For the present example we use a one-year dataset of spectral output from the WaveWatchIII model (Tolman et al., 2013), which is used as a proxy for the ground truth.

The model data come from an ad-hoc implementation in which the spectral output has been saved for every model grid point at three hour interval. The model spatial resolution is 0.1 degrees in latitude and longitude, the spectrum is discretized in 29 frequencies ranging from 0.035 to 0.5 in 1.1 
3877 databases (Smith and Sandwell, 1997). Ice coverage fields and forcing winds are from the NCEP3878 GFS archive with 0.5 degrees resolution. Boundary conditions come from a 0.5 degree global grid 3879 spatial resolution, run together with the North Sea grid using the multigrid capabilities of 3880 WaveWatchIII.

3882 Once a clean signal of the wave systems is obtained on the whole computational domain, we use the scheme given by equations (4.1-4.5) to compute the error correlations. For the two main wave systems of K13, the results are shown in Figure 4.3. It is immediately evident that the two systems have different structures, domains, and magnitudes of the error correlations. The wave system propagating southwards correlates strongly over a larger spatial domain and decorrelates smoothly with distance. Although the errors as such have been calculated on purely statistical bases, physically consistent patterns can be identified.
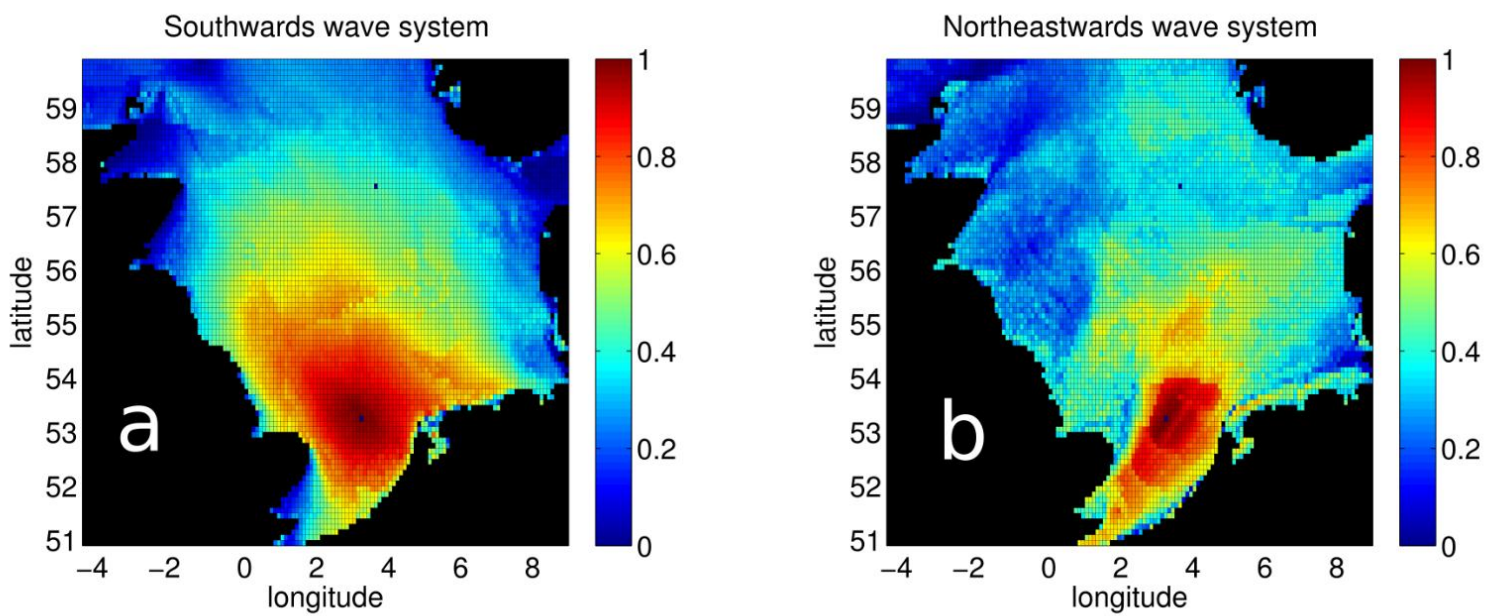

Figure 4.3. Error correlations in the North Sea, for the two main wave systems shown in Figure 4.2, for the assimilation point $\mathrm{K} 13\left(53.2^{\circ} \mathrm{N}, 3.2^{\circ} \mathrm{E}\right)$. a) System flowing southwards, b) system flowing north-eastwards

For instance around the Thames estuary, the correlation reduces sharply because waves from the North are blocked by the East Anglia peninsula. In the southern part around Belgium and the Netherlands, bathymetric effects can be appreciated although bathymetry as such is not explicitly considered in the computations. Its effects appear implicitly from the changes in the spectra, because everything derives from model data.

3900 It is worth stressing that the methodology provides meaningful results also if, to go to the limits, the 3901 wave systems change direction, and possibly period, moving across the grid. This because the cross 
3902 assignment algorithm searches for differentials from point to point. Thus this methodology 3903 overcomes the long standing issue on the complexity of cross-assigning wave systems from 3904 different sources (e.g., Gerlind, 1992, Vorrips et al., 1997, and Corbella et al., 2015).

For the system flowing to North-East, strong correlations are found over a narrow strip, oriented more or less along the coastline. The correlation structure in this case is a lot more irregular and decays slowly along the flow direction, showing large values even in front of the Danish and Norwegian coasts. This is because these wave conditions are more associated to the wind fields (i.e., they are wind waves). The spatial irregularities also suggest that for this less frequent wave system the dataset is too short for the purpose, and that we need longer time series to derive more 3912 robust results. In any case, these two images give a clear view of the error correlation structures 3913 showing that there can be, and are, major differences between the different wave systems. In turn 3914 this indicates that they cannot be assimilated considering the same correlation function.

\section{Lake Michigan case}

3918 Differently from the North Sea, Lake Michigan is a fully enclosed mass of water with characteristic 3919 dimensions 500x200 km. Although the standard wave conditions range from low to mild, during the 3920 autumn months cold northerly and warm southerly fronts may give rise to extreme meteorological 3921 and wave conditions, commonly known as the November gales, which are in turn (the northerly 3922 ones) enhanced by the lake warm waters. Sustained westerly and northerly winds and low-pressure 3923 fronts, accompanied by surge, are also common. In socio-economical terms, the lake hosts many 3924 transportation, commercial, and recreational activities, and hosts large coastal cities like Chicago 3925 and Milwaukee located on the southern shore. For an indication of the wave climate, we use data 3926 from the NDBC buoy 45007 (little circle in Figure 4.5a), and from the ECMWF ERA-Interim 3927 reanalysis (Dee et al., 2011). The results of the analysis for both these sources are presented in 3928 Figure 4.4 where some relevant differences between the buoy and model distributions can be seen. 3929 From the buoy data (panels a, b) we recognize the two cited main wave components, one flowing 3930 northwards, the other southwards. From the mean energy spectrum (panel a) the presence of waves 3931 flowing eastwards can also be appreciated, but these waves do not appear clearly in the probability 3932 density plot (panel b). This suggests that these waves are not so frequent, but experience indicates 3933 that they can be very energetic. On the contrary, in the model distribution these (easterly going 3934 wave conditions) are clearly depicted. As for the two main buoy systems, the southwards one 3935 appears to be flowing mainly at about $200^{\circ}$ in the model (panel d). 
3937 For this example (Figure $4.4 \mathrm{c}$ and $\mathrm{d}$ ) the model data have been taken from the cited ECMWF ERA3938 Interim archive, whose resolution $(111 \mathrm{~km})$, although here interpolated to $0.36^{\circ}$, is much coarser 3939 than desirable for the present purpose, i.e. for an enclosed relatively limited basin. Nevertheless useful results have been obtained. Those for the background error estimates are presented in Figure 4.5. Here panel $a$ shows the bathymetry, the points corresponding to the $0.36^{\circ}$ interpolated ECMWF model grid (in magenta), and the location of the buoy NDBC 45007 (in yellow), assumed as the assimilated observation. Panels $b, c$, and $d$ are the computed correlation structures, with respect to the measurement point, for the three main wave systems flowing northwards, southwards, and eastwards respectively.
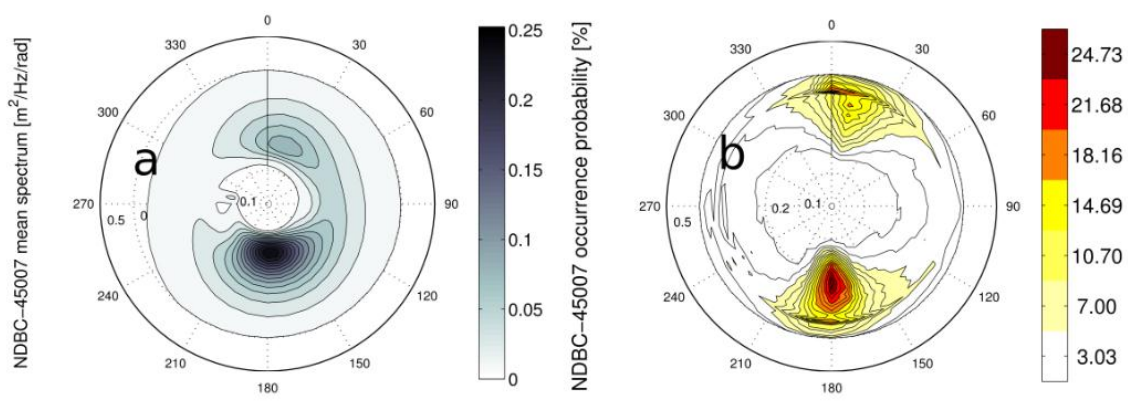

Figure 4.4. a) Mean spectrum and b) occurrence probability for the NDBC 45007 buoy $\left(42.67^{\circ} \mathrm{N}\right.$, 87.0 $\mathrm{W}$; see Figure 4.5 for its position). Buoy data are from 1997 to 2014 . c) mean spectrum and d) occurrence probability for ERA-Interim model data at the buoy location. Model data are from 1979 to 2013. The radial axis indicates frequency from 0 to $0.5 \mathrm{~Hz}$. Waves are shown in the flow direction (oceanographic convention).

3954 Several instructive aspects can be pointed out. In general the three wave systems have similar 3955 structures, the largest one being associated to one of the eastwards systems, with 0.5 values up to 3956 the very north part of the lake. Other physically consistent features can be observed. As expected the largest correlation values in the three cases ( 0.8 and higher) are observed in the region around the buoy, where this is also favoured by the rather homogeneous bathymetry (about 100m). To the 
north of this region, a very shallow feature is present, followed by again deep water conditions (300 $\mathrm{m})$. This discontinuity is clearly reflected into the correlation structure. In the north part the geographical configuration becomes even more complex, including the presence of a mountain 3962 range, and this too affects the wind, hence wave, fields. Another orography derived feature is seen 3963 in $c$, where the correlation values in the easternmost part of the lake are very low because waves 3964 flowing southwards are blocked in the central eastern part of the lake.
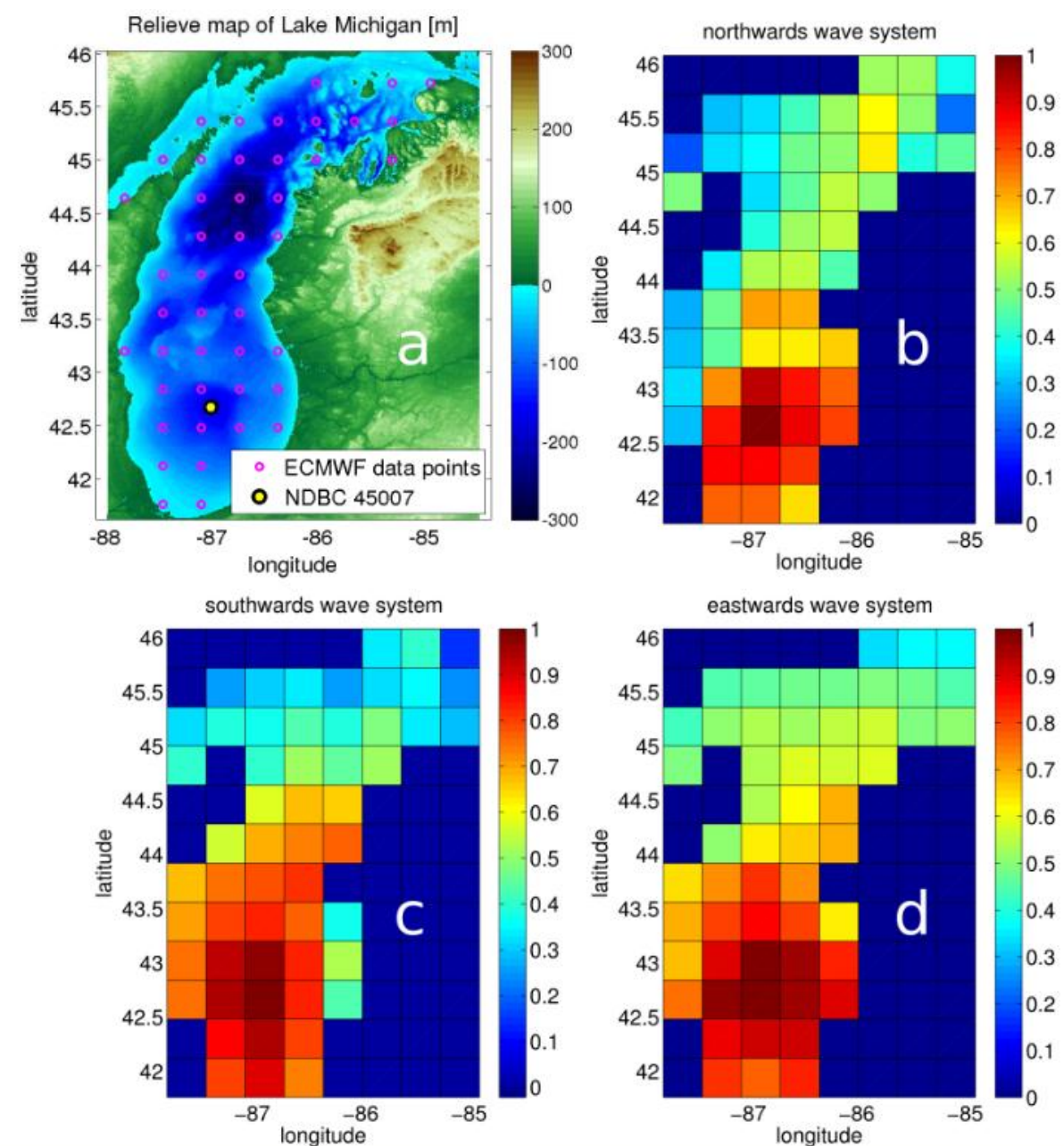

3967 Figure 4.5. a) Lake Michigan depth, position of the reference buoy, and grid point distribution. (b, c, d) Background error correlations for the three main wave systems, seen in Figure 4.4, flowing northwards, southwards, and eastwards respectively. The ECMWF data points in panel a have been interpolated from the original coarser ERA-Interim resolution.

3972 This example has been purposely chosen to give an indication about the spatial resolution 3973 requirements, which for complex configurations, both geographical and meteorological, like the case of Lake Michigan, can be very demanding. This shows how for these situations a much higher resolution is required to carry out the data assimilation with the due efficiency and positive results. 
3976 Note how a preliminary analysis of the background error and its variability in space can be very 3977 useful in planning the geometry of a network of stations to be then used in operational applications.

\section{5 - Discussion, perspectives, and conclusions}

3980 where we summarize our arguments framing the proposed approach within the various systems 3981 presently used

3983 If data are locally available, DA provides the best approach to improve the estimate of the situation 3984 and of the following forecast in complex modelling situations in enclosed seas. This can be done 3985 without breaking the spirit of modern wave models that rely heavily on the physical understanding 3986 of the processes. First, we need to see DA as an extra source term that we must design fully on the 3987 combination of statistical and physical information and of principles. It is clear therefore that for 3988 consistent wave data assimilation, the spectral approach is the way to go. This is not only because 3989 wave spectra as such contain detailed information on the different physical processes, but also 3990 because, failing to consider the wave spectrum, corrections cannot be properly introduced. Most of 3991 the time DA systems based only on integral parameters tend to add noise to the models. Therefore 3992 the spectral DA approach implies also the design of DA system. The result is more consistent with 3993 the model than the present approaches, which are frequently mere adaptations of methods used in 3994 other fields of science as meteorology, where the description of processes and the nature of 3995 variables are completely different.

3997 It is important to note that in the literature, when discussing DA in general, the point often arises of 3998 whether a particular DA scheme (e.g. OI, 3DVAR, 4DVAR) is superior to the other ones. Once we 3999 recognize the fundamental role of background errors in data assimilation, it is clear that this 4000 discussion is immaterial because, if the errors of the system and the spectral update approach have 4001 not been carefully designed, all these schemes would perform poorly. It is certain that the more 4002 requirements we introduce in the cost function, the more constrained the problem is, and therefore 4003 better results should be expected (if we have the proper information). Therefore, a more relevant 4004 question for operational use is how much we can improve the results by adding complexity (and 4005 therefore increasing the computational burden) of the DA scheme. All these difficulties and the 4006 advantage of a sound approach are even more manifest in the difficult environment of the enclosed 4007 seas. 
4009 Two common practices in wave DA implementations are (a) to assimilate integral parameters like $4010 \mathrm{H}_{\mathrm{s}}$, and (b) to parameterize roughly the background error covariance matrix with circular or elliptic 4011 functions decaying exponentially with distance. As shown in the two examples, these practices have 4012 major shortcomings, because the $H_{s}$ value as such does not give any specific information on the 4013 spectral wave systems active at that specific moment on the area we are considering. Besides, as we 4014 have shown, the correlation structures can be rather complex, inherently containing many physical 4015 characteristics of the system, which are not only a simple function of distance. Convenient also in 4016 the oceans, all this becomes mandatory in enclosed seas and coastal areas.

4018 A common and relevant aspect we have mentioned in operational implementations is the 4019 computational cost of DA. Also for schemes like the OI, 3DVAR, and 4DVAR, with the described 4020 approach this is not an issue even if several wave systems are considered because the matrices $\mathbf{B}$ are 4021 precomputed and the optimization step is rather simple. Therefore the cost of these schemes will be 4022 a small fraction of the model computing requirements. That might not be the case for Adjoint or 4023 Kalman filtering schemes for which the costs are of the same order of, or larger than, those of the 4024 model, as on the other hand it is the case also for more conventional approaches. However, the 4025 wave model run as such is relatively cheap, so with the present computing capacities this aspect 4026 should not actually be a barrier. As with any DA system, a more critical requirement in this sense is 4027 the availability of near-real time observations.

4029 Some parallel perspectives of wave DA are first the possible feedbacks that can be established with 4030 the meteorological model. Wave model errors are linked to a large extent to wind field errors, and 4031 this is more the case in the enclosed seas. Hence information about wave model performance 4032 brings, or could bring, immediately information on the driving wind fields. In turn this information 4033 can be useful not only for the wave model, but also to improve surge predictions. Second is the fact 4034 that DA systems, if properly used and interpreted, regularly help to point out deficiencies of the 4035 measured data, and this information can be useful to improve measuring methods and processing 4036 procedures as well. 


\section{L.Cavaleri, A.Sanchez-Arcilla}

luigi.cavaleri@ismar.cnr.it

\section{5 - Summary: the present and the future}

where we summarize the situation of wave modeling in enclosed and coastal seas and try to discuss what the future may hold

Ten years ago a similar paper was issued, discussing the evolving situation in wave modeling, acknowledging the positive results, but also the problems. After fifty years of established background (the energy balance equation applied to wave modeling was made public in 1957), this branch of scientific engineering had reached, via a sometime discontinuous progress, a solid state of maturity. Of course problems were present and expectations were declared. As it is often the case in a mature science (but beware of unexpected steps in knowledge), much action was then put into applications, strongly driven by the social and economic interests in inner seas and close to coasts. In this paper we have focused on these last applications, pointing out the successes and the problems, the well-defined results and the ones we are still aiming to. Driven by both the wish/need for better results and the love for science, somehow our targets keep moving ahead as we progress. Ten years into this new path, it was time to think and define where we are and which our next actions are going to be. It is also instructive to compare the present achievements with the expectations of ten years ago. Although at the time we had a wider perspective (the open oceans) for most of our considerations, unavoidably our present inner and coastal sea focus has ample superposition with our previous interests, and a comparison is possible. In the following we will first frame and discuss the present situation, before venturing into the future trying to guess the developments we expect and would like to achieve in the next 10-20 years.

\section{1 - The present}

where we frame the present situation, highlighting the advancements, but showing also as some of the expectations of ten years ago have not been achieved

It is clear we have done much progress in enclosed seas. Certainly linked to the strongly increased computer power (hence resolution), it is also associated to remarkable improvements in physics of the processes and modelling. In this respect probably the best step ahead has been the substantial level of coupling between waves and current. The better results in both these models allow an effective coupling with strongly improved results, especially in coastal zones. However, these progresses have been partly limited by the strongest level of interaction in the enclosed seas dictated 
4077 by the shorter fetches available, hence by the on average higher frequency waves. Where what 4078 achieved is not yet good enough, we have at least a much clearer idea of the situation. It is 4079 interesting that this development had been fully anticipated ten years ago. Another good guess was 4080 the development and now wide use of unstructured grids to move smoothly from the relatively 4081 coarse resolution offshore to the much more refined one where needed, mostly close to coasts. 4082 Differences of resolution up to three orders of magnitude between the two extremes are common 4083 (but beware of numerics, soon to be discussed).

4084 We had some improvement in physics, but possibly less than expected, at least specifically for 4085 enclosed and coastal seas. In this respect it is the increased resolution that is forcing to consider 4086 some new aspects previously hidden by the coarser approach. Again the main result has been a 4087 much more detailed mutual interaction between waves and currents (the one between waves and 4088 wind was operational since the early '90s). Here part of the credit goes to the much improved 4089 quality of the meteorological input (an amply anticipated development). However, although here 4090 high resolution (order of $1 \mathrm{~km}$ or less) local models are potentially available, still in most of the 4091 cases potentially important details of coastal winds are not up to the level. Sometime we can guess 4092 in qualitative terms what the solution should be, but the model results simply do not provide the 4093 satisfactory quantitative reply we aim to.

4094 Still on the physical side of the problem, there is concern about the modelling of the most intense 4095 storms. It is not the $\mathrm{H}_{\mathrm{s}}$ in itself that is important, but the force with which wind acts on the waves, 4096 especially for a young sea. Citing a recurrent example, the extremely intense blowing of mistral or 4097 bora over a very young short fetch sea leads to conditions that, if witnessed, make one wonder 4098 about the validity of our present approach.

4099 As anticipated ten years ago, there has been a strong development in numerics, and in particular of 4100 unstructured grids. This has been a consequence of the need of high resolution close to coasts, 4101 especially in case of a complicated geometry of the coastline and bathymetry. Indeed, all the 4102 complications of nesting progressively finer grids disappear with the unstructured solution. Of 4103 course this comes at a price: the numerics is more complicated and deserves particular attention. A 4104 strictly "best solution" does not necessarily exist, the most convenient solutionoften being case 4105 specific. The practical implications are not trivial and can lead to substantially different results. The 4106 problem is that in many cases this requires due attention by the user. However, in many cases a 4107 model user is just a user, often taking the model as a black box. While often self-organizing checks 4108 self-control the system, some sensitivity tests would be very useful, especially if the model value is 4109 taken at face value as exact. 
4110 Still within the numericalcomponent, but not limited to unstructured grids, there is still an ample 4111 use of so-called "limiters" in the integration of the model equations. Their reason of being is to 4112 avoid unphysical results of some of the integrated quantities. While properly used they are justified 4113 (a typical non-numerical example is the 0.73 upper limit for the ratio wave height/local depth), we 4114 should never forget that, however justified and well posed, the use of a limiter implies a lack of 4115 physics or proper numerics on our side.

4116 Having listed the successes, it is only fair to see where our expectations were not, or were only 4117 partly, fulfilled.

4118 Bottom processes are still a problem. A good example is given by the just cited (0.73) wave height 4119 limit on a certain depth. Much effort has been recently put to improve this limit or definition, now 4120 forty years old. However, not much improvement has been achieved. This suggests we really do not 4121 have a proper understanding of the related physics. However, for most of the cases the problem is 4122 not, within limits, the lack of physical knowledge. We know which are the basic processes at work, 4123 but we are not completely sure how they interact (e.g., wave and current induced bottom friction) 4124 and, most of all, we often lack the necessary information. This can vary from highly variable 4125 geometry and characteristics to, in case of sandy or muddy bottoms, the potentially quick changes 4126 of the bottom features. For practical purposes the solution, if such, seems to be a sort of ensemble 4127 approach, i.e. different solutions, based on different assumptions, providing the range of possible 4128 outputs.

4129 The correct estimate of nonlinear interactions has not shown drastic advancements, more so in 4130 shallow water. It has been a repeated refrain that "next generation computers will be so fast that we 4131 will be able to use Exact-nl (the related full calculation) in our models". Without exploring the 4132 related quantification, the truth is that, whenever a "ten times faster" computer is available, the 4133 default action is to increase resolution. So we are still with DIA or similar solutions and there is no 4134 perspective of a change of attitude for "next time".

4135 A somehow similar argument, but for different reasons, concerns the fundamentally statistical 4136 spectral approach. There has been much debate wondering if it makes sense, as we do with spectral 4137 models, to go to spatial resolution shorter than the wavelengths we want to describe. Of course in 4138 this case our estimated spectrum has a correspondence only in time, not in space. However, there 4139 had been much talking about, e.g. spectra and bispectra, to provide more physical results. While 4140 studies continue, for routine practical applications we are still on the traditional ground.

4141 In enclosed and coastal seas, we face a problem with data assimilation. It is clear that the limited 4142 dimensions we deal with imply a shorter memory in the system. Therefore, most of the time the 4143 focus is on very short forecast ranges. This concerns the distributed information, i.e. satellite data. 
4144 Buoys, mostly close to coast, have a more limited time scope, being mostly used either for 4145 verification of the incoming conditions or for nowcast with a horizon of one hour or so. Use in the 4146 opposite direction, i.e. for offshore blowing wind, is limited because of the relatively poor 4147 correlation between short fetch conditions, especially the modelled ones, and the more developed 4148 ones in the rest of the basin.

4149 A very positive aspect with respect to ten years ago is the convergence of the models towards a 4150 more unified and agreed upon physics. It is a recurring process. Of the various branches offering 4151 different approaches, in time one emerges as the best and most satisfactory, where everyone 4152 converges. But then we go into deeper details, with different approaches, and from the "winning" 4153 branch smaller branches sprout associated to different solutions, and the cycle repeats. This is a 4154 healthy process, exploring different grounds. This is the present situation, with substantially similar, 4155 but slightly different, physics (possibly coefficients), with a continuous mutual exchange of 4156 information and cross-comparison of results. This is a very satisfactory situation, forerunning 4157 further advancements.

4158 We have devoted a strong effort to make clear to the user that the data we use to improve or validate 4159 our models are just estimates of the truth. Pretty good estimates in most of the cases, but with well4160 defined confidence limits. On one hand these derive from the random variability of the wavy 4161 process. To be practical, the significant wave height derived from a 20 minute buoy record has an 4162 uncertainty of $6 \%$ at $90 \%$ confidence. On the other hand, an instrument provides the local truth only 4163 with errors, and these errors can be quite significant. This should be taken into account when 4164 judging the performance of a model. Incidentally this is one of the reasons while in practical terms 4165 the model root mean square error cannot be lower than a certain value.

4166 Somehow this takes us to the last topic of this picture of the present. Most of the validations we do 4167 with our model results are based on integral parameters, typically $H_{s}, T_{m}$ or $T_{p}, \theta_{m}$ (with their 4168 confidence limits). However, it is amply acknowledged that, although direct and intuitive, most of 4169 the time these parameters do not provide by themselves a satisfactory view of the situation. Clearly 4170 the best information is provided by the 2D spectrum. This has two drawbacks: a) the spectrum is not 4171 always available, b) if it is, it has very large (much more than the $6 \%$ for $\mathrm{H}_{\mathrm{s}}$ ) confidence limits, a 4172 hardly mentioned inconvenient truth. An intermediate solution is provided by the partition 4173 approach, i.e. by identifying in the 2D spectrum the different wave systems that compose it, and 4174 providing the integral parameters for each composing system. This will also be the background for 4175 the data assimilation that that we discuss in "The future" section. 
where we list the areas where developments and a more intense use of present facilities are expected and needed, and where, although presently not sufficiently considered, we need to put attention in the future

Our look into the future needs to be not only an extrapolation of, e.g., the last ten years. This would be, so to say, a passive attitude. Rather, our analysis of the present situation must also suggest where, following our perceptions of the future needs, we should drive, if however possible, the system in the future, all this within the limits of human attitude and interests.

By and large the basic physics of wave models is considered well established (but see comments below), and it would be natural to think that improvements in the near future will be quite marginal. There are some 'however'. First, we still have the problem of white-capping. Modellers may appreciate the availability of a "tuning knob" to drive the model to the best (on average) results. It is interesting that, if we had purely physically specified source functions and the results were not good, in principle we would not know where to act.

On the other hand the dramatic improvements in highly detailed computer simulations of the air-sea interaction processes hint to substantial advances in the understanding and quantification of the involved energy exchanges. Besides the great progresses in experimental and measured characteristics of, and energy involved in, white-capping with the consequent bubble plumes and spray suggest a possible future shift of some of the pressure for better knowledge on the wind input side, for long assumed a strong stronghold of the overall theoretical approach.

Still at a general level, and again not limited to enclosed and coastal seas, there are the attempts to solve the energy balance equation via a full evaluation of the nonlinear interactions with all the implications for the other source terms. While this does not seem to be a realizable target in the near future, it is something that, if happening, would shake heavily the whole system.

At a more fundamental level we should consider the hypotheses that are at the base of our wave energy or action balance equation and wonder if and how we need to modify it to go further. On a broad scale we can question, and explore the limits of, the validity of the linear wave theory at the base of our spectral approach (the fourth-order nonlinear wave-wave interactions are not the full solution). The first and relatively simplest correction can be for inhomogeneous media and to make the advection amplitude dependent. This is obviously also connected to what we call bound modes. Again questioning the base of the wave action equation we need to realize that this equation is a good approximation to the truth only in deep water. Moving to shallow areas nonlinearities become more important, in reality more than most of us think. There is a diffused belief that, also when accordingly modified, the present formulation does not satisfy the needs in shallow water. 
4212 Approaching this area the importance of triads progressively emerges. However, considered in 4213 SWAN, their present formulation is only a crude approximation to the truth. In our continuous quest 4214 for better results, this will have to be taken into consideration and given a workable solution.

4215 Another fundamental advancement, on which work has been done for a number of years, concerns a 4216 generalized evolution equation for the transport of the coupled second order wave statistics of the 4217 surface elevation. If successful, this will bring the field beyond the present idea of energy spectrum. 4218 However, this approach is still at the experimental level and the practical implications for the real 4219 daily activities are not yet clear.

4220 A process till now considered mainly from the theoretical point of view is Bragg resonance with the 4221 bottom spectrum. Apart from the involved computations, the point is that we mostly lack the 4222 necessary information (the 2D spectrum, in principle also time dependent). However, in some cases 4223 this process will have to be considered because its potential influence on the final results can be 4224 very large.

4225 A further physical frontier we will need to deal with is a more detailed description of what is going 4226 on in the breakers zone on the shore. There is a tremendous amount of energy dissipated here in 4227 very short space and time. More importantly, this area is crucial for several relevant processes 4228 whose present solutions are not fully satisfactory. Physical and numerical advancements are here 4229 required.

4230 Discussing "The present" situation we have mentioned that most of the physics in our wave models 4231 loses its validity under extreme conditions. Indeed, it is amazing, and it should be a valuable piece 4232 of information, that, notwithstanding such limitation, we get reasonably good results. In any case 4233 white-capping, together with its interaction with wind, leads to spray that is considered as the most 4234 effective transfer of matter and heat to the atmosphere. This process is connected to the generation 4235 by wind. As a matter of fact, we are here at a choice: either we accept that in these strongly forced 4236 conditions the physics is different, or, if we consider our physics universally valid and we know the 4237 conditions are different, we must acknowledge that something is not fully correct in our present 4238 view of the generation/dissipation processes. It is worth to specify that these, if not extreme, highly 4239 pushed conditions, are possible and relatively frequent also in enclosed seas. We recall the cited 12 4240 m significant wave height in the "small" Adriatic Sea and, for a different situation, the highly 4241 generative limited fetch conditions present in the Gulf of Lion and the Northern Adriatic Sea, 4242 respectively under mistral and bora wind.

4243 Under what we can call "normal" conditions the physics of wave-current interactions is well 4244 developed, but some aspects still need to be improved. The question often is if the full necessary 4245 information is available. Especially close to coast, with a pronounced orography, the role of the 
wind field may be crucial, affecting both the local waves and current, with mutual interactions. In these highly variable conditions we frequently lack one of the implicit assumptions, i.e. that the current is vertically uniform, at least within the wave vertical range. Present wave models are not prepared for a vertically non-uniform current. More in general the overall view of the wave-current interactions will have to move towards a more 3D approach, and this is more true in coastal waters where both the fields, waves and current, are modified by the limited vertical scope.

This more intense interaction rises the problem of the calibration of the models we use. The expression for the various processes have by default been obtained in 'normal' conditions, i.e. in general without much influence by currents. It is meaningful to wonder if these calibrations still hold when there is a strong 3D interaction with current. This is an open problem that does not have an immediate solution.

The need for a deeper look to currents is also connected to their stratification and interaction with the atmosphere. A general problem of circulation modelling is the frequently too shallow thermocline. Waves have their role in this respect, as also has the Langmuir circulation whose generation requires the coupling of the full meteo-ocean system. More in general, as wave modellers acting frequently in coastal water conditions, to consider the interaction with currents is practically a "must". The problem is that circulation modellers still drive their currents with the wind stress, ignoring that most of the related energy and momentum transfers happen via wind waves. In the long term we will have to get used to talk about the wave-current system as a single, possibly also with wind, logical unit. This will be, and already is, crucial when dealing with coastal flooding under strong stormy conditions. What shown for Katrina and the Gulf of Mexico coast is a very convincing argument.

Computers will become faster and more powerful. This will push users toward higher resolutions. It is an interesting question why we like to think that, or act as, a higher resolution will always be "the solution". It is true that, as mentioned above, we often lack detailed information (a highly variable bathymetry, or think of the fractal conditions at the southern border of the Bothnian Sea), but 1) if we go to higher resolution, a meaningful job requires a correspondingly detailed information, 2) we must be sure that what we model is the truth, 3) there is the non-trivial problem of natural variability. When modeling an environment with a relatively coarse resolution, we implicitly produce results that represent, right or wrong, the average conditions at each mesh point (in space and time). However, as continuous high resolutions show, or as, within limits, seen in scatterometer and SAR images, nature performs with a high (conditions dependent) natural variability. If the physics of our model progresses with its resolution, this variability must and will appear also in the model results. However, we should never forget that, the more we go into resolution, the more our 
results, although partially deterministic in space, will be always only statistically valid in time. For obvious reasons we do not have the corresponding, in space and time, measured information. Note that this may be true also for full basins or full ocean (or global) meteorological models if pushed beyond a certain resolution. This does not mean this variability will be useless. Possibly fundamental for the local physics (e.g., integral fluxes), we will use it in statistical terms to achieve further knowledge in relatively high frequency processes, e.g. the characteristics of gustiness. Note that, within limits, this will be less the case for wave results because of their characteristics of being an integral in space and time of the driving wind field. Their variability may not be trivial for special cases, e.g. short fetch generation of very gusty winds (bora and mistral).

The high resolution leads us to talk about numerical grids. There is no doubt that unstructured grids will be the solution for improving the resolution where required in enclosed and coastal seas. On a wider scale, for a number of years there has been some interest in the capability of dynamically adapting the local conditions of the grid where required by, e.g., the stronger spatial gradients. A common problem reported from a similar approach in circulation modelling is the continuous interpolation required and the consequent progressive smoothing of the field. This may well be the case, however, on one hand the results will certainly be better than with a coarse (relatively speaking) resolution, on the other one new grid adaptations are not required at each time step and can be partly coincident with the previous one, in so doing strongly reducing the problem. Indeed this seems to be the only approach to a proper dealing of cases like hurricanes where, whichever the resolution of the global or, e.g., Atlantic, model, it will not be sufficient for a proper description of their central part. Note that purposely we do not touch here the corresponding meteorological problem.

Still related to grids, and pushed by the use of unstructured ones, numerics has become in our field a very dynamical subject where advancements and improvements are to be expected. We are presently well aware of the problems we are facing, the key point being that with the present numerical methods we cannot satisfy all the requirements of an ideal solution, both as computer and accuracy (not to mention the numerical details). New methods need to be developed, and this will be more and more the case as we move on one hand to more extreme conditions (this is possible also in the enclosed seas), on the other hand to more and more shallow water with higher and higher resolution. The link with the physics in the breakers zone is obvious. As with physics, there is a level of conservatism in our present approaches and solutions. The person considering physics or numerics as only tools to reach the real target, e.g. the maximum wave height in front of a harbour, may be happy to have the computer machine running smoothly. However, the specialist of the subject, in our case the numerical expert, may point out a number of potential, perhaps hidden, 
4314 problems and push for a new solution. From a more general perspective, the existence of a 4315 discussion, not only of alternative solutions, suggests that the field is not yet sufficiently mature. 4316 Indeed, in other fields of science, including mathematics, there are more profound studies on the 4317 integration of partial differential equations. We should always be open to these new solutions, 4318 especially when their correct use could appreciably, if not more, change our results. This is a very 4319 general problem, i.e. the balance between specialized and focused knowledge, where, zooming 4320 more and more on a specific subject, we tend to ignore the useful progresses in parallel sciences. 4321 The interesting experiment of representing the spatial distribution of the wave fields in spectral 4322 mode (as in spectral meteorological models) is instructive in this respect.

4323 Discussing numerics involves the use of limiters. Of course at present they are needed and useful. 4324 However, as just said, the fact that our machine is running smoothly and meaningful results pop out 4325 does not imply that what we are doing is correct. Every limiter implies something is wrong in our 4326 approach. In a way they are useful because they tell us where we need to act, let this be physics or 4327 numerics.

4328 A final subject bordering between physics and numeric is the use of phase resolving models. The 4329 dream of a fully deterministic approach (assuming the corresponding input information is available) 4330 has lured engineers for decades. Without discussing the possible spatial extent of these simulations 4331 before the spatial resolution takes its toll, the present and for the foreseeable future drastic limit to 4332 their use is still the required computer time.

4333 In "The present" we have mentioned what we can call the limitations of data assimilation in 4334 enclosed and coastal seas, i.e. its limited validity in time: the information, i.e. the waves, will 4335 rapidly reach the coast. With respect to the open ocean there is also a more pronounced asymmetry 4336 in the fields. This implies that the usual empirical default distributions of the range of influence of a 4337 give piece of information may not necessarily lead to a good job. Indeed, as we discussed in 4338 Chapter 4, a preliminary study is required for any area of interest, providing, for every point of the 4339 grid, hence for every measured datum available and for a given wave system, the corresponding 4340 map of influence. We believe this is the method that, valid also on the open ocean, will be more so 4341 in enclosed seas.

4342 Much more attention needs to be paid to the errors of measured data. While we expect an improved 4343 quality in some aspects or types of instruments, there is a strong possibility that the one of the 4344 master reference in our field, i.e. buoys, is deteriorating because of the need to decrease their price 4345 and handling costs. A strong push should be done on management and manufacturers to make 4346 available, better if in real time, the raw original data. This would allow improved analyses of 4347 directional distributions, single, including freak, wave heights, and, where done, intercomparisons 
4348 of different instruments. Campaigns in this last direction are strongly recommended. In any case 4349 comparison of model data versus measured ones must take the measurement errors into 4350 consideration. An open problem is that these errors are frequently not available.

4351 Much expectation exists for satellite data close to coast. Indeed, the Sentinel 3 approach of slicing 4352 the explored area in $300 \mathrm{~m}$ long (in the direction of flight) and a few kilometers wide zones sounds 4353 very promising, especially if the flight direction is perpendicular to the coastline. However, for the 4354 time being and the immediate future the errors, instrumental and statistical, present in those data can 4355 be accepted using the data for some purposes, but their use for model validation remains at least 4356 debatable. Progresses are expected, but far from the accuracy of a local measurement.

4357 Some of the refinements in the physics of wave models, especially in wave-current interactions or 4358 in some aspects of non-linearity (e.g., bound modes), badly need more information than the 4359 classical measurements. To a large extent, either shot views of a field or time measurements at a 4360 single point, all rely on or assume linear theory. However, in a way this is one of our present limits. 4361 Measurements as the cited stereo ones, providing both $\mathrm{k}$ and $\mathrm{f}$ directional spectra, are badly needed 4362 to progress further on a solid ground. Luckily this technology is now available and well described. 4363 Granted the availability of platforms and rigs, this technology is expected to have a large diffusion.

4364 The uncertainty that characterizes every estimate, either as hindcast or forecast, and the need to 4365 provide also this information to the user will lead more and more toward the use of ensemble 4366 approaches. Already present at some of the forecasts centres, we expect their use will increase in the 4367 future, especially where and when the specific value of one or more parameters is of concern. The 4368 problem is that at present the ensemble is (mostly) only meteorologically driven, while the wave 4369 model (and current one as well), is (are) taken as deterministically correct. However, we know this 4370 is not true. We can make a model ensemble, but this is too limited. As in meteorological modelling, 4371 new tools need to be developed to have ensemble wave runs. Of course, at centres such as ECMWF 4372 the wave model has been for a long while two-way coupled to the meteorological one, so that the 4373 suggested flow seems natural. However, again this corresponds to taking into account the 4374 meteorological uncertainty, while the one in the wave model also needs to be considered.

4375 The thin line between rigour and pragmatism concerns the approach to a specific problem, e.g. the 4376 forecast of the wave conditions at a certain harbour. It is clear that in general we pursue the solution 4377 of the general problem, i.e. wave conditions in enclosed seas. However, given a certain target, 4378 possibly limited in space, there will always be a greater level of empiricism if experience shows that 4379 a certain pragmatic solution provides the best results.

4380 On a more general and final perspective, our aims and expectations of wave model improvements, 4381 however important, are not the whole purpose. It is clear that in general we are moving toward a 
4382 fully coupled atmosphere-ocean system where the waves are the modulating interface. Indeed, both 4383 if we consider a developing cyclogenesis or work as far as climate models (but most of them ignore 4384 waves), waves are the key knob that modulates all the heat, matter, energy et al. transfers at the sea 4385 surface. This is another enormous field still in its initial stage, but that badly needs devoted activity 4386 and drastic developments. It is interesting, instructive and stimulating how, in solving a problem, 4387 we go from the focused attention of a technical detail to the ample view of the Earth system.

\section{Acknowledgements}

4393 José-Henrique Alves, Sabique Langodan, Roberto Padilla-Hernandez, Kirti Samiksha Volvaiker 4394 contributed and provided material for this paper. They appear as co-authors in some of the Sections. 4395 Then years ago a similar white-paper on wave modelling was published as a product of the WISE 4396 Group. In this period the Group has grown and in practice it is no longer possible to produce now a 4397 Group-paper. However, all of us who have actually worked on it must and are pleased to 4398 acknowledge that, at least in spirit, this is a community product, derived from much work from 4399 most of the authors, but also from the long term interactions and sometime hot discussions that are 4400 at the base of a community science where honesty, friendship and a love for nature are the keys to 4401 success. However, both for the benefit of us and the ones who will follow it is necessary to frame 4402 every now and then the situation. Using an oftern used parallelism, it is like climbing a mountain. 4403 Every so often we need to put a pin in the rock of the cliff are are ascending to know better, and to 4404 be sure of, where we are and proceed further on solid ground. 


\section{References}

Abdalla, S., and L.Cavaleri, 2002, Effect of wind variability and variable air density on wave modelling, J.Geoph.Res., 107, No.C7, 17-1/17-17.

Abdalla, S., and P.A.E.M.Janssen, 2017. Monitoring waves and surface winds by satellite altimetry: applications, in Satellite Altimetry over Oceans and Land Surfaces, D.Stammer and A.Cazenave (eds.), CRC Press, Taylor \& Francis Group, USA, 379-424.

Abdalla, Saleh, Janssen, Peter A. E. M. and Bidlot, Jean-Raymond (2010): "Jason-2 OGDR Wind and Wave Products: Monitoring, Validation and Assimilation", Marine Geodesy, 33: 1, 239-255. DOI: $10.1080 / 01490419.2010 .48779$

ACT - Alliance for Coastal Technologies, 2007. ACT Wave Sensor Technologies. Proceedings of a Workshop held by the Alliance for Coastal Technologies, March 7-9 2007, St. Petersburg, FL, 26pp, http://www.act-us.info/Download/Workshops/2007/USF_NDBC_Wave/ .

ACT - Alliance for Coastal Technologies, 2012. Wave measurement systems test and evaluation protocols in support of the National Operational Wave Observation Plan, Proceedings of a Workshop held by the Alliance for Coastal Technologies, February 22-24 2011, St. Petersburg, FL, 29pp, http://www.act-us.info/Download/Workshops/2012/USFUM_Wave_Measurement/ .

Adam A, Buchan AG, Piggott MD, Pain CC, Hill J, Goffin MA, 2016, Adaptive Haar wavelets for the angular discretisation of spectral wave models. Journal of Computational Physics, 305, 521-538. doi:10.1016/j.jcp.2015.10.046

Alari, V, J Staneva, O Breivik, J-R Bidlot, K Mogensen, P Janssen (2016). Surface wave effects on water temperature in the Baltic Sea: simulations with the coupled NEMO-WAM model, Ocean Dyn, 66, pp 917-930, doi:10.1007/s10236-016-0963-х

Alfieri L., Salamon P., Pappenberger F., Wetterhall F., Thielen J., 2012, Operational early warning systems for water-related hazards in Europe, Environmental Science \& Policy, 21, 35-49, ISSN 1462-9011, http://dx.doi.org/10.1016/j.envsci.2012.01.008.

Allender, J., Audunson, T., Barstow, S.F., Bjerken, S., Krogstad, H.E., Steinbakke, P., Vartdal, L., Borgman L.E., Graham, C., 1989. The WADIC project: A comprehensive field evaluation of directional wave instrumentation. Ocean Engng, Vol. 16 No 5/6, 505-536.

Alves J.H., Chawla A., Tolman H., Schwab D., Lang G., Mann G., 2014: The Operational Implementation of a Great Lakes Wave Forecasting System at NOAA/NCEP. Wea. Forecasting, 29, 1473-1497. doi:10.1175/WAF-D-12-00049.1

Anderson, M.E., Smith, J.M., 2014. Wave attenuation by flexible, idealized salt marsh vegetation. Coastal Engineering, 83, 82-92.

Appel, K.W., A.J. Riordan, and T.A. Holley, (2005). An objective climatology of Carolina coastal fronts, Wea. Forecasting, 20, 439-455. 
Ardhuin, F. and A. D. Jenkins, 2006: On the interaction of surface wave and upper ocean turbulence. J. Phys. Oceanogr., 36, 551-557.

Ardhuin, F., A. Roland, F. Dumas, A.C. Bennis, A. Sentchev, P. Forget, J. Wolf, F. Girard, P. Osuna, and M. Benoit. 2012. Numerical Wave Modeling in Conditions with Strong Currents: Dissipation, Refraction, and Relative Wind. J. Phys. Oceanogr., 42(12), 2101-2120.

Ardhuin, F., and A.D. Jenkins. 2005. On the interaction of surface waves and upper ocean turbulence. J. Phys. Oceanogr., 36, 551-557.

Ardhuin, F., Collard, F., Chapron, B., Ardhuin, F.-G., Guitton, G., Mouche, A., Stopa, J.E., 2015. Estimates of ocean wave heights and attenuation in sea ice using the SAR wave mode on Sentinel1A, Geophys. Res. Lett., 42, 2317-2325, doi:10.1002/2014GL062940.

Ardhuin, F., Dumas, F., Bennis, A.-C., Roland, A., Sentchev, A., Forget, P., Wolf, J., Girard, F., Osuna, P., Benoit, M., 2012. Numerical wave modeling in conditions with strong currents: dissipation, refraction and relative wind, J. Phys. Oceanogr., 42, 2101-2120.

Ardhuin, F., Herbers, T.H.C., 2002. Bragg scattering of random surface gravity waves by irregular sea bed topography. J. Fluid Mech., 451, 1-33. DOI: 10.1017/S0022112001006218

Ardhuin, F., Herbers, T.H.C., van Vledder, G. Ph., Watts, K.P., Jensen, R., Graber H.C., 2007. Swell and slanting-fetch effects on wind wave growth, J. Phys. Oceanogr., 37(4), 908-931.

Ardhuin, F., N. Rascle, and K. B. Belibassakis, 2008: Explicit wave-averaged primitive equations using a generalized lagrangian mean. Ocean Modell, 20, 35-60.

Ardhuin, F., Rogers, W.E., Babanin, A.V., Filipot, J.-F., Magne, R., Roland, A., Van der Westhuysen, A.J., Queffeulou, P., Lefevre, J.M., Aouf, L., Collard, F., 2010. Semi-empirical dissipation source functions for ocean waves. Part I: definition, calibration, and validation. J. Phys. Oceanogr. 40, 1917-1941.

Ardhuin, F., Roland, A., 2013. The development of spectral wave model: coastal and coupled models. Coastal Dynamics, Arcachon, France.

ASCE, Hamburg, Germany, vol. 1, pp. 569-587

Babanin, A. V. (2006), On a wave-induced turbulence and a wave-mixed upper ocean layer, Geophys. Res. Lett.,33, L20605, doi:10.1029/2006GL027308.

Babanin, A. V. and B. K. Haus, 2009: On the existence of water turbulence induced by nonbreaking surface waves. J. Phys. Oceanogr., 39, 2675-2679.

Babanin, A. V., A. Ganopolski, and W. R. Phillips (2009), Wave-induced upper-ocean mixing in a climate model of intermediate complexity, Ocean Modell., 29(3), 189-197, doi:10.1016/j.ocemod.2009.04.003

Babanin, A.V, Makin, V.K., 2008. Effects of wind trend and gustiness on the sea drag: Lake George study. J. Geophys. Res. 113, C0215: doi:10.1029/2007JC004233.

Babanin, A.V., T.-W.Hsu, A. Roland, S.-H.Ou, D.-J.Doong, and Y.-M. Fan, 2010: Spectral 
modelling of Typhoon Krosa. Natural Hazards and Earth System Scie.

Bakhoday-Paskyabi, M., I. Fer, and A. D. Jenkins, 2012: Surface gravity wave effects on the upper ocean boundary layer: Modification of a one-dimensional vertical mixing model. Cont. Shelf Res., $38,63-78$.

Balmaseda, M A, K Mogensen, A T Weaver (2013). Evaluation of the ECMWF ocean reanalysis system ORAS4, Q J R Meteorol Soc,139 (674), pp 1132-1161, doi:10.1002/qj.2063

Barthelmie, R.J., (1999). The effects of atmospheric stability on coast wind climates, Meteorol. Appl. 6, 39-47.

Barthelmie, R.J., B. Grisogono, and S.C. Pryor, (1996). Observation and simulations of diurnal cycles of near-surface wind speeds over land and sea, J. Geophy. Res, 101, No. D16, 21327-21337.

Battjes, J.A., 1994. Shallow water wave modelling, Proc. Int. Symp: Wave - Physical and numerical modelling. University of British Colombia, Vancouver, Canada.

Battjes, J.A., Janssen, J.P.F.M., 1978. Energy loss and set-up due to breaking of random waves. In: Proc. 16th Int. Conf. Coastal Eng., ASCE, Hamburg, pp. 569- 587.

Beardsley R. C., C. Chen, Q. Xu., 2013. Coastal flooding in Scituate (MA): A FVCOM study of the 27 December 2010 nor'easter. JGR Oceans, 118, 6030-6045, doi:10.1002/2013JC008862

Becq-Girard, F., Forget, P., Benoit, M., 1999. Non-linear propagation of unidirectional wave fields over varying topography, Coastal Eng. 38, 91-113.

Behrens, A., 2015: Development of an ensemble prediction system for ocean surface waves in a coastal area. Ocean Dynamics, Volume 63, Issue 4 (2015), pp 469-486

Bender, L. C., Guinasso, N.L., Walpert, J.N., 2010. A comparison of methods for determining significant wave heights-Applied to a 3-m discus buoy during Hurricane Katrina, J. Atmos. Oceanic Technol., 27, 1012-1028.

Bender, L.C., Guinasso, N.L., Walpert, J.N., Howen, S.D., 2009. A comparison of two methods for determining wave heights form a discus buoy with a strapped-down accelerometer, Proceedings 11th International Workshop on Wave Hindcasting and Forecasting, Halifax, Canada.

Benetazzo A., A. Bergamasco, D. Donaldo, F.M. Falcieri, M. Sclavo, L. Langone, and S. Carniel. 2014. Response of the Adriatic Sea to an intense cold air outbreak: dense water dynamics and waveinduced transport. Prog. Oceanogr., 128, 115-138.

Benetazzo A., Barbariol F., Bergamasco F., Torsello A., Carniel S., Sclavo M., 2015. Observation of extreme sea waves in a space-time ensemble. J. Phys. Oceanogr., 45, 2261-2275. doi:10.1175/JPOD-15-0017.1.

Benetazzo A., S. Carniel, M. Sclavo and A. Bergamasco. 2013. Wave-current interaction: effect on the wave field in a semi-enclosed basin. Ocean Model., 70, 152-165.

http://dx.doi.org/10.1016/j.ocemod.2012.12.009.

Benetazzo, A., F. Fedele, G. Gallego, P.-C. Shih, and A.Yezzi, 2012. Offshore stereo measurements 
of gravity waves, Coast.Eng., 64, 127-138.

Benetazzo, A., F.Barbariol, F.Bergamasco, A.Torsello, S.Carniel, and M.Sclavo, 2015. Observation of extreme sea waves in a space-time ensemble, J. Phys. Oceanogr., 45, 2261-2275.

Benoit, M., 1992. Practical comparative performance survey of methods used for estimating directional wave spectra from heave-pitch-roll data, Proceedings of the 23rd International Conference on Coastal Engineering, ASCE, 62-75.

Benoit, M., Marcos, F. and Becq, F., 1996. Development of a third generation shallow-water wave model with unstructured spatial meshing. Proc. 25th Int. Conf. on Coastal Engineering, ASCE, 465478.

Bertin, X., Li, K., Roland, A., Bidlot, J-R., 2015 Contribution of short-waves in storm surges: two case studies in the Bay of Biscay, Continental Shelf Research, Elsevier, 2015,

$<10.1016 /$ j.csr.2015.01.005

Bertotti L., Cavaleri L., Soret A., Tolosana-Delgado R., (2014), Performance of global and regional nested meteorological models, Continental Shelf Research, 87, 17-27, ISSN 0278-4343, http://dx.doi.org/10.1016/j.csr.2013.12.013.

Bertotti, L., L.Cavaleri, L.Loffredo, and L.Torrisi, 2013, Nettuno: analysis of a wind and wave forecast system in the Mediterranean Sea, Monthly Weather Review, 141, No.9, 3130-3141, doi:10.1175/MWR-D-12-00361.1

Bertotti, L., and L. Cavaleri. 1985. Coastal set-up and wave breaking, Oceanologica Acta, 8, N.2, 237-242.

Bertotti. L., P.Canestrelli, L.Cavaleri, F.Pastore, and L.Zampato, 2011. The Henetus wave forecast system in the Adriatic Sea, Nat. Hazards and Earth Syst. Sci., 11, 2965-2979.

Bidlot, J.-R., 2017. Twenty-one years of wave forecast verification, ECMWF Newsletter, No. 150, 29-32, DOI: 10.18442/ECMWF-NL-201610

Bidlot, J.-R., Hansen, B., Janssen, P.A.E.M. , 1997. Wave modelling and operational forecasting at ECMWF, in: Stel, J.H. et al. (Ed.) (1997). Operational oceanography: the challenge for European cooperation: Proceedings of the First International Conference on EuroGOOS 7-11 October 1996, The Hague, the Netherlands. Elsevier Oceanography Series, 62: pp. 206-213.

Bidlot, J-R (2012). Present status of wave forecasting at ECMWF in Workshop on Ocean Waves, 2527 June 2012. Available online at http://www.ecmwf.int/publications/, 15 pp

Birkemeier, W.A. and E.B. Thornton, (1994). The DUCK94 Nearshore Field Experiment, Proc. Conference on Coastal Dynamics '94, 815-821.

Birkemeier, W.A., Jensen, R.E., Bernard L.J., Bouchard, R., 2012. OOOS wave observations, a national perspective, MTS/IEEE Oceans 2012, 14-19 October 2012, Hampton Roads, VA, DOI:10.1109/OCEANS.2012.6405055 .

Bishop C.T., Donelan, M.A., 1987. Measuring waves with pressure transducers, Coastal Engineering 11, 309-328. 
Björkvist, J.-V., Tuomi, L., Fortelius, C., Pettersson, H., Tikka, K., Kahma, K., 2016. Improved estimates of near shore wave conditions in the Gulf of Finland. Journal of Marine Systems, doi: 10.1016/j.jmarsys.2016.07.005.

Blain, C.A., R.S. Linzell, P. Chu, and C. Massey. 2010. Validation Test Report for the Advanced CIRCulation Model (ADCIRC) v45.11. NRL/MR/7320-10-9205, Naval Research Lab, Stennis Space Center, MS, 109pp.

Blumberg, A F and G L Mellor (1987). A description of a three-dimensional coastal ocean circulation model in Three-Dimensional Coastal Ocean Models, N S Heaps (ed), AGU Coastal and Estuarine Series 4, American Geophysical Union, Washington D C

Bolaños, R., P.Osuna, J. Wolf, and A. Sanchez-Arcilla. 2011. Development of the POLCOMS-WAM current-wave model. Ocean Model., 36, 102-115.

Booij, N. and Holthuijsen L.H., 1987Propagation of coean waves in discrete spectral wave models. J. Comput. Physic, 68, 2, 307-326, DOI: 10.1029/98JC02622

Booij, N., R.C. Ris, and L.H. Holthuijsen. 1999. A third-generation wave model for coastal regions, Part I, Model description and validation. J. Geophys. Res.-Oceans, 104, 7649-7666.

Bosart, L.F., (1975). New England coastal forntogenesis. Quart. J. Roy. Meteor. Soc., 101, 957-978.

Bottema, M., Van Vledder, G.Ph., 2008. Effective fetch and non-linear four-wave interactions during wave growth in slanting fetch conditions. Coastal Engineering 55 (2008) 261-275.

Breivik, Ø, J-R Bidlot and PAEM Janssen (2016). A Stokes drift approximation based on the Phillips spectrum, Ocean Modell., 100, pp 49-56, doi:10.1016/j.ocemod.2016.01.005, arXiv:1601.08092

Breivik, Ø, K Mogensen, J-R Bidlot, MA Balmaseda and PAEM Janssen (2015). Surface Wave Effects in the NEMO Ocean Model: Forced and Coupled Experiments, J Geophys Res: Oceans, 120,doi:10.1002/2014JC010565

Breivik, Ø, PAEM Janssen and J-R Bidlot (2014). Approximate Stokes Drift Profiles in Deep Water, J PhysOceanogr, 44, 2433-2445, doi:10.1175/JPO-D-14-0020.1

Bretschneider, C.L., (1958). Revisions in wave forecasting: Deep and shallow water, Proc. 6th Conf. On Coastal Eng., ASCE, Council on Wave Research.

Bretschneider, D.L., 1952. Revised wave forecasting relationships, Proceedings 2nd Conference on Coastal Engineering, ASCE, Council on Wave Research.

Broström, G, K H Christensen, M Drivdal, J E Weber (2014). Note on Coriolis-Stokes force and energy, Ocean Dyn, 64(7), pp 1039-1045, doi:10.1007/s10236-014-0723-8

Brown, J.M., R.Bolanos, and J.Wolf, 2013. The depth-varying response of coastal circulation and water level in $2 \mathrm{D}$ radiation stress when applied in a coupled wave-tide-surge modeling system during an extreme storm, Coastal Eng., 82, 102-113.

Brown, J.M., Wolf, J., 2009, Coupled wave and surge modelling for the eastern Irish Sea and 
implications for model wind-stress. Continental Shelf Research, 29, 1329-1342.

Bunney, C., A.Saulter, 2016, An ensemble forecast system for prediction of Atlantic-UK wind waves, Ocean Modelling, doi:10.1016/j.ocemod2015.07.065.

Burke, J. (2008). The one I'll never forget, Bayview Yacht Club Report, www.bycmack.com/history/1985_Port_Huron_to_Mack_Race_by_John_Burke_(2008).pdf,

Carniel S., A. Benetazzo, D. Bonaldo, F.M. Falcieri, M.M. Miglietta, A. Ricchi, and M. Sclavo (2016) Scratching beneath the surface while coupling atmosphere, ocean and waves: analysis of a dense water formation event. Ocean Model. DOI: 10.1016/j.ocemod.2016.03.007.

Cavaleri, L. and L. Bertotti, (2004). Accuracy of the modeled wind and wave fields in enclosed seas, Tellus, 56A, 167-175.

Cavaleri, L. and M.Sclavo, 1998. Characteristics of quadrant and octant advection schemes in wave models, Coastal Engineering, 34, 221-242.

Cavaleri, L., 1980, Wave measurements using a pressure transducer, OceanologicaActa, Vol.3, N.3, 339-346.

Cavaleri, L., 2000. The oceanographic tower Acqua Alta - activity and prediction of sea states at Venice, Coast. Eng., 39, 29-70.

Cavaleri, L., 2006. Wave modeling - where to go in the future, Bull. Amer. Meterol. Soc., vol. 87, no. 2, pp. 207-214.

Cavaleri, L., and L.Bertotti, 1997. In search of the correct wind and wave fields in a minor basin, Monthly Weather Review, 125, 8, 1964-1975.

Cavaleri, L., and L.Bertotti, 2006. The improvement of modelled wind and wave fields with increasing resolution, Ocean Engineering, 33, 5-6, 553-565.

Cavaleri, L., and S.Zecchetto, 1987. Reynolds stresses under wind waves, J.Geoph.Res., April 15, Vol.92, No. C4, 3894-3904.

Cavaleri, L., B. Fox-Kemper, and M. Hemer, (2012). Wind waves in the coupled climate system, BAMS, 93(11), 1651-1661.

Cavaleri. L., J.-H.G.M.Alves, F.Ardhuin, A.Babanin, M.Banner, K,Belibassakis, M.Benoit, M.Donelan, J.Groeneweg, T.H.C.Herbers, P.Hwang, P.A.E.M.Janssen, T.Janssen, I.V.Lavrenov, R.Magne, J.Monbaliu, M.Onorato, V.Polnikov, D.Resio, W.E.Rogers, A.Sheremet, J.McKee Smith, H.L.Tolman, G.van Vledder, J.Wolf, and I.Young, 2007. Wave modelling - the state of the art, Progress in Oceanography, 75, Issue 4, 603-674, doi:10.1016/j.pocean.2007.05.005.

Cavaleri, L., L.Bertotti, R.Buizza, A.Buzzi, V.Masato, G.Umgiesser, and M.Zampato 2010. Predictability of extreme meteo-oceanographic events in the Adriatic Sea, Quart. Jour. Roy.Meteor.Soc., Feb 2010, pp.400-413 DOI: 10.1002/qj.567

Chawla, A., and J.T. Kirby. 2002. Monochromatic and random wave breaking at blocking points. J. Geophys. Res.-Oceans, 107(C7), 3067. 
Chawla, A., Tolman, H.L., Gerald, V., Spindler, D., Spindler, T., Alves, Jose-Henrique G. M.; Cao, Degui; Hanson, Jeffrey L.; Devaliere, Eve-Marie, 2013. A Multigrid Wave Forecasting Model: A New Paradigm in Operational Wave Forecasting, Weather \& Forecasting, 28(4), 1057-1078

Cipollini, P., Benveniste, J., Bouffard, J., Emery, W., Fenoglio-Marc, L., Gommenginger, C., Griffin, D., Hoyer, J., Kurapov, A., Madsen, K., Mercier, F., Miller, L., Pascual, A., Ravichandran M., Shillington, F., Snaith, H., Strub, P.T., Vandenmark, D., Vignudelli, S., Wilkin, J., Woodworth, P., Zavala-Garay, J., 2009. The role of altimetry in coastal observing systems, Proceedings of the OCEANOBS'09 Conference, WSA WPP-306, Vol. 2(I), 181-192.

COASTALT, 2011. Development of Radar Altimetry Data Processing in the Coastal Zone, http://www.coastalt.eu/ or http:/www.coastalaltimetry.org.

Colby, F.P., (2004). Simulation of the new England sea breeze: the effect of grid spacing, Wea. Forecasting, 19, 277-285.

Collins III, C.O., Vincent, C.L., Graber, H.C., 2015. A statistical method for correlating paired wave spectra, Journal of Atmospheric and Oceanic Technology, Vol 32, 2130-2146.

Collins, C.O, W. E. Rogers, A. Marchenko and A. V. Babanin, 2015. In situ measurements of an energetic wave event in the Arctic marginal ice zone, Geophys. Res. Lett., 42, doi:10.1002/2015GL063063.

Collins, C.O., Lund, B., Waseda, T., Graber, H.C., 2014. On recording sea surface elevation with accelerometer buoys: lessons from ITOP (2010). Ocean Dynamics 64:6, 895-904.

Collins, J.I., 1972. Prediction of shallow water spectra, J. Geophys. Res., 77, No. 15, 2693-2707.

Corbella S., Pringle J., Stretch D., 2015: Assimilation of ocean wave spectra and atmospheric circulation patterns to improve wave modelling, Coastal Engineering, 100, 1-10, ISSN 0378-3839, http://dx.doi.org/10.1016/j.coastaleng.2015.03.003.

Courant, R., Friedrichs, K., and Lewy, H., 1928, On the partial difference equations of mathematical physics, IBM Journal of Research and Development, 11, 215-234.

Craig, P. D. (1996), Velocity profiles and surface roughness under breaking waves, J. Geophys. Res., 101(C1), 1265-1277, doi:10.1029/95JC03220

Craig, P. D., and M. L. Banner (1994), Modeling wave-enhanced turbulence in the ocean surface layer, J. Phys. Oceanogr., 24(12), 2546-2559,doi:10.1175/15200485(1994)024<2546:MWETIT>2.0.CO;2.

Craik, A and S Leibovich (1976). A rational model for Langmuir circulations, J Fluid Mech, 73(03), pp 401-426, doi:10.1017/S0022112076001420

Dabbi, E.P., Haigh, I.D., Lambkin, D., Hernon, J., Williams, J.J., Nicholls, R.J., 2015. Beyond significant wave height: A new approach for validating spectral wave models, Coastal Engineering, Vol 100, 11-25.

Daley, R., 1993, Atmospheric data analysis, Cambridge University Press, ISBN 9780521458252, pp. 
Dalrymple, R.A., Kirby, J.T., Hwang, P.A., 1984. Wave diffraction due to areas of energy dissipation. J. Waterw. Port Coast. Ocean Eng. 110, 67-79.

Dee, D.P., S.M.Uppala, A.J.Simmons, P.Berrisford, P.Poli, S.Kobayashi, U.Andreae, M.A.Magdalena, G.Balsamo, P.Bauer, P.Bechtold, A.C.M.Beljaars, L.van de Berg, J.Bidlot, N.Borgmann, C.Delsol, R.Dragani, M.Fuentes, A.J.Geer, L.Haimberger, S.B.healy, E.V.Holm, L.Isaksen, P.Källberg, M.Köhler, M.Matricardi, A.P.Mc Nally, B.M.Monte-Sanz, J.-J.Morcrette, B.K.Park, C.Peubey, P.de Rosnay, C.Tavolato, J.-N.Thépaut, and F.Vitart, 2011. The ERA-Interim reanalysis: configuration and performance of the data assimilation system, Quart. J. Roy. Met. Soc., 137, 553-597, doi: 10.1002/qj.828

Dietrich, J.C., S. Tanaka, J.J. Westerink, C.N. Dawson, R.A. Luettich, M. Zijlema, L.H. Holthuijsen, J.M. Smith, L.G. Westerink, and H.J. Westerink. 2012. Performance of the Unstructured-Mesh, SWAN+ADCIRC Model in Computing Hurricane Waves and Surge. J. Sci. Comput., 52(2), 468497.

Dietrich, J.C., Zijlema, M., Allier, P.E., Holthuijsen, L.H., Booij, N., Meixner, J.D., Proft, J.K., Dawson, C.N., Bender, C.J., Naimaster, A., Smith, J.M., Westerink, J.J, 2013. Ocean Modelling 70, 85-102.

Dietrich, J.C., Zijlema, M., Westerink, J.J., Holthuijsen, L.H., Dawson, C., Luettich Jr., R.A., Jensen, R.E., Smith, J.M., Stelling, G.S., Stone, G.W., 2011. Modeling hurricane waves and storm surge using integrally-coupled, scalable computations. Coastal Engineering 58, 45-65.

Doble, M. J., and J.-R. Bidlot, 2013. Wavebuoy measurements at the Antarctic sea ice edge compared with an enhanced ECMWF WAM: progress towards global waves-in-ice modeling, Ocean Model., 70, 166-173, doi:10.1016/j.ocemod.2013.05.012.

Dodet, G., X. Bertin, N. Bruneau, A. B. Fortunato, A. Nahon, and A. Roland (2013), Wave-current interactions in a wave-dominated tidal inlet, J. Geophys. Res. Oceans, 118, 1587-1605, doi: $10.1002 /$ jgrc.20146.

Donelan, M.A., Dobson, F.W., Graber, H.C., Madsen, N., McCormick, C., 2005. Measurement of wind waves and wave-coherent air pressures on the open sea from a moving SWATH vessel, J. Atmos. Oceanic Technol., 22, 896-908.

Donelan, M.A., Hamilton, J., Hui, W.H., 1985. Directional wave spectra of wind generated waves. Philos. Trans. Roy. Soc. London, Ser A, 315, 500-562.

Donelan, M.A., Skafel., M., Graber, H., Liu, P., Schwab, D. and Venkatesh, S., 1992. On the growth rate of wind-generated waves, Atmospheric-Ocean, 30, 457-478.

Doyle, J.D., and T.T. Warner, (1993). Nonhydrostatic simulations of coastal mesobeta-scale vortices and frontogenesis. Mon. Wea. Rev., 121, 3371-3392.

Drennan, W.M., Donelan, M.A., Madsen, N., Katsaros, K.B., Terray, E.A., Flagg, C.N., 1994. Directional wave spectra from a swath ship at sea, J. Atmos. Oceanic Technol., 11, 1109-1116. Drennen, W.M., Graber, H.C., Collins III, C.O., Herrera, A., Potter, H., Ramos, R.J., Williams, N.J., 2014. EASI: An air-sea interaction buoy for high waves, Journal of Atmospheric and Oceanic 
Technology, Vol. 31, 1397-1409.

Durrant, T.H., Greenslade, D.J.M., Simmonds, I., 2009. Validation of Jason-1 and Envisat remotely sensed wave heights, J. Atmos. Oceanic Technol., 26, 123-134.

Eldeberky, Y., and J.A.Battjes, 1995. Parametrisation of triad interactions in wave energy model. Proc. Coastal Dynamics Conf. '95, Gdansk, Poland, 140-148.

Elias, E.P.L., G. Gelfenbaum, and A.J. van der Westhuysen. 2012. Validation of a coupled waveflow model in a high-energy setting: The mouth of the Columbia River. J. Geophys. Res.-Oceans, 117(C9), 2156-2202. doi:10.1029/2012JC008105 .

Engelstad, A., Jansen, T.T., Herbers, T.H.C., van Vledder, G.Ph., Elgar, S., Raubenheimer, B., Trainor, L., Garcia-Garcia, A., 2013. Wave evolution across the Louisiana shelf. Continental Shelf Res. 52, 190-202.

Fan, Y., and S. M. Griffies (2014), Impacts of parameterized Langmuir turbulence and non-breaking wave mixing in global climate simulations, J. Clim., 27, 4752-4775, doi:10.1175/JCLI-D-1300583.1.

Fan, Y., I. Ginis, T. Hara, C.W. Wright, and E.J. Walsh. 2009. Numerical Simulations and Observations of Surface Wave Fields under an Extreme Tropical Cyclone. J. Phys. Oceanogr. 39, 2097-2116.

Fer, Ilker, and Mostafa Bakhoday Paskyabi (2014) Autonomous ocean turbulence measurements using shear probes on a moored instrument. Journal of Atmospheric and Oceanic Technology 31, 474-490.

Ferrarin, C., G. Umgiesser, A. Cucco, T.-W. Hsu, A. Roland, and C. L. Amos, 2008, Development and validation of a finite element morphological model for shallow water basins, Coastal Eng., 55(9), 716-731, doi:10.1016/j.coastaleng.2008.02.016.

Ferrarin, C., Roland, A., Bajo, M., Umgiesser, G., Cucco, A., Davolio, S., Buzzi, A., Malguzzi, P., and Drofa, O., 2013, Tide surge wave modelling and forecasting in the Mediterranean Sea with focus on the Italian coast, Ocean Modelling, 61, 38-48, doi: 10.1016/j.ocemod.2012.10.003.

Filipot, J.F., Ardhuin, F., Babanin, A.V., 2010. A unified deep-to-shallow water wave-breaking probability parameterization, J. Geophys. Res., 115, C04022, doi:10.1029/2009JC005448.

Flamant, C. J. Pelon, D. Hauser, C. Quentin, W. M. Drennan, F. Gohin, B. Chapron, and J. Gourrion, (2003). Analysis of surface wind and roughness length evolution with fetch using a combination of airborne lidar and radar measurements, J. Geophy. Res, 108, No. C3, 8058.

Forristal, G.Z., and A.M.Reece, 1985. Measurements of wave attenuation due to a soft bottom: the SWAMP experiment, J. geoph. Res., C90, 3376-3380.

Forristall, G.Z., E.H.Doyle, W.Silva, and M.Yoshi, 1990. Verification of a soil wave interaction model (SWIM), p41-68 in: Modeling Marine Systems, Vol.II, A.M.Davies (ed); CRC Press, Boca Raton, Florida.

Fredreickson, P.A. and K.L. Davidson, (2003). Observational buoy studies of coastal air-sea fluxes, J. Climate, 16, 593-599. 
Freilich, M.H., Guza, R.T., and Elgar, S.L., 1990. Observations of nonlinear effects in directional spectra of shoaling waves, Journal of Geophysical Research, Vol 95, No. C6, 9645-9656.

Fujita, T.T., 1981. Tornadoes and downbursts in the context of generalized planetary scales, J. Atmos Sci., 38(8), 1511-1534.

Garcia-Nava, H., F.J. Ocampo-Torres, P. Osuna, nd M.A. Donelan, (2009). Wind stess in the presence of swell under moderate to strong wind conditions, J. Geophy. Res, 114 (C23008).

Gautier, C. and S.Caires, 2015. Operational wave forecasts in the southern North Sea, IAHR 2015, The Hague, the Netherlands, 28 June-3 July, 4pp.

Geiser, J., 2012, Iterative splitting methods for solving time-dependent problems, Applied Mathematics Letters, vol. 25, no. 5, pp. 793-797.

Gemmrich, J. and C. Garrett,, (2012). The signature of inertial and tidal currents in offshore wave records, J. Phys. Oceanogr., 42, 1051-1056.

Gemmrich, J. and D. M. Farmer, 1999: Near-surface turbulence and thermal structure in a winddriven sea. J. Phys. Oceanogr., 29, 480-499.

Gemmrich, J. R., T. D. Mudge, and V. D. Polonichko. On the energy input from wind to surface waves, Journal of Physical Oceanography 24.11 (1994): 2413-2417.

Gerling T.W., 1992. Partitioning Sequences and Arrays of Directional Ocean Wave Spectra into Component Wave Systems. J. Atmos. Oceanic Technol., 9, 444-458. , doi:10.1175/15200426(1992)009<0444:PSAAOD>2.0.CO;2

Gerritsen, H., E.D. de Goede, F.W. Platzek, M. Genseberger, J.A.Th.M. van Kester, and R.E. Uittenbogaard. 2007. Validation Document Delft3D-FLOW. Report X0356, M3470, Delft Hydraulics, 109 pp.

Gille, S.T., S. G. Llewellyn Smith, and N. M. Statom, (2005). Global observations of the land breeze, Geophy. Res. Letters, Vol. 32, L05605.

Godunov, S, 1959, A, finite-difference method for the numerical computation of discontinuous solutions of the equations of fluid dynamics, Mat. Sb., 47 (1959), pp. 357-393.

Graber, H.C., 2005: The shoaling wave experiment. J. Atmos. Oceanic Technol., 22, 797-797.

Graber, H.C., E.A. Terrary, E.A., Donelan, M.A., Drennan, W.M., van Leer, J.C., Peters, D.B., 2000. ASIS-A new air-sea interaction spar buoy: Design and performance at sea. J. Atmos. Oceanic Technol., 17(5), 708-720.

Grant, A. L., and S. E. Belcher (2009), Characteristics of Langmuir turbulence in the ocean mixed layer, J. Phys. Oceanogr., 39, 1871-1887,doi:10.1175/2009JPO4119.1

Groeneweg, J., A.J. van der Westhuysen, G.P. van Vledder, S. Jacobse, J. Lansen, and A.R. van Dongeren. 2008. Wave modelling in a tidal inlet: performance of SWAN in the Wadden Sea. Proc. 31th Int. Conf. Coastal Eng., ASCE, 411-423. 
Groeneweg, J., M.van Gent, J.van Nieuwkoop, and Y.Toledo, 2015. Wave propagation in complex coastal systems and the role of nonlinear interactions, J. Waterways, Port, Coastal, Ocean Eng., 141, 5, 17pp, doi.org/10.1061/(ASCE)WW.1943-5460.0000300

Hamilton, R.C., 1972. Ocean data gathering program report No. 7 covering hurricane Edith September 16, 1971, Baylor Company, Houston Texas.

Hanson, J. L., Phillips, O.M., 2001. Automated analysis ofocean surface directional wave spectra, Journal of Atmospheric and OceanicTechnology., 18, 277-293.

Harcourt, R.R (2015). An Improved Second-Moment Closure Model of Langmuir Turbulence, J Phys Oceanogr, 45(1), pp 84-103, doi:10.1175/JPO-D-14-0046.1

Harcourt, R R and E.A.D'Asaro (2008). Large-Eddy Simulation of Langmuir Turbulence in Pure Wind Seas, J Phys Oceanogr, 38(7), pp 1542-1562, doi:10.1175/2007JPO3842.1

Harcourt, R. R., 2013: A second moment closure model of Langmuir turbulence. J. Phys. Oceanogr., 43, 673-697.

Hasselmann, K. 1971. On the mass and momentum transfer between short gravity waves and largerscale motions. J. Fluid Mech., 50, 189-201.

Hasselmann, K., and Collins, J.I., 1968. Spectral dissipation of finite-depth gravity waves due to turbulent bottom friction, J. Mar. Res., 26, 1-12.

Hasselmann, K., Barnett, T.P., Bouws, E., Carlson, H., Cartwright, D.E., Enke., K., Ewing, J.A., Gienapp., H., Hasselnamm, D.E., Kruseman, P., Meerburg, A., Muller., P., Olbers, D.J., Richter, K., Sell, W., and Walden, H., 1973. Measurements of wind-wave growth and swell decay during the Joint North Sea Wave Project, (JONSWAP), Dtsch. Hydrogr. Z. Suppl. A 8(12), 95p.

Hasselmann, S., and K.Hasselmann, 1981. A symmetrical method of computing the nonlinear transfer in a gravity wave spectrum. Hamburger Geophysiklische Einzelschriften, Reihe A: Heft 52. Herbers, T.H.C., Jessen, P.F., Janssen, T.T., Colbert, D.B., MacMahan, J.H., 2012. Observing ocean surface waves with GPS-tracked buoys, J. Atmos. OceanicTechnol., 29, 944-959.

Herbers, T.H.C., S.J. Lentz S.J., 2010. Observing directional properties of ocean swell with an acoustic doppler current profiler (ADCP), J. Atmos. Oceanic Technol., 27, 210-225.

Herbers. T.H.C., Hendrickson, E.J., O’Reilly, W.C., 2000. Propagation of swell across a wide continental shelf, . Geophy. Res. Vol 105, No. C3, 19,729-19,737.

Hersbach, H. and Janssen, P.A.E.M., 1999, Improvement of the short-fetch behaviour in the Wave Ocean Model (WAM), Journal of Atmospheric and Oceanic Technology, 16, 884-892.

Herterich, K., and K.Hasselmann, 1980. A similarity relation for the nonlinear energy transfer in a finite-depth gravity-wave spectrum. J. Fluid Mech., 97, 215-224.

Holland, K. T., Elmore, P.A., 2008. A review of heterogeneous sediments in coastal environments, Earth-Science Reviews, 89 (Issue 3-4), 116-134. 
Holland, K.T., S.B.Vinzon, and L.I.Callian, 2009. A field study of coastal dynamics on a muddy coast offshore of Cassino beach, Brazil, Cont. Shelf Res., 29, 3, 503-514

Holthuijsen L-H., Herman A., Booij N., 2003. Phase-decoupled refraction-diffraction for spectral wave models. Coastal Eng49:291-305.

Holthuijsen, L., 2007: Waves in oceanic and coastal waters, Cambridge University Press, pp ISBN: 978-0521129954, pp. 387.

Holthuijsen, L.H, and H.L. Tolman. 1991. Effects of the Gulf Stream on ocean waves. Journal of Geophys. Res.-Oceans, 96(C7), 12,755-12,771.

Hope, M.E., Westerink, J.J., Kennedy, A.B., Kerr, P.C., Dietrich, J.C., Dawson, C., Bender, C.J., Smith, J.M., Jensen, R.E., Zijlema, M., Holthuijsen, L.H., Luettich, R.A., Powell, M.D., Cardone, V.J., Cox, A.T., Pourtaheri, H., Roberts, H.J., Atkinson, J.H., Tanaka, S., Westerink, H.J., and Westerink, L.G., 2013, Hindcast and validation of Hurricane Ike (2008) waves, forerunner, and storm surge, JGR - Oceans, 118, 4424-4460.

Hsu, T.W., Ou, S.H. and Liau, J.M., 2005a. Hindcasting near shore wind waves using a FEM code for SWAN. Coastal Engineering, 52, 177-195.

Hsu, T.W., Ou, S.H., Liau, J.M., Zanke, U., Roland, A. and Mewis, P., 2005b. Verification and development of a spectral finite element wave model. Waves 2005, ASCE/COPRI, The Fifth International Symposium on Wave Measurement and Analysis. Ed. Billy Edge, Madrid, Spain. Hsu, Y.L., J.D. Dykes, R.A. Allard, and D.W. Wang. 2008. Validation Test Report for Delft3D. NRL/MR/7320 — 08-9079, Naval Research Lab, Stennis Space Center, MS, 42pp.

Huang, C. J., F. Qiao, Z. Song, and T. Ezer (2011), Improving simulations of the upper ocean by inclusion of surface waves in the Mellor-Yamada turbulence scheme, J. Geophys. Res., 116, C01007, doi:10.1029/2010JC006320

Huchet, M., Leckler, F., Filipot, J.-F., Roland, A., Ardhuin, F., Sikiric, M.D., Michaud, H., Delpey, M.T., Dodet, G.,2015. On the high resolution coastal application with WAVWATCH III. Proc. 14th Workshop on Wave Hindcasting and Forecasting, Key West, Florida, USA.

Huchet, M., Leckler, F., Filipot, J-F., Roland, A., Ardhuin, F., DutourSikiric, M., Michaud, H., Delpey, M.T. and Dodet, G., 2015, High resolution modelling of nearshore wave processes using new implicit scheme of WAVEWATCH III, 14th International Workshop on Wave Hindcasting and Forecasting \& 5th Coastal Hazard Symposium.

Hultquist, T.R., Dutter, M.R. and D.J. Schwab, (2006). Reexamination of the 9-10 November 1975 "Edmund Fitzgerald" Storm using today's technology, AMS, BAMS, 87 (5), 607-622.

Hwang, P.A., Wang, D.W., Walsh, E.J., Krabill, W.B., Smith,R.N., 2000. Airborne measurements of the wave number spectra of ocean surface waves. Part I: Spectral slope and dimensionless spectral coefficient, J. Phys. Oceanogr., 30, 2753-2767.

IOOS, 2009. A national operational wave observation plan. Integrated Ocean Observing System Program Office, Silver Spring, MD, 76pp,

(http://www.ioos.noaa.gov/library/wave_plan_final_03122009.pdf ).

Isoguchi, O. and H. Kawamura, (2007). Coastal wind jets flowing into the Tusushima strait and their 
effect on wind-wave development, J. Atmos. Sci, 64, 564-578.

Jacob, R., J. Larson, and E. Ong. 2005. MxN communication and parallel interpolation in Community Climate System Model Version 3 using the model coupling toolkit. Int. J. High Perform. C., 19, 293-307.

Janssen P.A.E.M, Abdalla S., Hersbach H., and Bidlot J.R., 2007. Error Estimation of Buoy, Satellite, and Model Wave Height Data. J. Atmos. Oceanic Technol., 24, 1665-1677, doi: http://dx.doi.org/10.1175/JTECH2069.1

Janssen, P.A.E.M, Abdalla, S., Hersbach, H., 2003. Error estimation of buoy, satellite and model wave height data, Tech. Memorandum 402, European Center for Medium Range Weather Forecasts, Reading UK.

Janssen, P.A.E.M., (2012), Ocean wave effects on the daily cycle in SST, J. Geophys. Res., 117, C00J32, doi:10.1029/2012JC007943.

Janssen, P.A.E.M., O. Saetra, C. Wettre, H. Hersbach, and J. Bidlot (2004), Impact of the sea state on the atmosphere and ocean, in AnnalesHydrographiques, vol. 3-772, pp. 3.1-3.23, Serv. Hydrogr. etOc_eanogr. de la Mar.

Janssen, P.A.E.M., (1991). Quasi-linear theory of wind wave generation applied to wave forecasting, J. Phys. Oceanogr., 19, 745-754.

Janssen, P.A.E.M., (2004). The Interaction of Ocean Waves and Wind, Cambridge University Press, 300pp.

Janssen, P.A.E.M., 2008. Progress in ocean wave forecasting, Journal of Computational Physics

Janssen, P.A.E.M., and P.Viterbo, 1996. Ocean waves and the atmospheric climate, J.Climate, 9, 1269-1287.

Janssen, P.A.M.E, B. Hansen, B., Bidlot, J.-R., 1997. Verification of the ECMWF wave forecasting system against buoy and altimeter data, Wea. Forecasting, 12, 763-784.

JCOMM, 2008. Final Report of the JCOMM Technical Workshop on Wave Measurements from Buoys, JCOMM-TR - 047, WMO/TD-No. 1466, IOC-NO. 208, IOC Workshop Report No. 208, New York, NY, October 2008, http://boram.lee.f.free.fr/data/J-TR-47-Wave-Buoy-Workshop/ .

Jenkins, A. D., 1987: Wind and wave induced currents in a rotating sea with depth- varying eddy viscosity. J. Phys. Oceanogr., 17, 938-951.

Jensen, R.E., M.A. Cialone, R.S. Chapman, B.A. Ebersole, M. Anderson, and L. Thomas, (2012). Lake Michigan Storm: wave and water level modeling, Great Lakes Coastal Flood Study, 2012 Federal Inter-Agency Initiative, ERDC/CHL TR-12-26, USACE Engineer Research and Development Center, Vicksburg, MS, 330p.

Jensen, R.E., Swail, V., Lee, B., O'Reilly, W.A., 2011. Wave measurement evaluation and testing, , Proceedings of 12th International Workshop on Wave Hindcasting and Forecasting, Kohala Coast, Hawaii. 
Jensen, R.E., Swail, V.R., Buchard, R.H., Reily, R.E., Hesser, T.J., Blaseckie, M., MacIsaac. C., 2015. Field laboratory for ocean sea state investigation: FLOSSIE intra-measurement evalustion of $6 \mathrm{~N}$ wave buoy systems, Proceedings of the 14th International Workshop on Wave Hindcasting and Forecasting, Key West, Florida, 8-13 November 2015.

Jiang, A., (2012). A linear theory of three-dimensional land-sea breezes, J. Atmos. Sci.,69, 18901909.

Jonsson, I.G. 1990. Wave-current interactions. Chapter 3 in The Sea, Vol. 9, Part A, edited by Le Mehaute and Hanes. John Wiley \& Sons, 65-120.

Kahma, K.K., and C.J.Calkoen, 1992. Reconciling discrepancies in the observed growth of windgenerated waves, J. Phys. Oceanogr., 22, 1389-1405.

Kalnay, E., 2003, Atmospheric Modeling, Data Assimilation and Predictability, Cambridge University Press, Cambridge, 2003. pp. 364, ISBN: 9780521796293

Kantha, L.K., and C.A. Clayson. 2004. On the effect of surface gravity waves on mixing in the oceanic mixed layer. Ocean Model., 6(2), 101-124.

Kashino, R., 2011. Session III-Recent advances and issues in wave buoy technologies, Proc. Ocean Waves Workshop 2011, New Orleans, LA (http://scholarworks.uno.edu/oceanwaves/2011/Session3/1/ )

Kennedy, A.B., S. Rogers, A. Sallenger, U. Gravois, B. Zachry, M. Dosa, and F. Zarama. 2011. Building destruction from waves and surge on the Bolivar Peninsula during Hurricane Ike, J. Waterw. Port Coast., 137(3), 132-141.

Kirby, J., 2003. Boussinesq Models and Applications to Nearshore Wave Propagation, Surf Zone Processes and Wave-induced Currents. Advances in Coastal Modeling, ed. C. Lakhan, Elsevier Sciences, $41 \mathrm{pp}$.

Kirby, J.T., and T.M. Chen. 1989. Surface waves on vertically sheared flows: approximate dispersion relations. J. Geophys. Res.-Oceans, 94, 1013-1027.

Kleijweg, J.C.M., Van Vledder, G.Ph., Steeghs, T.P.H., 2005. Integration of X-band remote sensing and numerical wave modelling of waves. Proc. Waves2005, Madrid, Spain.

Komen, G. J., L. Cavaleri, M. Donelan, K. Hasselmann, S. Hasselmann and P. A. E. M. Janssen, 1994: Dynamics and Modelling of Ocean Waves, Cambridge University Press, Cambridge. ISBN: 9780521577816, pp 556.

Komen, G.J., Hasselmann, S., Hasselmann, K., 1984. On the existence of a fully developed wind-sea spectrum. J. Phys. Oceanogr. 14, 1271-1285.

Krogstad, H.E., Wolf, J., Thompson, S.P., Wyatt, L.R., 1999. Methods for intercomparion of wave measurements, Coastal Engineering 37, 235-257.

Kumar, N., G. Voulgaris, J.C. Warner, and M. Olabarrieta. 2012. Implementation of the vortex force formalism in the coupled ocean-atmosphere-wave-sediment transport (COAWST) modeling system for inner shelf and surf zone applications, Ocean Model., 47, 65-95. 
Kumar, N., Voulgaris, G., and Warner, J.C. (2011). Implementation and modification of a threedimensional radiation stress formulation for surf zone and rip-current applications, Coastal Engineering, 58, 1097-1117, doi:10.1016/ j.coastaleng.2011.06.009.

Lai, R.J., S.R. Long, and N.E. Huang. 1989. Laboratory studies of wave-current interaction: Kinematics of the strong interaction, J. Geophys. Res.-Oceans, 94, 16,201-16,214.

Landsea, C. W., S. Feuer, A. Hagen, D. A. Glenn, J. Sims, R. Perez, M. Chenoweth, and N. Anderson, 2012: A reanalysis of the 1921-1930 Atlantic hurricane database. Journal of Climate, 25, 865-885.

Langodan, S., Cavaleri, L., Anadhapalli, Y.V., Hoteit, I., 2014. The Red Sea: A natual laboratory for wind and wave modelling. Journal of Phys. Oceanogr., 44, 3139-3159. DOI: 10.1175/JPO-D-130242.1.

Langodan, S., L. Cavaleri, Y. Viswanadhapalli, and I. Hoteit, 2014: The Red Sea: a natural laboratory for wind and wave modeling. J. Phys. Oceanogr. doi:10.1175/JPO-D-13-0242.1, in press.

Langodan, S., L.Cavaleri, V.Viswanadhpaili, and I.Hoteit, 2015. Wind-wave source functions in opposing seas, J.Geoph.Res., doi 10.1002/2015JC010816, 6751-6768.

Lanser, D. and Verwer, J.G., 1999: Analysis of operator splitting for advection-diffusion-reaction problems from air pollution modelling. J. Comput. Appl. Math. 111, 201-216.

Larson, J., Jacob, R., and Ong, E. 2004. The model coupling toolkit: A new Fortran90 toolkit for building multiphysics parallel coupled models. Int. J. High Perform. C., 19, 277-292.

Lax, P.D. and Wendroff, B., 1960, Systems of conservation laws. Commun. Pure Appl Math., 13, 217-237.

Lax, P.D., and R.D.Richtmyer, 1956.Survey of the stability of linear finite difference equations.Comm. Pure Appl. Math., 9 (1956), 267-293, doi:10.1002/cpa.3160090206

Lesser, G.R., J.A. Roelvink, J.A.T.M. van Kester, and G.S. Stelling. 2004. Development and validation of a three-dimensional morphological model. Coast. Eng., 51 (8-9), 883-915.

LeVeque R. J. and H. C. Yee, 1990, A study of numerical methods for hyperbolic conservation laws with stiff source terms, J. Comp. Phys. 86, 187

Li, J.-G., Holt, M., 2009. Comparison of Envisat ASAR ocean wae spectra with buoy and altimeter data via a wave model, J. Atmos. Oceanic Technol., 26, 593-614.

Li, J-G., 2012, Propagation of ocean surface waves on a spherical multiple-cell grid. J. Comput. Phys., 231:8262-8277.

Li, Q, A Webb, B Fox-Kemper, A Craig, G Danabasoglu, W G Large, M Vertenstein (2015). Langmuir mixing effects on global climate: WAVEWATCH III in CESM, Ocean Modell, doi:10.1016/j.ocemod.2015.07.020

Li, Q, B Fox-Kemper, Ø Breivik, A Webb (2017). Statistical Models of Global Langmuir Mixing, 
Ocean Model, 113, pp 95-114, doi:10.1016/j.ocemod.2017.03.016

Liau J-M., Roland A., Hsu T-W., Ou S-H-, Li Y-T., 2011, Wave refractiondiffraction effect in the wind wave model WWM. Coastal Eng, 58:429-443

Liau, J-M. 2001, A Study of Wind Waves Hindcasting on the Coastal Waters, PhD Thesis, National, Cheng Kung University, Tainan, Taiwan

Lionello, P., H. Günther and P. A. E. M. Janssen, 1992. Assimilation of altimeter data in a global third-generation wave model, Journal of Geophysical Research, 97(14), 453-474.

doi:10.1029/92JC01055.

Loescher, K. A., G. S. Young, B.A. Colle, and N. S. Winstead, 2006. Climatology of barrier jets along the Alaskan coast. Part I: Spatial and temporal distributions, Monthly Weather Review 134, 437-453.

Long, C.E., and Oltman-Shay, J.M., 1991. Directional characteristics of waves in shallow water, US Army Corps of Engineers, Coastal Engineering Research Center, Technical Report CERC-91-1, USACE, Vicksburg, Mississippi, 152p.

Longuet-Higgins, M.S., and R.W. Stewart. 1960. Changes in form of short gravity waves on long tidal waves and tidal currents. J. Fluid Mech., 8, 565-583.

Longuet-Higgins, M.S., and R.W. Stewart. 1961. The changes in amplitude of short gravity waves on steady non-uniform currents. J. Fluid Mech., 10, 529-549.

Longuet-Higgins, M.S., and R.W. Stewart. 1962. Radiation stress and mass transport in gravity waves, with applications to "surf beats". J. Fluid Mech., 13, 481-504.

Longuet-Higgins, M.S., and R.W. Stewart. 1964. Radiation stresses in water waves: A physical discussion, with applications. Deep Sea Res., 11, 529-562.

Luettich, R.A. Jr., and J.J. Westerink. 2004. Formulation and Numerical Implementation of the 2D/3DADCIRC Finite Element Model Version 44.XX.

http://adcirc.org/adcirc_theory_2004_12_08.pdf

Luther, M.E., Meadows, G., R.Buckley, R., Gilbert, S.A., Purcell, H., Tamburri, M.N., 2013.

Verification of wave measurement systems, Marine Technology Society Journal, Vol. 47, No. 5, 104116.

MacIsaac, C., and S. Naeth, S., 2013. TRIAXYS next wave II directinal wave sensor, The evolution of wave measurements, MTS/EEE Oceans 2013, San Diego, California.

Madec, G and The NEMO team (2012). NEMO Ocean Engine v3.4, Institut Pierre Simon Laplace, 27

Madsen, O.S., Poon, Y.-K., Graber, H.C., 1988. Spectral wave attenuation by bottom friction: Theory, Proc. 21th Int. Conf. Coastal Engineering, ASCE, 492-504.

Madsen, P.A., Sørensen, O.R., 1992. A new form of the Boussinesq equations with improved linear dispersion characteristics. Part 2: A slowly-varying bathymetry, Coastal Engineering, 18, 183-205. 
Magnusson, A.K., Donelan, M.A., 2013. The andrea wave characteristics of a measured north sea rogue wave. J. Offshore Mechanics and Arctic Engineering, 135(3), 031108-1.

Makin, V.K., 2002. A note on the parametrisation of the sea drag. Boundary-Layer Meteorology, 106, 593-600.

Malardel, S., N.Wedi, W.Deconinck, M.Diamantakis, C.Kuhnlein, G.Mozdzynski, M.Hamrud, and P.Smolarkiewicz, 2016. A new grid for the IFS, ECMWF Newsletter, No.146, 23-28.

Malhadas, M., R. Neves, P. Leitao, and A. Silva. 2010. Influence of tide and waves on water renewal in Obidos Lagoon, Portugal. Ocean Dynam., 60, 40-55.

Maresca S, Braca P, Horstmann J, Grasso R., 2014: Maritime surveillance using multiple highfrequency surface-wave radars. IEEE Trans. Geosci. Remote Sens., 52 (8), 5056-5071.

DOI:10.1109/TGRS.2013.2286741

Mass, C.F., M.D. Warner, and R. Steed, (2014). Strong westerly wind events in the strait of Jan de Fuca, Wea. Forecasting, 29, 445-465.

Masson, D., and P.H.LeBlond, 1989. Spectral evolution of wind-generated surface gravity waves in a disperced ice field, J. Fluid Mech., 202, 43-81.

Mastenbroek, C., G. Burgers, and P.A.E.M. Janssen. 1993. The dynamical coupling of a wave model and a storm surge model through the atmospheric boundary layer. J. Phys. Oceanogr., 23 (8), 18561866, doi:10ccb4f9.

McWilliams, J C and P P Sullivan (2000). Vertical mixing by Langmuir circulations, Spill Science and Technology Bulletin, 6(3), pp 225-237, doi:10.1016/S1353-2561

McWilliams, J. C. and J. M. Restrepo, 1999: The wave-driven ocean circulation. J. Phys. Oceanogr., 29, 2523-2540. 1, 2.2.1, 2.2.1

McWilliams, J. C., P. P. Sullivan, and C. H. Moeng, 1997: Langmuir turbulence in the ocean. J. Fluid Mech., 334, 1-30

McWilliams, J.C., J.M. Restrepo, and E.M. Lane. 2004. An asymptotic theory for the interaction of waves and currents in coastal waters. J. Fluid Mech., 511, 135-178.

Mellor, G and A Blumberg (2004). Wave breaking and ocean surface layer thermal response, J PhysOceanogr, 34(3), pp 693-698, doi:10/bftfk9

Mellor, G. 2005. Some consequences of the three-dimensional current and surface wave equations. J. Phys. Oceanogr., 35 (11), 2291-2298.

Mellor, G., 2011. Wave radiation stress. Ocean Dyn., 61 (5), 563-568.

Mendez, F.M., Losada, I.J., 2004. An empirical model to estimate the propagation of random breaking and nonbreaking waves over vegetation fields. Coast. Eng. 51, 103-118. 
Mentaschi, L, Besio, G., Cassola, F. Mazzino, A., 2013. Problems in RMSE-based wave model validations, Ocean Modeling, Vol 72, 53-58.

Mogensen, K S, L Magnusson, J-R Bidlot (2017). Tropical cyclone sensitivity to ocean coupling in the ECMWF coupled model, J Geophys Res: Oceans, 122(5), pp 4392-4412, doi:10.1002/2017JC012753

Monahan, E. C., 1971: Oceanic whitecaps. J. Phys. Oceanogr., 1, 139-144.

Monbaliu, J., Padilla-Henandez, R., Hargreaves, J.C., Albiach, J.C., Luo, W., Sclavo, M. and Guenther, H., 2000, The spectral wave model, WAM, adapted for applications with high spatial resolution, Coastal Engineering, 41, 41-62.

Mukai, A.Y., J.J. Westerink, R.A. Luettich, and D. Mark. 2002. Eastcoast 2001, A tidal constituent database for western North Atlantic, Gulf of Mexico, and Caribbean Sea, ERDC/CHL TR-02-24, Vicksburg, MS, 194 pp.

O'Reilly, W.C., 2007. An introduction to directional wave observations, Wave Sensor Workshop, Alliance for Coastal Technologies, St. Petersburg, Florida, March 7-9 2007.

O’Reilly, W.C., Herbers, T.H.C., Seymour, R.J., Guza, R.T., 1996. A comparison of directional buoy and fixed platform measurements of Pacific swell, . J. Atmos. Oceanic Technol., 13, 231-238.

Ochi, M.K., 2005. Ocean Waves: The Stochastic Approach, Cambridge Ocean Technology Series, Cambridge University Press, 332 pp. ISBN-13: 978-0521017671.

Olabarrieta, M., J.C. Warner, and N. Kumar, N. 2011. Wave-current interaction in Willapa Bay. J. Geophys. Res.-Oceans, 116 (C12014).

Oltman-Shay, J. and Guza R.T., 1984. A data-adaptive ocean wave directional-spectrum estimator for pitch and roll type measurements, Journal of Physical Oceanography, Vol., 14, 1800-1810. Pallares, E., Sanchez-Arcilla, A., Espino, M., 2014. Wave energy balance in wave models (SWAN) for semi-enclosed domains - Application to the Catalan coast. Cont. Shelf. Res., 87, 41-53.

Panteleev, G., Yaremchuk, M and Rogers, W. E.,2015: Adjoint-Free Variational Data Assimilation into a Regional Wave Model. J. Tech 32, 1238-1399. DOI: http://dx.doi.org/10.1175/JTECH-D-1400174.1

Pawka, S.S., 1983. Island shadows in wave directional spectra, Journal of Geophysical Research, Vol. 88, 2579-2591.

Pettersson, H., C.Fortelius, K.Tikka, J.-V.Björkqvist, and K.Kahma, 2014. wave modelling in archipelagos, Coast. Eng., 83, 205-220.

Pettersson, H., Graber, H.C., Hauser, D., Quentin, C., Kahma, K.K., Drennan, W.M.,Donelan, M.A., 2003. Directional wave measurements from three wave sensors during the FETCH experiment, J. Geophys. Res., 108(C3), 8061.

Pezzutto, P., A.Saulter, L.Cavaleri, C.Bunney, F.Marcucci, L.Torrisi, and S.Sebastianelli, 2016, Performance comparison of meso-scale ensemble wave forecasting systems in the Mediterranean Seat, Ocean Modelling, 104, 171-186. 
Phillips, O. M., 1977. The Dynamics of the Upper Ocean, Cambridge Univ. Press, Cambridge, 336pp.

Pierson W. J. (1983). The measurment of synoptic scale wind over the ocean, J. Geophy. Res. Vol. 88, No. C3, 1683-1708.

Plant, W.J, W.C. Keller, and K. Hayes, (2005). Simultaneous measurements of ocean winds and waves with an airborne coherent real aperture radar, . J. Atmos. Oceanic Technol., 22, 832-846. Polton, J. A. (2009), A wave averaged energy equation: Comment on "global estimates of wind energy input to subinertial motions in the Ekman-Stokes layer" by Bin Liu, Kejian Wu and Changlong Guan, J. Oceanogr., 65(5), 665-668, doi:10.1007/s10872-009-0057-1.

Polton, J. A., and S. E. Belcher (2007), Langmuir turbulence and deeply penetrating jets in an unstratified mixed layer, J. Geophys. Res., 112, C09020, doi:10.1029/2007JC004205.

Polton, J. A., D. M. Lewis, and S. E. Belcher (2005), The role of wave-induced Coriolis-Stokes forcing on the wind-driven mixed layer, J. Phys.Oceanogr., 35(4), 444-457, doi:10.1175/JPO2701.1.

Pomaro, A., L.Cavaleri, P.Lionello, 2017. Characteristics and trends of the Adriatic Sea wind waves: analysis of a 37 year long instrument dataset. Submitted for publication.

Popinet, S., R.M.Gorman, G.J.Rickard, and H.LTolman, 2010. A quadtree-adaptive spectral wave model, Ocean Modelling, 34, 1, 36-49.

Portilla, J., 2009. Buoy data assimilation in nearshore wave modelling. PhD Dissertation, Katholieke Universiteit Leuven. pp. 193, ISBN: 9789460180743

Portilla,J., A.L.Caicedo, R.Padilla-Hernandez, and L.Cavaleri, 2015. Spectral wave conditions in the Colombian Pacific Ocean, Ocean Modelling, 92, pp.149-168

Portilla-Yandún J., Cavaleri L., Van Vledder G.Ph., 2015; Wave spectra partitioning and long term statistical distribution, Ocean Modelling, V96, P1, 148-160, doi 10.1016/j.ocemod.2015.06.008.

Portilla-Yandún, J. and Cavaleri, L. (2016), On the specification of background errors for wave data assimilation systems. J. Geophys. Res. Oceans. Accepted Author Manuscript. doi:10.1002/2015JC011309

Portilla-Yardun, J., Ocampo-Torres, F.J., Monbaliu, J., 2009. Spectral partitioning and identification of wind sea and swell, Journal of Atmospheric and Oceanic Technology, Vol 26, 107-122.

Prandle, D., Wyatt, L.R., 1999. Editorial Introduction to this special issue, Coastal Engineering 37, 193-199.

Qi, J.C. Chen, R.C. Beardsley, W. Perrie and G. Cowles, 2009, An unstructured-grid finite-volume surface wave model (FVCOM-SWAVE): implementation, validations and applications. Ocean Modelling, 28, 153-166.

Qiao, F., Y. Yuan, Y. Yang, Q. Zheng, C. Xia, and J. Ma, 2004: Wave-induced mixing in the upper ocean: Distribution and application to global ocean circulation model. Geophys. Res. Lett., 31, L11303, doi:10.1029/2004GL019824. 
random waves. Proceedings of the 16th Conference on Coastal Engineering,

Rascle, N., F.Ardhuin, and E. A. Terray, 2006: Drift and mixing under the ocean surface: A coherent one-dimensional description with application to unstratified conditions. J. Geophys. Res., 111, C03016, doi:10.1029/2005JC003004

Rasmussen, J.H., 1998. Deterministic and Stochastic Modelling of Surface Gravity Waves in Finite Depth. Ph.D. thesis Technical University of Denmark.

Ricchi, A., M.M.Miglietta, F.Barbariol, A.Bergamasco, D.Bonaldo, A.Benetazzo, F.M.Falcieri, A.Russo, M.Sclavo, and S.Carniel, 2017. Sensitivity of a Mediterranean tropical-like cyclone to different model configurations and coupling strategies, Atmosphere, 8 (5), 92, doi:

10.3390/atmos8050092

Ricchiuto, M., Csík, A., and Deconinck, A..Residual distribution for general time dependent conservation laws.J.Comput.Phys., 209(1):249-289, 2005.

Riley, R., Teng, C.-C., Bouchard, R., Dinoso, R., Mettlach, T., 2011. Enhancements to NDBC's digital directional wave module, , Proceedings of MTS/IEEE Oceans 2011 Conference,Kona, Hawaii, September 2011.

Ris, R.C., L.H.Holthuijsen and N.Booij, 1999. A third-generation wave model for coastal regions: 2. Verification, J. of Geoph. Res. Oceans, 104, C4, 7667-7681, DOI: 10.1029/1998JC900123

Rogers, E.W., P.A.Hwang, and D.W.Wang, 2003. Investigation of wave growth and decay in the SWAN model: three regional scale applications, J.Phys.Oceanogr., 33, 366-389.

Rogers, W.E., and M.D. Orzech, 2013. Implementation and testing of ice and mud source functions in WAVEWATCH III ${ }^{\circledR}$, NRL Memorandum Report, NRL/MR/7320-13-9462, 31 pp.

Rogers, W.E., Holland, K.T., 2009. A study of dissipation of wind-waves by mud at Cassino Beach, Brasil: prediction and inversion. Cont. Shelf Res., 29, 676-690.

Rogers, W.E., J.M. Kaihatu, H.A. H. Petit, N. Booij, and L.H. Holthuijsen, 2002, Diffusion reduction in a arbitrary scale third generation wind wave model, Ocean Engng., 29, 1357-1390.

Rogers, W.E., Kaihatu, J.M., Hsu, L., Jensen, R.E., Dykes, J.D., Holland, K.T., 2007. Forecasting and hindcasting waves with the SWAN model in the Southern California Bight. Coastal Eng.54, 115.

Röhrs, J, KH Christensen, LR Hole and G Broström (2012). Observation-based evaluation of surface wave effects on currents and trajectory forecasts, Ocean Dyn, 62(10-12), pp 1519-1533, doi:10.1007/s10236-012-0576-y

Roland A., Zanke U., Hsu, T.W., Ou, S.H., Liau J.M. , 2006 “Spectral wave modelling on unstructured grids with the WWM (Wind Wave Model)" - I: The deep water case; Third ChineseGerman Joint Symposium on Coastal and Ocean Engineering (JOINT2006), Tainan, Taiwan

Roland, A. 2014: Application of Residual Distribution (RD) schemes to the geographical part of the Wave Action Equation, ECMWF Workshop on Ocean Waves, 25-27 June 2012, 
(https://www.ecmwf.int/sites/default/files/elibrary/2012/12002-application-residual-distribution-rdschemes-geographical-part-wave-action-equation.pdf).

Roland, A., 2008, Development of WWM II: Spectral wave modelling on unstructured meshes, PhD thesis, Inst. of Hydraul. and Water Resour. Eng., Tech. Univ. Darmstadt, Darmstadt, Germany.

Roland, A., A. Cucco, C. Ferrarin, T.-W.Hsu, J.-M.Liau, S.-H.Ou,G. Umgiesser, and U. Zanke 2009, On the development and verificationof a 2-D coupled wave-current model on unstructured meshes, J. Mar.Syst., 78, S244-S254.

Roland, A., Ardhuin, F., 2014. On the developments of spectral wave models: numerics and parameterisations for the coastal ocean. Ocean Dynamics, 64, 833-846.

Roland, A., Mewis, P., Zanke, U., Ou, S.H., Hsu, T.W. and Liau J.M., 2005. Verification and improvement of a spectral finite element wave model. Waves 2005, ASCE/COPRI, The Fifth International Symposium on Wave Measurement and Analysis, Ed. Billy Edge, Madrid, Spain.

Roland, A., Zhang, Y.J., Wang, H.V., Meng, Y., Teng, Y.C., Maderich, V., Brovchenko, I., DutourSikiric, M., Zanke, U., 2012. A fully coupled 3D wave-current interaction model on unstructured grids. J. Geophys. Res. 117:C00J33. doi:10.1029/2012JC007952

Romeiser, R., 1993. Global validation of the wave model WAM over a one-year period using Geosat wave height data, J. Geophy. Res. Vol 98, No. C3, 4713-4726.

Ruessink, B.G., Walstra, D.J.R., Southgate, H.N., 2003. Calibration and verification of a parametric wave model on barred beaches. Coast. Eng. 48, 139-149.

Rusu L., Guedes Soares C., 2015; Impact of assimilating altimeter data on wave predictions in the western Iberian coast, Ocean Modelling, V96, P1, 126-135, doi 10.1016/j.ocemod.2015.07.016.

Saetra, O, J Albretsen, P Janssen (2007). Sea-State-Dependent Momentum Fluxes for Ocean Modeling, J Phys Oceanogr, 37(11), pp 2714-2725, doi:10.1175/2007JPO3582.1

Salmon, J.E. 2016. Surf wave hydrodynamics in the coastal environment, $\mathrm{PhD}$ thesis Delft Univ. Tech., available at http://repositery.tudelft.nl, 155pp.

Salmon, J.E., Holthuijsen, L.H., 2015. Modelling depth-induced wave breaking over complex coastal bathymetries. Coastal Engineering, 105, 21-35.

Salmon, J.E., L. Holthuijsen, M. Zijlema, G. van Vledder, and J. Pietrzak. 2015. Scaling depthinduced wave breaking in two-dimensional spectral wave models. Ocean Model., 87, 30-47.

Salmon, J.E., Smit, P.B., Janssen, T.T., Holthuijsen, L.H., 2016. A consistent collinear triad approximation for operational wave models. Ocean Modelling. XX, DOI:

10.1016/j.ocemod.2016.06.009

Samiksha, S.V., 2015. Wave energy dissipation due to currents, suspended sediments and vegetation along the coast of India, PhD thesis, CSIR-National Institute of Oceanography, Dona Paula, Goa, India, 187pp.

Sanchez-Arcilla, A., M. Garcia-Leon, and V. Gracia. 2014. Hydro morphodynamic modelling in 
Mediterranean storms. Errors and uncertainties under sharp gradients. Nat. Hazard Earth Sys., 2(2), 1693-1728.

Scanlon, B, Ø Breivik, J-R Bidlot, P Janssen, A H Callaghan, B Ward (2016). Modelling whitecap fraction with a wave model, J Phys Oceanogr, doi:10.1175/JPO-D-15-0158.1

Sclavo M., A. Benetazzo, S. Carniel, A. Bergamasco, F.M. Falcieri, and D. Bonaldo. 2013. Wavecurrent interaction effect on sediment dispersal in a shallow semi-enclosed basin. In: Conley, D.C., G. Masselink, P.E. Russell, and T.J. O'Hare, T.J. (eds.), Proc., 12th International Coastal Symposium (Plymouth, England), J. Coast. Res., Special Issue, 65, 1587-1592.

Sclavo, M., L.Cavaleri, S.F.Barstow, and G.A.Athanassoulis, 2002. An efficient tool for wave climate analysis in European coastal waters, P.S.Z.N., Marine Ecology, 23, Supplement 1, 361-369.

Shchepetkin, A F and J C McWilliams (2005). The regional oceanic modeling system (ROMS): a split-explicit, free-surface, topography-following-coordinate oceanic model, Ocean Model, 9(4), pp 347-404, doi:10.1016/j.ocemod.2004.08.002

Sheldon, L and A Czaja (2014). Seasonal and interannual variability of an index of deep atmospheric convection over western boundary currents, Q J R Meteorol Soc, 140, pp 22-30, doi:10.1002/qj.2103

Shinu, R., V P Rao, S V Samiksha, P M Kessarkar, P Vethamony, SWA Naqvi, M T Babu, P K Dineshkumar, 2017, Suspended matter and fluid mud off Alleppey, southwest coast of India, Estuarine, Coastal and Shelf Science, 185, 31-43.

Siddons L., Wyatt L., Wolf J., 2009; Assimilation of HF radar data into the SWAN wave model, Journal of Marine Systems, V77, I3, 312-324, doi 10.1016/j.jmarsys.2007.12.017.

Skamarock, W.C., J.B. Klemp, J. Dudhia, D.O. Gill, D.M. Barker, W. Wang, and J.G. Powers. 2005. A description of the advanced research WRF Version 2. NCAR Technical Note, NCAR/TN468+STR.

Skyllingstad, E. D., and D. W. Denbo (1995), An ocean large-eddy simulation of Langmuir circulations and convection in the surface mixed layer, J. Geophys. Res., 100(C5), 8501-8522, doi:10.1029/94JC03202.

Smedman, A., X.G. Larsen, and U. Hogstrom, (2003). Effect of sea state on the momentum exchange over the sea during neutral conditions, J. Geophy. Res., 108(C11) 3367

Smit, P.B., 2014. Deterministic and Stochastic Modelling of Ocean Surface Waves. Ph.D. thesis Delft University of Technology. The Netherlands.

Smit, P.B., and T.T.Janssen, 2013.The evolution of inhomogeneous wave statistics through a variable medium, J. of Physic.Oceanogr.,43, 1741-1758.

Smit, P.B., T.T. Janssen, L.H. Holthuijsen, and J.M. Smith. 2014. Non-hydrostatic modeling of surf zone wave dynamics. Coast. Eng., 83, 36-48.

Smit, P.B., T.T.Janssen, and T.Herbers, 2015. Stochastic modeling of coherent wave fields over variable depth, J. of Physic. Oceanogr.,45, 1139-1154. 
Smith, G.A., Babanin, A.V., Riedel, P., Young, I.R., Oliver, S., Hubbert, G., 2011. Introduction of a new friction routine into the SWAN model that evaluates roughness due to bedform and sediment size changes. Coastal Engineering, 58, 317-326.

Smith, J. M., H.E. Bermudez, and B.A. Ebersole. 2000. Modeling waves at Willapa Bay, Washington, Proc., 27th International Conference on Coastal Engineering, 826-839.

Smith, J.M., 2002. Wave pressure gauge analysis with current, J. Waterway Port Coast. Ocean Eng. ASCE 128:21-275.

Smith, W.H.F. and Sandwell D.T., 1997. Global Seafloor Topography from Satellite Altimetry and Ship Depth Soundings, Science 277: 1956-1962, http://www.sciencemag.org/content/vol277/issue5334/.

Smyth, W. D., E. D. Skyllingstad, G. B. Crawford, and H. Wijesekera (2002), Nonlocal fluxes and Stokes drift effects in the K-profile parameterization, Ocean Dyn., 52(3), 104-115, doi:10.1007/s10236-002-0012-9.

Sorensen, O., Kofoed-Hansen, H., Rugbjerk, M. and Sorensen, L.S., 2004. A third generation spectral-wave model using an unstructured finite volume technique. Proceedings of the 29th International Conference on Coastal Engineering, Lissabon, Portugal.

Sørensen, O.R., Schäffer, H.A., Sørensen, L.S., 2004. Boussinesq-type modelling using an unstructured finite element technique. Coastal Eng., 50, 181-198.

Soulsby, R.L. 1995. Bed shear-stresses due to combined waves and currents. In: Stive, M., J. Fredsøe, L. Hamm, R. Soulsby, C Teisson, and J. Winterwerp (Eds.), Advances in Coastal Morphodynamics. Delft Hydraulics, Delft, The Netherlands, pp. 420-423.

Sportisse, B., 2000. An Analysis of Operator Splitting Techniques in the Stiff Case, Journal ofComputational Physics, Volume 161, Issue 1, 10 June 2000, Pages 140-168

Staneva, J, V Alari, Ø Breivik, J-R Bidlot, K Mogensen (2017). Effects of wave-induced forcing on a circulation model of the North Sea, Ocean Dyn, 67, pp 81-101, doi:10.1007/s10236-016-1009-0

Staneva, J., K.Wahle, W.Koch, A.Behrens, L.Fenoglio-Marc, and E.V.Stanev. 2016. Coastal flooding: impact of waves on storm surge during extremes - a case study for the German Bight, Nat. Hazards Earth Syst. Sci., 16, 2373-2389, doi.org/10.5194/nhess-16-2373-2016.

Steele, D.J., S.R. Dorling, R. Von Glasow, and J. Bacon, (2013). Idealized WRF model sensitivity simulations o fsea breeze types and their effects on offshore windfields, Atmos. Chem. Phys, 13, 443461.

Steele, K.E., Teng, C.-C., Wang, D.W.C., 1992. Wave direction wave measurements using pitch-roll buoys, IEEE J. Oceanic Engineering, Vol. 19, No. 4, 349-372.

Steele, K.E., Wang, D.W., Earle, M.D., Michelena, E.D., Dagnall, R.J., 1998. Buoy pitch and roll computed using three angular rate sensors, Coastal Engineering 35, 123-139.

Steenburgh, W.J., Schultz, D.M., Colle, B.A., 1998. The structure and evolution of gap outflow over the Gulf of Tehuantepec, Mexico. Mon. Weather. Rev., 126, 2673-2691. 
Stockwell, R.G., W.G Large, and R.F. Milliff, (2004). Resonant inertial oscillations in moored buoy ocean surface winds, Tellus, Vol. 56(5), 536-547.

Stokes, G. G. (1847), On the theory of oscillatory waves, Trans. Cambridge Philos. Soc., 8, 441-455. Sullivan, P.P., and J.C. McWilliams. 2010. Dynamics of winds and currents coupled to surface waves. Ann. Rev. Fluid Mech., 42, 19-42.

Sun, J. D. Vandermark, L. Mahrt, D. Vickers, T. Crawford and C. Vogel, (2001). Momentum transfer of the coastal zone, J. Geophy. Res., 106(D12), 123437-12,448.

Sutherland, G., G. Reverdin, L. Marié, and B. Ward (2014), Mixed and mixing layer depths in the ocean surface boundary layer under conditions of diurnal stratification, Geophys. Res. Lett.,41, 8469-8476, doi:10.1002/2014GL061939.

Sutherland, J., D.J.R. Walstra, T.J. Chesher, L.C. van Rijn, and H.N. Southgate. 2004. Evaluation of coastal area modeling systems at an estuary mouth, Coast. Eng., 51, 119-142.

Suzuki, T., Zijlema, M., Burger, B.,. Meijer, M.C., Narayan, S., 2011. Wave dissipation by vegetation with layer schematisation in SWAN. Coastal Engineering, 59, 64-71.

Swail, V., Jensen, R.E., Lee, B., Turton, J., Thomas, J., Gulev, S., Yelland, M., Etala, P., Meldrum, D., Birkemeier, W., Burnett, W., Warren, G., 2009. Wave measurements, needs and developments for the next decade, Proceedings of OceanObs'09, Vol. 2 II-1-87, 999-1008.

Tamura, H., Y. Miyazawa, and L.-Y. Oey (2012), The Stokes drift and wave induced-mass flux in the North Pacific, J. Geophys. Res., 117, C08021, doi:10.1029/2012JC008113.

Teixeira, M., and S. Belcher (2002), On the distortion of turbulence by a progressive surface wave, J. Fluid Mech., 458, 229-267, doi:10.1017/S0022112002007838.

Teng, C.-C., Bouchard, R.H., 2005. Directional wave measured from data buoys using angular rate sensors and magnetometers,5th Ocean Wave Measurement and Analysis, ASCE, Madrid, Spain. Thompson, E.T., 1977. Wave climate at selected locations along U.S. coasts, Technical Report, TR77-1, USACE Coastal Engineering Research Center, Fort Belvoir, Va.

Thomson, J., 2012. Wave breaking dissipation observed with "SWIFT' drifters, J. Atmos. Oceanic Technol., 29, 1866-1882.

Timpe, G.L., and Van de Voorde, N., 1995. NOMAD buoys: an overview of forty years of use, OCEANS'95, MTS/IEEE, Challenges of Our Changing Global Environment, Vol. 1, 309-315, 9-12 October 1995, San Diego, California.

Toledo, Y., T.-W. Hsu, and A. Roland, 2012. Extended time-dependent mild-slope and wave-action equations for wave-bottom and wave-current interactions, Proc. R. Soc. London A, 468, 184-205, doi:10.1098/rspa. 2011.0377.

Tolman H.L., Banner M.L., Kaihatu J.M., 2013. The NOPP operational wave model improvement project, J. Ocean Modelling, 70, 2-10. http://dx.doi.org/10.1016/j.ocemod.2012.11.011

Tolman, H. L., 2003. Treatment of unresolved islands and ice in wind wave models, Ocean 
Modelling, 5, 219-231.

Tolman, H. L., B. Balasubramaniyan, L. D. Burroughs, D. V. Chalikov, Y. Y. Chao, H. S. Chen, and V. M. Gerald (2002), Development and implementation of wind-generated ocean surface wave models at NCEP, Weather Forecasting, 17(2), 311-333, doi:10.1175/1520-

0434(2002)017<0311:DAIOWG>2.0.CO;2

Tolman, H.L. 1991. A third-generation model for wind waves on slowly varying, unsteady, and inhomogeneous depths and currents. J. Phys. Oceanogr., 21, 782-797.

Tolman, H.L., 1991. Effects of tides and storm surges on North Sea wind waves. J. Phys. Oceanography, 21, 766-781.

Tolman, H.L., 1992, Effects of numerics on the physics in a third-generation wind-wave model. J. Phys. Oceanogr., 22, 1095-1111.

Tolman, H.L., 1992. An evaluation of expressions for wave energy dissipation due to bottom friction in the presence of currents. Coastal Engineering, 16, 165-179.

Tolman, H.L., 2002a. Validation of WAVEWATCH III version 1.15 for a global domain.NOAA / NWS / NCEP / OMB Technical Note Nr. 213, 33 p.

Tolman, H.L., 2002b, Limiters in third-generation wind wave models, The Global Atmosphere and Ocean System, 8, 67-83.

Tolman, H.L., 2007. The 2007 Release of WAVEWATCH.10 Hindcasting and Forecasting, North Shore, Oahu, Hawaii.International Workshop on Wave.

Tolman, H.L., 2009. User manual and system documentation of WAVEWATCH-III version 3.14.NOAA / NWS / NCEP / MMAB Technical Note 222, $220 \mathrm{p}$

Trainor, L.T., 2009. Field observation and SWAN model Predictions of wave evolution in a muddy coastal environment. M.Sc. dissertation, Naval Post Graduate School, Monterey, 73pp.

Tsujimoto, K. and T. Koike, (2013). Land-lake breezes at low latitudes: The case of Tonle Sap Lake in Cambodia, J. Geophy. Res: Atmospheres, Vol. 118, 6970-6980.

Tucker, M.J., 1991. Waves in ocean engineering: Measurement, analysis, interpretation, Ellis Horwood, 431pp.

Tuomi, L., K.K. Kahma, and H. Pettersson, 2011. Wave hindcast statistics in the seasonally icecovered Baltic Sea, Boreal Env. Res., 16, 451-472.

Tuomi, L., Kahma, K., Fortelius, C., 2012. Modelling fetch-limited wave growth from an irregular shoreline. Journal of Marine Systems, 105-108, 96-105.

Uchiyama, Y., J.C. McWilliams, and A.F. Shchepetkin. 2010. Wave-current interaction in an oceanic circulation model with a vortex-force formalism: Application to the surf zone. Ocean Model., 34(1-2), 16-35.

Van der Westhuysen, A.J., A.A.Taylor, R.Padilla-Hernandez, A.Gibbs, P.Santos, D.Gaer, H.D.Cobb 
III, J.R.Lewitsky, and J.R.Rhome. Enhancements to the nearshore wave prediction system to provide coastal and overland hurricane wave guidance. Proc. 94th AMS Annual Meeting, Am. Meteor. Soc., Atlanta, 2014.

Van der Westhuysen, A.J., R.Padilla-Hernandez, P.Santos, A.Gibbs, D.Gaer, T.Nicolini, S.Tjaden, E.M.Devaliere and H.L.Tolman. Development and validation of the Nearshore Wave Prediction System. Proc. 93rd AMS Annual Meeting, Am. Meteor. Soc., Austin, 2013.

van Unen, R. F., vandBeuzekom, A.A., Forristall, G.Z., Mathisen,J.-P., Starke, J., 1998. WacsisWave crest sensor intercomparisonstudy at the MeetpostNoordwijk measurement platform. Oceans '98, Nice, France, IEEE, 192-197.

Veeramony J., Walker D., Hsu L., 2010; A variational data assimilation system for nearshore applications of SWAN, Ocean Modelling, V35, I3, 206-214, doi 10.1016/j.ocemod.2010.07.008.

Vignudelli, S., Scozzari, A., Woodworth, P., Wolf, J., Barbosa, S., Gomez-Enri, J., 2012. Development of radar altimetry data processing in the oceanic coastal zone, ESA/ESRIN Contract No. 21201/08/I-LG-CCN 3 (Phase 2), Report on validation of reprocessed height and waves, COASTALT2-EWP5-D52-v12b.doc, (http://www.coastalt.eu/files/results/COASTALT2-D5212b.pdf ).

Volume 227, Issue 7, 20 March 2008, Pages 3572-3594 Predicting weather, climate and extreme events..

Voorrips, A. C., V. K. Makin and S. Hasselmann, 1997; Assimilation of wave spectra from pitchand-roll buoys in a North Sea wave model, Journal of Geophysical Research, 102(C3), 5829-5849, 1997. doi:10.1029/96JC03242.

Wadhams, P., 1973. Attenuation of swell by sea ice, J. Geoph. Res., 78, 18, 3552-3563. Wahle K., Staneva J., Guenther H., 2015; Data assimilation of ocean wind waves using Neural Networks. A case study for the German Bight, Ocean Modelling, V96, P1, 117-125, doi 10.1016/j.ocemod.2015.07.007.

Walker, S. (1998). A Sailor's Wind, W.W. Norton \& Company, New York, NY, 362pp. Walsh, E.J., Wright, C.W., Vandemark, D., Krabill, W.B., Garcia, A.W., Houston, S.H., Murillo, S.T., Powell, M.D., Black, P.G., Marks Jr., F.D., 2002. Hurricane directional wave spectrum spatial variation at landfall. J. Phys. Oceanogr., 32, 1667-1684.

WAMDI, 1988. The WAM Model - A third Generation Ocean Wave Prediction Model. J. Phys. Oceanogr., 18, 1775-1810.

Wang, D.W., A.K. Liu, C.Y. Peng, and E.A. Meindl, (1994). Wave-current interaction near the Gulf Stream during the Surface Wave Dynamics Experiment, J. Geophy. Res, 99(C3), 5065-5079.

Ward, Brian, Tim Fristedt, Adrian H. Callaghan, Graig Sutherland, Xavier Sanchez, JérômeVialard, and Anneke ten Doeschate, 2014: The Air-Sea Interaction Profiler (ASIP): An Autonomous Upwardly Rising Profiler for Microstructure Measurements in the Upper Ocean. J. Atmos. Oceanic Tech 31.10 (2014): 2246-2267.

Warner, J C, B Armstrong, R He, J B Zambon (2010). Development of a Coupled OceanAtmosphere-Wave-Sediment Transport (COAWST) Modeling System, Ocean Model, 35(3), pp 230- 
Warner, J.C., N. Perlin, and E.D. Skyllingstad. 2008. Using the Model Coupling Toolkit to couple earth system models. Environ. Modell. Softw., 23, 1240-1249.

Warner, J.C., Sherwood, C.R., Signell, R.P., Harris, C., Arango, H.G., 2008. Development of a threedimensional, regional, coupled wave, current, and sediment-transport model. Computers and Geosciences, 34, 1284-1306.

Waters, J., Wyatt, L., Wolf, J., Adrian, H., 2013, Data assimilation of partitioned HF radar wave data into Wavewatch III. Ocean Modelling, 72. 17-31. 10.1016/j.ocemod.2013.07.003

Weber, J E (1983). Steady Wind- and Wave-Induced Currents in the Open Ocean, J Phys Oceanogr, 13, pp 524-530, doi:10/djz6md

Weller, R.A., Donelan, M.A., Brisco, M.G., and Huang, N.E., 1991. Riding the crest: A tale of two wave experiments," AMS Bulletin, 72, No. 2., 163-183.

Westerink, J.J., R.A. Luettich, J.C. Feyen, J.H. Atkinson, , C. Dawson, H.J. Roberts, M.D. Powell, J.P. Dunion, E.J. Kubatko, H. Pourtaheri, "A Basin to Channel Scale Unstructured Grid Hurricane Storm Surge Model Applied to Southern Louisiana," Monthly Weather Review, 136, 3, 833-864, 2008.

Westhuysen, A.J. Van der, 2010. Modeling of depth-induced wave breaking under finite depth wave growth conditions. J. Geophs. Res, 115, C01008, doi:10.1029/2009JC005433.

Westhuysen, A.J. Van der, Van Dongeren, A.R. , Groeneweg, J. Van Vledder, G.Ph., Peters, H., Gautier, C., Van Nieuwkoop, J.C.C., 2012. Improvements in spectral wave modelling in tidal inlets. J. Geophys. Res., 117, C00J28, doi:10.1029/2011JC007837.

Whitham, G.B., 1974.Linear and Nonlinear Waves, New York, John Wiley and Sons, 636 pp. Willebrand, J., 1975. Energy transport in a nonlinear and inhomogeneous random gravity wave field. J. Fluid Mech., 70, 113-126.

Willebrand, J., 1975. Energy transport in a nonlinear and inhomogeneous random gravity wave field. Journal of Fluid Mechanics 70, 113-126.

Winstead, N.S., Colle, B.A., and N. Bond, (2004). Synthetic aperture radar and high-resolution MM5 simulations of barrier jets in coastal Alaska, Geoscience and Remote Sensing Symposium, IGARAS'04, Proceedings IEEE International.

Winterwerp, J.C., de Graaff, R.F., Groeneweg, J., Luijendijk, A.P., 2007. Modelling wave damping at Guyana mud coast. Coastal Engineering, 54, 249-261.

Wolf, J., Brown J. M., Howarth M. J.: 2011, The wave climate of Liverpool Bay - observations and modelling. Ocean Dynamics, 61 (5). 639-665. 10.1007/s10236-011-0376-9

Wu, J., 1979: Oceanic whitecaps and sea state. J. Phys. Oceanogr., 9, 1064-1068, doi:10.1175/15200485(1979)0092.0.CO;2.

Wyatt, L.R., Green, J.J., Gurgel, K.-W., Nieto Borge, J.C., Reichert, K., Hessner, K., Gunther, H.,W. Rosenthal, W., Saetra, O., and Reistad, M., 2003. Validation and intercomparisons of wave measurements and models during the EuroROSE experiments, Coastal Engineering 48, 1-28. 
Wyatt, L.R., Green, J.J., MiddleditchA., 2009. Signal sampling inpacts on HF radar wave measurements, J. Atmos. Oceanic Technol., 26, 793-805.

Wyatt, L.R., Liakhovetsk, G., Graber, and H.C., Haus, B.K., 2005. Factors affecting the accuracy of SHOWEX HF radar wave measurements, J. Atmos. Oceanic Technol., 22, 847-859.

Yanenko, N.N., 1971, The method of fractional steps, Springer-Verlag.

Yildirim, B., Karniadakis. G-E., 2012, A hybrid spectral/DG method for solving the phase-averaged ocean wave equation: Algorithm and validation. J. Comput. Physics 231(14): 4921-4953.

Young, I.R. and L.A. Verhagen, (1996). The growth of fetch limited waves in water of finite depth. Part I: total energy and peak frequency, Coastal Engin. 28, 47-78.

Young, I.R. and Verhagen L.A. 1996b. The growth of fetch limited waves in water of finite depth. Part II: spectral evolution, Coastal Engineering 28, 79-100.

Young, I.R. and Verhagen, L.A. 1996a. The growth of fetch limited waves in water of finite depth. Part I: total energy and peak frequency, Coastal Engineering, 28, 47-78.

Young, I.R., 1999. Wind generated ocean waves. Elsevier, 288 pp.

Zieger, S., Babanin, A.V., Rogers, W.E., Young, I.R., 2015. Observation-based source terms in the third-generation wave model WAVEWATCH. Virtual Special Issue on Ocean Surface Waves, Ocean Modelling, 96, -2-25. http://dx.doi.org/10.1016/j.ocemod.2015.07.014.

Zijlema, M., 2009a, Parallel, unstructured mesh implementation for SWAN, in: J. M. Smith (Ed.), Proc. 31th Int. Conf. on Coast. Engng., Hamburg, Germany, 2009, 470-482.Zijlema, M., 2009b, Application of UnSWAN for wave hindcasting in the Dutch Wadden Sea, 11th Int. Workshop on Wave Hindcasting and Forecasting, Halifax, Canada, session M5.

Zijlema, M., 2009b, Multiscale simulations using unstructured mesh SWAN model for wave hindcasting in the Dutch Wadden Sea, in: M. Mizuguchi and S. Sato (Eds.), Proceedings of Coastal Dynamics 2009, World Scientific Publishing, Singapore, paper no. 2.

Zijlema, M., 2010, Computation of wind-wave spectra in coastal waters with SWAN on unstructured grids, Coastal Engineering, 57, 267-277.

Zijlema, M., and G.S. Stelling. 2008. Efficient computation of surf zone waves using the nonlinear shallow water equations with non-hydrostatic pressure. Coast. Eng., 55, 780-790.

Zijlema, M., Stelling, G.S., Smit, P.B., 2011. SWASH: An operational public domain code for simulating wave fields and rapidly varied flows in coastal waters. Coastal Engineering, 58, 992-1012.

Zijlema, M., Van Vledder, G.Ph., Holthuijsen, L.H., 2012. Bottom friction and wind drag for wave models. Coastal Engineering, 65, 19-26.

Zingone, E., and G. L. Hufford, (2006). Analyzing Surface Wind Fields near Lower Cook Inlet and Kodiak Waters Using SAR,14th Conference on Satellite Meteorology and Oceanography, Anchorage Alaska, Session J4-11, Anchorage, Alaska, NOAA/NWS, 30 Jan - 2 Feb. 2006. 
Zuh, M. and B.W. Atkinson, (2004). Observed and modelled climatology of the land-sea breeze 4407 circulation over the Persian Gulf, Internationa J. Climatol. 24: 883-905.

4408

4409 


\section{Appendix}

4411

4412 Acronyms

4413

4414 ADCP Acoustic Current Profiler

4415 CESM Community Earth System Model

4416 COAWST Coupled Ocean Atmosphere Wave Sediment Transport

4417 CTD Conductivity, Temperature, Depth

4418 DA Data Assimilation

4419 ECMWF European Centre for Medium-Range Weather Forecasts

4420 ECWAM ECMWF WAM

4421 ESA European Space Agency

4422 ISMAR Institute of Marine Sciences

4423 FNMOC Fleet Numerical Meteorology and Oceanography Center

4424 GLW Great Lakes Wave forecasting system

4425 GTS Global Telecommunication System

4426 IMLE Iterative Maximum LikElihood

4427 ITOP Impact of Typhoons on the Ocean in the Pacific

4428 JCOMM Joint technical Commission for Oceanography and Marine Meteorology

4429 JONSWAP JOint North Sea Wave Project

4430 KNMI Koninklijk Nederlands Meteorologisch Instituut

4431 LC Langmuir Circulation

4432 LTA lumped triad approximation

4433 MEM Maximum Entropy Method

4434 MLM Maximum Likelihood Method

4435 NCEP National Center for Environmental Prediction

4436 NDBC National Data Buoy Center

4437 NEMO Nucleus of European Modelling of the Ocean

4438 NOAA-NCEP National Ocean Atmospheric Administration - National Center for Environmental

4439 Prediction

4440 NWP Numerical Weather Prediction

4441 NWPS Nearshore Wave Prediction System

4442 OI Optimal Interpolation

4443 QA Quality Assurance 


$\begin{array}{lll}4444 & \text { QC } & \text { Quality Control } \\ 4445 & \text { rmse } & \text { root mean square error } \\ 4446 & \text { ROMS } & \text { Regional Ocean Model System } \\ 4447 & \text { SAR } & \text { Synthetic Aperture Radar } \\ 4448 & \text { SHOWEX } & \text { SHOaling Wave EXperiment } \\ 4449 & \text { SPB } & \text { stochastic parametric Boussinesq } \\ 4450 & \text { SWADE } & \text { Surface Wave Dynamics Experiment } \\ 4451 & \text { SWAN } & \text { Simulating WAves Nearshore } \\ 4452 & \text { TKE } & \text { Turbulent Kinetic Energy } \\ 4453 & \text { UKMO } & \text { U.K. Meteorological Office } \\ 4454 & \text { WAM } & \text { WAM wave model } \\ 4455 & \text { WFO } & \text { Weather Forecast Office } \\ 4456 & \text { WMO } & \text { World Meteorological Organisation } \\ 4457 & \text { WWIII, WW3 } & \text { WAVEWATCH III wave model } \\ 4458 & \text { WRF } & \text { Weather Research and Forecasting model } \\ 4459 & & \\ 4460 & & \end{array}$

